\title{
Aplicaciones de la tecnología de microondas a la desinsectación en los sectores: cárnico y de restauración de bienes culturales
}

Ángela Tatiana Zona Ortiz 


\title{
APLICACIONES DE LA TECNOLOGÍA DE MICROONDAS A LA DESINSECTACIÓN EN LOS SECTORES: CÁRNICO Y DE RESTAURACIÓN DE BIENES CULTURALES.
}
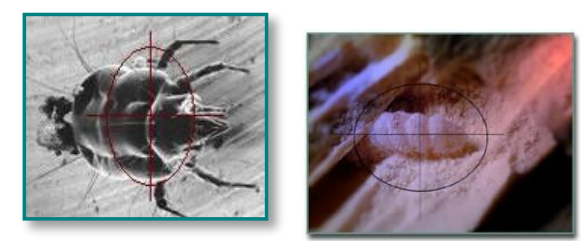

\section{TESIS DOCTORAL}

\author{
Doctorando: \\ Ing. Ángela Tatiana Zona Ortiz.
}

Directores:

Dr. Luis Nuño Fernández.

Dr. Juan Vicente Balbastre.

Departamento de Comunicaciones

Universidad Politécnica de Valencia

Valencia, 2007 
Esta editorial es miembro de la UNE, lo que garantiza la difusión y comercialización de sus publicaciones a nivel nacional e internacional.

\section{c Ángela Tatiana Zona Ortiz}

Primera edición, 2012

(c) de la presente edición:

Editorial Universitat Politècnica de València

www.editorial. upv.es

ISBN: 978-84-8363-905-4 (versión impresa)

Queda prohibida la reproducción, distribución, comercialización, transformación, y en general, cualquier otra forma de explotación, por cualquier procedimiento, de todo o parte de los contenidos de esta obra sin autorización expresa y por escrito de sus autores. 
A Dios.

A mi familia

Que a pesar de la distancia siempre ha estado a mi lado.

A mi esposo

Que siempre me brindó su apoyo en el largo proceso de ésta Tesis.

A mis tutores

Juan Vicente y Luis

Que me dieron conocimientos y herramientas para salir adelante con esta investigación. 


\section{RESUMEN}

Esta investigación amplia el campo de aplicación para el calor producido por las microondas en el área de desinsectación dentro de los sectores cárnico y de restauración de bienes culturales. Estableciendo un procedimiento inicial de aplicación que permita la conservación del material a desinsectar (jamón curado y madera), mientras se eliminan los agentes contaminantes (ácaros y carcoma). El objetivo que se persigue es la desinsectación segura, no invasiva y medioambientalmente limpia.

La metodología aplicada en ambos sectores consta de tres fases: establecimiento de los limites de temperatura admisibles por el material a desinsectar, determinación del tratamiento con mayor efectividad (mediante la aplicación de tratamientos admisibles sobre muestras compuestas por el material a desinsectar y el agente contaminante), y la verificación que el tratamiento elegido no produce efectos adversos sobre el material tratado.

En el caso del jamón curado se observó una gran efectividad a nivel de laboratorio, mientras que a nivel industrial se verificó la complejidad del problema por la interacción de diferentes factores. El alcance del caso llega a demostrar la viabilidad de la desinsectación con microondas.

En el caso de la madera se observó una alta mortalidad en el centro de la aplicación de microondas, mientras en las áreas periféricas es necesario el efecto calorífico acumulado de dos radiaciones. La profundidad del estudio llega hasta el establecimiento del tratamiento y procedimiento adecuados, además lleva al diseño de un nuevo aplicador de microondas que permita la independencia del tratamiento en relación con la orientación de las fibras de la madera esperando similares resultados.

En conclusión, la desinsectación del jamón curado mediante microondas es viable, se establece un tratamiento para tablas de madera con el prototipo disponible y se construye un nuevo prototipo que permite que el tratamiento sea independiente de la orientación de las fibras en la madera. 


\section{RESUM}

Aquesta investigació amplia el camp d'aplicació per al calor produït per les microones a l'àrea de desinsectació dins dels sectors càrnic i de restauració de béns culturals. Els resultats de la investigació permeten establir un procediment inicial d'aplicació que produeix la conservació del material a desinsectar (pernil curat i fusta), alhora que se'n eliminen els agents contaminants (àcars i corc de la fusta). L'objectiu que s'hi persegueix es la desinsectació segura, no invasiva i mediambientalment neta.

La metodologia aplicada a ambdós sectors consta de tres fases: establiment dels límits de temperatura admissibles pel material a desinsectar, determinació del tractament amb major efectivitat (mitjançant la aplicació de tractaments admissibles sobre mostres compostes pel material a tractar i l'agent contaminant), i la verificació de que el tractament escollit no produeix cap efecte negatiu sobre el material tractat..

En el cas del pernil curat s'observà una gran efectivitat a nivell de laboratori, però a nivell industrial es comprovà la complexitat del problema degut a la interacció de diferents factors. L'abast del cas no arriba a demostrar la viabilitat de la desinsectació amb microones.

En el caso de la fusta s'observà una altra mortalitat al centre de l'aplicació de microones, mentre que a les àrees perifèriques era necessari l'efecte calorífic acumulat de dues radiacions. La profunditat de l'estudi arriba fins a l'establiment del tractament i procediment adient: a més a més, porta al disseny d'un nou aplicador de microones que permeta la independència del tractament respecte de l'orientació de les fibres de la fusta obtenint resultats similars.

En conclusió, es comprova la viabilitat de la desinsectació del pernil curat mitjançant microones, s'estableix un tractament per a taulons de fusta amb un prototip dissenyat i fabricat com part de treball de la tesi i es construeix un nou prototip amb polarització circular que permet que el tractament siga independent de la orientació de les fibres de la fusta. 


\section{SUMMARY}

This research extends the application of microwave heating towards the disinfestation area specifically in two sectors: restoration of cultural heritage and meat industry. An initial procedure of application is established in both cases, these procedures preserve the material properties (cured ham and wood) while noxious agents are exterminated (mite or woodworm respectively). The main goal is the safe, non-invasive and environmentally friendly disinfestation.

The methodology in both cases has three different phases: the first one is to establish the temperature level that does not cause any damage on the material; the second one is to determine the more effective treatment by means of the application of different suitable treatments to a set of samples, which are composed of the material and noxious agents; the last one is to verify that the treatment does not cause any negative effect on the material.

In the case of the cured ham, an excellent effectiveness was observed in the laboratory, but at industrial level it was not possible due to the complexity of the problem. The goal was to demonstrate that the disinfestation of ham by microwaves is feasible.

In the case of wood, a high mortality was observed in the central point of the samples for a simple microwave application, while in the peripheral areas was necessary multiple microwave applications with an overlapped treatment. The goal was to establish the adequate treatment and procedure. Additionally, a new applicator prototype was designed in order to get an independent treatment to the fiber orientation of wood with similar results.

To summarize, the microwave disinfestations of cured ham is feasible, a suitable treatment to wood is established with the available prototype and a new prototype is built giving independence to fiber orientation. 


\section{TABLA DE CONTENIDO}

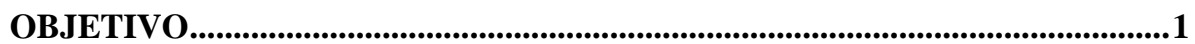

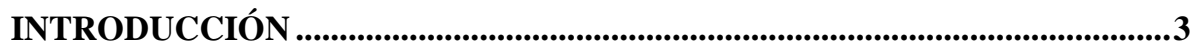

PRIMERA PARTE: TECNOLOGÍA DE MICROONDAS EN EL SECTOR

CÁRNICO .5

CAPÍTULO 1. PERMITIVIDAD DEL JAMÓN CURADO ...........................9

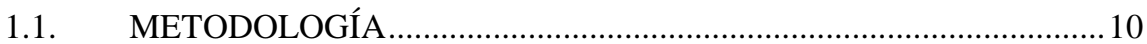

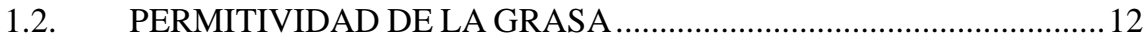

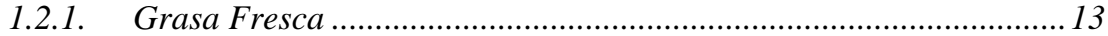

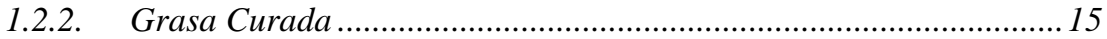

1.3. PERMITIVIDAD DEL MAGRO …................................................ 17

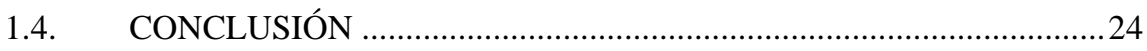

CAPÍTULO 2. DESINSECTACIÓN DE JAMÓN CURADO........................25

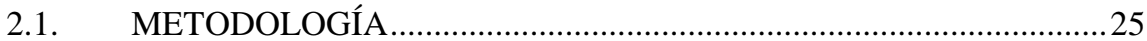

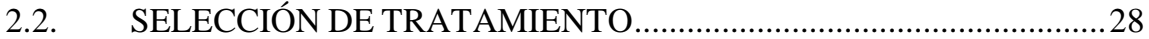

2.3. VERFICACIÓN DEL TRATAMIENTO …............................................ 31

2.3.1. Valoración sensorial ........................................................................ 32

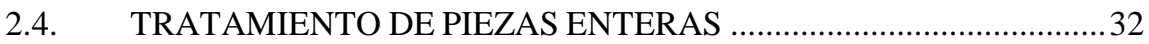

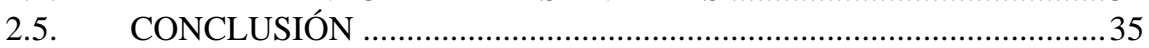

SEGUNDA PARTE: TECNOLOGÍA DE MICROONDAS EN EL SECTOR DE RESTAURACIÓN DE BIENES CULTURALES .......................................37

CAPÍTULO 3. DESINSECTACIÓN DE RETABLOS ..................................41

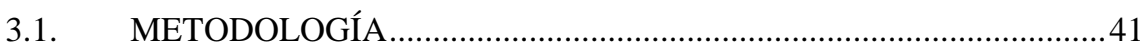

3.2. TIEMPO DE DURACIÓN DEL TRATAMIENTO …...........................49

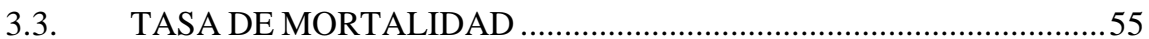

3.3.1. Modelos para radiaciones solapadas ...............................................56

3.3.2. Pruebas de temperatura para radiaciones solapadas .......................60 60

3.3.3. Tasa de Mortalidad con radiaciones solapadas ..............................6 62

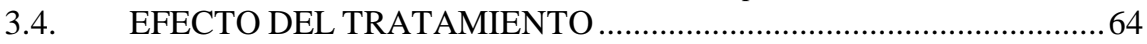

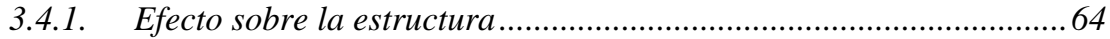

3.4.2. Efecto sobre la imprimación.............................................................6 65

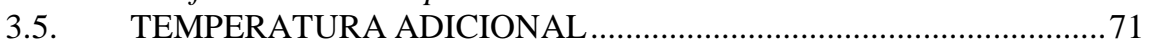

3.5.1. Temperatura en listones................................................................ 71

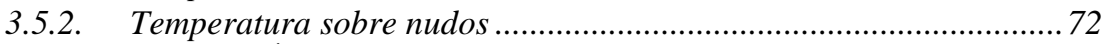

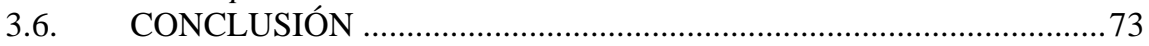

CAPÍTULO 4. DESARROLLO DE UN APLICADOR PARA

DESINSECTACION DE MADERA ................................................................................75 


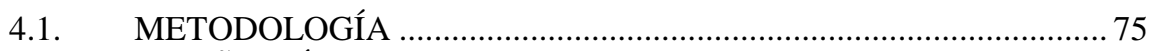

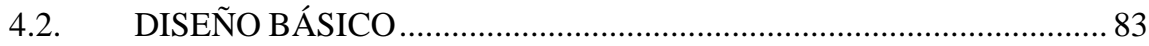

4.2.1. Modo de funcionamiento A .............................................................. 84

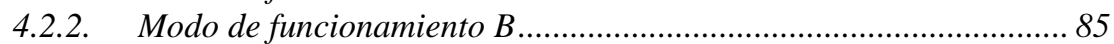

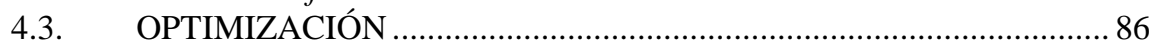

4.3.1. Longitud del conector interno ....................................................... 87

4.3.2. Ubicación de la alimentación ............................................................ 88

4.3.3. Ajustes para la construcción ......................................................... 93

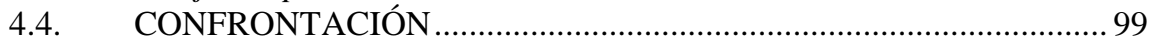

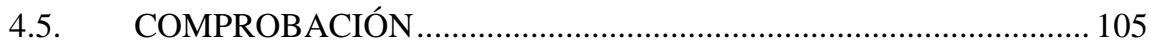

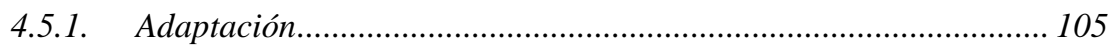

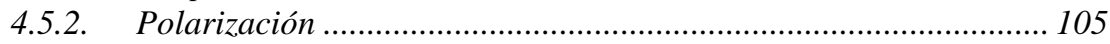

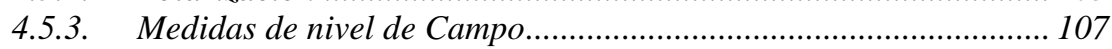

4.6. MEDIDAS DE TEMPERATURA ………………............................... 116

4.6.1. Temperatura en aplicación central................................................ 116

4.6.2. Temperatura en aplicación solapada …………………………..... 119

4.6.3. Efecto del calentamiento.......................................................... 122

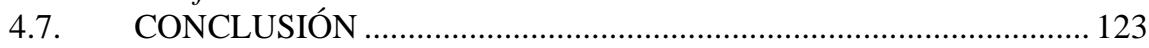

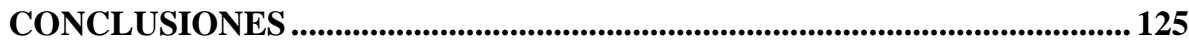

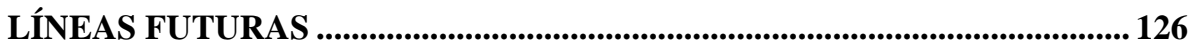

LOGROS CIENTÍFICOS.................................................................................. 127

REFERENCIAS ......................................................................................................... 128

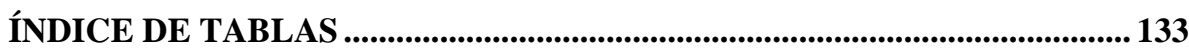

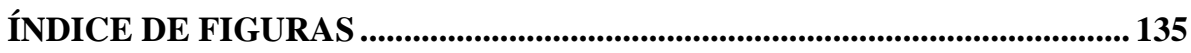

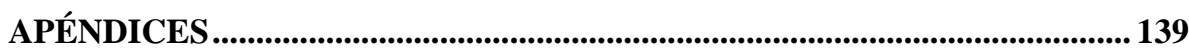

APÉNDICE I. PERMITIVIDAD DEL JAMÓN ……........................................ - 1 -

APÉNDICE II. DESISECTACIÓN DE RETABLOS........................................ - 3 -

APÉNDICE III. PLANOS ....................................................................... - 23 -

APÉNDICE IV. CAMPO ELÉCTRICO DEL DISEÑO BÁSICO ................. - 27 -

APÉNDICE V. COMPROBACIÓN APLICADOR ........................................ - 40 -

APÉNDICE VI. TEMPERATURA APLICADOR............................................ - 48 - 


\section{OBJETIVO}

Adaptar de manera experimental los conocimientos sobre la interacción de las microondas con los materiales y su efecto calorífico diferenciado, para aplicaciones específicas en el sector cárnico y en el sector de la restauración de bienes culturales.

Se forman grupos multidisciplinares y se aprovechan las potencialidades $\mathrm{y}$ equipamiento del Grupo de Electromagnetismo Aplicado (GEA) y sus colaboradores en los diferentes proyectos; con el fin de dar una solución no destructiva, higiénica, saludable y respetuosa con el medio ambiente a los problemas planteados.

La innovación radica fundamentalmente en ampliar el campo de acción de las microondas al sector cárnico y de restauración de bienes culturales en el contexto español y en la determinación del procedimiento inicial de aplicación. Esta investigación se realiza en el ámbito de la Universidad Politécnica de Valencia y su colaboración con otras entidades y empresas.

Durante la realización de esta tesis se quieren alcanzar los siguientes cuatro objetivos específicos:

1. Determinar si las propiedades dieléctricas del jamón permiten un tratamiento superficial del mismo mediante microondas, para salvaguardar el producto y eliminar los ácaros de la superficie.

2. Comprobar experimentalmente que el efecto calorífico alcanzado en los ácaros mediante la aplicación de microondas conduce a su muerte, manteniendo al mismo tiempo las propiedades organolépticas del jamón curado. Como resultado se obtiene una combinación potencia-tiempo para la desinsectación utilizando un horno de microondas.

3. Encontrar el tiempo y el procedimiento de aplicación de microondas que permita la exterminación de la carcoma en tablas de madera infestadas artificialmente, sin deteriorar la madera o la capa de imprimación donde se realizan las pinturas, utilizando un prototipo de antena con polarización lineal del grupo GEA.

4. Diseñar un aplicador para la desinsectación de madera y retablos con polarización circular y una cobertura de $10 \mathrm{~cm}$ de diámetro con facilidades para la aplicación de microondas in situ, además probar su funcionamiento y establecer las zonas de seguridad durante la aplicación de un tratamiento. 


\section{INTRODUCCIÓN}

La posibilidad de usar las microondas se hace patente a comienzos de los años cuarenta y posteriormente se introducen en los hogares en forma de horno microondas; hoy en día constituyen un término familiar en una gran variedad de ámbitos, y en un futuro seguramente serán ampliamente utilizadas en diversas aplicaciones y sectores de la industria. Estas ondas de radio comprenden el rango de frecuencias entre $300 \mathrm{MHz}$ y $300 \mathrm{GHz}$ dentro del espectro electromagnético, y su origen se remonta a la predicción matemática de Maxwell sobre la existencia y comportamiento de las ondas de radio (trabajando sobre las hipótesis de Faraday), luego dicha predicción fue verificada experimentalmente por Hertz.

La investigación central de esta tesis se basa en el principio de generación de calor con microondas, de lo cual la primera prueba fue aportada por d'Arsonval quien en 1895 descubrió que el efecto de las ondas de radio (entre $500 \mathrm{kHz}$ y $1,5 \mathrm{MHz}$ ) sobre los animales es la producción de calor [1].Sin embargo, la aplicación de las ondas de radio en el rango de microondas no fue posible hasta principio de los años cuarenta, cuando con el desarrollo del radar en la segunda guerra mundial se establece el magnetrón como generador de energía de microondas, desde entonces han sido ampliamente utilizadas en el sector de las comunicaciones como medio de transporte para la información.

No es hasta 1946 que se describe el calentamiento de radio frecuencia, éste se puede clasificar en calentamiento por macroondas o microondas. El primero incluye tanto el calentamiento inductivo como el capacitivo, mientras el segundo se refiere al calentamiento producido por los efectos de polarización a las frecuencias de microondas sobre un no conductor, generalmente éste último se lleva a cabo en una cavidad cerrada o un horno.

Es precisamente su característica de generación de calor la que permite utilizar las microondas en aplicaciones diferentes a las comunicaciones, estas aplicaciones abarcan sectores como la industria, la agricultura y la medicina. Algunos ejemplos son: la esterilización de suelos, la vulcanización del caucho, el secado de pieles, la desinsectación de arroz, la eliminación de las bacterias que producen las caries, entre otros. También se hace evidente el infinito campo para su aplicación en temas de secado, desinsectación, métodos no invasivos de cura de enfermedades o métodos no destructivos para medición de parámetros. Este tipo de energía tiene dos ventajas sobre los medios convencionales de calentamiento:

1. La energía de microondas constituye una fuente de calor volumétrica. El calor se genera en todo punto del volumen del material que se va a calentar, dependiendo de la amplitud del campo eléctrico en cada punto y de las propiedades dieléctricas de material procesado. 
2. La generación de calor a partir de la energía de microondas es selectiva, esto es, se genera más calor en aquellas zonas del material que son capaces de absorber más energía del campo eléctrico y transformarla en energía térmica. En particular, la generación de calor por microondas es significativamente alta en medios con alto contenido en agua.

Por estos dos puntos la energía de microondas es una alternativa muy interesante para la desinsectación en diferentes sectores, muestras de su eficacia en este campo se han demostrado en productos almacenados [2] [3] y frutos secos [4]. El trabajo de ésta tesis demuestra la viabilidad para la desinsectación mediante microondas tanto del jamón curado como de obras de arte con soporte de madera, también se establecen métodos y procedimientos orientativos de aplicación para cada caso.

Las frecuencias más utilizadas para este tipo de aplicaciones se encuentran en la denominada banda ISM (Industrial Scientific and Medical) comprendida entre 2,4 y 2,5 GHz, esta banda no necesita licencias y está reservada internacionalmente para uso no comercial de Radio frecuencias en los sectores industrial, científico y sanitario, adicionalmente el coste de los magnetrones en ésta frecuencia es reducido por su popularización gracias al desarrollo del tradicional horno microondas. Sin embargo existen otras frecuencias para uso en aplicaciones industriales, la siguiente comúnmente usada es $900 \mathrm{MHz}$ (869 MHz U.K. y $915 \mathrm{MHz}$ U.S.A.); y también están disponibles 433,9 MHz; 27,12 MHz ó 13,56 MHz.

El calentamiento con microondas es un proceso muy complejo y por lo tanto de difícil generalización, ya que debe tener en cuenta la interacción de las microondas tanto con el material a calentar como con el aplicador, y de estos dos entre sí[5]. El calentamiento del material depende tanto de las propiedades térmicas del mismo como de la distribución de la energía de microondas absorbida, la que a su vez depende de la forma del campo eléctrico del aplicador y de las propiedades dieléctricas del material. Dicho calentamiento se produce principalmente por la disipación de energía dentro del material, por lo tanto será mayor en medios con altas perdidas. La energía absorbida depende de varios factores como pueden ser: el volumen, el área, la forma, el tamaño, las propiedades (térmicas y dieléctricas), la presión, la densidad y el cambio de temperatura.

Por otro lado, la entrada de alimentación del aplicador es sumamente importante para obtener su patrón de campo y aprovechar al máximo la energía producida por el magnetrón. Conseguir la homogeneidad de campo no siempre es fácil y para reducir los costos del prototipo es necesario valerse de métodos numéricos tales como FDTD (Finite Difference Time Domain) o FEM (Finite Element Method) para modelar este fenómeno en 3D, generalmente el magnetrón se deja fuera de la modelación. Estos métodos son empleados en varios programas comerciales, para el desarrollo de esta tesis se utilizo HFSS de Ansoft Corporation.

La explicación física para la generación del calor mediante microondas es que las microondas entran en los materiales generando un efecto de polarización sobre las moléculas polares, éstas tratan de alinearse con el campo que está cambiando constantemente de orientación, entonces la energía electromagnética perdida en ese trabajo se transforma en el movimiento de dichas moléculas y este movimiento se manifiesta en forma de calor. 


\section{PRIMERA PARTE: Tecnología de microondas en el sector cárnico}

La industria de elaboración de jamón curado tiene una gran importancia en el sector cárnico español, tanto por su volumen de producción como por su importancia económica. Esta se debe no sólo al consumo interno sino también al externo, ya que ésta industria reporta grandes ganancias por la exportación de más del 17\% de la producción desde 1993, convirtiendo al jamón curado en uno de los productos más apreciados dentro del sector cárnico español.

El jamón curado es el producto resultante de una lenta y larga maduración de la carne en unas condiciones de temperatura, de concentración de sal y de secado perfectamente conjugadas, asegurando que no se presenten fenómenos de alteración microbiana.

El proceso de fabricación consta de dos fases claramente diferenciadas: una de estabilización o preservación de la materia prima y otra de maduración. La primera fase, como su nombre indica, tiene como fin lograr la estabilización del jamón, es decir, su preservación de los riesgos de alteración microbiana. Consta de dos etapas: salazón y post-salado, durante las cuales el jamón absorbe una determinada cantidad de sal que se distribuye en su interior, junto con las mermas que experimenta durante estas dos etapas se consigue una reducción de la actividad de agua hasta unos valores que inhiben el desarrollo de los microorganismos, que pueden causar alteraciones. La segunda fase de maduración transcurre durante un largo ciclo de secado donde tienen lugar complejos procesos enzimáticos que son los responsables del desarrollo de sus características sensoriales típicas.

Uno de los problemas que preocupa a la industria del jamón curado es la presencia de parásitos en el producto terminado. Este hecho origina importantes pérdidas económicas. El grupo de parásitos que reviste especial gravedad en cuanto a la calidad organoléptica y sanitaria del jamón curado es el de los artrópodos arácnidos de la familia de los Acáridos, y comúnmente los que más afectan a la industria del jamón son los ácaros del género Tyroglyphus, denominado “piojillo”. Estos parásitos se suelen desarrollar en la superficie de las piezas durante la fase de secado. Si las condiciones óptimas para su desarrollo se prolongan pueden incluso penetrar hacia el interior de las piezas, aunque normalmente se suelen desarrollar en las hendiduras y los cortes.

Estos parásitos (Ilustración 1) miden entre 0,2 y 0,5 mm y tienen un pequeño cuerpo traslucido. Su ciclo de vida varía entre 15 y 30 días dependiendo de la especie. Pueden sobrevivir en el rango de temperaturas de 18 a $45^{\circ} \mathrm{C}$ [6], aunque se desarrollan a temperaturas entre 25 y $30^{\circ} \mathrm{C}$ con una humedad relativa entre $60 \mathrm{y}$ $80 \%$. 


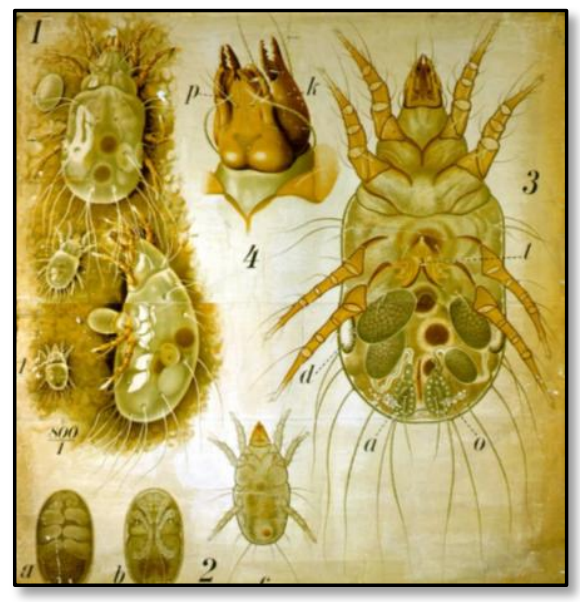

Ilustración 1

Los problemas más corrientes derivados de la presencia de ácaros son la formación de cuevas en el interior del jamón y la aparición de olores desagradables, además de la mala imagen que ocasionan ante el consumidor. En algunos casos, se ha relacionado la ingesta de ácaros con la aparición de eritemas, erupciones, conjuntivitis e incluso puede llegar a provocar una crisis asmática. Otro hecho importante es que en algunos países se prohíbe el consumo de jamones con ácaros.

Para luchar contra esta plaga se utilizan métodos preventivos y curativos, físicos, químicos, biológicos y bioquímicos. Sin embargo, no se cuenta con un método completamente eficaz para la erradicación de los ácaros del jamón curado.

Los métodos preventivos y curativos están basados en una esmerada higiene personal, la limpieza de secadores e instalaciones en general, el aislamiento y erradicación de focos contaminantes, etc. Una de las medidas más utilizadas es cubrir el jamón de manteca de cerdo antes y durante el secado; también son procesos utilizados con frecuencia en el saneamiento de piezas contaminadas, la inmersión de piezas en aceite, el cepillado y el flameado [7].

Sin embargo, todas estas acciones pueden no ser suficientes para controlar el crecimiento de ácaros en el jamón y se requiere entonces la ayuda de otros métodos que tengan una acción directa sobre el ácaro y frenen su desarrollo.

Entre los métodos físicos se destacan los tratamientos a altas y bajas temperaturas. Se ha demostrado que a $45^{\circ} \mathrm{C}$ los ácaros son eliminados en 1 hora pero el $70 \%$ de los huevos resisten el tratamiento, después de 5 h todos los estadios son eliminados; sin embargo el empleo de altas temperaturas puede causar graves daños en el producto al sufrir alteraciones en sus grasas[8], también se encuentra que a temperaturas inferiores a los $40{ }^{\circ} \mathrm{C}$ no se alcanza efecto acaricida. Por otro lado, en el empleo de aire caliente la temperatura debe ser superior a $145^{\circ} \mathrm{C}$ durante al menos 3 segundos[9]. 
En cuanto a bajas temperaturas se ha demostrado que una congelación rápida elimina los ácaros [9], de manera más exacta un tratamiento de $-28^{\circ} \mathrm{C}$ en periodos de 1 a 48 horas elimina diferente estadios pero en ningún caso los huevos[8].

Otro tipo de métodos físicos son las radiaciones ya sean de microondas, infrarrojos, ultravioletas, ionizantes o ultrasonidos. En relación con las microondas los estudios realizados parecen mostrar la ausencia de efecto acaricida. Sin embargo, es importante tener en cuenta los fenómenos electromagnéticos que intervienen en el proceso de calentamiento con microondas, ya que la distribución del campo es muy importante y más que la temperatura alcanzada por el jamón lo que se debe buscar es que el efecto diferenciado afecte en mayor medida a los ácaros.

En cuanto a la utilización de infrarrojos los resultados no son favorables, mientras que con los rayos ultravioleta se tienen resultados esperanzadores ya que después de 3 horas de radiación se tiene una mortalidad de 99\%; también se consigue una disminución del periodo de desarrollo, la fecundidad y el porcentaje de eclosión de los huevos. Por lo que se refiere a las radiaciones ionizantes, éstas están restringidas en productos alimenticios. Finalmente, los ultrasonidos sólo son efectivos en poblaciones reducidas, por lo que se recomienda su utilización como barrera física o tratamiento complementario [10].

Los plaguicidas industriales están prohibidos para su utilización sobre alimentos, de manera que su empleo queda restringido a la realización de un vacio sanitario con las instalaciones completamente vacías de alimentos [9], estos productos químicos no son siempre eficaces y además pueden conllevar riesgos para la salud.

Otro método químico es la utilización de atmosferas modificadas y empleo de gases, que en principio se presenta como una buena alternativa [11]. Sin embargo, la necesidad de instalaciones industriales costosas, complejas y específicas dificulta su aplicación. En el afán de encontrar productos naturales para el control de plagas se ha demostrado el efecto bactericida, bacteriostático y antifúngico de los aceites esenciales [9].

Otro procedimiento que ha sido estudiado para este fin es el control biológico mediante predadores ( $B$. dentriticus), que si bien parece ser efectivo en condiciones de laboratorio, fracasa a escala industrial debido a la elevada salinidad del jamón [10]. Por último se estudia el uso de feromonas de alarma [12], aunque su efectividad es cuestionada al entrar en contacto con las hormonas naturales de los ácaros [13]

En esta primera parte se pretende demostrar la viabilidad de la utilización de microondas en la desacarización de jamones a escala de laboratorio. Como primera medida se realizan medidas de sus propiedades dieléctricas con el fin de poder establecer cómo será la interacción de las microondas con el jamón. En cuanto a los ácaros, no se harán medidas de este tipo debido a su reducido tamaño. Es importante tener en cuenta que el jamón, al ser un material no homogéneo, anisótropo y no lineal presenta grandes complicaciones para hacer un planteamiento analítico.

En relación con los resultados negativos obtenidos en la bibliografía, cabe resaltar la importancia que tiene la homogeneidad de campo en la efectividad del tratamiento. Sin embargo, esta homogeneidad no se puede garantizar aunque se cuente en los 
hornos caseros con el plato giratorio y en los industriales con stirrer (ventilador con hélices metálicas que con su giro constante hace que las ondas reflejadas no sean siempre iguales logrando que el campo no sea estático dentro de la cavidad).

Adicionalmente, las zonas de campo eléctrico nulo dentro de la cavidad pueden servir de refugio para los ácaros durante el tratamiento, ya que en estas zonas no sufren los efectos de las microondas (más aun cuando los ácaros tienen un tamaño tan reducido y se mueven fácilmente).

El efecto de calentamiento de las microondas afecta en mayor medida a materiales compuestos en gran medida de agua. Debido a que los ácaros están formados mayoritariamente de agua, resulta inmediato pensar que esta tecnología podría ser una alternativa para la eliminación de los ácaros del jamón en su etapa de secado. Con microondas las altas temperaturas son alcanzadas más rápidamente por aquellos materiales con más perdidas, de esta manera la idea es que los ácaros alcancen una temperatura letal mientras el jamón se mantiene a temperaturas seguras para su buena conservación. 


\section{CAPÍTULO 1. PERMITIVIDAD DEL JAMÓN CURADO}

El método de las cavidades resonantes para la medida de propiedades dieléctricas [14] se basa en la reflexión y la teoría perturbacional, si la cavidad es excitada a la frecuencia apropiada, los campos pueden establecerse en la cavidad en ciertos modos de operación. En resonancia, las energías eléctrica y magnética medias serán iguales, y la energía total almacenada se irá intercambiando cíclicamente entre los campos eléctrico y magnético. Una cantidad relativamente pequeña de esta energía se pierde por la conductividad finita de las paredes y por el calentamiento dieléctrico sí hay un dieléctrico presente, por lo que los campos de la cavidad han de ser renovados a través de la fuente de excitación.

De este modo la introducción de una muestra de material dieléctrico en el interior de una cavidad provoca un descenso del valor tanto de la frecuencia de resonancia, como del factor de calidad. Así a partir de la medida de las frecuencias de resonancia y de los factores de calidad con y sin muestra en el interior de la cavidad, es posible el cálculo de la permitividad compleja del material que ha producido dicho cambio, sí se conocen las dimensiones de la cavidad y de la muestra.

Se considera una cavidad vacía no perturbada con frecuencia angular de resonancia $\omega_{0}$ y volumen $V$, en cada punto están definidos los campos $E_{0}$ y $H_{0}$. La permitividad eléctrica y la permeabilidad son respectivamente $\varepsilon_{0}$ y $\mu_{0}$. Al introducir un material de volumen $\Delta v$, caracterizado por $\varepsilon$ y $\mu$ en el interior de la cavidad, la frecuencia angular de resonancia se desplaza a $\omega$. Los campos en cada punto de la estructura formada se denotan por $E$ y $H$. Aplicando las ecuaciones de Maxwell sobre la cavidad y la muestra, se llega a la expresión:

$$
\frac{\omega-\omega_{0}}{\omega}=-\frac{\int_{\Delta v}\left[\left(\varepsilon-\varepsilon_{0}\right) E_{0}^{*} \cdot E+\left(\mu-\mu_{0}\right) H_{0}^{*} \cdot H\right] d v}{\int_{v}\left[\varepsilon_{0} E_{0}^{*} \cdot E+\mu_{0} H_{0}^{*} \cdot H\right] d v}
$$

La Ecuación da el cambio relativo en la frecuencia angular de resonancia producido por la inserción de la muestra de dieléctrico con volumen $\Delta v$, dentro de una cavidad resonante de volumen $V$ llena de aire. La integral en el numerador es sobre el volumen de la muestra, mientras que la del denominador es sobre el volumen de la cavidad. El signo negativo en la parte derecha de la ecuación indica que la frecuencia de resonancia disminuye al introducir la muestra de material dieléctrico. Cuando la muestra es pequeña comparada con la longitud de onda la teoría perturbacional permite una simplificación en los cálculos, además sí la muestra es introducida en un lugar de campo eléctrico máximo y campo magnético nulo la permeabilidad no afecta los cálculos. 


\subsection{METODOLOGÍA}

En este capítulo se quiere establecer la permitividad de los componentes del jamón fresco y curado, con el objetivo de determinar la profundidad alcanzada por un tratamiento con microondas. Para obtener la permitividad del magro y la grasa se usa el método perturbacional en una cavidad cilíndrica parcialmente rellena, método explicado al principio de este capítulo.

Se utiliza una cavidad resonante (Figura 1-1) que funciona en el modo $\mathrm{TM}_{010}$, el radio interno (c) es $6,15 \mathrm{~mm}$, el radio externo (a) es $49 \mathrm{~mm}$, la altura (h) es $20,2 \mathrm{~mm}$ y se alimenta mediante una sonda magnética. Las paredes interiores están recubiertas en plata para disminuir el factor de pérdidas de la cavidad y está diseñada conforme a las condiciones de la teoría perturbacional para la medida de propiedades dieléctricas [15]

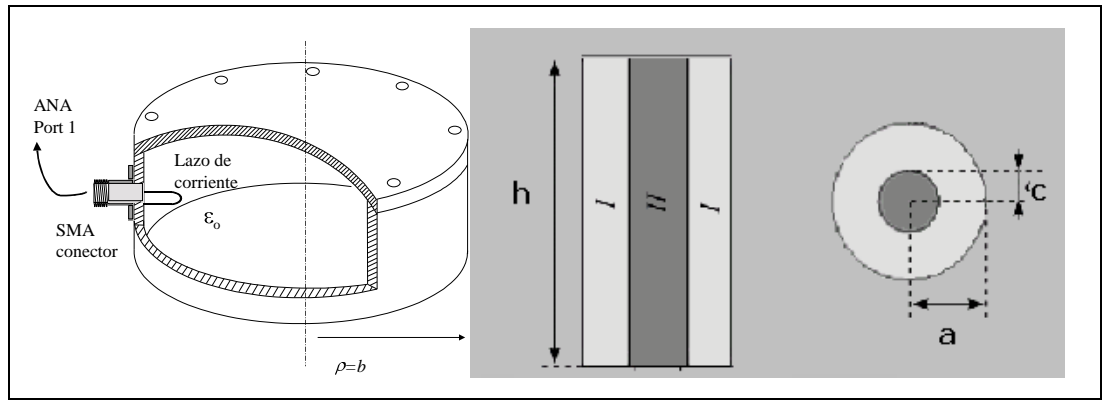

Figura 1-1. Cavidad Cilíndrica

Se sabe que los campos $\mathrm{Ez}$ y $\mathrm{H} \phi$ en una cavidad Cilíndrica $\mathrm{TM}_{010}$ son los únicos diferentes de cero:

$$
\begin{gathered}
E_{Z}=-j \frac{\beta r^{2}}{\omega r \mu \varepsilon} B_{010} J_{0}\left(\frac{X_{01}}{a} \rho\right) \\
H_{\varphi}=-\frac{X_{01}}{a} \frac{B_{010}}{\mu} J^{\prime}{ }_{0}\left(\frac{X_{01}}{a} \rho\right)
\end{gathered}
$$

El modo dominante en la cavidad depende de sus dimensiones, en este caso el modo domínate es $\mathrm{TM}_{010}$ mientras $\mathrm{h} / \mathrm{a}$ sea menor que 2,03. La frecuencia de resonancia está dada por:

$$
f r=\frac{1}{2 \pi \sqrt{\mu \varepsilon}} \sqrt{\left(\frac{X_{m n}}{a}\right)^{2}+\left(\frac{p \pi}{n}\right)^{2}}=\frac{1}{2 \pi \sqrt{\mu \varepsilon}} \sqrt{\left(\frac{X_{01}}{a}\right)^{2}}=2,34 \mathrm{GHz}
$$

Conociendo estas características, el cálculo de la permitividad se hace mediante un software desarrollado en Matlab [16] [17] (Figura 1-2), que calcula la constante dieléctrica y el factor de pérdidas a partir de la frecuencia de resonancia y el factor 
de calidad, estos datos son sacados de la medida del $\mathrm{S}_{11}$ hecha con un analizador de redes (HP8753ES ó HP8720).

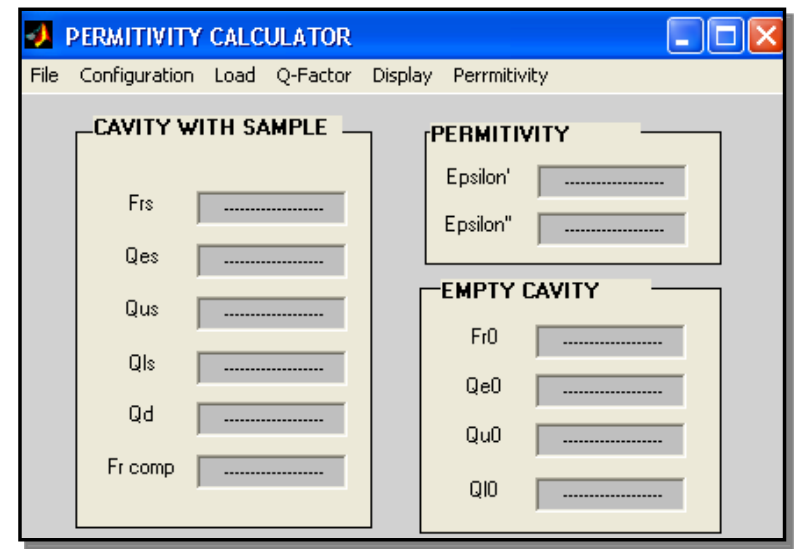

Figura 1-2. Software para el cálculo de la permitividad.

El procedimiento para obtención del las propiedades dieléctricas se muestra en la Figura 1-3. Primero se mide el coeficiente de reflexión de la cavidad vacía, luego se introduce la muestra en forma de barra cilíndrica en el centro de la cavidad rellenando toda la altura, se mide nuevamente el coeficiente de reflexión, y finalmente se introducen ambos datos en el software que calcula el valor de las propiedades dieléctricas.

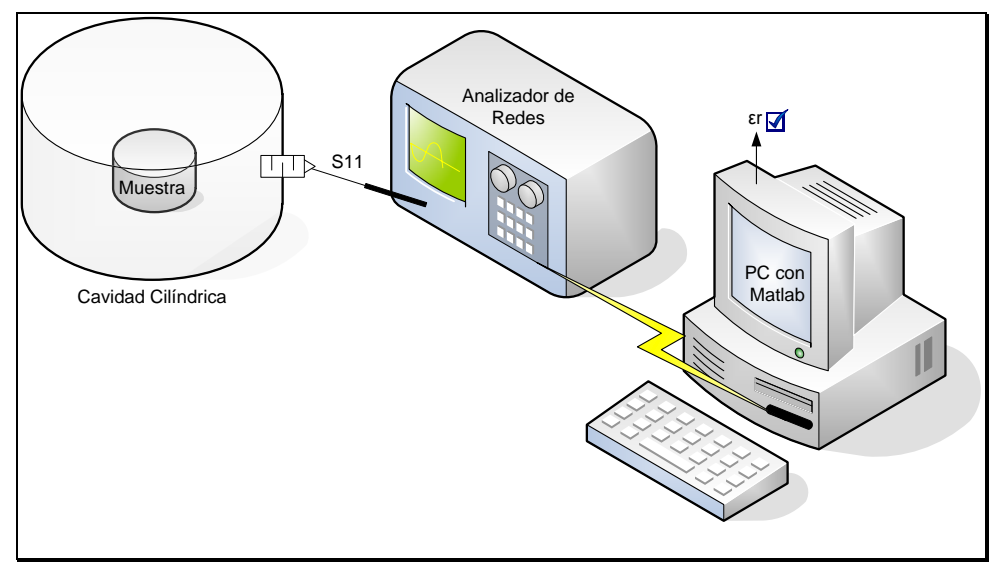

Figura 1-3. Configuración para la medida de permitividad.

En general, las muestras están contenidas en un tubo plástico de ensayo donde se colocan al ser extraídas de la pieza original con un saca-bocados. El diámetro exterior de dicho tubo es 11,67 $\mathrm{mm}$ con un grosor de 1,5 $\mathrm{mm}$ y su influencia sobre la medida es despreciable (Apéndice I. i). 


\subsection{PERMITIVIDAD DE LA GRASA}

Los valores de permitividad son calculados a partir de la frecuencia de resonancia y el factor de calidad con y sin muestra, por lo tanto es necesario confirmar que el origen de dichos valores no afecte notablemente los valores de permitividad obtenidos con el software para las diferentes muestras.

Por un lado, el software puede calcular la frecuencia y el factor de calidad a partir de las medidas de $\mathrm{S}_{11}$ o se pueden introducir directamente los valores visualizados en el analizador. La frecuencia es la misma ya sea calculada por el software o vista en el analizador, pero no es así para los factores de calidad; sin embargo el resultado de permitividad no varía de manera apreciable como puede verse en la Figura 1-4, de esta manera en los casos que no se puede medir el $\mathrm{Q}$ en el analizador, se puede calcular mediante el software asegurando resultados similares de permitividad.

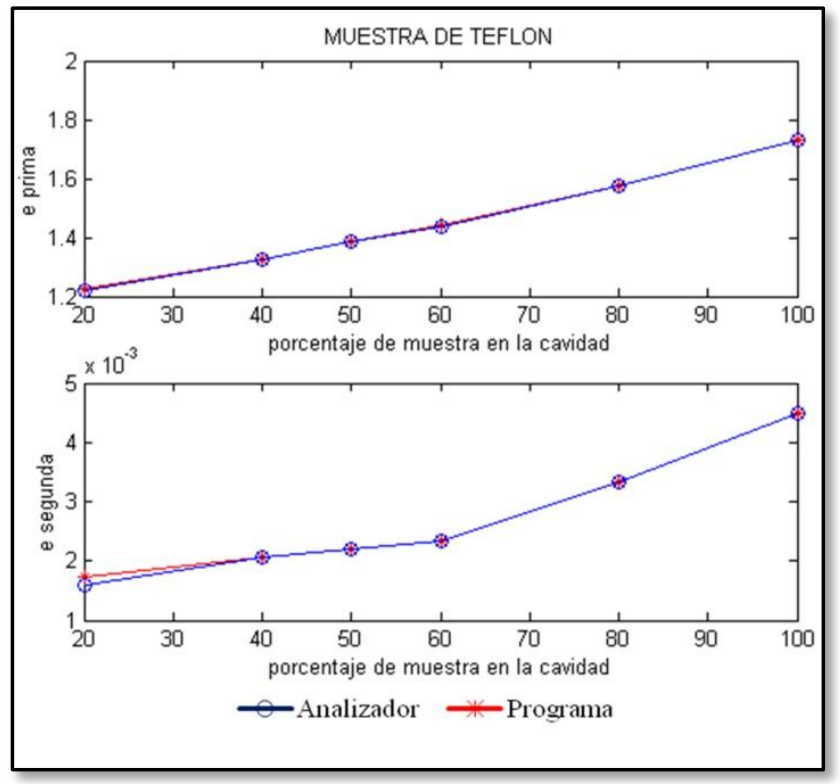

Figura 1-4. Permitividad - Frecuencia y $Q$ de origen diferente

Por otro lado, se dispone de dos tipos de analizadores para hacer las medidas de $\mathrm{S}_{11}$ : HP8753 y HP8720. En la Figura 1-5 se muestra la diferencia entre la medida obtenida por cada analizador, el rango de frecuencias está entre 2,1 y 2,4 GHz, la diferencia en relación con la frecuencia es despreciable pero la del Q es de $2 \%$ en el caso de la cavidad vacía y $1 \%$ para la cavidad con muestra, sin embargo esta pequeña diferencia no es significativa para el cálculo de la permitividad mediante el software. 


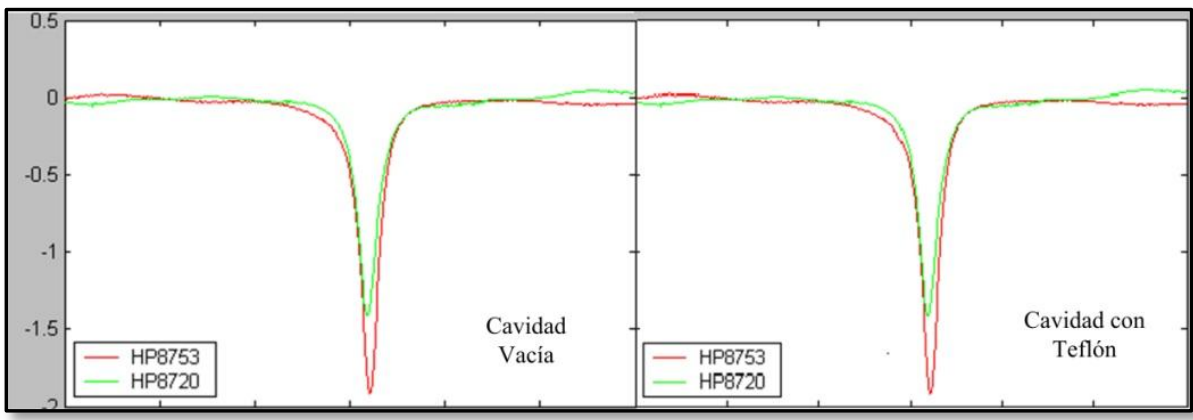

Figura 1-5. $S_{11}$ con diferentes analizadores

Partiendo del hecho que sin importar el origen de los datos de frecuencia y Q, los valores del permitividad son similares y por lo tanto comparables, se pasa al cálculo de permitividad para muestras de grasa.

\subsubsection{Grasa Fresca}

Se midieron 7 muestras en el rango de frecuencias entre 1,5 y 2,5 $\mathrm{GHz}$ para tres casos diferentes:

- Caso 1: Medidas realizadas con el analizador HP8720 (Figura 1-6) y alimentando la cavidad con la sonda magnética No. 5. Sólo se obtuvieron resultados para cinco de las siete muestras.

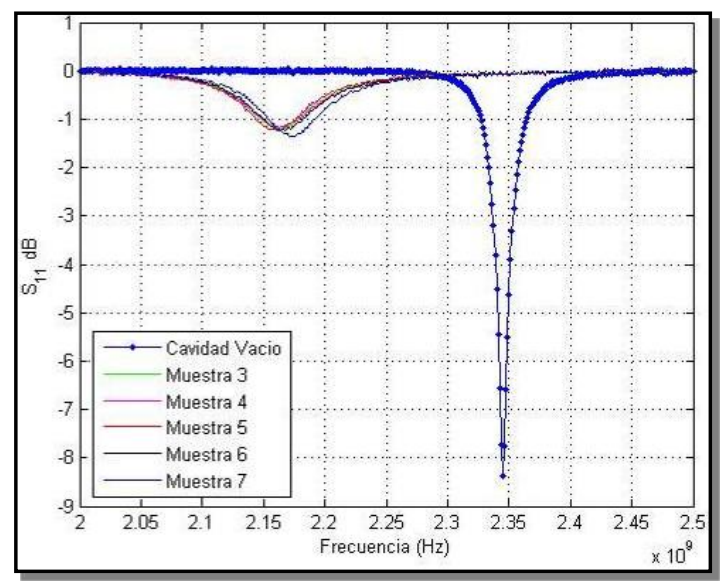

Figura 1-6. Resonancias caso 1

- Caso 2: Medidas realizadas con el analizador HP8720 (Figura 1-7) y alimentando la cavidad con la sonda No. 7. 


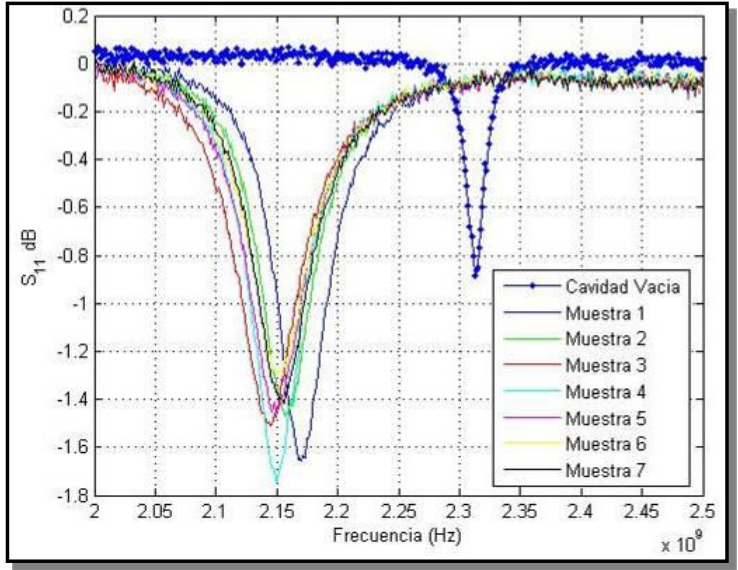

Figura 1-7. Resonancias caso 2

- Caso 3: Medidas realizadas con el analizador HP8753 (Figura 1-8) y alimentando la cavidad de nuevo con la sonda No. 7.

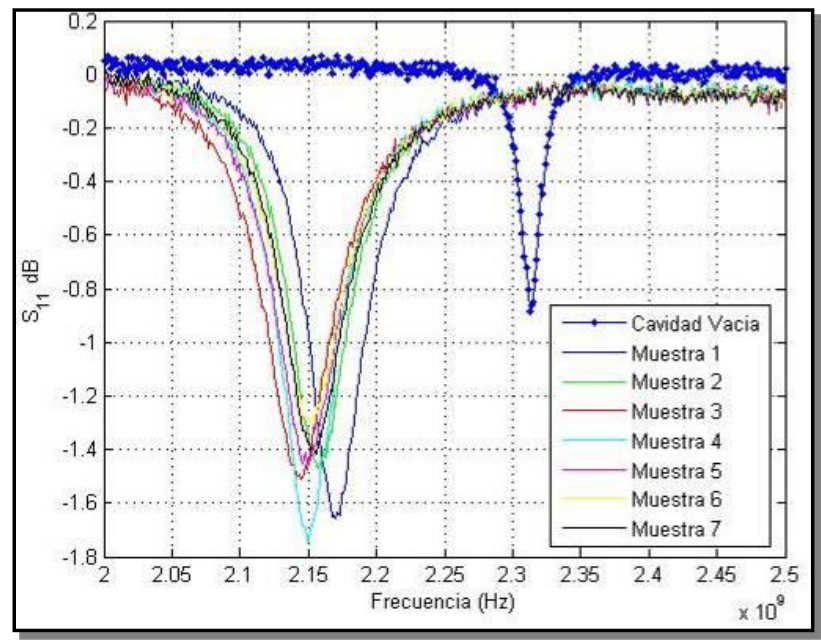

Figura 1-8. Resonancias caso 3

En la Figura 1-9 se muestran los resultados obtenidos (Datos Apéndice I. ii). Se observa que los valores de la constante dieléctrica (épsilon prima ó $\varepsilon$ ') son más constantes que los valores el factor de pérdidas (épsilon segunda ó ع") en los tres casos. En el caso 1 se observa un valor que está por encima del promedio para el factor de pérdidas, razón por la cual el caso 1 no es tenido en cuenta en el cálculo de la mediana de la Tabla 1-1. 


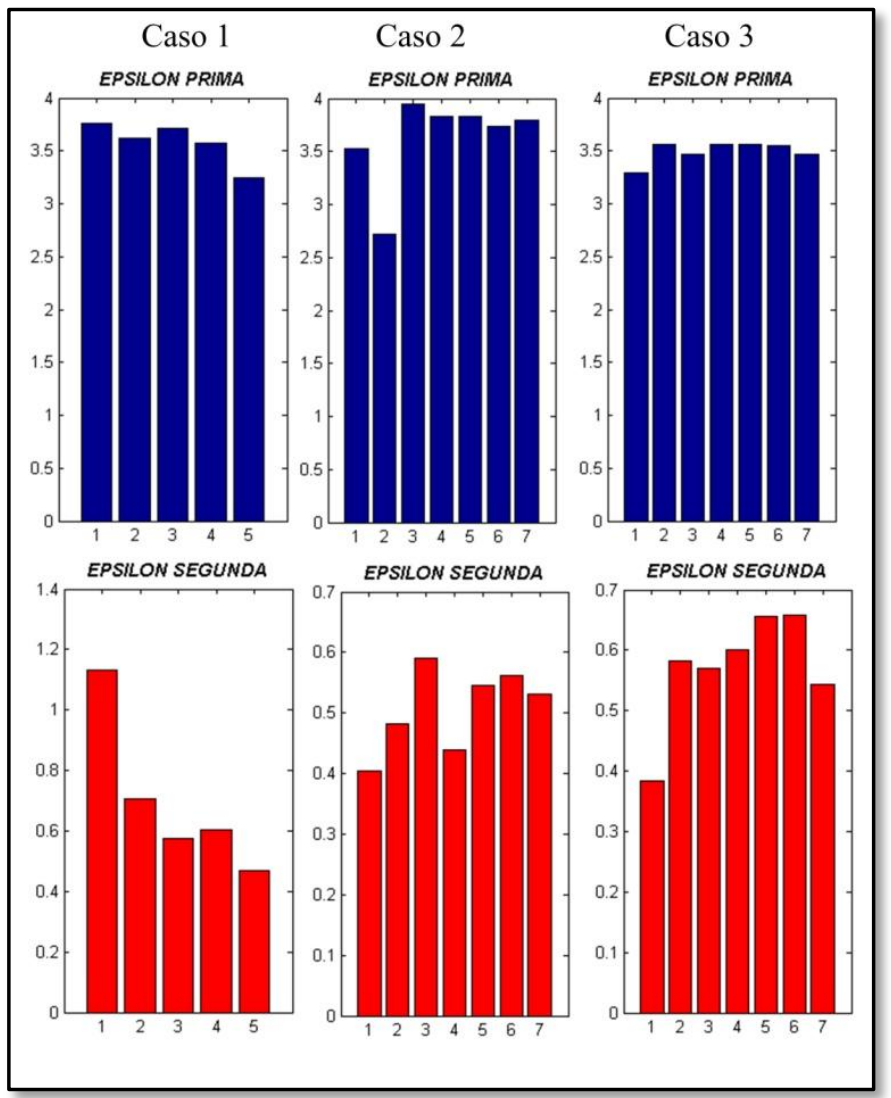

Figura 1-9. Medidas de permitividad - grasa fresca

Tabla 1-1. Permitividad de grasa fresca

\begin{tabular}{|l|c|c|c|}
\hline \hline & Mediana & $\begin{array}{c}\text { Desviación } \\
\text { Estándar }\end{array}$ & $\begin{array}{c}\text { Índice de } \\
\text { Dispersión }\end{array}$ \\
\hline $\boldsymbol{\varepsilon}^{\prime}$ & 3,5597 & 0,1905 & 0,0535 \\
\hline $\boldsymbol{\varepsilon}^{\prime}$ & 0,5538 & 0,0826 & 0,1491 \\
\hline
\end{tabular}

\subsubsection{Grasa Curada}

En el proceso de secado del jamón las capas de grasa se hacen más delgadas, por lo que obtener muestras de grasa curada se hace complicado. Para este caso solo se han medido tres muestras con el analizador HP8720 (Figura 1-10) y alimentando la cavidad con la sonda No. 7. El rango de frecuencias es de 2 a 2,6 GHz. Los resultados obtenidos ese muestran en la Figura 1-11 y el resumen de los mismos en la Tabla 1-2 (Apéndice I. iii). 


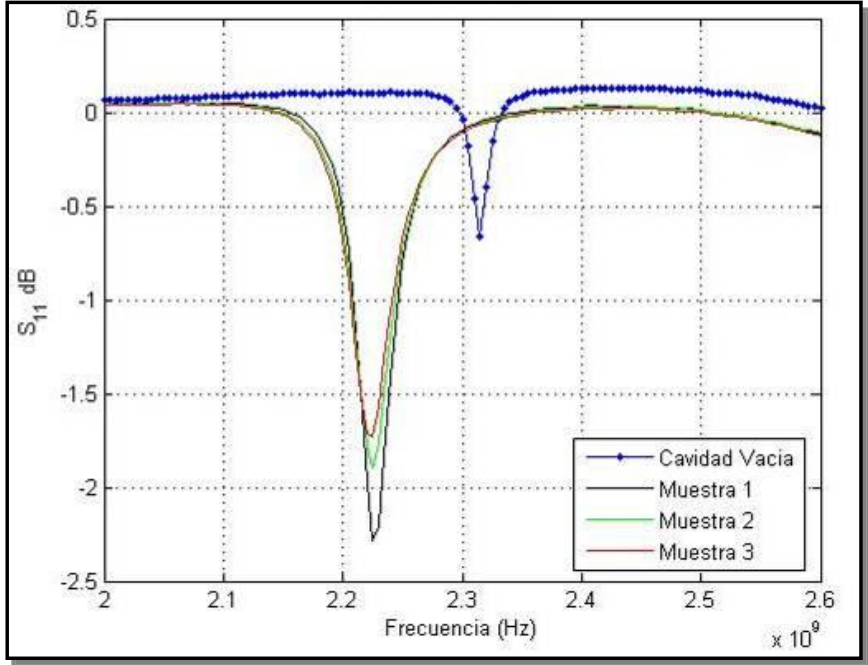

Figura 1-10. Resonancias grasa curada

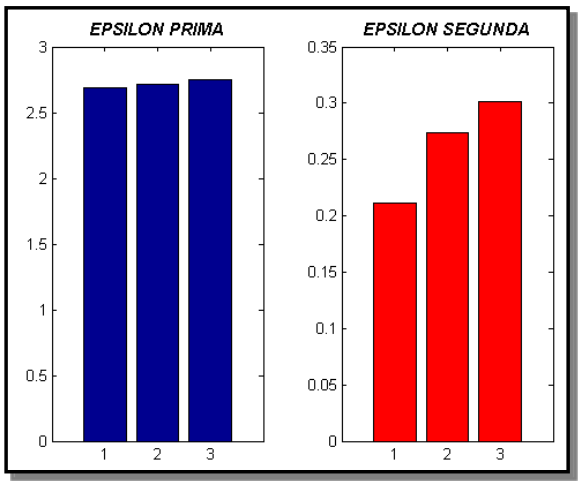

Figura 1-11. Medidas de permitividad-grasa curada

Tabla 1-2. Permitividad de grasa curada

\begin{tabular}{|c|c|c|c||}
\hline & Mediana & $\begin{array}{c}\text { Desviación } \\
\text { Estándar }\end{array}$ & $\begin{array}{c}\text { Índice de } \\
\text { Dispersión }\end{array}$ \\
\hline$\varepsilon^{\prime}$ & 2,7148 & 0,0255 & 0,0094 \\
\hline$\varepsilon "$ & 0,2735 & 0,0394 & 0,1440 \\
\hline
\end{tabular}

En la literatura [5] están medidos: el aceite de girasol con 2,4 de constante dieléctrica, 0,18 de factor de pérdida y una profundidad de penetración de $20,3 \mathrm{~cm}$; y la grasa con una constante dieléctrica de 2,5 , un factor de pérdidas de 0,15 y una profundidad de penetración 17,07. 


\subsection{PERMITIVIDAD DEL MAGRO}

Al seguir el procedimiento anterior con el magro la perturbación producida por la muestra no se puede observar claramente, por lo que la sonda No. 7 se cambia por una de mayor longitud (No. 8); de este modo se obtiene la perturbación producida por una muestra de magro fresco introducida totalmente en la cavidad (Figura 1-12), la frecuencia de resonancia de la cavidad con la muestra de magro es $1,8079 \mathrm{GHz}$.

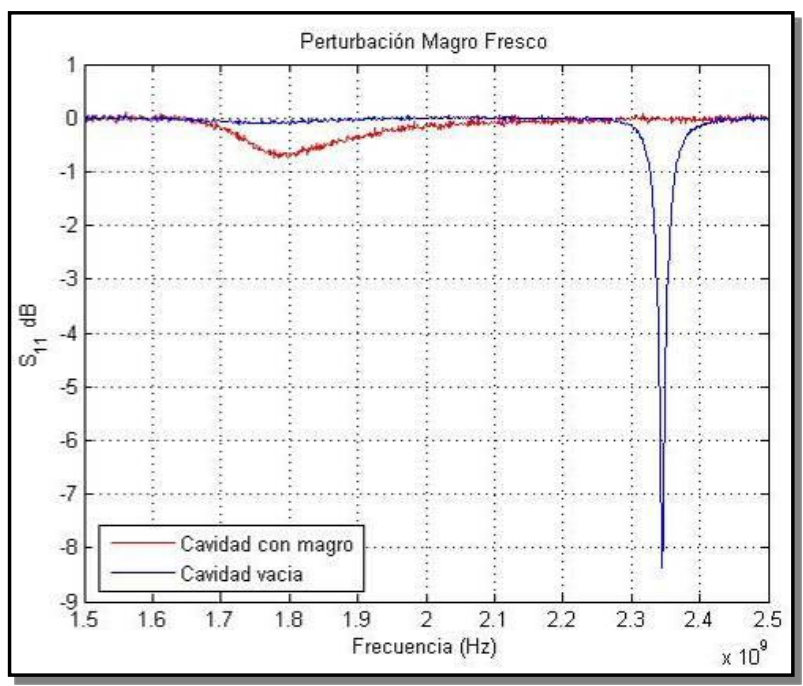

Figura 1-12. Perturbación magro fresco

El valor de la permitividad calculado a partir de dicha medida es 9,5937 para la constante dieléctrica ( $\left.\varepsilon^{\prime}\right)$ y de 1,8747 para el factor de pérdidas ( $\left.\varepsilon^{\prime \prime}\right)$. En el caso de magro curado no se puede observar la perturbación para ninguna de las muestras introducidas totalmente.

Teniendo en cuenta que las propiedades dieléctricas del jamón cocido a 2,8 GHz y a $20{ }^{\circ} \mathrm{C}$ son 43 para la constante dieléctrica y 22,5 en el caso del factor de pérdidas; del jamón precocinado son 42,9 para la constate dieléctrica y 22,8 para el factor de pérdidas y del jamón fresco son 53,2 y 15,7 manteniendo el orden anterior [5], es necesario revisar el método.

Se hace una simulación en la que se parametrizan tanto la constante dieléctrica (1,5100) como el factor de pérdidas $(0,5-10)$, para una muestra introducida al $100 \%$ (Figura 1-13). Los resultados revelan un salto de modo con una frecuencia de resonancia de 1,8 $\mathrm{GHz}$ para una constante dieléctrica de 45,27 y un factor de pérdidas superior a 2,66; lo que indica que la medida realizada no es correcta y las propiedades verdaderas correspondientes a dicha muestra se acercan a los valores encontrados en la literatura. 


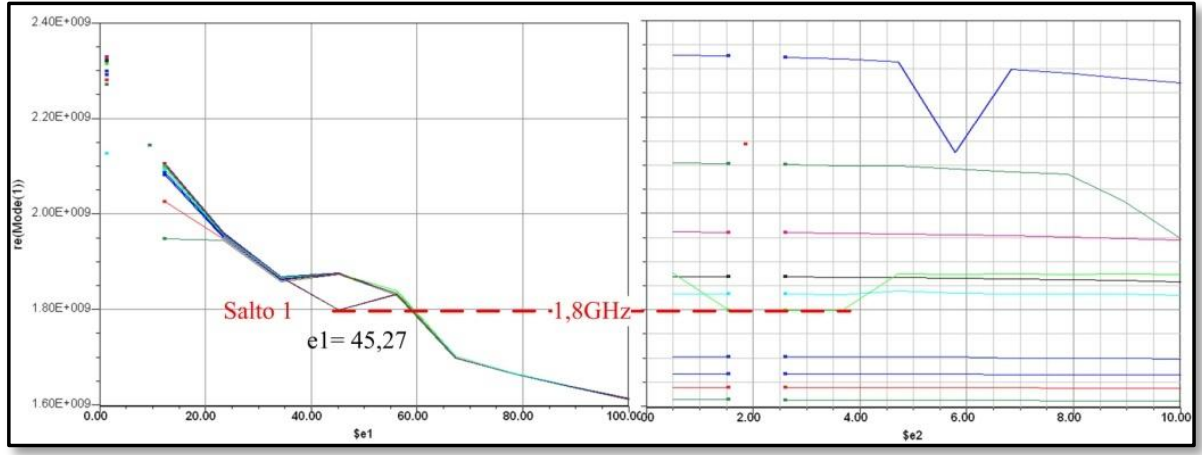

Figura 1-13. Frecuencia a 100\% de profundidad

Por otro lado, para asegurar que la onda en el dieléctrico sea evanescente y la perturbación pequeña [18] debe asegurarse que:

$$
\frac{b^{2}}{m^{2}} \gg a^{2} \varepsilon
$$

Suponiendo mucho mayor como 10 veces más grande, con un radio de cavidad $(b)$ de $49 \mathrm{~mm}, m$ igual a 1 y un radio de la muestra $(a)$ de $6,15 \mathrm{~mm}$, la constante dieléctrica medible con esta cavidad tendrá que ser menor o igual a 6,348, confirmando de esta manera la imposibilidad de usar este método para la medida del magro, si se desea medir una permitividad mayor será necesario utilizar cavidades con un radio mucho mayor.

En cuanto al efecto de los hoyos de inserción dependen de si se comportan como una guía al corte o no, estos efectos son despreciables para longitudes mayores a $5 \mathrm{~mm}$ [19], de $7 \mathrm{~mm}$ para el PTFE y $2 \mathrm{~mm}$ para el agua [20]. En la cavidad disponible esta longitud es de $40 \mathrm{~mm}$, por lo que el error de las medidas no se debe a los hoyos de inserción, sino exclusivamente al salto de modo que se produce por la gran perturbación que causa la muestra de magro.

En resumen, es necesario cambiar de método para estimar las propiedades dieléctricas del magro debido a que son muy altas y no se puede utilizar un método perturbacional con el software y la cavidad disponibles.

El método a utilizar es el de la caracterización dieléctrica de materiales a partir de medidas de transmisión y reflexión en líneas de transmisión. La idea es usar los parámetros de dispersión $S_{11}$ y $S_{21}$ para calcular la permitividad y permeabilidad compleja de un material, que rellena la sección interior de una guía de onda o cable coaxial (método Nicolson-Ross)[21].

El problema plantea una línea de transmisión formada por tres medios: un fragmento de material dieléctrico de longitud $d$, que rellena por completo el interior de una guía de onda y dos accesos abiertos en los extremos. Con este planteamiento se pueden calcular los parámetros $\mathbf{T}$, a partir de los cuales se obtienen los parámetros $\mathbf{S}$. 
Definiendo dos parámetros adicionales, que contienen toda la información necesaria para el cálculo de la permitividad y permeabilidad compleja, tales que:

$$
\begin{gathered}
\Gamma=\frac{Z_{1}-Z_{0}}{Z_{1}+Z_{0}} \\
\mathrm{~T}=\mathrm{e}^{-\gamma \cdot \mathrm{d}}
\end{gathered}
$$

Se pueden expresar los parámetros $\mathbf{S}$ en función de dichos parámetros adicionales con las siguientes expresiones:

$$
\begin{aligned}
& S_{11}(\omega)=S_{22}(\omega)=\frac{\Gamma\left(1-\mathrm{T}^{2}\right)}{1-\Gamma^{2} \mathrm{~T}^{2}} \\
& S_{12}(\omega)=S_{21}(\omega)=\frac{\mathrm{T}\left(1-\Gamma^{2}\right)}{1-\Gamma^{2} \mathrm{~T}^{2}}
\end{aligned}
$$

Haciendo la relación inversa se tiene que:

$$
\begin{gathered}
\Gamma=K \pm \sqrt{K^{2}-1} \\
\mathrm{~T}=\frac{\left(S_{11}(\omega)+S_{21}(\omega)\right)-\Gamma}{1-\left[S_{11}(\omega)+S_{21}(\omega)\right] \cdot \Gamma}
\end{gathered}
$$

Siendo el valor de K de la forma:

$$
K=\frac{\left(S_{11}^{2}(\omega)-S_{21}^{2}(\omega)\right)+1}{2 \cdot S_{11}(\omega)}
$$

Los parámetros de dispersión se obtienen de la medida directa mediante un analizador de redes, suponiendo la unión entre los dos medios (dieléctrico-aire) como suficientemente lisa, para medios lineales y homogéneos, se puede considerar que el modo a propagar es el fundamental $\mathrm{TE}_{01}$, de esta manera la permitividad y permeabilidad complejas se pueden expresar como:

$$
\begin{gathered}
\mu_{r}=\frac{1+\Gamma}{\Lambda \cdot(1-\Gamma) \cdot \sqrt{\frac{1}{\lambda_{0}^{2}}-\frac{1}{\lambda_{c}^{2}}}} \\
\varepsilon_{r}=\frac{\lambda_{0}^{2}\left(\frac{1}{\Lambda^{2}}+\frac{1}{\lambda_{c}^{2}}\right)}{\mu_{r}}
\end{gathered}
$$


Donde:

$$
\frac{1}{\Lambda^{2}}=-\left[\frac{1}{2 \pi \cdot d} \ln \left(\frac{1}{\mathrm{~T}}\right)\right]^{2}
$$

En ésta última expresión hay que obtener el logaritmo neperiano de un número complejo que presenta una indeterminación en fase,

$$
\ln \left(\frac{1}{\mathrm{~T}}\right)=\ln \left|\frac{1}{\mathrm{~T}}\right|+j\left(\operatorname{fase}\left(\frac{1}{\mathrm{~T}}\right)+2 \pi \cdot n\right)
$$

Donde $n$ representa el entero de la relación $\left(d / \lambda_{\mathrm{g}}\right)$. Además, el cálculo de $\Gamma$ presenta una indeterminación de signo que se soluciona imponiendo $|\Gamma| \leq 1$. Este método tiene un aspecto negativo para materiales con bajas pérdidas, ya que presenta divergencias a frecuencias correspondientes a $\lambda_{\mathrm{g}} / 2$.

El método Barker-Jarvis (de máxima precisión) resuelve las divergencias del método Nicolson-Ross a costa de un procesado más lento, ya que tiene que iterar una función para calcular la permitividad, esta función es:

$$
\frac{1}{2}\left(\left[S_{12}+S_{21}\right]+\Phi \cdot\left[S_{11}+S_{22}\right]\right)=\frac{\mathrm{T} \cdot\left(1-\Gamma^{2}\right)+\Phi \cdot \Gamma \cdot\left(1-\mathrm{T}^{2}\right)}{1-\mathrm{T}^{2} \cdot \Gamma^{2}}
$$

En ésta se hace referencia a los planos de la muestra no a los de calibración. El valor $\Phi$ es una constante que varía en función de la longitud de la muestra, la incertidumbre de los parámetros de scattering y las pérdidas dieléctricas estimadas del material. Como regla general, $\Phi$ puede venir dado por el cociente entre la incertidumbre en $S_{21}$ y la incertidumbre en $S_{11}$. Se parte de la definición de los parámetros $\Gamma \mathrm{y} \mathrm{T}$ como:

$$
\begin{gathered}
\Gamma=\frac{\frac{\gamma_{0}}{\mu_{0}}-\frac{\gamma}{\mu}}{\frac{\gamma_{0}}{\mu_{0}}+\frac{\gamma}{\mu}} \\
\mathrm{T}=e^{-\gamma \cdot L}
\end{gathered}
$$

Para suplir las carencias de los anteriores métodos, se desarrolla un método no iterativo, que partir de las mismas definiciones del método Nicolson-Ross añade un paso intermedio para introducir los parámetros electromagnéticos efectivos definidos como:

$$
\begin{gathered}
\mu_{e f f}^{*}=\frac{\lambda_{0 g}}{\Lambda}\left(\frac{1+\Gamma}{1-\Gamma}\right) \\
\varepsilon_{e f f}^{*}=\frac{\lambda_{0 g}}{\Lambda}\left(\frac{1-\Gamma}{1+\Gamma}\right)
\end{gathered}
$$


Estos parámetros presuponen un modo de propagación TEM, teniendo en cuenta que las impedancias y constante de propagación están definidas como:

$$
\begin{gathered}
\gamma=\gamma_{0} \sqrt{\mu_{e f f}^{*} \varepsilon_{e f f}^{*}} \\
Z=Z_{0} \sqrt{\frac{\mu_{e f f}^{*}}{\varepsilon_{e f f}^{*}}}
\end{gathered}
$$

La permitividad y permeabilidad del material están dadas por:

$$
\begin{gathered}
\mu_{r}=\mu_{e f f}^{*} \\
\varepsilon_{r}=\left(1-\frac{\lambda_{0}^{2}}{\lambda_{c}^{2}}\right) \varepsilon_{e f f}^{*}+\frac{\lambda_{0}^{2}}{\lambda_{c}^{2}} \frac{1}{\mu_{e f f}^{*}}
\end{gathered}
$$

Para materiales dieléctricos $\left(\mu^{*}=\mu^{*}\right.$ eff $\left.=1\right)$ es posible establecer una nueva expresión de la permitividad compleja efectiva (método de permitividad rápido):

$$
\varepsilon_{e f f}^{*}=\varepsilon_{e f f}^{*} \mu_{e f f}^{*}=\frac{\lambda_{0 g}^{2}}{\Lambda^{2}}
$$

Para concluir con el objetivo de este apartado se utilizan guías de onda WR340 cuyas dimensiones son 86x43 mm, un analizador de redes y el programas Agilent Technologies $85071 \mathrm{~V}$ D1.00, que incorpora los modelos de medida de reflexión y transmisión anteriormente explicados:

1. Mu y Épsilon, con el modelo de Nicolson-Ross.

2. Épsilon con precisión, sólo para materiales no magnéticos.

3. Épsilon rápido, sólo para materiales no magnéticos.

La Figura 1-14 muestra la configuración de las medidas. La calibración se realiza en el rango de frecuencias comprendido entre 2 y $3 \mathrm{GHz}$ para 201 puntos, el plano calibración del puerto 1 es el de la guía que se encuentra a la izquierda en la figura y el del puerto 2 se encuentra al finalizar el holder. Una vez terminada la calibración full two port se estable en el programa la longitud del holder como 0, la frecuencia para medidas en guía como $1,73 \mathrm{GHz}$ y se escoge el método de cálculo de la permitividad. 


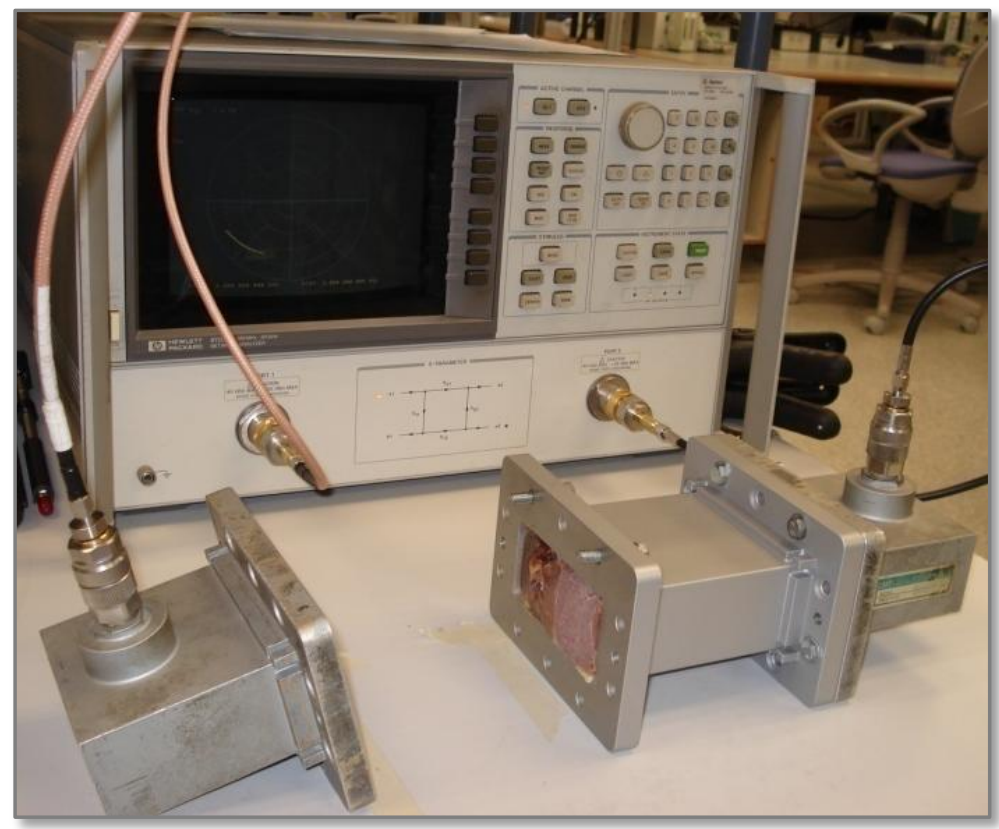

Figura 1-14. Configuración de medida de permitividad en guía.

En la Figura 1-15 se observan las cinco muestras utilizadas. Al utilizar este método es importante que la superficie de las muestras sean lo más lisa posible y cada muestra se ajuste a las dimensiones de la cavidad. El grosor de las muestras es 7,5 $\mathrm{mm}$, la distancia comprendida entre el puerto y la muestra es $0 \mathrm{~mm}$, esto puede ser así ya que la calibración se hace con el holder como parte del puerto 2.

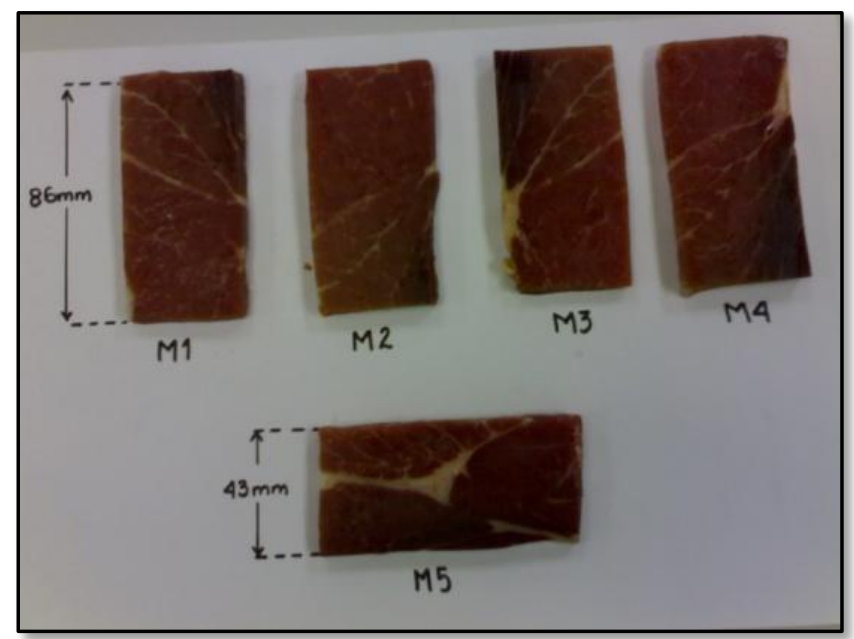

Figura 1-15. Muestras para medidas en guía

Los resultados obtenidos para la frecuencia de $2,45 \mathrm{GHz}$ se muestran en la Tabla 1-3 
Tabla 1-3. Medidas de permitividad en guía

\begin{tabular}{|c|c|c|c|l||}
\hline MUESTRA & PESO $(\mathbf{g})$ & $\boldsymbol{\varepsilon}$ & $\boldsymbol{\varepsilon} ”$ & METODO \\
\hline M1 & 32,3 & 29,8 & 36,47 & Ref/Tran u\&e \\
\hline M2 & 32,9 & 30,90 & 35,27 & Ref/Tran u\&e \\
\hline M3 & 30,5 & 25,28 & 17,41 & Ref/Tran u\&e \\
\hline M4 & 31,5 & 25,18 & 27,47 & Ref/Tran u\&e \\
\hline M5 & 33,9 & 24,10 & 22,39 & Ref/Tran u\&e \\
\hline M2 & 32,9 & 38,8 & 51,00 & Ref/Tran e Prec'r \\
\hline M2 & 32,9 & 27,3 & 39,00 & Ref/Tran e Fast \\
\hline \hline
\end{tabular}

En el software puede visualizarse el comportamiento tanto de la constante dieléctrica como del factor de perdidas. En la Figura 1-16 se observan los gráficos para la muestra 5, en todos los casos se observa que la constante dieléctrica no muestra una dependencia con la frecuencia mientras el factor de perdidas muestra claramente su relación inversa con la frecuencia.

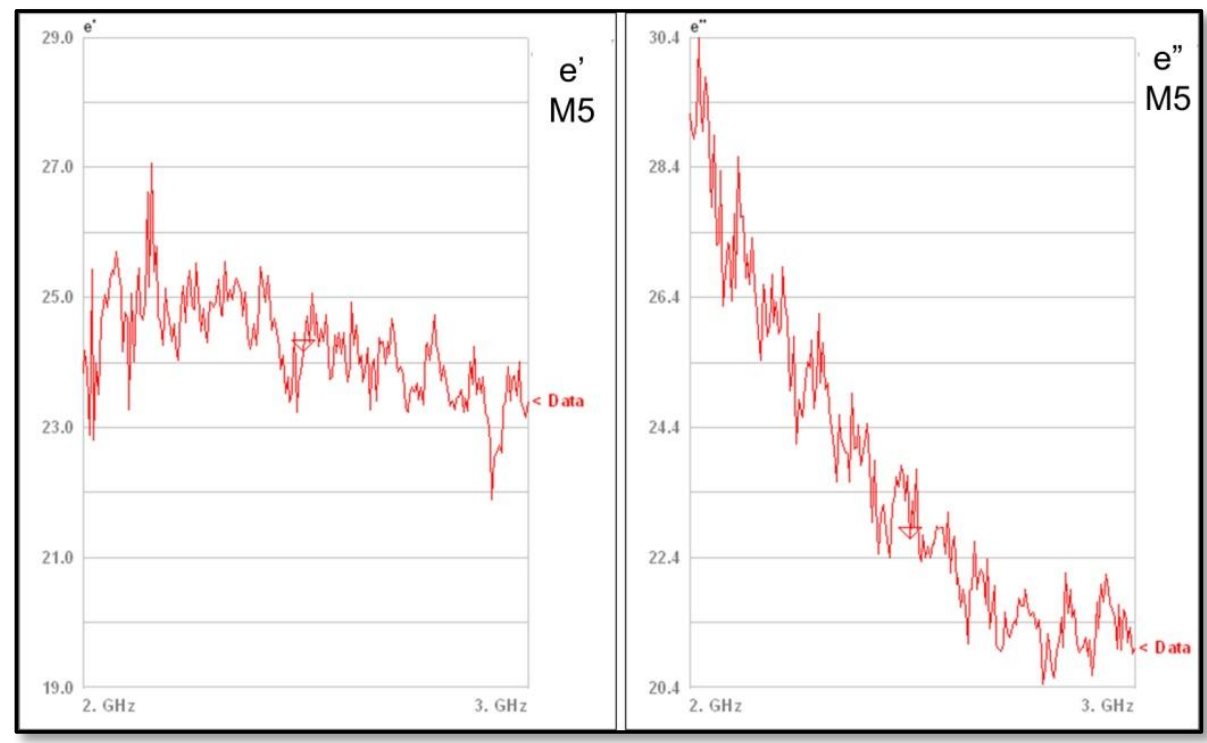

Figura 1-16. Permitividad vs. Frecuencia

Puesto que el programa supone una muestra homogénea lo que se obtiene como resultado es una permitividad efectiva de toda la muestra. Las muestras M1 y M2 son las que tienen menos grasa por eso sus propiedades son más altas que las de de las muestras M3, M4 y M5 que tienen partes claramente grasas. 
La variación presentada entre las propiedades de muestras similares es menor que la presente entre los diferentes modelos con la misma muestra. Se ha utilizado mayoritariamente el método de reflexión y transmisión de Nicolson-Ross con el objetivo de confirmar que las propiedades magnéticas sean efectivamente 1-j0.

Los resultados obtenidos son más cercanos a los encontrados en la literatura. La mediana de la contante dieléctrica es 25,28 con un índice de dispersión de 13\%, mientras la del factor de pérdidas es 27,47 con un índice de $27 \%$. De manera general el jamón curado tiene constante dieléctrica menor que los jamones de la literatura y superior a 25, mientras el factor de pérdidas es mayor que el de la literatura y habitualmente mayor a 20 .

La variación entre las muestras depende en gran medida de la parte del jamón de donde se tome la muestra, ya que en el exterior siempre está más seco que el interior. En consecuencia las medidas realizadas se utilizan solo para demostrar la superficialidad de un tratamiento con microondas en piezas de jamón curado.

\subsection{CONCLUSIÓN}

Se han conseguido los valores de las propiedades dieléctricas de la grasa tanto fresca como curada. Se observó que tanto la constante dieléctrica como el factor de perdidas disminuyen con el proceso de cura del jamón, ésta disminución se atribuye mayoritariamente al proceso de secado. La variación de las propiedades dieléctricas de la grasa no es significativa para el proceso de calentamiento, debido a que el grosor de la capa de grasa es mucho más pequeño que la profundidad de penetración de la misma, no afectando de esta manera el tratamiento con microondas.

En relación con el magro se obtuvo un valor orientativo para las propiedades dieléctricas del jamón curado. La constante dieléctrica disminuye notablemente con el proceso de cura, mientras el factor de perdidas aumenta. Dicho comportamiento se atribuye mayoritariamente al efecto de la absorción de sal durante el proceso de cura. Las altas perdidas obtenidas y el hecho que sean mayores en la superficie debido al proceso de absorción de sal garantizan el tratamiento superficial cuando se aplican microondas.

Es importante resaltar que los jamones son anisótropos (ya que su composición hace que la $\varepsilon$ sea diferente en las dirección de las fibras y su ortogonal) y no homogéneos, por lo que los valores obtenidos sirven como un valor orientativo más que como un valor exacto, y el planteamiento analítico del problema planteado es muy complejo. 


\section{CAPÍTULO 2. DESINSECTACIÓN DE JAMÓN CURADO}

\subsection{METODOLOGÍA}

En este capítulo se demuestra la viabilidad de erradicar los ácaros del jamón curado mediante la aplicación de microondas, estableciendo combinaciones de potenciatiempo que eliminen los ácaros sin afectar al jamón ni a sus propiedades organolépticas. Para esto es necesario disponer de trozos de jamón curado, ácaros, sensores de temperatura y un aplicador de microondas.

Los trozos de jamón curado proceden de la parte denominada maza, que ofrece normalmente un mayor grado de infiltración de grasa, es una zona muy apreciada sensorialmente y ofrece bastante rendimiento. Los ácaros (Tyroglyphus) se encuentran en jamones infestados naturalmente, luego son cultivados in vitro en el laboratorio y se mantienen en un cultivo de jamón con una temperatura de $27^{\circ} \mathrm{C}$ y una humedad relativa de $70 \%$.

Los sensores de temperatura son de fibra óptica. Estas sondas pueden introducirse dentro del horno y ser controladas desde el exterior del mismo. Adicionalmente, no constituyen un problema en relación con la fuga de microondas y no afectan de ninguna manera el proceso de calentamiento en el interior del horno.

El aplicador es un horno de instrumentación de microondas multimodo, con una potencia aproximada de $2,3 \mathrm{~kW}$, con un control de potencia regulable de forma analógica y un control digital de tiempo, ubicados ambos en la parte frontal del mismo. Cuenta con stirrer y plato giratorio para homogenizar el campo.

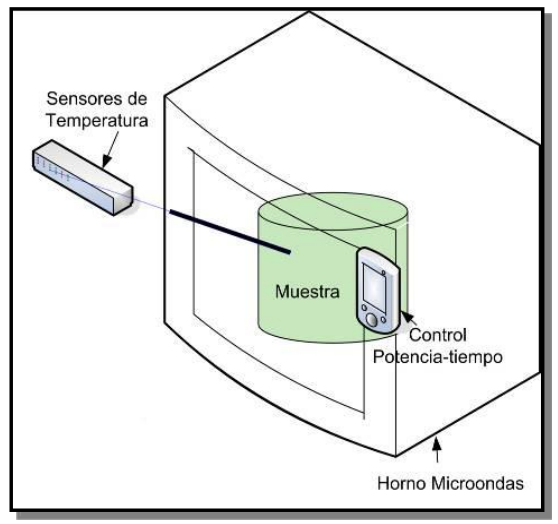

Figura 2-1. Configuración - experimentos en trozos de jamón 
La experimentación con trozos de jamón se divide en dos etapas: la primera de selección de tratamiento y la segunda de verificación del tratamiento. En ambas etapas se utiliza la configuración mostrada en la Figura 2-1.

La selección del tratamiento busca identificar aquellos tratamientos en los que la temperatura del jamón esté por debajo de $40^{\circ} \mathrm{C}$, nivel de temperatura admisible para conservar el jamón en buenas condiciones, y que a su vez permitan la eliminación de los ácaros. Las muestras consisten en un trozo de jamón curado con un peso aproximado de $1 \mathrm{~kg}$ y una placa petri con ácaros en su interior situada en la superficie. No se activa ni el plato giratorio ni el stirrer para contemplar el caso peor.

Como paso previo se trata de establecer la energía necesaria para alcanzar los $40^{\circ} \mathrm{C}$ en cualquier punto del jamón. Para esto se colocan las sondas de temperatura en tres puntos:

1. Superficie: la punta de la sonda es pegada con cinta adhesiva en la superficie del jamón.

2. Magro: se hace un agujero en la parte magra del jamón y se introduce la sonda.

3. Grasa: se ubica la sonda en el interior de una zona de abundante grasa.

A continuación se aplican 30 segundos de microondas a diferentes potencias, hasta encontrar la combinación que permite al jamón alcanzar los $40^{\circ} \mathrm{C}$, esta energía se establece como límite para los tratamientos de las muestras con ácaros en placas petri.

A partir de aquí se establece un conjunto de tratamientos (combinación potencia tiempo) respetando el límite anterior. Cada uno de estos tratamientos es aplicado en dos muestras mientras se controla la temperatura del jamón. Una vez terminado el experimento se llevan las placas petri al laboratorio, los ácaros son introducidos en un cultivo de jamón igual al de la población de control, son conservados en las mismas condiciones que ésta y observados durante 20 días para evaluar la efectividad del tratamiento. Por otro lado, los trozos de jamón son llevados al laboratorio para hacer una prueba de valoración sensorial, en la que se confirme que sus propiedades organolépticas siguen siendo las deseadas.

En la verificación del tratamiento se escogen los tratamientos efectivos de la etapa anterior para aplicarlos en trozos de jamón infestados naturalmente. En este caso se activará el stirrer para la aplicación de dichos tratamientos. Con el fin de obtener las muestras se escoge un jamón infestado de ácaros, luego se divide en seis partes (Figura 2-2), y finalmente la mitad de las muestras es tratada con los tratamientos escogidos y la otra mitad es utilizada como población de control. Después de 20 días se observan las diferencias entre las muestras tratadas y las no tratadas para evaluar la efectividad de los tratamientos en un entorno más real. 


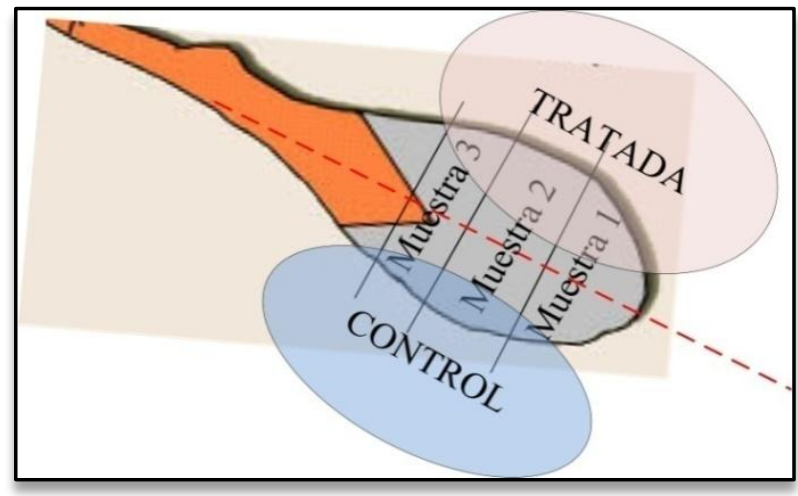

Figura 2-2.Muestras verificación del tratamiento

Por último se hacen pruebas preliminares en seis piezas enteras de jamón con el objetivo de dar un primer paso hacia una escala industrial. En este caso se debe cambiar el aplicador, debido al gran tamaño de las muestras. El objetivo es conseguir una orientación sobre el método más adecuado de aplicación. Sin embargo, el establecer procedimientos de aplicación exactos y el diseño del aplicador se deja para futuras investigaciones.

El aplicador utilizado fue diseñado para el tratamiento de tierras con una aplicación continua de microondas. El horno consta de una región activa en forma de túnel, una red de alimentación y filtros de microondas que permiten el paso del material pero evitan fugas hacia el usuario.

El material a tratar se mueve por la región activa (1,5 m de longitud) mediante una cinta transportadora (de un material transparente a las microondas), mientras es irradiado durante el tiempo de tratamiento. La red de alimentación está compuesta por cuatro magnetrones, que pueden ser activados independientemente y cada uno proporciona $1 \mathrm{~kW}$ de potencia. Los filtros están a la entrada y salida del túnel.

El tratamiento a aplicar es de $2 \mathrm{~kW}$ durante 30 o 60 segundos, de modo que sólo se activan los dos primeros magnetrones (Figura 2-3), que son los que cubren el espacio ocupado por la pieza de jamón y la cinta transportadora no se pone en funcionamiento. Adicionalmente, se cierra la entrada y salida del túnel con placas conductoras antes de empezar el tratamiento por lo que los filtros no son necesarios.

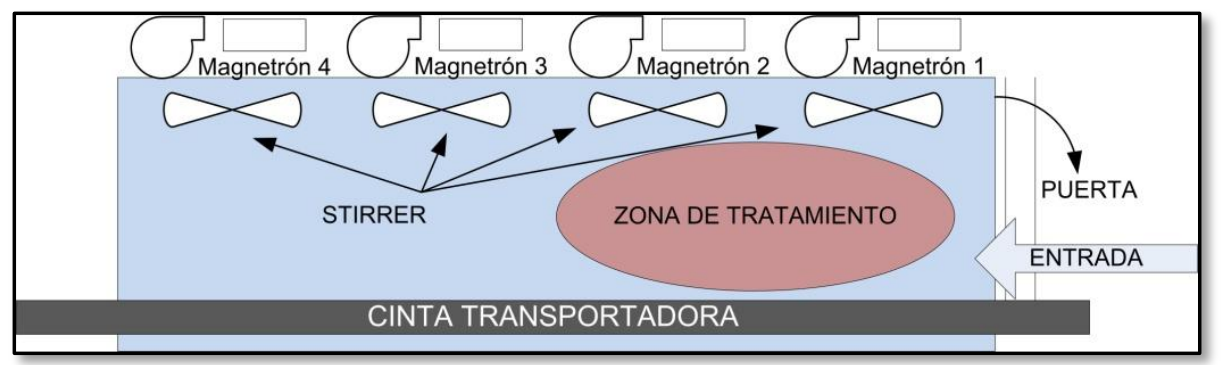

Figura 2-3. Aplicador para piezas enteras 


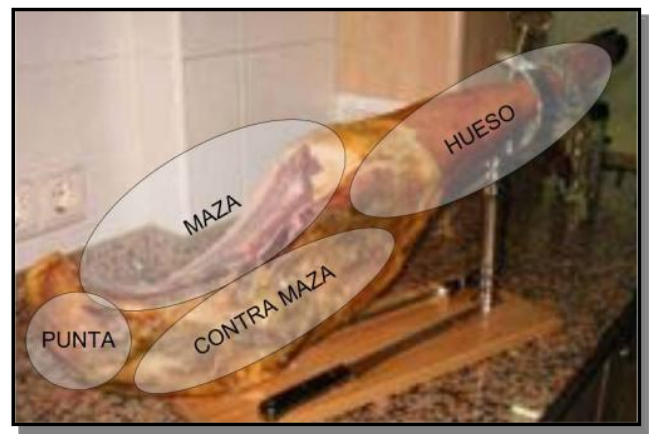

Figura 2-4. Partes de la pieza de jamón

El tratamiento de cada pieza de jamón se hace teniendo en cuenta la ubicación de la misma dentro del horno según sus partes (Figura 2-4), para esto se definen dos variables:

1. La orientación indica la disposición de la maza en el eje del hueso y tiene dos posibles valores:

- Arriba cuando la maza está orientada hacia el stirrer y

- abajo cuando la contra maza está orientada hacia el stirrer.

2. La dirección indica la forma en que se introduce el jamón en el horno independientemente de su orientación, son dos casos:

- A cuando la pieza se introduce en el horno con el hueso en dirección a la puerta, $\mathrm{y}$

- B cuando la pieza se introduce en el horno con la punta en dirección la puerta.

Adicionalmente, se colocan dos sondas de temperatura para medir la temperatura inicial de la pieza y la que alcanza después del tratamiento. La primera de estas sondas está colocada sobre la superficie del magro en la parte de la maza de la pieza y la segunda está en la contra maza, introducida en una zona de abundante grasa.

Una vez tratados, los jamones se identifican con códigos y se empaquetan en plásticos individuales para evitar el intercambio de ácaros entre las diferentes piezas. Luego son llevados a la planta de secado donde se conservan en un entorno adecuado y después de dos meses se observa el efecto del tratamiento. Como se ha mencionado anteriormente éstas son unas medidas preliminares y por eso no se han realizado con todo el rigor de una investigación orientada a escala industrial lo cual se deja para futuras investigaciones.

\subsection{SELECCIÓN DE TRATAMIENTO}

Se cuenta con dos trozos de jamón más o menos de forma cúbica con lados de 10 $\mathrm{cm}$, estas dos muestras pesan 1270,5 y 902,7 g cada una y se denominan M1 y M2 respectivamente. 
La sonda de temperatura correspondiente al magro se sitúa de manera diferente en cada muestra sobre una de las caras, estando introducida a una profundidad de 3,8 $\mathrm{cm}$ en la dirección Z (Figura 2-5). La ubicación de la sonda en la Muestra M1 es de $5 \mathrm{~cm}$ en la dirección X y $4 \mathrm{~cm}$ en la $\mathrm{Y}$, en el caso de la muestra M2 la sonda se encuentra a 1,5 y $3 \mathrm{~cm}$ en $\mathrm{X}$ y $\mathrm{Y}$ respectivamente.

Las muestras son pesadas antes y después del tratamiento, como resultado se tiene que el peso de las muestras no varía significativamente después de la aplicación de microondas, obteniendo variaciones de $0,1 \mathrm{~g}(<0,01 \%)$.

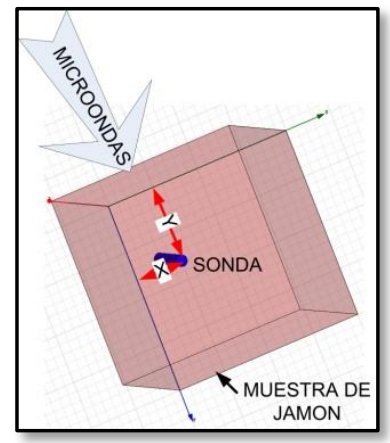

Figura 2-5. Ubicación de la sonda en el magro

En la Tabla 2-1 se muestran los resultados de temperatura para diferentes tratamientos. Como primer resultado se puede afirmar que el tratamiento efectivamente es superficial; ya que la temperatura en el interior del jamón no aumenta significativamente más de $1{ }^{\circ} \mathrm{C}$. Por su parte, la temperatura en la grasa aumenta entre 3 y $7{ }^{\circ} \mathrm{C}$ pero difícilmente superara los $25^{\circ} \mathrm{C}$, y la temperatura de la superficie aumenta significativamente por encima de los $10^{\circ} \mathrm{C}$ hasta los $18^{\circ} \mathrm{C}$.

Tabla 2-1. Temperatura en trozos de jamón

\begin{tabular}{||c|c|c|c|c|c||}
\hline \multicolumn{2}{|c|}{ Muestra } & M1 & M2 & M1 & M2 \\
\hline \multicolumn{2}{|c|}{ Potencia (\%) } & 5 & 10 & 20 & 15 \\
\hline \multicolumn{2}{|c|}{ Potencia Incidente (W) } & 120 & 230 & 420 & 320 \\
\hline \multicolumn{2}{|c|}{ Tiempo (s) } & 30 & 30 & 28 & 30 \\
\hline $\begin{array}{c}\text { Temperatura } \\
\left.\text { Superficie ( }{ }^{\circ} \mathbf{C}\right)\end{array}$ & Inicial & 16,45 & 16,78 & 19,6 & 20,87 \\
\cline { 2 - 6 } & Final & 26,92 & 26,46 & 41,46 & 31,12 \\
\hline $\begin{array}{c}\text { Temperatura } \\
\left.\text { Magro ( }{ }^{\circ} \mathbf{C}\right)\end{array}$ & Inicial & 10,21 & 12,97 & 12,76 & 17,83 \\
\cline { 2 - 6 } & Final & 10,25 & 13,16 & 13,9 & 19,2 \\
\hline \multirow{2}{*}{$\begin{array}{c}\text { Temperatura } \\
\text { Grasa }\left({ }^{\circ} \mathbf{C}\right)\end{array}$} & Inicial & 13,8 & 15,97 & 17,54 & 19,63 \\
\cline { 2 - 6 } & Final & 16,34 & 20,09 & 24,11 & 26,7 \\
\hline \hline
\end{tabular}


La energía aplicada para alcanzar $\operatorname{los} 41,46^{\circ} \mathrm{C}$ de la Tabla 2-1 es $11760 \mathrm{~J}$. A pesar que el calentamiento lo genera la potencia absorbida más que la potencia aplicada, y la potencia absorbida depende de las propiedades de cada jamón que varían con la temperatura, se utiliza ésta energía como límite para establecer los diferentes tratamientos a aplicar en muestras con ácaros en placas petri.

Tabla 2-2.Tratamientos y supervivencia.

\begin{tabular}{|c|c|c|c|c|c|c|c|c|c|c|}
\hline \multicolumn{4}{|c|}{ TRATAMIENTO } & \multicolumn{6}{|c|}{ TEMPERATURA $\left({ }^{\circ} \mathrm{C}\right)$} & \multirow{3}{*}{$\begin{array}{c}\text { MUSTRA Y } \\
\text { SUPER- } \\
\text { VIVENCIA }\end{array}$} \\
\hline \multirow[b]{2}{*}{ ID } & \multirow{2}{*}{$\begin{array}{c}\mathrm{t} \\
(\mathrm{s})\end{array}$} & \multicolumn{2}{|c|}{ Potencia } & \multicolumn{2}{|c|}{ Superficie } & \multicolumn{2}{|c|}{ Magro } & \multicolumn{2}{|c|}{ Grasa } & \\
\hline & & $\%$ & $\begin{array}{l}\mathrm{Pi} \\
\mathrm{W}\end{array}$ & $\mathrm{T}_{0}$ & $\mathrm{~T}_{\mathrm{f}}$ & $\mathrm{T}_{0}$ & $\mathrm{~T}_{\mathrm{f}}$ & $\mathrm{T}_{0}$ & $\mathrm{~T}_{\mathrm{f}}$ & \\
\hline 1 & 25 & 15 & 420 & 21,3 & 30,7 & 19,4 & 22,2 & 21,6 & 25,4 & M2 - 0 \\
\hline 2 & 25 & 15 & 420 & 20,7 & 38,7 & 16,5 & 16,7 & 22,0 & 27,9 & M1 - 0 \\
\hline 3 & 28 & 15 & 420 & 23,1 & 34,9 & 21,4 & 23,4 & 24,0 & 25,6 & M2 - 0,05 \\
\hline 4 & 28 & 15 & 420 & 26,0 & 36,9 & 23,0 & 24,7 & 24,0 & 26,5 & M2 - 0,05 \\
\hline 5 & 44 & 10 & 220 & 21,4 & 45,0 & 17,6 & 19,0 & 22,6 & 26,8 & M1 - 0,125 \\
\hline 6 & 46 & 10 & 220 & 28,0 & 41,7 & 24,0 & 26,6 & 26,0 & 27,3 & M2 - 0 \\
\hline 7 & 15 & 40 & 600 & 23,5 & 40,5 & 20,0 & 19,9 & 24,9 & 30,3 & M1 - 0,05 \\
\hline 8 & 15 & 40 & 600 & 29,9 & 38,4 & 25,6 & 26,8 & 26,5 & 28,5 & M2 - 0,05 \\
\hline 9 & 15 & 45 & 640 & 25,0 & 44,6 & 20,4 & 20,7 & 26,4 & 30,4 & M1 - 0,1 \\
\hline 10 & 15 & 45 & 640 & 28,5 & 37,7 & 25,6 & 26,8 & 27,2 & 28,8 & M2 - 0,1 \\
\hline 11 & 15 & 50 & 700 & 26,0 & 47,8 & 21,6 & 21,6 & 27,0 & 31,0 & M1 - 0,225 \\
\hline 12 & 15 & 50 & 680 & 31,3 & 45,1 & 26,9 & 28,5 & 27,7 & 29,0 & M2 - 0,45 \\
\hline 13 & 40 & 8 & 160 & 27,8 & 41,6 & 22,4 & 22,5 & 28,0 & 31,7 & M1 - 0 \\
\hline 14 & 40 & 8 & 160 & 29,9 & 37,1 & 27,8 & 29,1 & 27,8 & 28,8 & M2 - 0 \\
\hline 15 & 60 & 4 & 100 & 26,8 & 37,5 & 22,9 & 23,1 & 27,5 & 30,3 & M1 - 0 \\
\hline 16 & 90 & 2 & 50 & 30,4 & 34,9 & 27,8 & 28,9 & 28,1 & 28,9 & M2 - 0 \\
\hline 17 & 180 & 2 & 50 & 24,8 & 41,0 & 23,1 & 23,2 & 28,0 & 32,4 & M1 - 0,033 \\
\hline 18 & 120 & 5 & 120 & 30,2 & 42,7 & 27,7 & 30,3 & 28,1 & 32,8 & M2 - 0 \\
\hline
\end{tabular}

En la Tabla 2-2 se enumeran los 18 tratamientos aplicados, al igual que la temperatura en los tres puntos descritos y la efectividad del tratamiento, que está representada por la supervivencia de los ácaros, que a su vez está dada por:

$$
\text { Supervivencia }=\frac{\text { Acaros Vivos }}{\text { Acaros } \text { Tratados }}
$$


Con el objeto de comprobar el efecto de una exposición prolongada, el límite de energía aplicada propuesto se supera en el tratamiento número 18 , ya que en experimentos anteriores con arroz [22] se observó que los insectos pequeños buscan las zonas de menor campo eléctrico para refugiarse. En éste experimento se observa que los ácaros se mueven rápidamente y se refugian mayoritariamente en los extremos de la placa.

Los ácaros de la población de control no sufren ninguna baja después del tiempo de observación, mientras que la efectividad de los tratamientos es muy buena cuando los ácaros sufren exposiciones largas (superiores a 40 segundos) a baja potencia (inferior a $220 \mathrm{~W}$ ). La única excepción encontrada es el tratamiento número No. 17, no se aplican tratamientos de larga exposición a potencias altas ya que se superaría rápidamente el límite de temperatura en la superficie del jamón, fijado en $40^{\circ} \mathrm{C}$.

Por otro lado, se puede afirmar que la efectividad de los tratamientos no está relacionada con la energía aplicada o con la temperatura alcanzada en la superficie; ya que para la misma energía obtenida con diferentes combinaciones potenciatiempo se consiguen diferentes resultados, al igual que para temperaturas similares en la superficie (datos en la Tabla 2-2).

Los tratamientos a verificar en la siguiente fase son de larga duración a baja potencia:

1. Tratamiento número 6: $220 \mathrm{~W}$ durante 46 segundos.

2. Tratamiento número 18: $120 \mathrm{~W}$ durante 120 segundos.

3. Tratamiento extra: con el fin de comprobar la regla, 50W durante 240 segundos.

\subsection{VERFICACIÓN DEL TRATAMIENTO}

Se tratan tres muestras con los tratamientos anteriormente mencionados. Estas muestras son tomadas de un jamón infestado de ácaros según la Figura 2-2. Las muestras M3, M4 y M5 tienen un peso de 1086,6; 618 y 1475,1 g respectivamente.

Para la población de control se toman también tres muestras del mismo jamón y correspondientes al mismo corte de las muestras tratadas, denominándose C3, C4 y C5 respectivamente.

Tabla 2-3. Verificación de tratamientos

\begin{tabular}{||c|c|c|c|c|c||}
\hline \multirow{2}{*}{} & \multicolumn{2}{|c|}{ TRATAMIENTO } & \multicolumn{2}{|c|}{$\begin{array}{c}\text { TEMPRATURA } \\
\left({ }^{\circ} \text { C) }\right.\end{array}$} & \multirow{2}{*}{ SUPERVIVENCIA } \\
\cline { 2 - 5 } & $\mathrm{P}_{\mathrm{i}}(\mathrm{W})$ & $\mathrm{t}(\mathrm{s})$ & Inicial & Final & \\
\hline M3 & 220 & 46 & 19 & 29 & 0 \\
\hline M4 & 120 & 120 & 20 & 31 & 0 \\
\hline M5 & 50 & 240 & 19 & 31 & 0 \\
\hline
\end{tabular}


En la Tabla 2-3 se muestran los tratamientos aplicados y los resultados obtenidos en cuanto a temperatura y supervivencia. Se observa que la temperatura alcanzada en la superficie del jamón no es superior a $\operatorname{los} 31^{\circ} \mathrm{C}$, demostrando la seguridad del tratamiento con microondas en los tratamientos aplicados.

Los ácaros de los trozos tratados como los del control se observan después 20 días, los tratamientos de las muestras M3 y M4 se encuentran efectivos y están validados por el hecho que los ácaros de los controles C3 y C4 están en perfectas condiciones.

Por otro lado, la totalidad de los ácaros del control C5 murieron y aunque tardaron más en morir que los ácaros de la muestra M5, la erradicación de los ácaros se atribuye al mal estado del jamón en éste corte (tan cercano al hueso) más que al efecto de las microondas sobre los ácaros.

\subsubsection{Valoración sensorial}

El tratamiento aplicado para la realización de estas pruebas es de $50 \mathrm{~W}$ durante 240 segundos, ya que éste caso es el que degradaría en mayor medida el jamón. Las muestras utilizadas son la M6 y M7 con pesos de 448,4 y 512 g respectivamente.

En la Tabla 2-4 se observa la temperatura alcanzada en la superficie y el interior del jamón. Las temperaturas alcanzadas son superiores a las alcanzadas en los tratamientos verificados en la Tabla 2-3 y muy cercanas al límite propuesto para esta investigación, siendo por lo tanto los resultados de ésta valoración válidos para los tratamientos mencionados anteriormente.

Tabla 2-4. Temperatura de jamones en valoración sensorial.

\begin{tabular}{|l|c|c|c||}
\hline \hline & $\begin{array}{c}\text { Inicial } \\
\left({ }^{\mathbf{}} \mathbf{C}\right)\end{array}$ & $\begin{array}{c}\text { Final superficie } \\
\left({ }^{\mathbf{}} \mathbf{C}\right)\end{array}$ & $\begin{array}{c}\text { Final Interna } \\
\left({ }^{\mathbf{}} \mathbf{C}\right)\end{array}$ \\
\hline M6 & 12 & 44 & 22 \\
\hline M7 & 14 & 38 & 23 \\
\hline
\end{tabular}

La valoración consistió en dar a probar a diferentes sujetos lonchas de jamón surtidas de las muestras tratadas M6 y M7, y de jamones que no han sido tratados con microondas. El resultado fue que los sujetos no sienten diferencias significativas entre los jamones tratados y los no tratados. Las características valoradas fueron: sabor, olor, textura, aspecto y color. Este estudio fue llevado a cabo por el Departamento de Tecnología de Alimentos de la Universidad Politécnica de Valencia.

\subsection{TRATAMIENTO DE PIEZAS ENTERAS}

Se cuenta con seis piezas de aproximadamente $10 \mathrm{~kg}$ cada una. Cada pieza es identificada con un código de tres caracteres alfanuméricos en una etiqueta.

Debido al gran tamaño de las muestras a tratar y a que el calentamiento por microondas depende del volumen, los tratamientos validados anteriormente no se utilizan. Las muestras son tratadas con $2 \mathrm{~kW}$ de potencia durante el tiempo 
especificado en la Tabla 2-5, donde también se indica la temperatura alcanzada en la superficie y la grasa.

En cuanto a temperatura, el jamón está bajo los niveles seguros, ya que con el tratamiento de 30 segundos no se superan los $25{ }^{\circ} \mathrm{C}$ y con el de 60 segundos los $35^{\circ} \mathrm{C}$. De manera general, la temperatura alcanzada en la superficie del jamón es mayor que la alcanzada en la grasa, siendo de esta manera el tratamiento de $2 \mathrm{~kW}$ durante 30 o 60 segundos seguro para piezas de jamón enteras.

Tabla 2-5. Tratamiento piezas enteras

\begin{tabular}{|c|c|c|c|c|c|c|c|c|}
\hline \multicolumn{2}{|c|}{ MUESTRA } & \multicolumn{2}{|c|}{ POSICIÓN } & TRATAMIENTO & \multicolumn{4}{|c|}{ TEMPERATURA $\left({ }^{\circ} \mathrm{C}\right)$} \\
\hline \multirow{2}{*}{$\frac{\tilde{D}}{0}$} & \multirow{2}{*}{$\begin{array}{l}\stackrel{\widetilde{N}}{\stackrel{N}{2}} \\
\stackrel{\theta}{\theta}\end{array}$} & \multirow{2}{*}{ 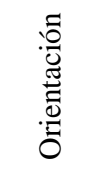 } & \multirow{2}{*}{ 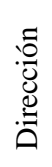 } & \multirow{2}{*}{$2 \mathrm{~kW}$ - Tiempo (s) } & \multicolumn{2}{|c|}{ Superficie } & \multicolumn{2}{|c|}{ Grasa } \\
\hline & & & & & Inicial & Final & Inicial & Final \\
\hline 1 & $47 d$ & Arriba & B & 60 & 17,9 & 33,0 & 16,1 & 19,7 \\
\hline 2 & $31 d$ & Abajo & B & 60 & 18,1 & 26,2 & 16,27 & 22,2 \\
\hline 3 & $48 \mathrm{~d}$ & Arriba & A & 30 & 18,2 & 21,9 & 16,0 & 18,9 \\
\hline 4 & $12 \mathrm{~d}$ & Arriba & A & 30 & 16,3 & 18,5 & 15,8 & 17,4 \\
\hline 5 & $82 d$ & Arriba & A & 30 & 17,9 & 24,0 & 15,9 & 18,2 \\
\hline 6 & $78 \mathrm{~d}$ & Abajo & A & 30 & 17,8 & 19,8 & 16,0 & 17,3 \\
\hline 7 & $48 \mathrm{~d}$ & Abajo & A & 30 & 18,9 & 20,7 & 18,9 & 22,9 \\
\hline 8 & $12 \mathrm{~d}$ & Abajo & A & 30 & 18,9 & 20,0 & 17,3 & 19,3 \\
\hline
\end{tabular}

En relación con el método de aplicación, se aconseja la orientación hacia arriba, en la que la temperatura alcanzada en la superficie es mayor. Con esta orientación también se evita el contacto directo de los ácaros con las paredes del aplicador. En cuanto a la dirección, no es posible sacar una conclusión definitiva, ya que no se cuenta con duraciones iguales para los dos tipos de dirección.

Adicionalmente, tanto la orientación como la dirección dependen en gran medida del diseño del aplicador y su distribución de campo. Un aplicador de microondas debe ser diseñado como un equipo a medida. El problema al que nos enfrentamos es que el horno disponible está diseñado para tratar tierras sobre la cinta transportadora, no para tratar objetos de forma arbitraria. Por lo tanto, no se puede asegurar la homogeneidad del campo en la zona de tratamiento del jamón. 
Después de 70 días se observan los jamones tratados, cuyos resultados se muestran en la Tabla 2-6. Las muestras que fueron tratadas dos veces presentan más ácaros que las demás, lo cual puede deberse a varios factores (por ejemplo la gran velocidad a la que se mueven los ácaros les permite buscar las zonas donde el campo es menor con lo que pueden haberse refugiado dentro de la parte no utilizada del aplicador o sus paredes). Adicionalmente, estas piezas quedaron en contacto con las demás mientras se enfriaban para el segundo tratamiento y durante este tiempo pudieron adquirir más ácaros.

Tabla 2-6. Observación Ácaros

\begin{tabular}{||c|c||}
\hline ID pieza & Observación 70 días \\
\hline $47 \mathrm{~d}$ & Presencia de ácaros dispersos \\
\hline $31 \mathrm{~d}$ & Se ha podrido \\
\hline $48 \mathrm{~d}$ & Presencia de gran cantidad de ácaros \\
\hline $12 \mathrm{~d}$ & Presencia de gran cantidad de ácaros \\
\hline $82 \mathrm{~d}$ & Se ha podrido \\
\hline $78 \mathrm{~d}$ & Presencia de ácaros dispersos \\
\hline
\end{tabular}

Con respecto a la orientación y la dirección no se puede sacar una conclusión debido a que dos piezas se dañaron. Lo que sí se puede afirmar es que las piezas que fueron tratadas sólo una vez presentan menor cantidad de ácaros después del tratamiento, ya que fueron aisladas de manera inmediata tras el tratamiento.

Finalmente, después de estos 70 días se realiza un segundo tratamiento de las piezas, esta vez para observar la respuesta inmediata, ya que la respuesta a largo plazo es difícilmente estimable debido al poco control que se tiene de las piezas una vez se han tratado. Esta segunda aplicación tiene una orientación hacia arriba y una dirección del tipo A. En la Tabla 2-7 se muestran el tiempo y la temperatura de cada muestra.

Las temperaturas alcanzadas cerca al hueso y en la superficie de la zona maza son muy cercanas, con lo que se demuestra una vez más que el tratamiento es superficial.

De manera inmediata se observa que la temperatura casi no varía en la zona de contra maza, que la grasa que cubre el jamón está bastante caliente al tacto y que cambia su textura, y los ácaros se ven afectados por el tratamiento ya que se encuentran agrupados y muestran un movimiento rápido y errático. 
Tabla 2-7. Segundo tratamiento piezas enteras

\begin{tabular}{|c|c|c|c|c|c|c|c|c|}
\hline \multicolumn{2}{|c|}{ MUESTRA } & \multirow{3}{*}{$\begin{array}{l}\text { TRATAMIENTO } \\
2 \mathrm{~kW}-\text { Tiempo (s) }\end{array}$} & \multicolumn{6}{|c|}{ TEMPERATURA } \\
\hline \multirow{2}{*}{ 胥 } & \multirow{2}{*}{ 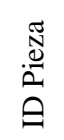 } & & \multicolumn{2}{|c|}{ Grasa } & \multicolumn{2}{|c|}{ Superficie } & \multicolumn{2}{|c|}{ Cerca al hueso } \\
\hline & & & Inicial & Final & Inicial & Final & Inicial & Final \\
\hline 1 & $31 d$ & 120 & 18,7 & & 19,5 & 52,0 & 18,9 & 37,0 \\
\hline 2 & $82 \mathrm{~d}$ & 90 & 17,9 & 26,3 & 19,2 & 48,0 & 18,8 & 52 \\
\hline 3 & $48 \mathrm{~d}$ & 120 & 19,45 & 30,4 & 20,2 & 34 & 20,1 & 33,0 \\
\hline 4 & $47 d$ & 90 & 19,5 & 24,7 & 20,3 & 35,1 & 20,1 & 47,2 \\
\hline 5 & $78 \mathrm{~d}$ & 60 & 19,8 & 25,8 & 20,5 & 27,2 & 20,1 & 27,3 \\
\hline 6 & $12 \mathrm{~d}$ & 60 & 19,6 & 29 & 20,6 & 27,4 & 20,7 & 29,4 \\
\hline
\end{tabular}

\subsection{CONCLUSIÓN}

A nivel de laboratorio se ha demostrado la viabilidad de la desacarización de jamón curado mediante microondas en trozos infestados tanto natural como artificialmente. Se encontró que con exposiciones prolongadas a bajas potencias de microondas los ácaros no sobreviven al tratamiento y que la temperatura alcanzada en la superficie del jamón no tiene relación con la supervivencia. En resumen, a exposiciones prolongadas con baja potencia se salvaguarda el jamón mientras se erradican los ácaros.

En relación con la experimentación a escala industrial se puede afirmar que es necesario el diseño de un aplicador con un campo homogéneo para la forma de los jamones y su tamaño. También es muy importante tener en cuenta sistemas de movimiento y aislamiento para evitar la fuga de los ácaros al tratamiento o que se refugien en zonas de campo eléctrico nulo. A esta escala es muy importante el diseño de experimentos y la forma de controlar los ácaros debido a su reducido tamaño y facilidad de movimiento. 


\section{SEGUNDA PARTE: Tecnología de microondas en el sector de restauración de bienes culturales}

Carcoma es el nombre común de diversas especies de escarabajos (Anobium punctatum, Xestobium rufovillosum, Lyctus brunneus,...) que producen daños severos en edificios, mobiliario y obras de arte. La última estimación conocida en Estados Unidos del coste anual del control y restauración de los daños producidos por insectos xilófagos es de 1996, y ascendía a más de mil millones de dólares USA. En Europa, aunque no se conocen cifras globales, existe una preocupación palpable por este problema, incrementada por el impacto potencial en el inestimable patrimonio artístico e histórico del continente.

Aunque la vida de los insectos xilófagos es realmente breve (1-2 semanas), los insectos adultos depositan sus huevos en grietas y oquedades de la madera, donde eclosionan al cabo de una o dos semanas. Las larvas de estos insectos viven entre 5 y 7 años en el interior de la madera hasta alcanzar el estado de pupa, tras el cual se metamorfosean en adultos y abandonan la madera a través de galerías excavadas en la madera hacia la superficie de ésta. Adicionalmente, durante todo el proceso de crecimiento, las larvas se alimentan de la madera, formando galerías que pueden producir graves daños estructurales, llegando a destruir elementos de madera como vigas, techos, retablos, tablas o marcos.

Los métodos de desinsectación de madera se pueden agrupar en dos categorías principales: químicos y físicos. En la literatura especializada [23] se pueden encontrar comparaciones detalladas de los métodos comúnmente utilizados.

Los métodos químicos de desinsectación se basan en el uso de substancias venenosas que pueden ser fumigadas o aplicadas directamente en la madera a través de agujeros taladrados en ésta. Entre las sustancias fumigadas, las más populares han sido, tradicionalmente, el bromuro de metilo y el fluoruro de azufre. Sin embargo, hoy en día existe una preocupación cada vez mayor respecto a este tipo de substancias y algunas de ellas han sido incluso prohibidas en muchos países (en particular, el bromuro de metilo, debido a su efecto sobre la capa de ozono). Entre los productos químicos que deben ser aplicados directamente sobre la madera cabe destacar el pyrethrum en aerosol y los pyrethroid líquidos y en aerosol (cyfluthrin, permethrin, bifenthrin), imidacloprid líquido, nitrógeno líquido y formulaciones líquidas y en polvo de perborato de sodio. Todas estas substancias son altamente tóxicas y deben ser cuidadosamente manipuladas por personal debidamente cualificado y pueden, además, dejar restos tras el tratamiento.

Un procedimiento general para la aplicación de insecticidas es inyectarlos a la máxima profundidad por los orificios de salida mediante una jeringuilla. Luego se enmasilla la obra lo cual intensifica la eficacia del insecticida, impide una 
volatilización rápida, dificulta el desove de los insectos dañinos y controla los efectos. En cuanto a la fumigación con biocidas gaseiformes o con gases inertes se logra una eficacia inmediata y radical, pero no sirve como método preventivo ya que el cuadro puede sufrir nuevas invasiones al poco tiempo [24].

Por todo lo anteriormente expuesto, el público en general viene demostrando en los últimos tiempos un interés cada vez mayor en los métodos de desinfección no químicos. Entre los métodos de desinfección no químicos hay que destacar el calor extremo, el frío extremo, la electrocución, los rayos X y gamma, y las microondas.

Los métodos de calor extremo se basan en elevar la temperatura de la madera por encima del límite vital de los insectos; estos métodos son efectivos si la temperatura de la madera se mantiene por encima de $\operatorname{los} 55^{\circ} \mathrm{C}$ al menos durante 33 minutos. Sin embargo, los métodos basados en calor pueden producir daños en la madera y no son en absoluto válidos para determinados elementos, como puedan ser policromías o tablas pintadas.

Específicamente en el método del aire caliente (permitido desde 1951 y que alcanzó su mayor importancia a mediados de los 50's) se introducía aire a 100-120 ${ }^{\circ} \mathrm{C}$ en una habitación donde se encontraba la madera afectada, que se mantenía caliente durante un mínimo de 60 minutos a una temperatura no inferior a $55^{\circ} \mathrm{C}$ con lo que morían los insectos. Adicionalmente, la humedad del aire se regulaba con el fin de mantener la humedad de la madera, de éste modo no se produce intercambio de humedad entre la madera y su entorno, y por lo tanto no se presenta en la madera una reacción que podría dar lugar a un levantamiento de capas.

Los métodos basados en frío extremo tienen un principio de funcionamiento análogo: se basan en reducir la temperatura del objeto que se quiere desinsectar por debajo de los valores en los cuales la vida del insecto es posible. En la actualidad hay métodos basados en frío que han demostrado ser eficaces en el tratamiento de piezas pequeñas, pero no son viables para tratar in situ elementos grandes (vigas, artesonados, retablos) ya que hay que introducirlos en recintos en cuyo interior se pueda mantener una temperatura suficientemente baja.

Por lo que respecta a la electrocución, ésta se basa en la inyección de corrientes de alto voltaje y baja intensidad de corriente, pero su eficacia es muy reducida (del orden de un $44 \%$ ).

La carcoma es muy resistente a los efectos de las radiaciones con rayos $\mathrm{X}$ y gamma, para matarla se requiere una dosis mucho más alta que para los mamíferos. Además los pigmentos de plomo cambian visiblemente con una dosis de sólo 100000 roentgen por lo que se considera un método no adecuado para la desinsectación de obras de arte [24].

Por su parte, la energía de microondas ha sido propuesta como una alternativa a los pesticidas químicos en productos agrícolas desde hace ya tiempo [25-28]. La efectividad de las microondas contra los insectos parásitos se debe a la diferencia extrema en el contenido de agua del grano ( 10\%) y los insectos $(>80 \%)$, lo que produce que la temperatura del insecto se eleve a niveles letales. 
En los noventa, Andreuccetti et al. comprobaron la efectividad de las microondas para desinsectar madera atacada por carcoma [29-31], después su trabajo fue exitosamente aplicado en desinsectación de tableros artísticos pintados [32, 33].

La viabilidad de desinsectar elementos constructivos mediante microondas ha sido explorada experimentalmente por Lewis y Haverty [23], en este trabajo se usaron tableros infestados tanto natural como artificialmente y con el fin de simular las condiciones de campo se construyó un edificio específicamente para este experimento. Se usaron varios tamaños de tablero; se aplicaron microondas a tableros de 10,2x30,5 cm usando un generador de potencia de $700 \mathrm{~W}$. Lewis y Haverty observaron una variabilidad considerable en la mortalidad, los valores ocasionalmente excedían el $90 \%$ en las pruebas sobre tableros infestados artificialmente, mientras en tableros infestados naturalmente la mortalidad media alcanzo valores sobre $98 \%$. Finalmente, concluyeron que las microondas parecen ser un método prometedor de desinsectación pero también resaltaron la necesidad de un estudio detallado sobre la penetración de energía electromagnética dentro de la madera y la determinación de la dosis letal exacta.

En un trabajo anteriormente realizado por el Grupo de Electromagnetismo Aplicado (GEA) de la Universidad Politécnica de Valencia (UPV) se diseñó un prototipo de sistema para desinsectación por microondas cuya polarización es lineal. El cual se probó en el laboratorio para verificar que a temperatura ambiente el patrón fuera tan uniforme como el de las simulaciones. Finalmente, se uso el prototipo y se verificó que cumpliera las recomendaciones internacionales [34], europeas [35] y españolas [36], limitando la exposición de las personas a campos electromagnéticos.

El problema a tratar en ésta parte de la tesis está compuesto tanto por la carcoma como por la madera. Hasta ahora se ha hablado ampliamente de la carcoma, pero es fundamental tener en cuenta la composición de la madera para que el tratamiento con microondas no la afecte. El objetivo es buscar un tratamiento y método de aplicación que permita erradicar la carcoma de obras de arte mientras éstas permanecen en su estado normal, conservando así el patrimonio artístico.

La madera es un material anisótropo cuyas propiedades dieléctricas han sido ampliamente estudiadas, ésta anisotropía se debe a la combinación desde la estructura molecular de celulosa hasta la estructura de las fibras dentro de la madera [37].

En relación con las propiedades dieléctricas de la madera, el tensor dieléctrico tiene sólo valores en las tres principales direcciones a lo largo de la diagonal de la matriz (i), donde $\varepsilon_{\mathrm{L}}$ es a lo largo de la dirección longitudinal (paralelo a las fibras), $\varepsilon_{\mathrm{R}}$ en la dirección radial y $\varepsilon_{\mathrm{T}}$ en la dirección tangencial, dando estas dos direcciones en relación con los anillos anulares de la madera y la longitudinal a lo largo de las fibras [38].

$$
\varepsilon^{\prime(\omega)}=\left(\begin{array}{ccc}
\varepsilon_{T}(\omega) & 0 & 0 \\
0 & \varepsilon_{L}(\omega) & 0 \\
0 & 0 & \varepsilon_{R}(\omega)
\end{array}\right)
$$


La relación de estos elementos está dada por $\varepsilon_{T} \leq \varepsilon_{R}<\varepsilon_{L}$ y debido a que $\varepsilon_{T} \approx \varepsilon_{R}$ se aproxima a $\varepsilon_{R T}$. En relación con la permeabilidad, la madera es un material no magnético.

Esta parte de la tesis se aborda desde la primicia que las pérdidas dieléctricas de la madera son menores que las de la carcoma, debido en gran parte a su contenido de agua, por lo que con una aplicación simultánea de microondas a ambos materiales se alcanzará primero mayores temperaturas sobre la carcoma que sobre la madera, permitiendo de esta manera eliminar la carcoma mientras se mantiene a salvo la madera.

En cuanto al método de aplicación es importante tener en cuenta la estructura de la madera y su anisotropía, para optimizar el uso de la energía de microondas y la potencia requerida. 


\section{CAPÍTULO 3. DESINSECTACIÓN DE RETABLOS}

\subsection{METODOLOGÍA}

En este capítulo se consigue un procedimiento (combinación potencia-tiempo y modo de aplicación) que permite la desinsectación de madera y obras de arte, empleando los materiales enumerados en la Tabla 3-1 y teniendo en cuenta los siguientes criterios:

1. Los valores de temperatura que permiten salvaguardar las características de la madera y la cara pintada. Estos límites se fijan en $55^{\circ} \mathrm{C}$ para la cara pintada $[24,32]$ y $60{ }^{\circ} \mathrm{C}$ en el interior de la madera según temperaturas alcanzadas en algunos tratamientos comerciales [24].

2. Los datos de mortalidad con diferentes tratamientos. Se define tratamiento como la exposición de una tabla con carcoma a una radiación de microondas de $900 \mathrm{~W}$ durante un período de tiempo determinado.

3. El efecto de las microondas sobre la madera y la imprimación. La imprimación es la capa sobre la cual se realiza la pintura, formada por una mezcla de cola orgánica con sulfato de calcio o carbonato, que ha sido previamente aplicada y secada.

Tabla 3-1. Materiales empleados y sus características

\begin{tabular}{||l|l||}
\hline \hline $\begin{array}{l}\text { Prototipo de un sistema de } \\
\text { desinsectación portátil [39] }\end{array}$ & $\begin{array}{l}\text { Funciona a 2,45 GHz. Cuenta con dos } \\
\text { magnetrones de 900 W enfriados con aire que } \\
\text { pueden activarse independientemente, } \\
\text { permitiendo seleccionar entre 900 y 1800 W de } \\
\text { potencia. } \\
\text { Los magnetrones se alimentan con una fuente } \\
\text { lineal de potencia, que suministra 4,000 V de y } \\
\text { se conecta a la red de 50 Hz. El switch de } \\
\text { encendido tiene una extensión de 5 m. } \\
\text { Los magnetrones y el aplicador están conectados } \\
\text { mediante cables coaxiales de alta potencia, que } \\
\text { pueden soportar 1,5 kW (a 2,45 GHz con una } \\
\text { operación CW). } \\
\text { Cuenta con una trampa de radio frecuencia para } \\
\text { mantener los niveles de radiación por debajo de } \\
\text { los límites permitidos [36]. }\end{array}$ \\
\hline
\end{tabular}

Tecnología de microondas en el sector de restauración de bienes culturales 


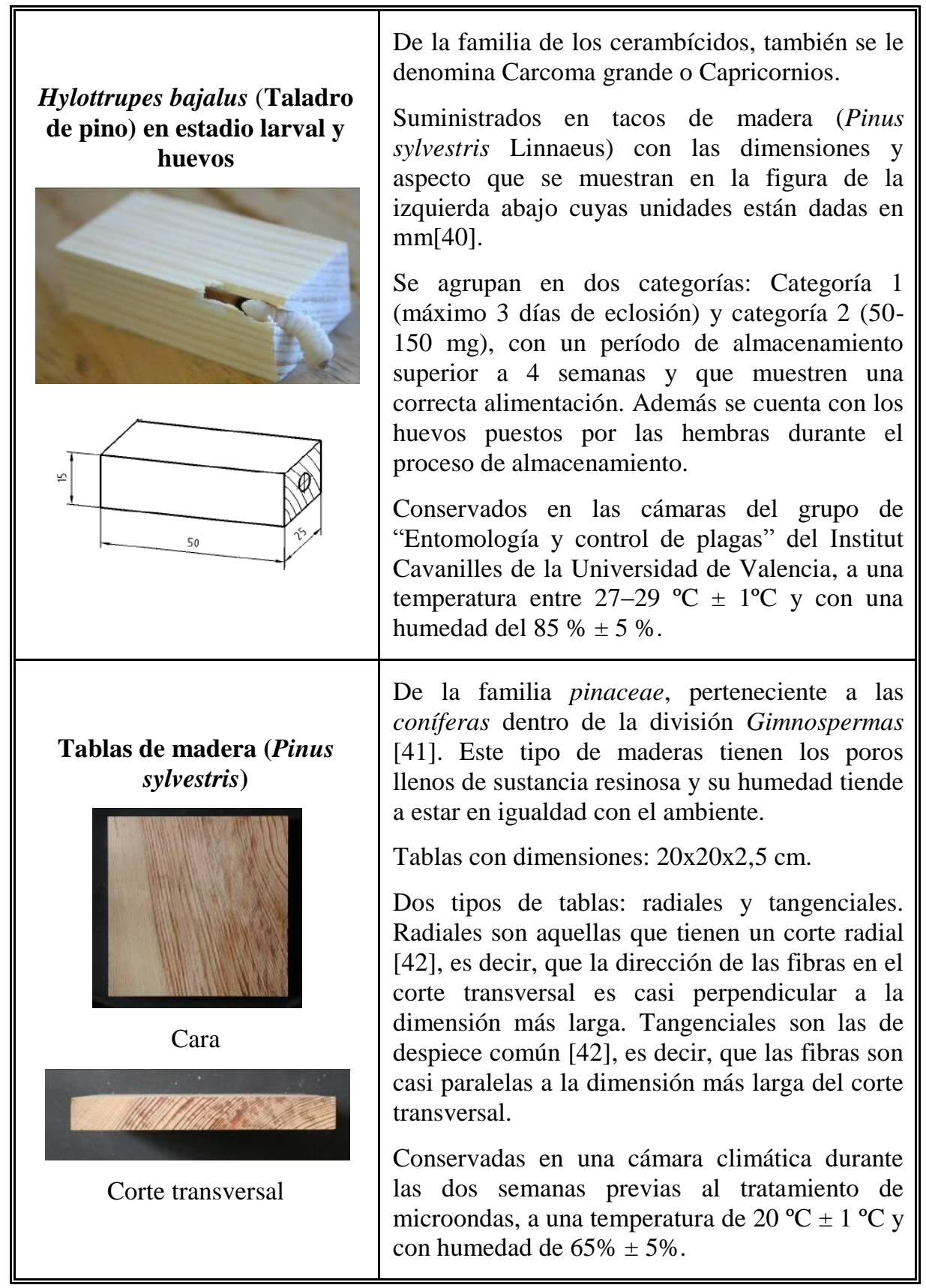

La Figura 3-1 muestra la configuración de la experimentación. Los experimentos se realizan dentro de una cámara apantallada como medio extra de protección contra la radiación. Esta área de ensayos se encuentra a una temperatura de $21-23{ }^{\circ} \mathrm{C}$ y con una humedad del 70-75\%.

42 Tecnología de microondas en el sector de restauración de bienes culturales 


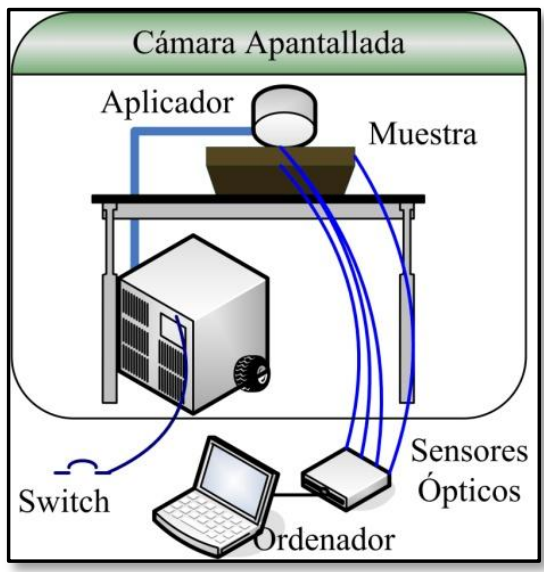

Figura 3-1. Configuración para la experimentación

El sistema de desinsectación se pone en marcha desde el exterior de la cámara apantallada mediante el switch externo y con un solo magnetrón en funcionamiento, por lo tanto $900 \mathrm{~W}$ de potencia y polarización lineal; mientras, un ordenador (también desde el exterior) monitoriza la temperatura de la muestra (tabla o tabla con larvas) mediante sensores ópticos de temperatura, es importante acondicionar la mesa para lograr que la punta de los sensores ópticos no sufra daños por una flexión excesiva. El espacio de aire existente entre la muestra y el aplicador es de $2 \mathrm{~cm}$, y el de la mesa con la muestra es de $1 \mathrm{~cm}$; estos espacios se logran colocando tacos de teflón en medio, y existen con el fin de evitar condensación en la superficie de la madera.

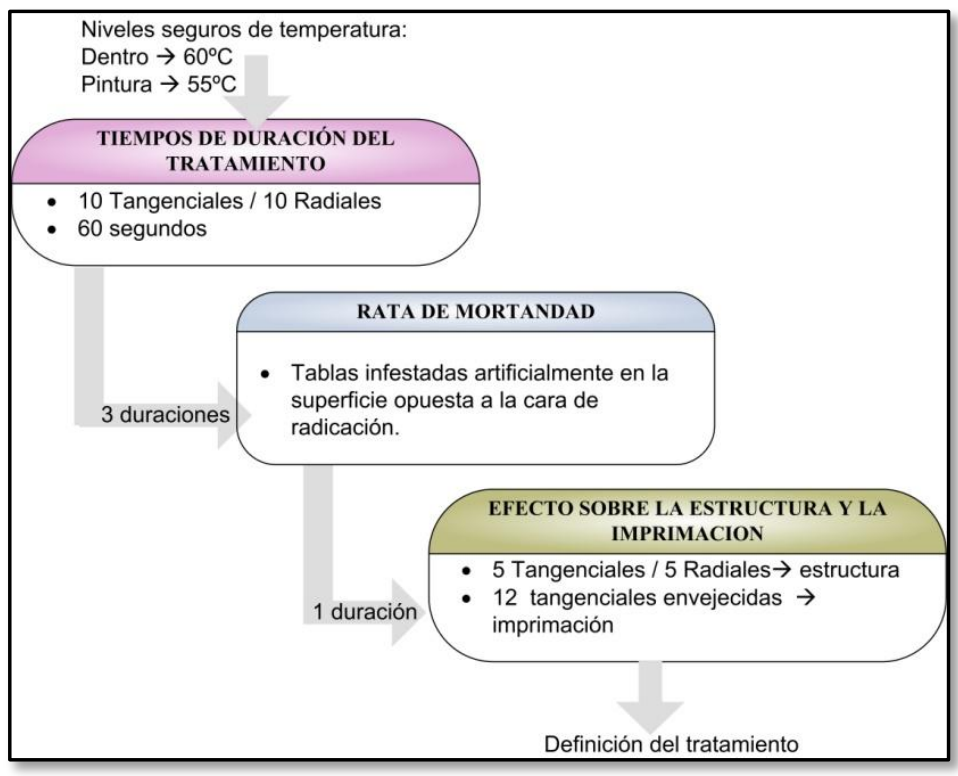

Figura 3-2. Fases de la Experimentación 
La experimentación está dividida en tres fases (Figura 3-2) relacionadas con los criterios a tener en cuenta para conseguir el objetivo. Cada fase da los resultados necesarios para seguir con la siguiente y aunque todas tienen la misma configuración, varía la preparación de la muestra, la disposición de las sondas ópticas, el número de muestras tratadas y el equipamiento adicional.

La primera fase busca determinar la duración en la que la temperatura alcanzada por la madera no genera daños estructurales que puedan afectar el interior o la cara pintada de la misma. Los límites de seguridad se fijan en $60{ }^{\circ} \mathrm{C}$ para el interior y $55^{\circ} \mathrm{C}$ para la superficie. Para conseguir estos tiempos se tratan cinco tablas radiales y cinco tangenciales, ubicando el campo eléctrico paralelo a las fibras de la madera en la cara expuesta directamente a la radiación (cara de radiación), y luego el mismo número de muestras con las misma características se tratan con el campo eléctrico en posición perpendicular a las fibras de la cara de radiación, esto se logra rotando el aplicador $90^{\circ}$ en dirección a las manecillas del reloj.

Las 20 muestras se radian durante 60 segundos. En este tiempo la temperatura se mide en cuatro puntos (Figura 3-3):

1. Cara de radiación: la sonda es colocada superficialmente en el centro de la tabla.

2. Cara opuesta: la sonda se ubica superficialmente en el centro de la cara opuesta a la cara de radiación. Junto con la sonda anterior permiten identificar qué cara se calienta más, siendo ésta la máxima temperatura que se puede alcanzar en la cara pintada.

3. Interior centro: la sonda se introduce $1,5 \mathrm{~cm}$ en el centro de la madera; identifica la máxima temperatura que se puede alcanzar dentro de la madera.

4. Interior paralela: la sonda se introduce $1,5 \mathrm{~cm}$ en la madera a $8 \mathrm{~cm}$ de distancia desde el centro de la madera en la dirección paralela a las fibras de la cara de radiación. Mide la atenuación de la temperatura dentro del área del aplicador del sistema. Se ha escogido esta dirección porque el calentamiento es mayor debido a que las propiedades dieléctricas en esta dirección son mayores que las de la dirección perpendicular a las fibras.

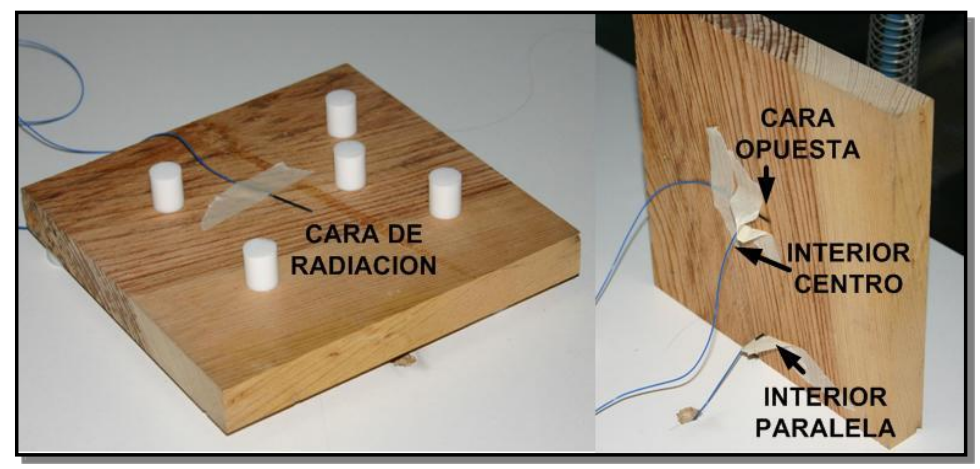

Figura 3-3. Ubicación de la sondas de temperatura (1ra fase) 
Las consideraciones para la selección de los tres tratamientos que se usan en la fase dos se encuentran en la Tabla 3-2, es decir, se seleccionan tres tiempos de acuerdo a la temperatura interna, aquellos que no superen $\operatorname{los} 60^{\circ} \mathrm{C}$ teniendo en cuenta la desviación estándar una, dos o tres veces, y luego se verifica que la temperatura superficial en los tiempos seleccionados y sumándole dos veces la desviación estándar permanezca por debajo de $55^{\circ} \mathrm{C}$.

Tabla 3-2. Consideraciones para la selección de los tratamientos

\begin{tabular}{|c|c|c|c|}
\hline $\begin{array}{l}\text { Duración } \\
\text { (s) }\end{array}$ & $\begin{array}{l}\text { Temperatura Interna } \\
{ }^{\circ} \mathrm{C}\end{array}$ & \multirow{4}{*}{$\&$} & $\begin{array}{l}\text { Temperatura Superficial } \\
{ }^{\circ} \mathrm{C}\end{array}$ \\
\hline $\mathrm{T}_{1}$ & $\operatorname{Max} t\left[\mathrm{~T}_{\text {interna }}(\mathrm{t})+\sigma(\mathrm{t}) \leq 60^{\circ} \mathrm{C}\right]$ & & $\operatorname{SI}\left[\mathrm{T}_{\text {superficie }}\left(\mathrm{t}_{1}\right)+2 \sigma\left(\mathrm{t}_{1}\right) \leq 55^{\circ} \mathrm{C}\right]$ \\
\hline $\mathrm{T}_{2}$ & $\operatorname{Max} \mathrm{t}\left[\mathrm{T}_{\text {interna }}(\mathrm{t})+2 \sigma(\mathrm{t}) \leq 60^{\circ} \mathrm{C}\right]$ & & $\mathrm{SI}\left[\mathrm{T}_{\text {superficie }}\left(\mathrm{t}_{2}\right)+2 \sigma\left(\mathrm{t}_{2}\right) \leq 55^{\circ} \mathrm{C}\right]$ \\
\hline $\mathrm{T}_{3}$ & $\operatorname{Max} t\left[\mathrm{~T}_{\text {interna }}(\mathrm{t})+3 \sigma(\mathrm{t}) \leq 60^{\circ} \mathrm{C}\right]$ & & $\mathrm{SI}\left[\mathrm{T}_{\text {superficie }}\left(\mathrm{t}_{3}\right)+2 \sigma\left(\mathrm{t}_{3}\right) \leq 55^{\circ} \mathrm{C}\right]$ \\
\hline
\end{tabular}

En la segunda fase el objetivo es evaluar la tasa de mortalidad de larvas y huevos infestados artificialmente en tablas con los tratamientos encontrados en la fase uno. Para este fin, seis larvas son colocadas por pares en tres diferentes localizaciones (Figura 3-4) sobre la cara opuesta de la tabla. Se usan siete tablas: tres radiales y cuatro tangenciales (en total se tratan cuarenta y dos larvas).

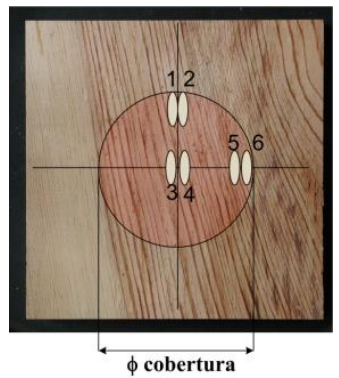

Figura 3-4. Localización larvas

La población de control está compuesta por doce larvas, que son sometidas al mismo estrés que grupo tratado, es decir, se extraen de la madera original para colocarlas en huecos individuales sobre la superficie de otra pieza de madera (Figura 3-5). Luego se transportan desde la zona de conservación hasta la zona de experimentación y después de la experimentación se devuelven a la zona de conservación, donde son observadas. Los valores obtenidos de mortalidad se consideran validos si el $70 \%$ de la población de control se encuentra en perfecto estado una vez concluida la observación. Para la radiación se usa una aplicación central continua, es decir que durante el tiempo de tratamiento se hace coincidir el centro del aplicador y el de las tablas. 


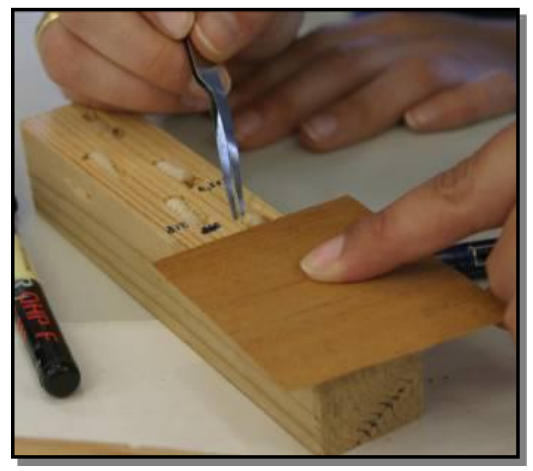

Figura 3-5. Manipulación de larvas

La tercera fase examina el efecto del tratamiento seleccionado en la segunda fase, sobre la estructura de la madera y la imprimación. En el primer caso, cinco tablas tangenciales se tratan durante el tiempo seleccionado en la fase dos. Para determinar los movimientos de la madera, se mide el espesor de la tabla antes y después del tratamiento mediante un comprador centesimal. Los puntos medidos se muestran en la matriz de la Figura 3-6. Las líneas continuas de la figura atraviesan la tabla por el centro (a $10 \mathrm{~cm}$ del borde) y las discontinuas están separadas $2 \mathrm{~cm}$ del borde de la tabla.

El centro de la radiación se localiza en la mitad de uno de los cuadrantes, ya que de producirse deformaciones en la madera las esquinas serían los puntos más sensibles. También se localizan cuatro sondas de temperatura (puntos azules Figura 3-6):

1. paralelo a las fibras desde el centro de la radiación,

2. diagonal al centro de la radiación,

3. centro de la radiación y

4. perpendicular a las fibras desde el centro de la radiación.

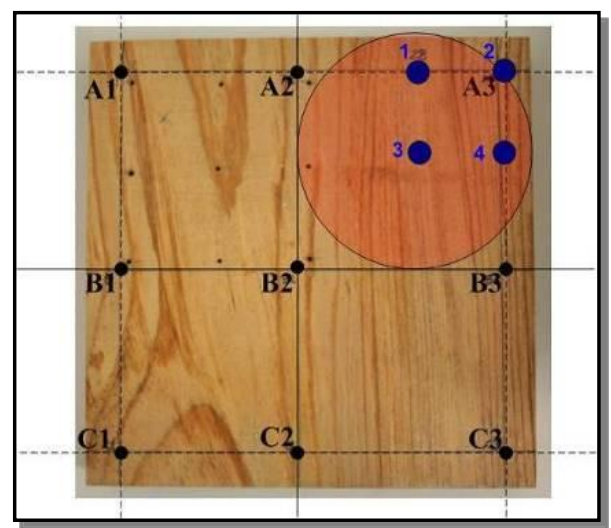

Figura 3-6. Matriz para la medida de movimientos. 
En el segundo caso, se envejecen en una cámara de envejecimiento doce tablas tangenciales, preparadas en varios grupos según se indica en la Tabla 3-3. Las tablas sin imprimación se envejecen con el objetivo de comparar los resultados de temperatura con los obtenidos en la fase uno y comprobar que sobre la imprimación no se superan los $55^{\circ} \mathrm{C}$ de temperatura.

Tabla 3-3. Tablas preparadas con imprimación

\begin{tabular}{||c|c|c||}
\hline \hline NO. & ITEM & SUBTRATO \\
\hline 4 & Sin imprimación & --- \\
\hline 2 & Con imprimación & --- \\
\hline 4 & Con imprimación & Tela \\
\hline 2 & Con imprimación & Estopa \\
\hline
\end{tabular}

Luego, la mitad de las muestras de cada grupo se radian por la cara sin imprimación (R1) y la otra mitad por la cara con imprimación (R2), durante el tratamiento la temperatura se mide en tres puntos ubicados en el centro de la radiación:

1. La cara de radiación.

2. La cara opuesta.

3. El interior.

Después del tratamiento se hace con una cámara termográfica una termografía de la cara de radiación, la cara opuesta y las caras internas de la muestra. Las tablas han sido cortadas previamente al tratamiento y durante el mismo se mantienen unidas con cinta adhesiva de papel. En la Figura 3-7 se indica:

- La zona de aplicación del tratamiento (zona roja en la parte izquierda de la figura).

- Localización de las sondas ópticas, en el centro de la zona irradiada.

- Disposición de la tabla cortada (las líneas punteadas indican los cortes).

- Disposición de las caras para la termografía (en la parte izquierda de la Figura 3-7 se indican las caras y en la derecha como se organizan para la termografía). 


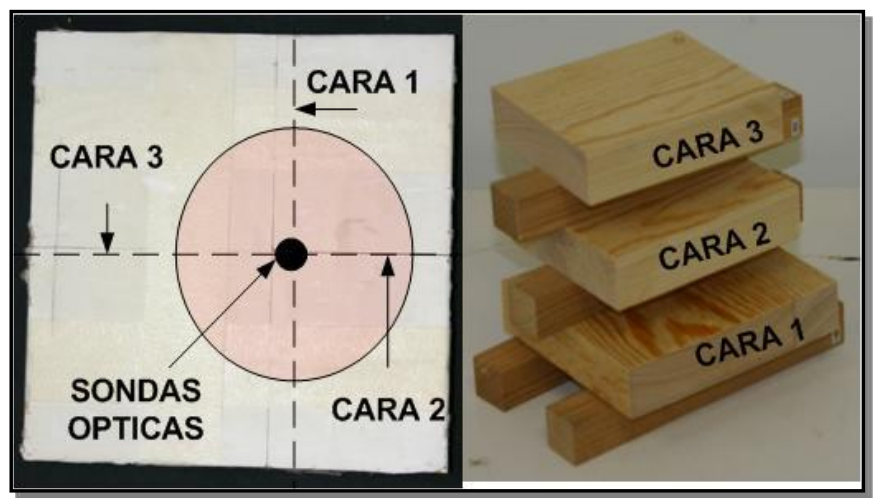

Figura 3-7. Configuración para termografías

Adicionalmente, se hacen pruebas de temperatura con listones y tablas con nudos, radiándolas en ambos casos con el campo eléctrico paralelo a las fibras de la cara de radicación. En el primer caso se utilizan diez listones de dimensiones 5x5x10 cm y se les mide la temperatura durante un tratamiento de 50 segundos. Para los primeros cinco se ubican las sondas internamente a las siguientes profundidades: 0,$5 ; 1,8 ; 3,2$ y 4,5 cm desde la cara de radiación (Figura 3-8).

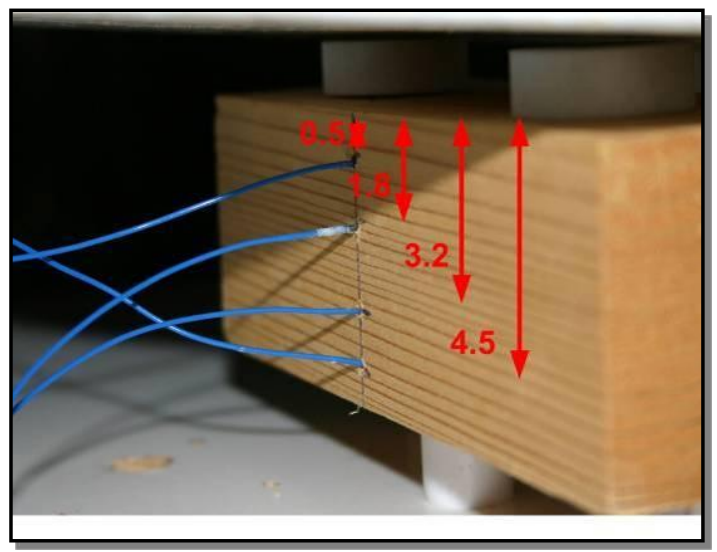

Figura 3-8.Configuración para temperatura interior en listones

A los listones restantes se les mide la temperatura superficial en las cuatro caras de mayor área, para lo cual las sondas se colocan en contacto con la superficie en el centro de la cara como se indica en el esquema de la Figura 3-9, radiándose el listón por la cara 1. Las sondas son identificadas según la numeración, como sigue:

1. Lado superior

2. Lado izquierdo

3. Lado posterior

4. Lado derecho 


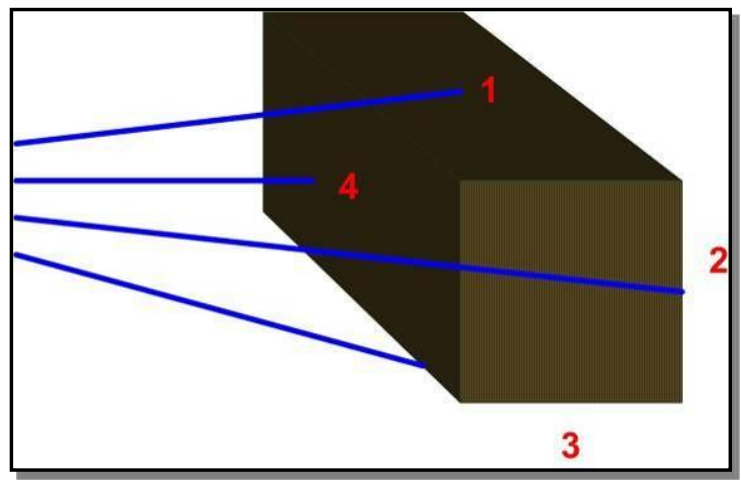

Figura 3-9.Configuración para temperatura superficial en listones

En relación con los nudos, se escogen dos tablas tangenciales que tengan nudos grandes y visibles (Figura 3-10). Luego se radian durante 25 segundos con el centro de la antena sobre el nudo y éste en la cara opuesta a la radiación. Durante el tratamiento se mide la temperatura sobre el nudo con el fin de verificar que no se alcanzan los niveles de seguridad propuestos y los valores de temperatura no superan las temperaturas conseguidas en las etapas anteriores.

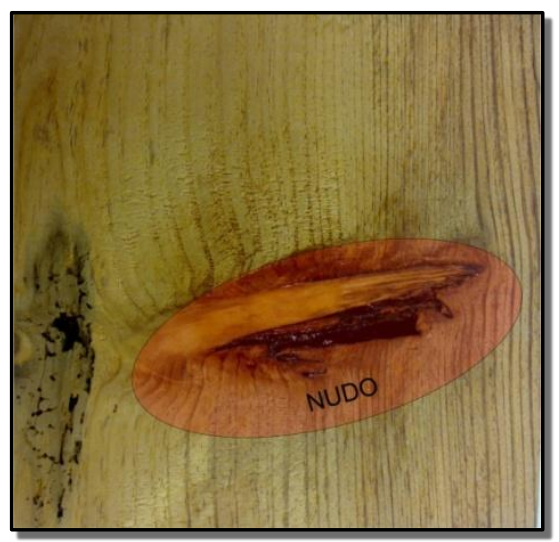

Figura 3-10. Nudo en tabla tratada

\subsection{TIEMPO DE DURACIÓN DEL TRATAMIENTO}

El primer objetivo es determinar tres tiempos en los cuales los niveles de temperatura estén por debajo de los límites escogidos; para ello se aplica un tratamiento de $900 \mathrm{~W}$ durante 60 segundos, midiendo la temperatura en los cuatro puntos de la Figura 3-3 para cuatro casos diferentes, dependiendo del tipo de muestra y de la orientación del campo:

A. Campo eléctrico paralelo a la fibras en la cara de radiación, aplicado a tablas tangenciales.

B. Campo eléctrico paralelo a las fibras en la cara de radiación, aplicado a tablas radiales. 
C. Campo eléctrico perpendicular a la fibras en la cara de radiación, aplicado a tablas tangenciales.

D. Campo eléctrico perpendicular a las fibras en la cara de radiación, aplicado a tablas radiales.

Los resultados de temperatura ${ }^{1}$ se muestran en la Figura 3-11 y donde se observa que, como era de esperar, la temperatura en el interior de la madera (Interior centro) es superior a los otros tres puntos controlados, mientras que el valor de la temperatura en las dos superficies (cara de radiación y cara opuesta) es similar, aunque en la cara opuesta es ligeramente superior.

También puede observarse que a $8 \mathrm{~cm}$ desde el centro de la muestra (Interior paralela) no hay ninguna variación de temperatura durante la radiación con microondas, lo que significa que el área de acción del aplicador sobre la madera es inferior a su área física $(\phi 20 \mathrm{~cm})$.

Por otro lado, la temperatura es mayor cuando el campo eléctrico es paralelo a las fibras, tanto para nuestras radiales como para muestras tangenciales. Adicionalmente, y cuando la orientación del campo es la misma, la temperatura es mayor para muestras tangenciales. Por lo tanto, el caso más restrictivo es la muestra tangencial radiada con el campo paralelo a las fibras (caso A).

Evaluando la temperatura en los puntos interior centro y cara opuesta para el caso más restrictivo, los tiempos de tratamiento seleccionados según las restricciones de la Tabla 3-2 son: 14,4; 12 y 9,6 segundos, estos datos se muestran en la Tabla 3-4.

Tabla 3-4. Tiempos para tratamiento inicial

\begin{tabular}{||c|r|r|r|r|r||}
\hline \multirow{3}{*}{} & \multicolumn{5}{|c||}{ T a n g e n c i a l } \\
\cline { 2 - 6 } & \multicolumn{4}{|c|}{ Interior centro } & \multicolumn{2}{c||}{ Cara posterior } \\
\cline { 2 - 6 } & $\begin{array}{c}\mathbf{T} \\
(\mathbf{s})\end{array}$ & $\begin{array}{c}\text { Mediana } \\
{ }^{\circ} \mathrm{C}\end{array}$ & $\begin{array}{c}\text { T. limite } \\
{ }^{\mathbf{0}} \mathbf{C}\end{array}$ & $\begin{array}{c}\mathrm{T} . \\
{ }^{\circ} \mathrm{C}\end{array}$ & $\mathbf{2} \sigma$ \\
\hline $1 \sigma$ & 14,4 & 49,3 & 56,71 & 34,8 & 48,03 \\
\hline $2 \sigma$ & 12 & 44,24 & 57,40 & 32,08 & 43,05 \\
\hline $3 \sigma$ & 9,6 & 39,1 & 55,85 & 29,02 & 37,52 \\
\hline
\end{tabular}

${ }^{1}$ Mediana de las 5 muestras tratadas para cada caso.

$\overline{50}$ Tecnología de microondas en el sector de restauración de bienes culturales 


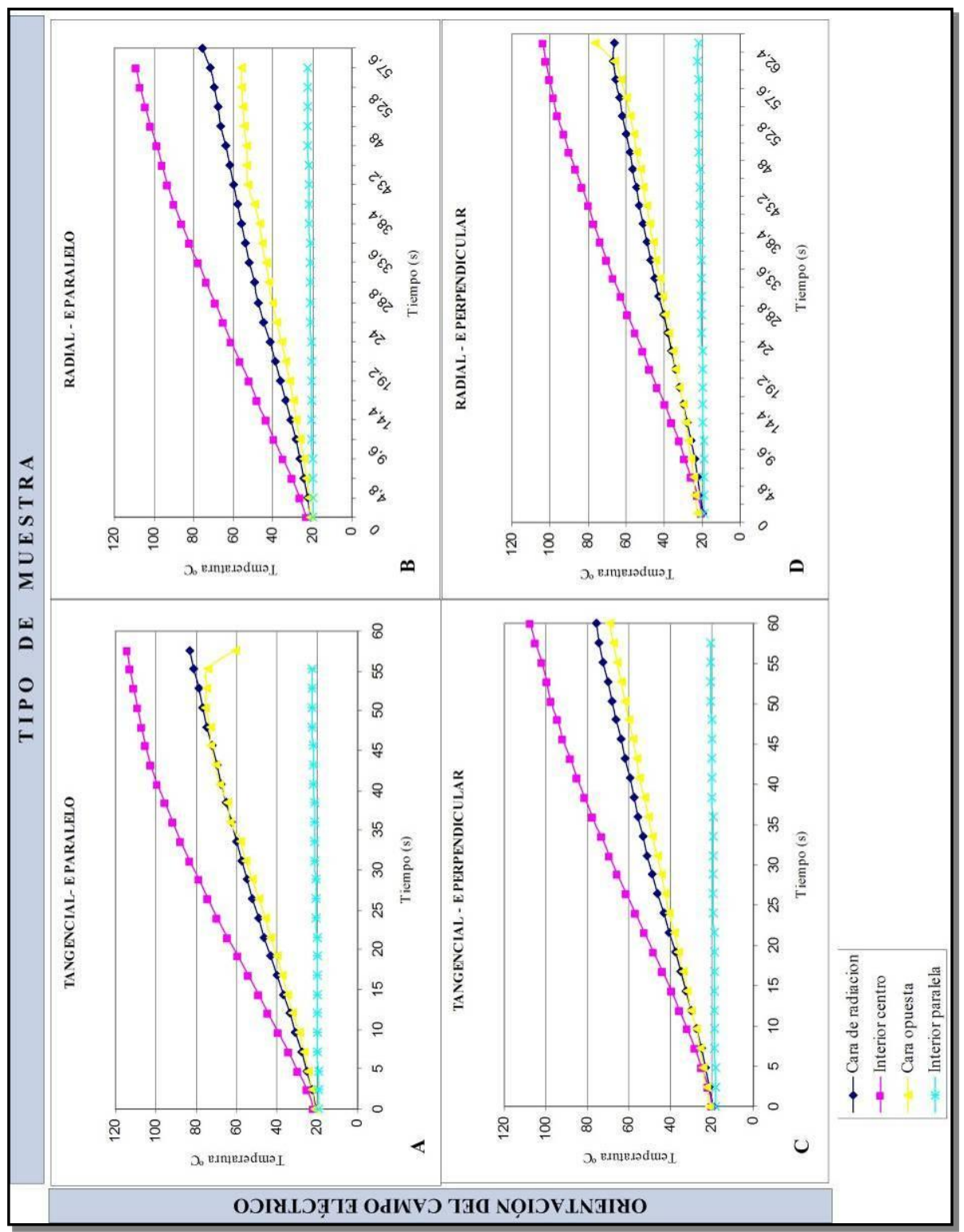

Figura 3-11. Medidas de Temperatura inicial

En contraposición, se tiene que el índice de dispersión con respecto a la mediana [43] del punto cara opuesta para el caso A, supera el $20 \%$ para tiempos superiores a 14,4 segundos, valor de dispersión que no es alcanzado por ninguno de los otros casos o puntos evaluados (Apéndice II. i). Esto es importante ya que la mediana no sería un valor representativo para la temperatura alcanzada por la madera para 
tiempos superiores a 14,4 segundos, por lo que la generalización de un tratamiento sería muy arriesgada.

Por el motivo expuesto en el párrafo anterior, se plantea repetir el experimento con las mismas muestras, prestando especial atención a la colocación de las sondas en la madera. Los objetivos son determinar el área de acción del aplicador sobre la madera ubicando el punto interior paralelo dentro de ésta y reducir el índice de dispersión encontrado en el punto cara opuesta dentro del caso de interés (caso A).

Se espera obtener un resultado diferente en la cara posterior, ya que el espacio de aire entre la muestra y la mesa en el experimento anterior fue de $1 \mathrm{~cm}$ y en la repetición se amplía a $2 \mathrm{~cm}$, la misma distancia que hay entre el aplicador y la muestra. Esto permite una circulación de aire en las mismas condiciones que en la cara de radiación.

Para determinar el área de acción del aplicador, se tiene en cuenta el módulo del campo eléctrico del aplicador, que consiste en una guía cilíndrica $\mathrm{TE}_{11}$.

En la Figura 3-12 se muestra el módulo del campo eléctrico normalizado, donde el círculo de $10 \mathrm{~cm}$ de diámetro, radica el área en la que el módulo del campo eléctrico es mayor a 0,5, demostrando que el decaimiento del campo es significativo a partir de $5 \mathrm{~cm}$ medidos desde el centro del aplicador. En consecuencia, la distancia desde el centro de la muestra en dirección paralela a las fibras se reduce a $4 \mathrm{~cm}$, para las nuevas medidas de temperatura.

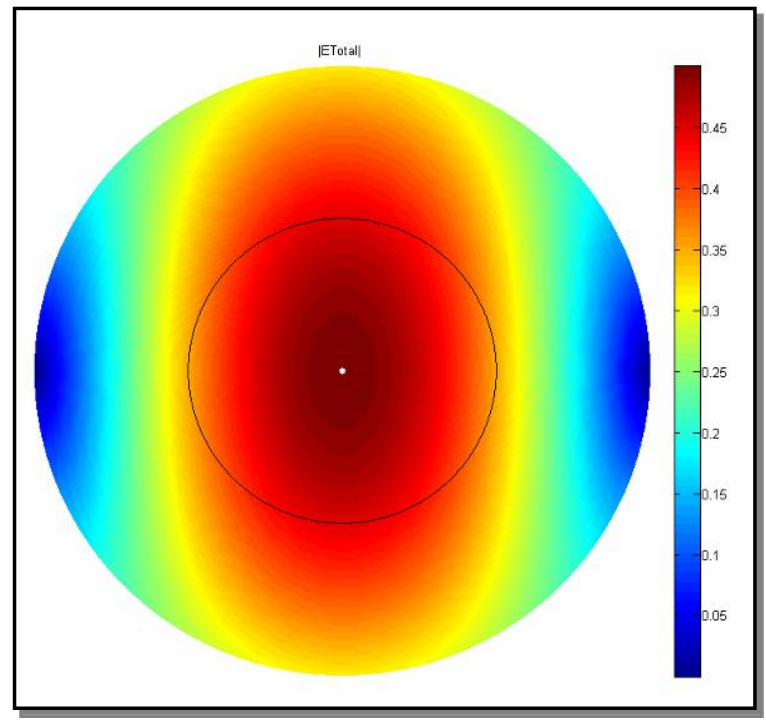

Figura 3-12. Factor de forma del campo eléctrico del aplicador

Para obtener la Figura 3-12 se calcula el módulo del campo eléctrico y se representa gráficamente. El campo eléctrico total de una guía cilíndrica $\mathrm{TE}_{11}$ [44] está dado por: 


$$
\left|E_{T}\right|^{2}=\left|E_{\rho}\right|^{2}+\left|E_{\varphi}\right|^{2}
$$

Teniendo en cuenta que:

$$
\begin{gathered}
E_{\varphi}=Z_{T E}\left(H_{\rho} \hat{\rho} \times \hat{z}\right)=Z_{T E} H_{\rho} \hat{\varphi} \\
E_{\rho}=Z_{T E}\left(H_{\varphi} \hat{\varphi} \times \hat{z}\right)=Z_{T E} H_{\varphi} \hat{\rho} \\
H_{\rho}=-\frac{\gamma}{k_{c}} H_{M} J_{m}^{\prime}\left(k_{c} \rho\right) \cos (\varphi) \\
H_{\varphi}=\frac{\gamma}{k_{c}^{2}} \frac{m}{\rho} H_{M} J_{m}\left(k_{c} \rho\right) \sin (\varphi) \\
\gamma=\beta \quad y \quad m=1 \\
\frac{d}{d x} J_{n}\left(A_{x}\right)=A J_{n-1}\left(A_{x}\right)-\frac{n}{x} J_{n}\left(A_{x}\right)
\end{gathered}
$$

El campo total es:

$$
\begin{aligned}
& E_{\varphi} \\
& =\frac{Z_{T E} \beta}{k_{C}} H_{M} \sqrt{\left(\left[J_{0}\left(k_{C} \rho\right)-\frac{J_{1}\left(k_{C} \rho\right)}{k_{C} \rho}\right] \cos ^{2}(\varphi)+\frac{J_{1}\left(k_{C} \rho\right)^{2}}{k_{C} \rho} \sin ^{2}(\varphi)\right)}
\end{aligned}
$$

En la Figura 3-12 se representa el factor de forma que es lo que se encuentra en la raíz, con el argumento $k_{C} \rho$ variando de 0 hasta 1,8412 .

Los resultados de temperatura del nuevo experimento se muestran en la Figura 3-13. Las diferencias respecto a las medidas de la Figura 3-11 son que el punto interior paralelo está a $4 \mathrm{~cm}$ desde el centro y que la distancian entre la muestra y la mesa es de $2 \mathrm{~cm}$.

Los resultados confirman que el calentamiento es más rápido en el interior de la madera, y muestran que la temperatura en el punto cara posterior es mayor que la de la cara de radiación, por lo que sigue siendo válido utilizar los puntos cara posterior e interior centro como referencia para seleccionar los tiempos del tratamiento.

En relación con el caso más restrictivo, se tiene que para el mismo tipo de muestra se calientan más rápidamente cuando la radiación es paralela a las fibras, y en este caso la muestra que alcanza mayor temperatura durante el tratamiento es la muestra tangencial (caso A). Utilizando la Tabla 3-2, los tiempos de tratamiento están entre 14 y 20 segundos, mientras que sin tener en cuenta la desviación estándar del valor de temperatura este rango se amplía hasta 24 segundos (Tabla 3-5). 
En el Apéndice II.i puede verse que la desviación estándar para el caso A es mayor de $20 \%$ sólo para tiempos superiores a 40 segundos, validando las medidas y suposiciones en el rango de interés.

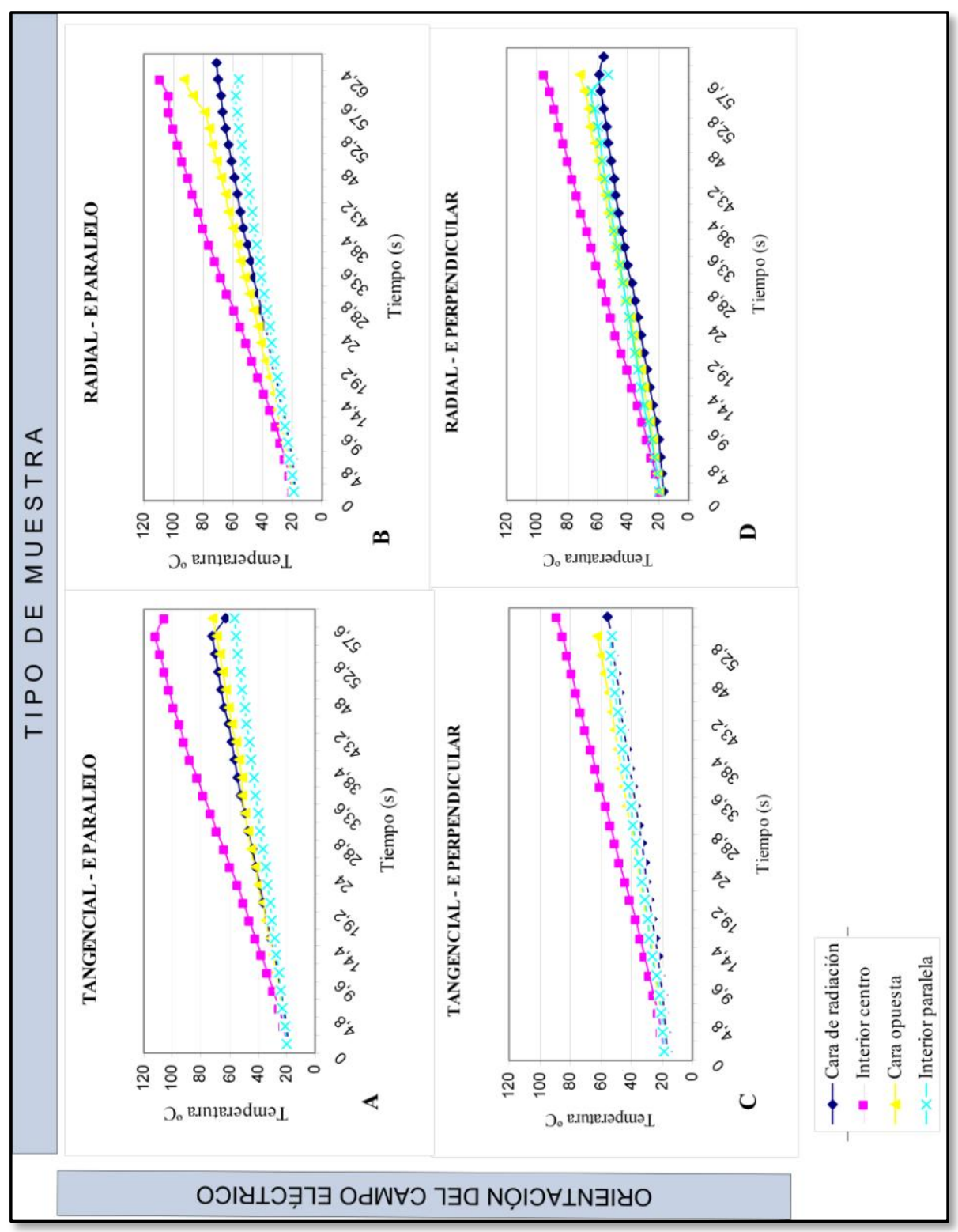

Figura 3-13. Medidas de temperatura 
Tabla 3-5. Tiempos de tratamiento

\begin{tabular}{||c|r|r|r|r|r||}
\hline \multirow{3}{*}{} & \multicolumn{5}{|c|}{ T a n g e n c i a l } \\
\cline { 2 - 6 } & \multicolumn{4}{|c|}{ Centro Interna } & \multicolumn{2}{c|}{ Centro posterior } \\
\cline { 2 - 6 } & $\begin{array}{c}\mathbf{t} \\
\mathbf{( s )}\end{array}$ & $\begin{array}{c}\text { Mediana } \\
{ }^{\circ} \mathrm{C}\end{array}$ & $\begin{array}{c}\text { T. limite } \\
{ }^{\mathbf{0}} \mathbf{C}\end{array}$ & $\begin{array}{c}\mathrm{T} \\
{ }^{\circ} \mathrm{C}\end{array}$ & $\mathbf{2} \boldsymbol{\sigma}$ \\
\hline $0 \sigma$ & 24 & 59,02 & 59,02 & 41,42 & 55,98 \\
\hline $1 \sigma$ & 19,2 & 52,32 & 56,52 & 36,78 & 48,42 \\
\hline $2 \sigma$ & 16,8 & 45,96 & 56,93 & 34,08 & 44,48 \\
\hline $3 \sigma$ & 14,4 & 42,00 & 56,52 & 31,48 & 40,57 \\
\hline
\end{tabular}

Teniendo en cuenta todos los resultados anteriormente discutidos, el rango, de donde se seleccionan los tiempos de tratamiento para la siguiente fase, es el comprendido entre 10 y 25 segundos, ya que en el límite superior aun se encuentra bajo el nivel de seguridad requerido, aunque probablemente no todas las muestras se queden por debajo del límite. Así pues, los tiempos seleccionados son: 15, 20 y 25 segundos.

\subsection{TASA DE MORTALIDAD}

Los resultados de tasa de mortalidad en relación al número de larvas tratadas se muestran en la Tabla 3-6. En la experimentación la radiación es central ${ }^{2}$, el campo eléctrico está orientado paralelo a la fibras, las larvas están ubicadas según la Figura 3-4 y los tiempos de radiación son 15, 20 y 25 segundos.

En los tres casos puede observarse que la efectividad del tratamiento en el punto central es del $100 \%$, mientras en los puntos paralelo y perpendicular no supera el $50 \%$; sin embargo este último porcentaje tiene tendencia a disminuir puesto que sólo se trataron dos larvas en algunos casos. Adicionalmente, la muerte de las larvas en el punto central es inmediata, mientras en los otros dos puntos se produce transcurrido cierto tiempo de observación. La validez de los resultados se confirma ya que la población de control continúa en buen estado (los datos de la observación constan en el Apéndice II. ii).

En resumen se tiene una efectividad baja, ya que la tasa de mortalidad inmediata del tratamiento central es del $28,57 \%$ y transcurrido un tiempo de observación de 70 días aumenta al 52,38\%. Debido a la poca efectividad en los puntos paralelo y perpendicular se plantea la posibilidad de un efecto acumulado sobre las larvas, sometiéndolas a radiaciones solapadas sólo en dichas áreas. Estas radiaciones deben ser de iguales características ( $900 \mathrm{~W}$ y tiempo fijo) y espaciadas un intervalo de tiempo.

${ }^{2}$ Haciendo coincidir el centro de la muestra y del aplicador. 
Tabla 3-6. Tasa de mortalidad con aplicación central

\begin{tabular}{|c|c|c|c|c|c|c||}
\hline \multirow{2}{*}{ LOCALIZACIÓN } & \multicolumn{2}{|c|}{ Tratamiento 15 s } & \multicolumn{2}{c|}{ Tratamiento 20 s } & \multicolumn{2}{c|}{ Tratamiento 25 s } \\
\cline { 2 - 7 } & $\begin{array}{c}\text { No. } \\
\text { Larvas }\end{array}$ & T.M. $^{3}$ & $\begin{array}{c}\text { No. } \\
\text { Larvas }\end{array}$ & $\begin{array}{c}\text { T.M. } \\
\begin{array}{c}\text { No. } \\
\text { Larvas }\end{array}\end{array}$ & T.M. \\
\hline \multirow{2}{*}{ Centro } & 4 & $\begin{array}{c}100 \% \\
4 / 4\end{array}$ & 8 & $\begin{array}{c}100 \% \\
8 / 8\end{array}$ & 2 & $\begin{array}{c}100 \% \\
2 / 2\end{array}$ \\
\hline 4 cm paralelo & 4 & $0 \%$ & 8 & $25 \%$ & 2 & $\begin{array}{c}50 \% \\
1 / 2\end{array}$ \\
\hline \multirow{2}{*}{4 cm perpendicular } & 2 & $50 \%$ & 8 & $37,5 \%$ & 2 & $50 \%$ \\
& & $1 / 2$ & $8 / 8$ & & $1 / 2$ \\
\hline
\end{tabular}

Para determinar la duración de cada radiación en el solapamiento, se tiene en cuenta que con el tratamiento de 15 segundos no muere ninguna larva en el punto paralelo, por lo que la elección está entre los tratamiento de 20 y 25 segundos; ya que con el tratamiento de 25 segundos, no se puede afirmar una mejora significativa en la efectividad y en cambio sí se trabajaría cerca de los límites de temperatura de la madera, el tiempo de tratamiento seleccionado es de 20 segundos.

\subsubsection{Modelos para radiaciones solapadas}

Los modelos propuestos para solapar las radiaciones en las áreas problemáticas se muestran en la Figura 3-14, estos modelos varían según la disposición de los centros y su separación:

a. Separación entre centros de $7 \mathrm{~cm}$ y disposición cuadrada.

b. Separación entre centros de $6 \mathrm{~cm}$ y disposición cuadrada.

c. Separación entre centros de $7 \mathrm{~cm}$ y disposición triangular isósceles.

d. Separación entre centros de $7 \mathrm{~cm}$ y disposición triangular equilátera.

Con un tiempo de radiación de 20 segundos y un intervalo entre radiaciones de 25 segundos, el tiempo requerido por los modelos (a) y (b) para cubrir áreas cuadradas esta dado por:

$$
\begin{gathered}
N=\frac{\left(L_{x}+S_{x}\right)}{m_{x}} \\
t_{\text {fila }}=(N \times 20 s)+((N-1) \times 25 s) \\
t_{\text {total }}=\left(t_{\text {fila }} \times N\right)+((N-1) \times 25 s)
\end{gathered}
$$

\footnotetext{
${ }^{3}$ Tasa de mortalidad
} 


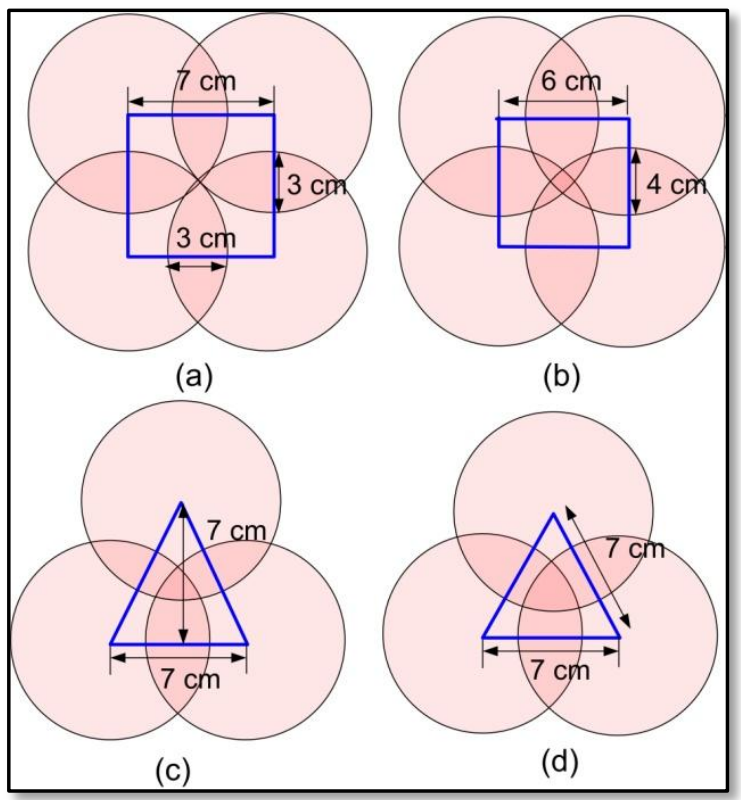

Figura 3-14. Modelos para solapar radicaciones

El subíndice x determina el modelo, donde:

L Lado del área cuadrada que se quiere cubrir en centímetros $\mathrm{x}=$ (a) Debe ser un múltiplo de $7 \mathrm{~cm}$ y restarle $3 \mathrm{~cm}$. $\mathrm{x}=$ (b) Debe ser múltiplo de $6 \mathrm{~cm}$ y restarle $4 \mathrm{~cm}$.

S Solapamiento en la dirección de aplicación $\mathrm{x}=(\mathrm{a})$ es $3 \mathrm{~cm} \quad \mathrm{x}=(\mathrm{b})$ es $4 \mathrm{c} \mathrm{m}$

m Factor de cubrimiento

$$
\mathrm{x}=(\mathrm{a}) \text { es } 7 \mathrm{~cm} \quad \mathrm{x}=(\mathrm{b}) \text { es } 6 \mathrm{~cm}
$$

$\mathrm{N}$ número lineal de aplicaciones

$\mathrm{t}_{\text {fila }} \quad$ tiempo para cubrir una distancia lineal

$\mathrm{t}_{\text {total }} \quad$ tiempo total para cubrir un área cuadrada

El tiempo requerido por el modelo (c) bajo los mismos supuestos es:

$$
\begin{gathered}
N=\frac{\left(L_{x}+S_{x}\right)}{m_{x}} \\
t_{\text {fila } A}=(N \times 20 s)+((N-1) \times 25 s)
\end{gathered}
$$




$$
\begin{gathered}
t_{\text {fila } B}=((N+1) \times 20 s)+(N \times 25 s) \\
t_{\text {total }}=\left(t_{\text {fila } A} \times A\right)+\left(t_{\text {fila } B} \times B\right)+((N-1) \times 25 s)
\end{gathered}
$$

Donde:

L, S, m Tienen el mismo valor que en el caso (a).

$\mathrm{t}_{\text {fila A }} \quad$ Tiempo requerido para cubrir una distancia lineal en filas impares.

$\mathrm{t}_{\text {fila B }} \quad$ Tiempo requerido para cubrir una distancia lineal en filas pares.

A N/2 redondeado al próximo entero.

B N/2 redondeado al anterior entero.

Finalmente, el tiempo requerido por el modelo (d) bajo los mismos supuestos para cubrir áreas rectangulares con el mismo número de radiaciones que el modelo (c) es:

$$
\begin{gathered}
N_{x}=\frac{\left(L_{x}+S_{x}\right)}{m_{x}} \\
N_{y}=\frac{\left(L_{y}+S_{y}\right)}{m_{y}} \\
t_{\text {fila } A}=\left(N_{x} \times 20 s\right)+\left(\left(N_{x}-1\right) \times 25 s\right) \\
t_{\text {fila } B}=\left(\left(N_{x}+1\right) \times 20 s\right)+\left(N_{x} \times 25 s\right) \\
t_{\text {total }}=\left(t_{\text {fila } A} \times A\right)+\left(t_{\text {fila } B} \times B\right)+\left(\left(N_{y}-1\right) \times 25 s\right)
\end{gathered}
$$

Donde:

L Lado del área rectangular que se quiere cubrir en centímetros en direcciones $x$ y $y$.

$\mathrm{x}=$ Debe ser un múltiplo de $7 \mathrm{~cm}$ y restarle $3 \mathrm{~cm}$

y= Debe ser múltiplo de $6,062 \mathrm{~cm}$ y restarle $3,938 \mathrm{~cm}$.

S Solapamiento en la dirección de aplicación

$\mathrm{x}=\mathrm{es} 3 \mathrm{~cm} \quad \mathrm{y}=\mathrm{es} 3,938 \mathrm{~cm}$

m Factor de cubrimiento

$$
\mathrm{x}=\mathrm{es} 7 \mathrm{~cm} \quad \mathrm{y}=\mathrm{es} 6,062 \mathrm{~cm}
$$

$\mathrm{t}_{\text {fila A }} \quad$ Tiempo requerido para cubrir una distancia lineal en filas impares.

$\mathrm{t}_{\text {fila B }} \quad$ Tiempo requerido para cubrir una distancia lineal en filas pares. 

A $\quad \mathrm{N}_{\mathrm{y}} / 2$ redondeado al próximo entero.
B $\quad \mathrm{N}_{\mathrm{y}} / 2$ redondeado al anterior entero.

De este modo para cubrir un área de un metro cuadrado aproximadamente, el tiempo requerido para cada uno de los modelos se muestra en la Tabla 3-7. Puede observarse que para cubrir dicha área el modelo (b) requiere un $33 \%$ más de tiempo que los demás, los cuales utilizan alrededor de 3 horas.

Tabla 3-7. Tiempos empleados en modelos con solape

\begin{tabular}{||c|c|c|}
\hline MODELO & AREA & TIEMPO \\
\hline A & $102 \times 102 \mathrm{~cm}^{2}$ & 2 horas y 48 minutos \\
\hline B & $104 \times 104 \mathrm{~cm}^{2}$ & 4 horas y 3 minutos \\
\hline C & $102 \times 102 \mathrm{~cm}^{2}$ & 2 horas y 54 minutos \\
\hline D & $102 \times 99,116 \mathrm{~cm}^{2}$ & 3 horas y 17 minutos \\
\hline
\end{tabular}

Definiendo la eficiencia de cada modelo como el área de la zona tratada sobre el área total de las radiaciones aplicadas, en la Tabla 3-8 se muestra una comparativa entre modelos (planos en Apéndice III).

Tabla 3-8. Comparativa modelos con solape

\begin{tabular}{|c|c|c|c|c|c|}
\hline & & $\mathbf{a}$ & b & c & d \\
\hline \multicolumn{2}{|c|}{$\begin{array}{l}\text { Área tratada } \\
\qquad\left(\mathrm{cm}^{2}\right)\end{array}$} & 324 & 196 & 324 & 290,23 \\
\hline \multicolumn{2}{|c|}{$\begin{array}{l}\text { Área radiada } \\
\qquad\left(\mathrm{cm}^{2}\right)\end{array}$} & 529,59 & 447,24 & 589,67 & 545,77 \\
\hline \multicolumn{2}{|c|}{$\begin{array}{c}\text { Eficiencia } \\
(\%)\end{array}$} & 61,17 & 43,82 & 54,94 & 53,17 \\
\hline \multicolumn{2}{|c|}{$\begin{array}{c}\text { Centro de radiaciones } \\
\text { fuera de la zona a } \\
\text { tratar? }\end{array}$} & No & No & $\mathrm{Si}$ & $\mathrm{Si}$ \\
\hline \multicolumn{2}{|c|}{$\begin{array}{l}\text { No. radiaciones } \\
\text { acumuladas }\end{array}$} & 2 & 4 & 3 & 3 \\
\hline \multirow{4}{*}{$\begin{array}{c}\text { Área sin } \\
\text { cubrir } \\
\text { con } \\
\text { respecto } \\
\text { al modelo } \\
\left(\mathrm{cm}^{2}\right)\end{array}$} & $\mathbf{a}$ & - & - & 4,77 & 0,52 \\
\hline & b & - & - & - & - \\
\hline & c & 1,25 & - & - & - \\
\hline & d & 5,62 & - & - & - \\
\hline
\end{tabular}


El modelo (b) tiene menor eficiencia y zona tratada, adicionalmente tiene zonas con cuatro radiaciones acumuladas siendo peligroso para los limites de temperatura deseados en la madera. Por estas razones no se tiene en cuenta en la comparativa con los demás modelos.

Al comparar el modelo (a) y (c) que tienen zonas tratadas iguales y mayor eficiencia se ve que pierde menos zona tratada con solape el modelo (a). Adicionalmente el modelo (c) tiene zonas con tres radiaciones acumuladas y se pierde potencia de microondas al encontrarse 2 radiaciones fuera del área a tratar por cada $18 \mathrm{~cm}$ tratados, esto podría poner en peligro el entorno de la zona tratada.

Aunque en el modelo (d) se pierda menos zona tratada con solape en comparación con el modelo (a), en el modelo (d) además de los inconvenientes del modelo (c), se tiene una área tratada más pequeña con el mismo número de radiaciones y mayor tiempo.

En conclusión, el modelo (a) es más eficiente en cuanto a zona tratada, radiaciones, tiempo requerido y en consecuencia energéticamente; además da mayor seguridad para el entorno y la madera. Por lo tanto es éste modelo el que se utiliza para determinar la tasa de mortalidad, pero antes hay que hacer pruebas de temperatura sobre las zonas con dos radiaciones.

\subsubsection{Pruebas de temperatura para radiaciones solapadas}

El objetivo es garantizar que la temperatura de la madera siga por debajo de los límites fijados al principio de este capítulo. Con este objetivo se colocan sondas de temperatura en el interior de la madera en los puntos indicados en la Figura 3-15, donde:

1. Sonda 1: a $3,5 \mathrm{~cm}$ desde el centro en dirección perpendicular a las fibras.

2. Sonda 2: a $5 \mathrm{~cm}$ desde el centro en dirección perpendicular a las fibras.

3. Sonda 3: a $3,5 \mathrm{~cm}$ desde el centro en dirección paralela a las fibras.

4. Sonda 4: a $5 \mathrm{~cm}$ desde el centro en dirección paralela a las fibras.

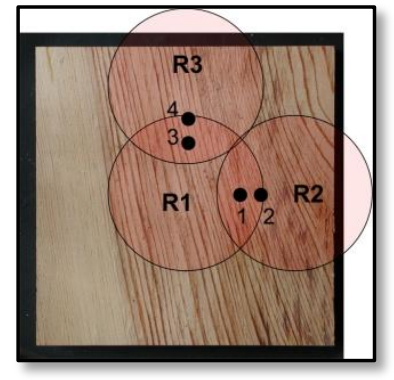

Figura 3-15. Localización de sondas en Solapes

El procedimiento es aplicar radiaciones de 20 segundos de duración, solapadas según el modelo (a) de la Figura 3-14 y espaciadas un tiempo conocido, radiándose primero en la dirección perpendicular a las fibras y luego en la dirección paralela; ya

60 Tecnología de microondas en el sector de restauración de bienes culturales 
que en ésta dirección se alcanza mayor temperatura en la aplicación inicial. El orden de aplicación se observa en la Figura 3-15, siendo R1, R2 y R3 las radiaciones consecutivas.

Se realiza un experimento con cinco muestras radiales y luego con cuatro muestras tangenciales. En el primero los tiempos de espera entre radiaciones varían de 20 a 35 segundos, por lo que de los datos adquiridos no se puede sacar una mediana con bajo índice de dispersión. En el segundo se espera 10 segundos para la primera radiación (R1), luego 25 segundos para la segunda radiación (R2) y finalmente 5 minutos para la última radiación (R3).

Tabla 3-9. Temperatura solapes en muestras radiales

\begin{tabular}{|c|c||}
\hline SONDA & $\begin{array}{c}\text { TEMPERATURA } \\
{ }^{\circ} \mathbf{C}\end{array}$ \\
\hline 1 & 36,5 \\
\hline 2 & 39,54 \\
\hline 3 & 49,48 \\
\hline 4 & 52,06 \\
\hline
\end{tabular}

Tabla 3-10. Temperatura solapes en muestras tangenciales

\begin{tabular}{||c|c||}
\hline SONDA & $\begin{array}{c}\text { TEMPERATURA } \\
{ }^{\mathbf{O}} \mathbf{C}\end{array}$ \\
\hline 1 & 39,78 \\
\hline 2 & 54,68 \\
\hline 3 & 47,20 \\
\hline 4 & 58,84 \\
\hline
\end{tabular}

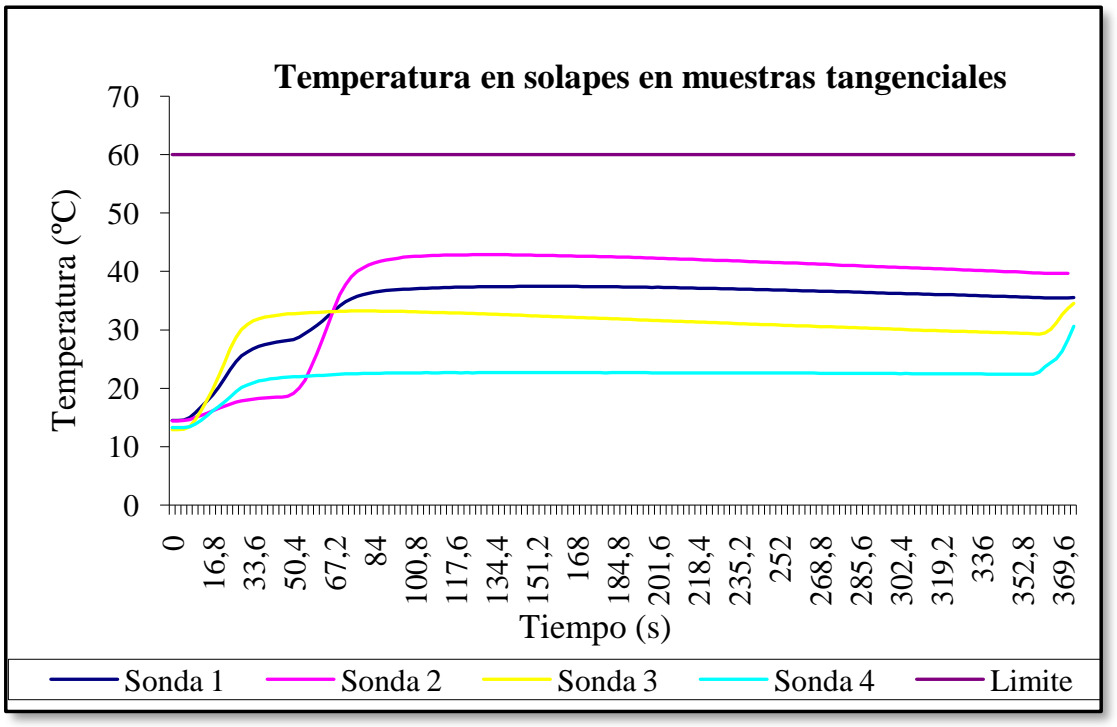

Figura 3-16.Temperatura solapes en muestras tangenciales

La Tabla 3-9 muestra la temperatura máxima alcanzada en los cuatro puntos controlados, demostrando que los niveles de temperatura en los solapes para muestras radiales no superan el límite de $60^{\circ} \mathrm{C}$ propuesto en un principio. También se observa una diferencia de temperatura de aproximadamente $10^{\circ} \mathrm{C}$ entre los puntos superiores (3 y 4) y los laterales (1 y 2). 
En cuanto a las tablas tangenciales, en la Tabla 3-10 se lista la temperatura máxima alcanzada en cada punto y en la Figura 3-16 se muestra la mediana de la temperatura en cada punto para las muestras utilizadas; la temperatura alcanzada está por debajo del límite de $60^{\circ} \mathrm{C}$. De nuevo la temperatura en los puntos superiores (3 y 4) es mayor que la de los puntos laterales ( 1 y 2), sin importar que el tiempo de espera para la radiación (R3) se incrementara considerablemente.

En la Figura 3-17 se muestra el índice de dispersión para los datos de la Figura 3-16. La mayor dispersión se encuentra cerca de los puntos de encendido y apagado de la antena, causado por la falta de precisión en el procedimiento manual de encendido del aplicador y la toma de tiempo.

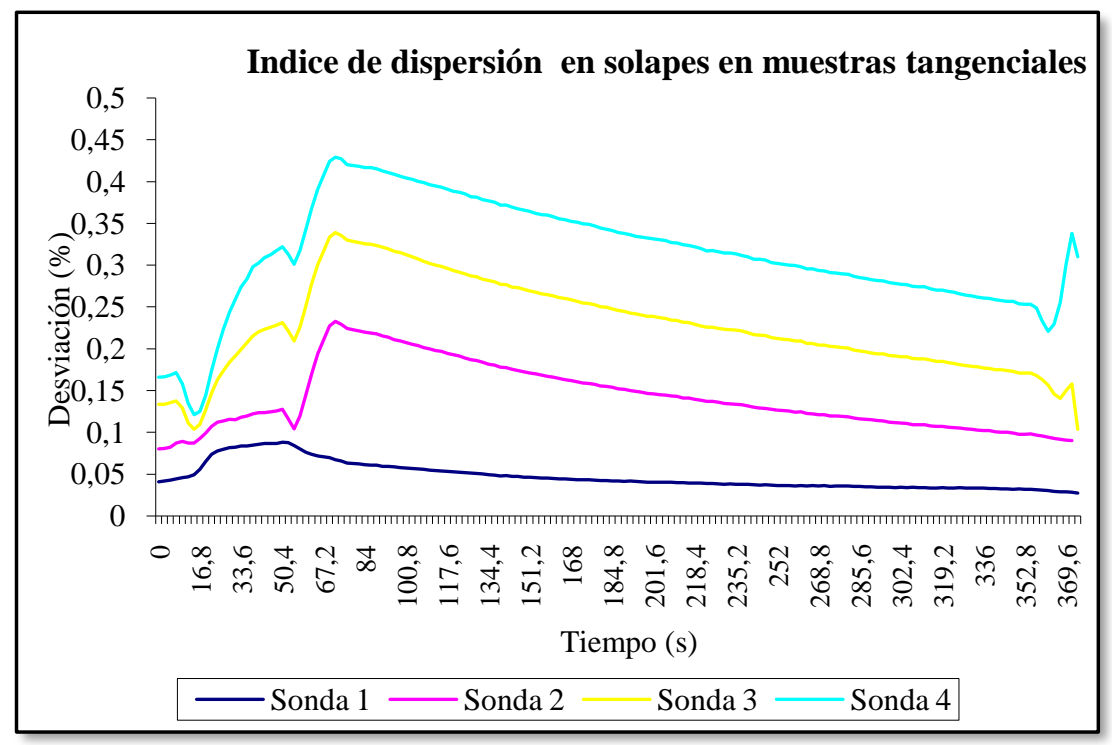

Figura 3-17. Índice de dispersión solapes muestras tangenciales

\subsubsection{Tasa de Mortalidad con radiaciones solapadas}

Una vez comprobado que la madera no está expuesta a temperaturas peligrosas en los solapes del procedimiento propuesto, el siguiente paso es hacer tres pruebas para observar la tasa de mortalidad:

1. Prueba 1: consta de tres radiaciones de 20 segundos cada una y con espacios de tiempo entre radiaciones de 25 segundos en la dirección perpendicular a las fibras y 5 minutos en dirección paralela a las fibras. Se colocan seis larvas en cada muestra según se indica en la Figura 3-18, utilizando dos muestras radiales y dos tangenciales, la población de control está compuesta por 13 larvas y el peso de las larvas varía entre 50 y 250 mg. El total de larvas tratadas es 24.

2. Prueba 2: consta de cuatro radiaciones de 20 segundos con espacios de tiempo entre radiaciones de 20 segundos en la dirección perpendicular a las fibras y 5 minutos en la dirección paralela a las fibras. Se colocan 6 larvas 
en cada muestra como se muestra en la Figura 3-19, utilizando nuevamente dos tablas tangenciales y dos radiales, la población de control es de 11 larvas y el peso de las larvas varía entre 50 y $100 \mathrm{mg}$. Se tratan 24 larvas en total.

3. Prueba 3: cuatro radiaciones igual a la prueba 2, pero el peso de las larvas varía entre 100 y $200 \mathrm{mg}$, con una población de control de 10 larvas y un total de 24 larvas tratadas.

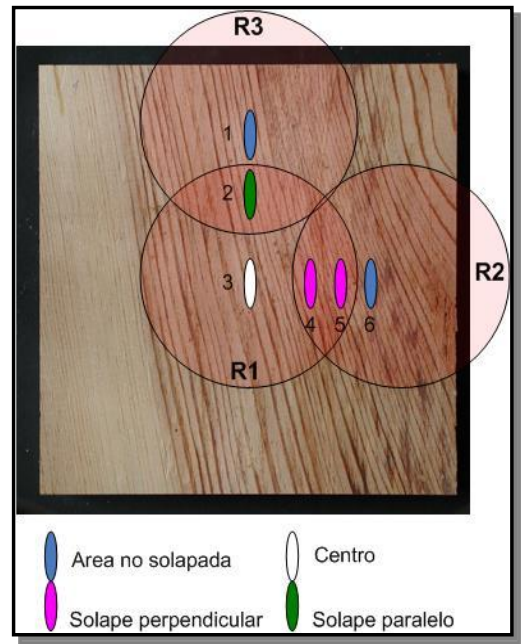

Figura 3-18. Localización larvas en tres radiaciones

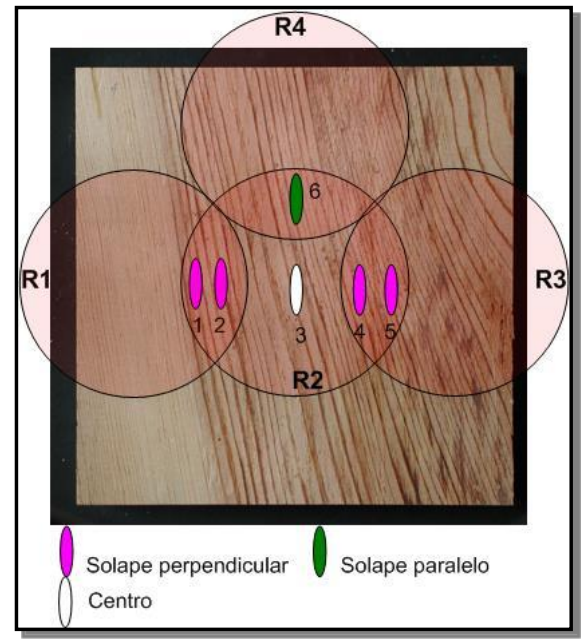

Figura 3-19. Localización larvas en cuatro radiaciones

Los resultados de la tasa de mortalidad de estas tres pruebas en relación con las larvas tratadas se muestran en la Tabla 3-11. Puede verse que el tratamiento es $100 \%$ efectivo en todos los puntos excepto en el punto solape perpendicular, hecho que puede explicarse por el factor de forma del campo eléctrico del aplicador; adicionalmente, las larvas localizadas en el solape perpendicular izquierdo (1 y 2) tienen menor tasa de mortalidad que las localizadas en el lado derecho.

En el Apéndice II.ii se detalla la observación de cada larva tratada y de la población de control, que en los tres casos supera el $93 \%$ de supervivencia, validando de esta manera los resultados.

En resumen, la efectividad del tratamiento con este procedimiento aumenta a $72,22 \%$ de mortalidad inmediata, 20\% más que con una aplicación central después de 70 días de observación. Con el objetivo de lograr una mayor efectividad del procedimiento se plantea la construcción de un aplicador con polarización circular (tema del siguiente capítulo). De esa manera, la orientación del campo eléctrico es independiente de la orientación de las fibras y se espera contrarrestar el bajo índice de mortalidad en el solape perpendicular. 
Tabla 3-11. Tasa de mortalidad con radiación solapada

\begin{tabular}{|c|c|c|c|c|c|c|}
\hline \multirow{3}{*}{ LOCALIZACIÓN } & \multirow{2}{*}{\multicolumn{2}{|c|}{$\begin{array}{c}3 \text { Radiaciones } \\
50-250 \mathrm{mg}\end{array}$}} & \multicolumn{4}{|c|}{4 Radiaciones } \\
\hline & & & \multicolumn{2}{|c|}{$50-100 \mathrm{mg}$} & \multicolumn{2}{|c|}{$100-200 \mathrm{mg}$} \\
\hline & $\begin{array}{l}\text { No. } \\
\text { Larvas }\end{array}$ & T.M. & $\begin{array}{l}\text { No. } \\
\text { Larvas }\end{array}$ & T.M. & $\begin{array}{l}\text { No. } \\
\text { Larvas }\end{array}$ & T.M. \\
\hline Área no solapada & 8 & $\begin{array}{c}100 \% \\
8 / 8\end{array}$ & - & - & - & - \\
\hline Centro & 4 & $\begin{array}{c}100 \% \\
4 / 4\end{array}$ & 4 & $\begin{array}{c}100 \% \\
4 / 4\end{array}$ & 4 & $\begin{array}{c}100 \% \\
4 / 4\end{array}$ \\
\hline $\begin{array}{c}\text { Solape } \\
\text { Perpendicular }\end{array}$ & 8 & $\begin{array}{c}37,5 \% \\
3 / 8\end{array}$ & 16 & $\begin{array}{c}43,75 \% \\
7 / 16\end{array}$ & 16 & $\begin{array}{c}62,5 \% \\
10 / 16\end{array}$ \\
\hline Solape Paralelo & 4 & $\begin{array}{c}100 \% \\
4 / 4\end{array}$ & 4 & $\begin{array}{c}100 \% \\
4 / 4\end{array}$ & 4 & $\begin{array}{c}100 \% \\
4 / 4\end{array}$ \\
\hline
\end{tabular}

Para finalizar con lo propuesto en este capítulo, queda por comprobar que ni la estructura ni la imprimación sean afectadas por un tratamiento de microondas durante 20 segundos.

\subsection{EFECTO DEL TRATAMIENTO}

En este apartado se usan muestras tangenciales radiadas con la dirección del campo eléctrico orientado paralelo a la dirección de las fibras, teniendo en cuenta que según el apartado 3.2 es el caso donde se alcanzan mayores temperaturas. Se estudia el efecto de un tratamiento de 20 segundos con $900 \mathrm{~W}$ de potencia sobre la estructura, la imprimación, listones y nudos.

\subsubsection{Efecto sobre la estructura}

La temperatura máxima alcanzada para cada sonda de la Figura 3-6 se muestra en la Tabla 3-12. La temperatura máxima se alcanza en el centro de la radiación y ésta se encuentra por debajo del límite propuesto para esta investigación. La temperatura en los tres puntos restantes es similar debido a su cercanía con el margen de la muestra, aunque la de la diagonal sea ligeramente inferior.

La temperatura en los puntos periféricos permite concluir que en el peor de los casos (las esquinas), el punto perpendicular a las fibras tendrá la misma temperatura que el punto paralelo a ellas. En el Apéndice II.iii se detallan las medidas de temperatura hechas. 
Tabla 3-12. Temperatura pruebas de estructura

\begin{tabular}{|c|c||}
\hline SONDA & $\begin{array}{c}\text { TEMPERATURA } \\
\left({ }^{\circ} \mathbf{C}\right)\end{array}$ \\
\hline 1 & 28,36 \\
\hline 2 & 27,02 \\
\hline 3 & 53,56 \\
\hline 4 & 28,80 \\
\hline
\end{tabular}

La variación media obtenida con el comparador centesimal en cada uno de los 9 puntos se muestra en la Tabla 3-13, debido a la posibilidad que la muestra no esté perfectamente colocada sobre una superficie lisa, la incertidumbre de las medidas es $\pm 0,1 \mathrm{~mm}$.

Los movimientos no son significativos, ya que los valores obtenidos son comparables con la incertidumbre de la medida. Por lo tanto no constituyen riesgo alguno para la buena conservación de la madera o la pintura sobre ella.

Tabla 3-13. Variaciones pruebas de estructura

\begin{tabular}{|c|c|c|c|c|c|c|c|c|c|}
\hline & A1 & A2 & A3 & B1 & B2 & B3 & C1 & C2 & C3 \\
\hline $\begin{array}{c}\Delta \\
(\mathrm{mm})\end{array}$ & 0,124 & 0,122 & 0,188 & 0,128 & 0,102 & 0,202 & 0,122 & 0,098 & 0,173 \\
\hline
\end{tabular}

\subsubsection{Efecto sobre la imprimación}

Tabla 3-14. Temperaturas máximas pruebas imprimación

\begin{tabular}{||c|c|c|c|c|c|c||}
\hline \multirow{2}{*}{ Tipo Tabla } & \multicolumn{3}{|c|}{ R1 } & \multicolumn{3}{c||}{ R2 } \\
\cline { 2 - 7 } & $\begin{array}{c}\text { Interna } \\
{ }^{\mathbf{0}} \mathbf{C}\end{array}$ & $\begin{array}{c}\text { Madera } \\
{ }^{\mathbf{}} \mathbf{C}\end{array}$ & $\begin{array}{c}\text { Imprim. } \\
{ }^{\mathbf{o}} \mathbf{C}\end{array}$ & $\begin{array}{c}\text { Interna } \\
{ }^{\mathbf{}} \mathbf{C}\end{array}$ & $\begin{array}{c}\text { Imprim. } \\
{ }^{\mathbf{}} \mathbf{C}\end{array}$ & $\begin{array}{c}\text { Madera } \\
{ }^{\mathbf{}} \mathbf{C}\end{array}$ \\
\hline $\begin{array}{c}\text { Sin } \\
\text { Imprimación }\end{array}$ & 55,49 & 40,78 & 36,51 & - & - & - \\
\hline Imprimación & 53,7 & 35,84 & 36,56 & 54,46 & 33,04 & 36,56 \\
\hline $\begin{array}{c}\text { Imprimación } \\
\text { con tela }\end{array}$ & 50,73 & 36,35 & 27,96 & 54,01 & 26,27 & 32,22 \\
\hline $\begin{array}{c}\text { Imprimación } \\
\text { con estopa }\end{array}$ & 49,88 & 38,26 & 25,18 & 55,36 & 27,06 & 42,09 \\
\hline \hline
\end{tabular}


Las temperaturas máximas alcanzadas después de un tratamiento de 20 segundos sobre muestras con diferentes tipos de imprimación se muestran en la Tabla 3-14, donde se clasifican según tipo de muestra y la cara de radiación. R1 indica que se radia por la cara sin imprimación (madera) y $\mathrm{R} 2$ que se radia sobre la cara con imprimación. Los datos completos de estas medidas se muestran en el Apéndice II.iii.

La temperatura alcanzada en la imprimación es similar para los dos casos de radiación en cada tipo de muestra y los valores están por debajo del límite de $55{ }^{\circ} \mathrm{C}$ planteado para la superficie pintada, permitiendo concluir que el efecto del tratamiento sobre la imprimación no representa ningún riesgo.

Las siguientes tres figuras representan la temperatura alcanzada en cada punto de control para los diferentes tipos de muestra, diferenciando la cara por la que se ha aplicado el tratamiento.

En la Figura 3-20 se presentan los resultados del punto central sobre la cara de radiación, y en ella se puede apreciar que la temperatura alcanzada en la cara de radiación cuando se radia directamente sobre la madera es mayor que la que se alcanza cuando se hace sobre la imprimación. Adicionalmente, cuando las muestras con imprimación cuentan con un sustrato de tela o estopa la temperatura sobre la imprimación disminuye notablemente en comparación con las muestras que no lo tienen. Lo que se puede concluir es que el sustrato no se calienta por el efecto de las microondas, como era de esperar y como resultado la temperatura promedio alcanzada en la superficie es menor en su presencia. Por otro lado, la temperatura en la cara de radiación en muestras sin imprimación es ligeramente mayor que en las que la tienen.

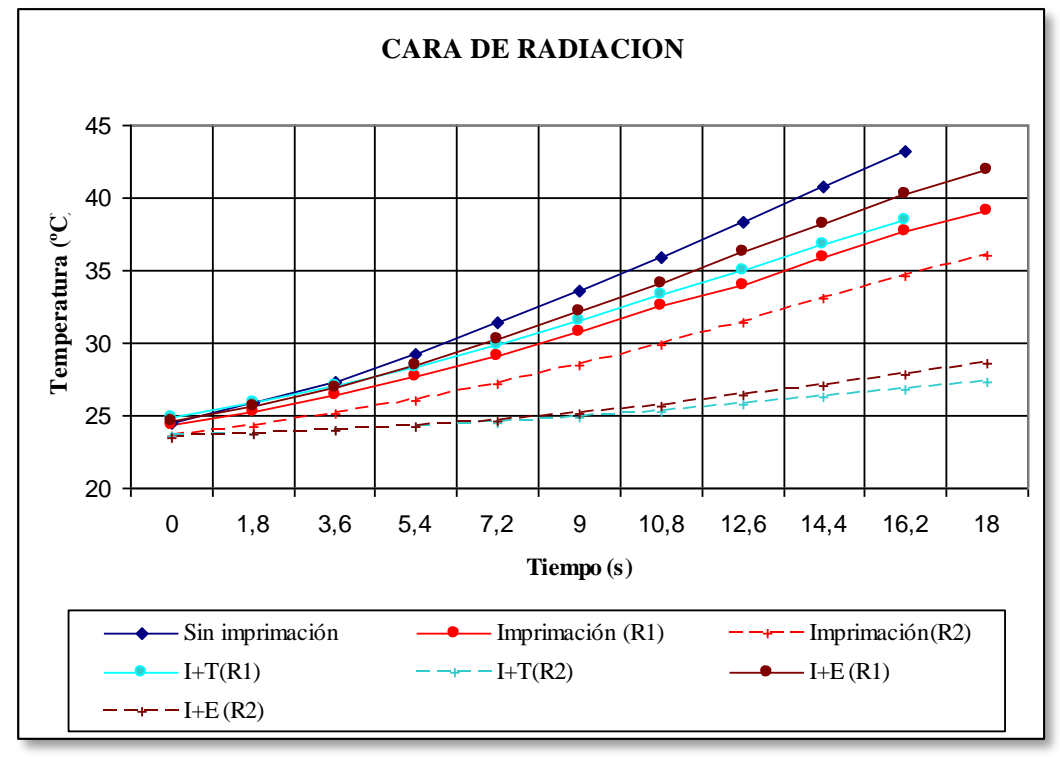

Figura 3-20. Temperatura en la cara de radiación 
En la Figura 3-21 se presentan los resultados del punto central sobre la cara posterior a la de radiación, y en ella se observa que la temperatura alcanzada en la cara posterior cuando se radia directamente sobre la imprimación con sustrato es mayor que la que se alcanza cuando se hace sobre la madera; es decir, la temperatura en la imprimación con sustrato es menor que en la madera como sucedía en el caso anterior. Por otro lado, no es apreciable una diferencia de temperatura entre las muestras con y sin imprimación.

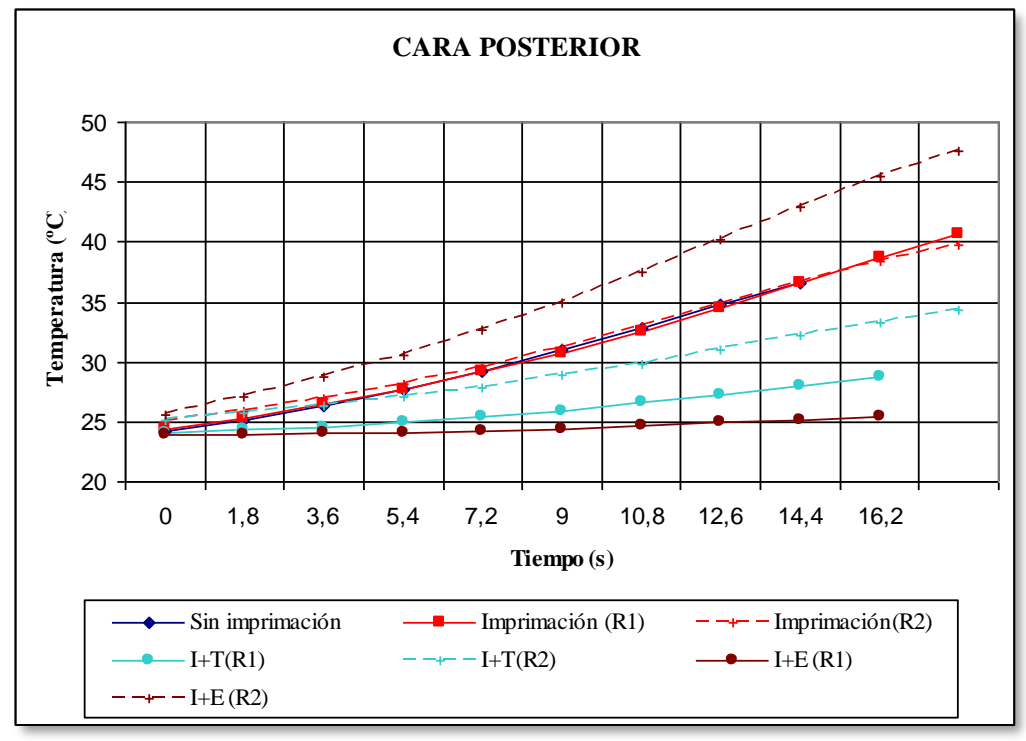

Figura 3-21. Temperatura en la cara posterior

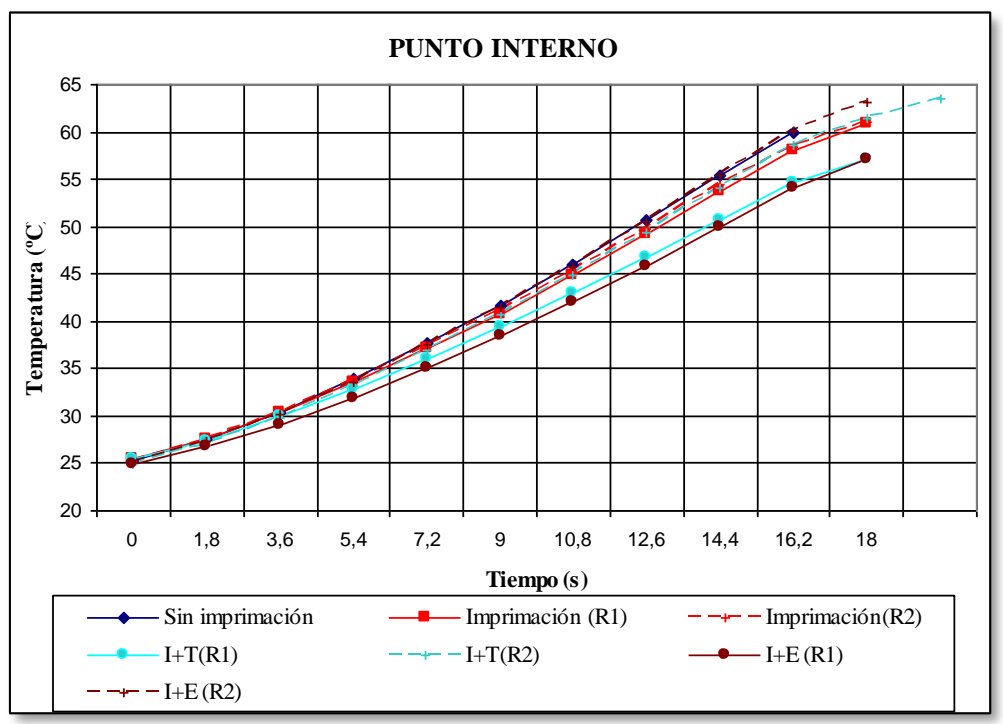

Figura 3-22.Temperatura en el punto interior 
En la Figura 3-22 se presentan los resultados del punto central interior. En este caso no se observa una gran diferencia de temperatura entre los diferentes tipos de muestras.

En conclusión, la temperatura está por debajo del límite de $55^{\circ} \mathrm{C}$ en la imprimación, sin importar la cara por la que se aplique el tratamiento de microondas. Cuando las muestras tienen sustrato, como tela o estopa, la temperatura en la imprimación está por debajo de $\operatorname{los} 30^{\circ} \mathrm{C}$. Adicionalmente la temperatura en la cara de la imprimación siempre es menor que en la cara de la madera independientemente de dónde se aplique el tratamiento, lo cual permite inferir que el tratamiento de 20 segundos no pone en peligro la superficie de la pintura.

A pesar de los resultados anteriores no se puede afirmar que la tela o la estopa dentro de la imprimación tengan propiedades aislantes. En la Tabla 3-15 se muestra un cuadro comparativo de los resultados a 20 segundos (obtenidos en el apartado 3.2) y el valor de temperatura en cada punto para la totalidad de las muestras tratadas en estas pruebas, diferenciando entre las que fueron tratadas por la cara de la madera (R1) y las que lo fueron por la imprimación (R2).

Tabla 3-15. Comparación de temperaturas pruebas imprimación

\begin{tabular}{|c|c|c|c|c|c|c||}
\hline \multirow{2}{*}{} & \multicolumn{2}{|c|}{$\begin{array}{c}\text { PUNTO } \\
\text { INTERIOR }\end{array}$} & \multicolumn{2}{c|}{$\begin{array}{c}\text { CARA } \\
\text { RADIACIÓN }\end{array}$} & \multicolumn{2}{c|}{$\begin{array}{c}\text { CARA } \\
\text { POSTERIOR }\end{array}$} \\
\cline { 2 - 7 } & $\mathbf{T}^{\mathbf{}} \mathbf{C}$ & $\mathbf{I d}^{\mathbf{4}}$ & $\mathbf{T}^{\mathbf{}} \mathbf{C}$ & Id $^{\prime}$ & $\mathbf{T}^{\mathbf{}} \mathbf{C}$ & Id \\
\hline Fase I & 42 & 0,113 & 31,06 & 0,134 & 31,48 & 0,144 \\
\hline $\mathbf{R 1}$ & 52,22 & 0,043 & 37,31 & 0,053 & 32,24 & 0,158 \\
\hline $\mathbf{R 2}$ & 54,46 & 0,010 & 27,06 & 0,128 & 36,56 & 0,111 \\
\hline
\end{tabular}

El índice de dispersión encontrado en estas medidas es inferior al encontrado en las pruebas hechas en la primera parte de esta investigación, por lo que puede decirse que la imprimación no repercute en el tratamiento o en los resultados encontrados anteriormente. También se puede afirmar a la luz de los resultados encontrados que aplicar el tratamiento sobre la imprimación o su cara opuesta no implica diferencia alguna en los resultados esperados ni en los efectos que las microondas puedan causar sobre la imprimación.

Lo anterior también es confirmado por los resultados de las termografías, mostrados en la Figura 3-23, donde se puede observar que el perfil de temperaturas internas es muy similar sin importar la cara en la que se aplique el tratamiento de microondas (R1 ó R2) y sin importar el tipo de imprimación o substrato que se haya aplicado sobre la madera (imprimación, imprimación y tela, e imprimación y estopa). Las muestras se comportan de forma muy similar por lo que puede inferirse que sin importar el tipo de substrato o imprimación, el perfil de temperaturas dentro de

${ }^{4}$ Índice de dispersión.

68 Tecnología de microondas en el sector de restauración de bienes culturales 
madera será el mismo y por lo tanto los efectos que tendrá el tratamiento serán similares.

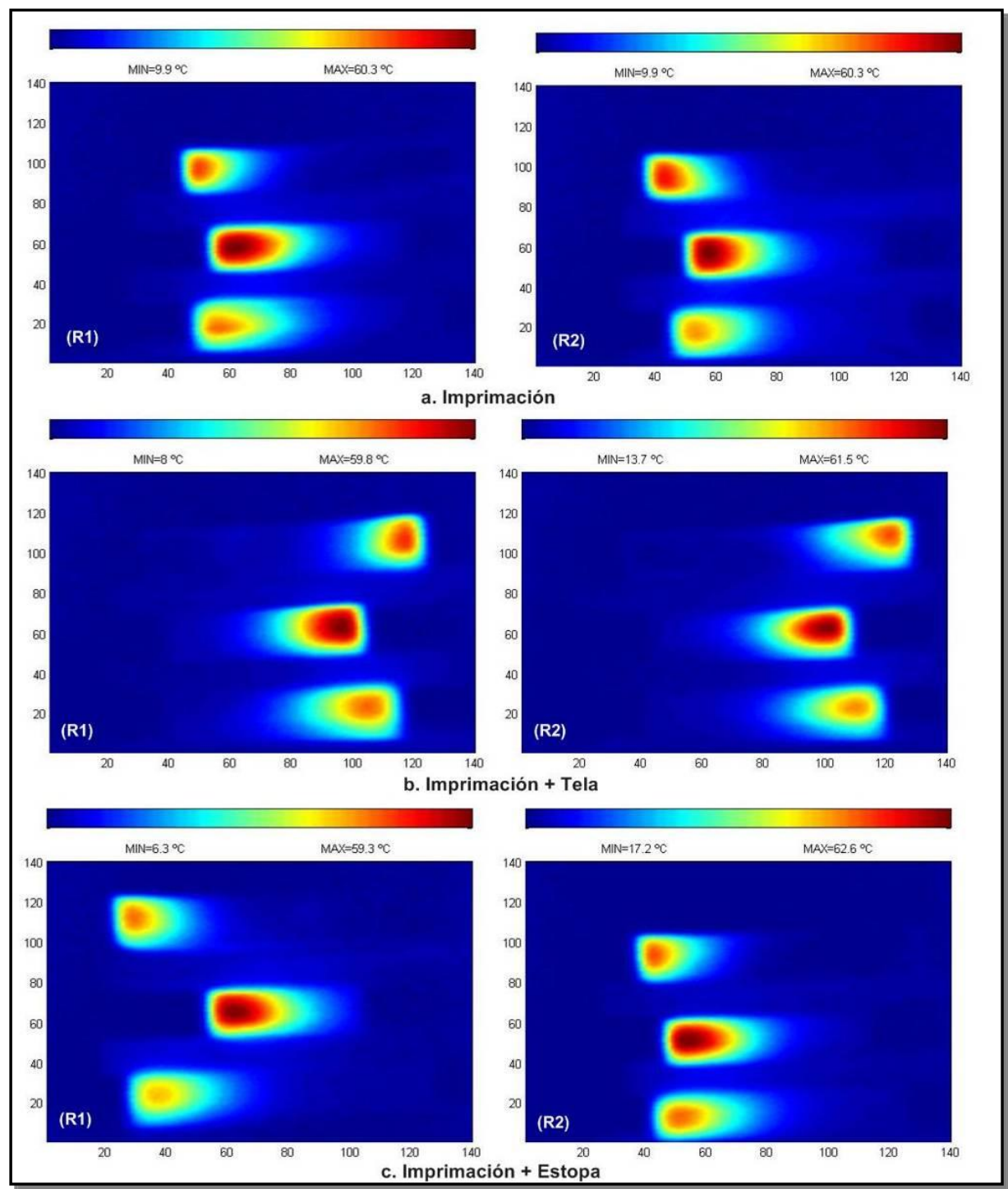

Figura 3-23. Termografías internas muestras con imprimación

Finalmente en la Figura 3-24 se muestra la termografía hecha a las muestras sin imprimación, aunque se espere que el perfil de calentamiento en la cara de radiación y en la cara opuesta tenga forma circular o elíptica, éste depende de las fibras de cada madera por lo que estas formas se ven un poco deformadas cuando se habla de temperatura y no de campo eléctrico. En el perfil de las caras internas se puede distinguir que la cara paralela a las fibras (cara 1) tiene una mayor profundidad que las perpendiculares (caras 2 y 3 ) mostrando el efecto elíptico esperado por la 
orientación del campo eléctrico. Esto no se ve en las demás termografías internas y puede deberse a la falta de precisión al hacer coincidir el centro del corte de la muestra y el centro de la antena, dando como resultado un mayor calentamiento en la cara 2 que en las dos restantes.

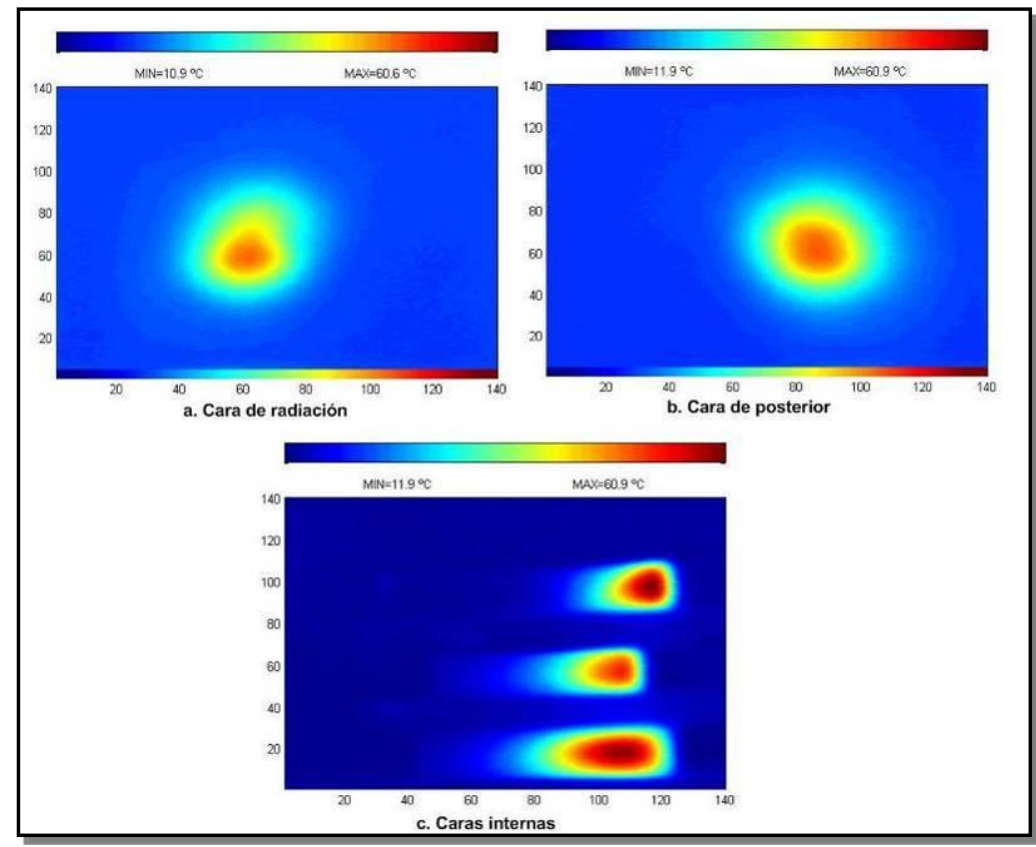

Figura 3-24.Termografías muestras sin imprimación

Tabla 3-16. Relación de Termografías

\begin{tabular}{||c|c|c|c|c|}
\hline Muestra & Termografía & Tipo de Tabla & $\begin{array}{c}\text { T Máx. } \\
{ }^{\mathbf{}} \mathbf{C}\end{array}$ & $\begin{array}{c}\text { T Mín. } \\
{ }^{\mathbf{} C}\end{array}$ \\
\hline T28 & Cara radiación & Sin imprimación & 34,7 & 22,3 \\
\hline T29 & Cara radiación & Sin imprimación & 63,7 & 20,2 \\
\hline T29 & Cara posterior & Sin imprimación & 65,4 & 24,6 \\
\hline T1 & Cara aplicación & Sin imprimación & 60,6 & 10,9 \\
\hline T1 & Cara posterior & Sin imprimación & 60,9 & 11,9 \\
\hline T2 & Caras internas & Sin imprimación & 61,9 & 15,1 \\
\hline T3 & Caras internas & Sin imprimación & 60 & 8,8 \\
\hline T4 & Caras internas & Sin imprimación & 63,7 & 20,4 \\
\hline T11 & Caras internas & Con imprimación & 60,3 & 9,9 \\
\hline T12* & Superficie aplicación & Con imprimación & 59 & 4,9 \\
\hline T12* & Superficie posterior & Con imprimación & 59,3 & 6,3 \\
\hline T12* & Caras internas & Con imprimación & 60,3 & 9,9 \\
\hline T21 & Superficie aplicación & Con I+Tela & 43,9 & 22 \\
\hline T21 & Superficie aplicación & Con I+Tela & 59,6 & 7,2 \\
\hline T21 & Caras internas & Con I+Tela & 59,8 & 8 \\
\hline T22 & Caras internas & Con I+Tela & 57,5 & $-1,4$ \\
\hline T23* & Superficie aplicación & Con I+Tela & 43,4 & 21,1 \\
\hline T23* & Superficie posterior & Con I+Tela & 59,8 & 8 \\
\hline \hline
\end{tabular}




\begin{tabular}{||c|c|c|c|c||}
\hline \hline T23* & Caras internas & Con I+Tela & 61,5 & 13,7 \\
\hline T24* & Caras internas & Con I+Tela & 59,8 & 8 \\
\hline T31 & Superficie aplicación & Con I+Estopa & 44,7 & 23,2 \\
\hline T31 & Superficie posterior & Con I+Estopa & 59 & 4,9 \\
\hline T31 & Caras internas & Con I+Estopa & 59,3 & 6,3 \\
\hline T32* & Superficie aplicación & Con I+Estopa & 58,4 & 2,4 \\
\hline T32* & Superficie posterior & Con I+Estopa & 59,6 & 7,2 \\
\hline T32* & Caras internas & Con I+Estopa & 62,6 & 17,2 \\
\hline \hline
\end{tabular}

* Muestras tratadas por la cara de la imprimación (R2).

Sólo se ha mostrado las termografías que se consideraran más representativas, mientras que la relación de muestras se publica en la Tabla 3-16, donde adicionalmente se indican las temperaturas máxima y mínima alcanzadas.

\subsection{TEMPERATURA ADICIONAL}

En este apartado el objetivo es observar la temperatura alcanzada en listones y nudos, bajo un tratamiento de microondas con las mismas características mencionadas durante el desarrollo de este capítulo y con una duración de 50 segundos en el caso de los listones y 25 segundos en el caso de los nudos. Para la toma de datos se esperan 5 segundos para encender el prototipo de desinsectación.

\subsubsection{Temperatura en listones}

En la Figura 3-25 se muestran los resultados de temperatura en el interior de los listones. Se observa que la temperatura aumenta más rápidamente en el punto más cercano a la radiación y que una vez se alcanzan los $100^{\circ} \mathrm{C}$ aumentar la temperatura es difícil; la diferencia con los resultados en tablas se debe a que el calentamiento mediante microondas está relacionado con el volumen del objeto a calentar.

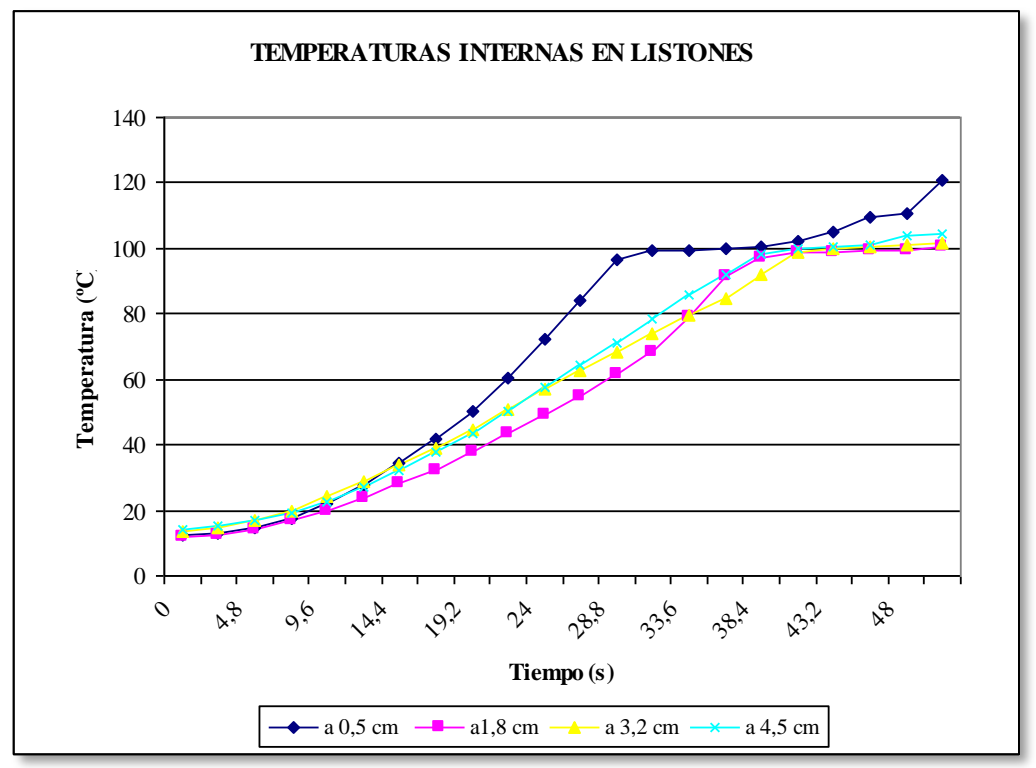

Figura 3-25. Temperatura interna en listones 
Por otro lado, el índice de dispersión alcanza el $27 \%$ en el punto más cercano a la radiación, mientras en los demás no supera el 20\%; pero estos índices son alcanzados durante el encendido del prototipo, por lo que pueden deberse a la falta de exactitud al repetir las medidas con diferentes muestras.

En conclusión, con un tratamiento de 20 segundos la temperatura en el interior de los listones está por debajo del límite de $60^{\circ} \mathrm{C}$, siendo este un tratamiento seguro también para los elementos constitutivos de los retablos.

La Figura 3-26 muestra los resultados de temperatura en las cuatro superficies de los listones. La temperatura en los lados superior y posterior es mayor que en los laterales, donde las temperaturas son muy parecidas.

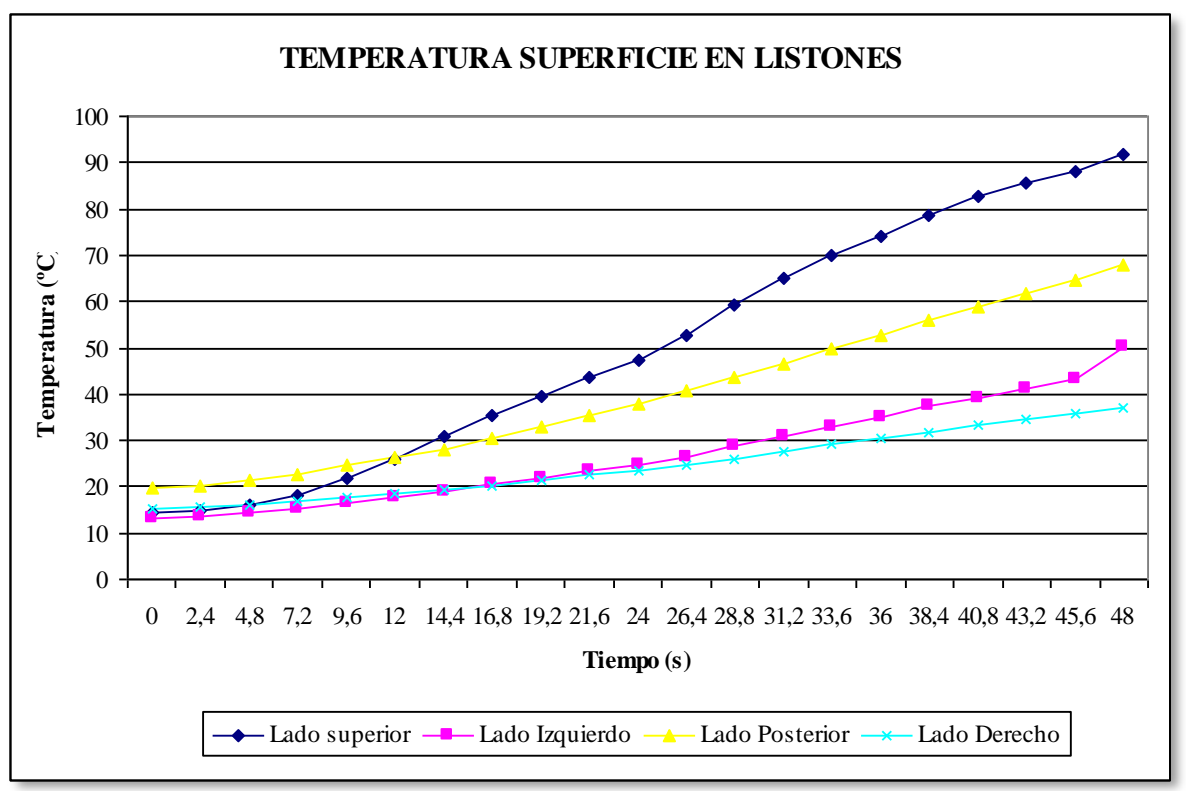

Figura 3-26. Temperatura Superficial en listones

En cuanto a la validez de estos resultados, el índice de dispersión se encuentra por debajo del $20 \%$, excepto para el lado izquierdo que aumenta hasta un máximo del $30 \%$. Esto se debe a que sólo en esta cara la temperatura es mucho mayor para el listón 7 que la de los demás listones observados. Los resultados de las medidas se muestran en el Apéndice II.iv.

Por último, destacar que después de 20 segundos de tratamiento la temperatura en las superficies es inferior al límite de $55^{\circ} \mathrm{C}$ planteado, por lo que se concluye que el tratamiento sigue siendo seguro en la superficie de estos elementos constitutivos.

\subsubsection{Temperatura sobre nudos}

Para finalizar con este apartado, se mide la temperatura alcanzada directamente sobre los nudos durante un tratamiento de 25 segundos. 
Los resultados se muestran en la Figura 3-27, donde puede verse que la temperatura es inferior a $30^{\circ} \mathrm{C}$, tanto para el nudo en la parte posterior como para la superficie en la cara de radiación, lo que indica que si la madera presenta nudos el tratamiento sigue siendo seguro en estas zonas.

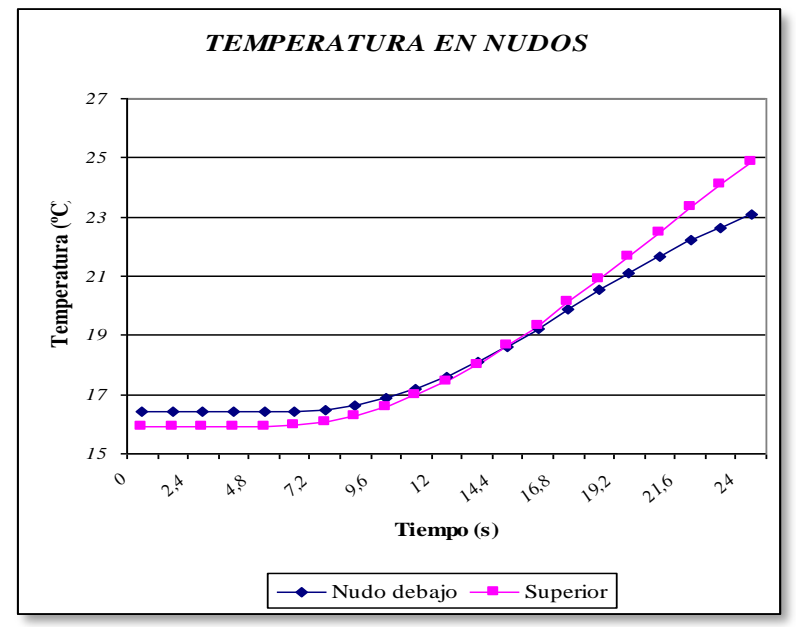

Figura 3-27. Temperatura sobre nudos

Sin embargo cada nudo se comporta de diferente modo y estos resultados no pueden considerarse estadísticamente representativos, debido a que sólo se han tratado dos muestras y a la disparidad de los resultados. Ahora bien, sí puede considerarse un dato orientativo para afirmar que la temperatura en los nudos es menor que en la madera, lo cual puede explicarse por la falta de moléculas de agua en los mismos.

\subsection{CONCLUSIÓN}

Se ha encontrado un procedimiento inicial para la desinsectación de retablos pintados mediante la utilización de energía de microondas, con una efectividad del $72 \%$. Este procedimiento consta de un tratamiento solapado de 20 segundos y una potencia de microondas de $900 \mathrm{~W}$.

No poder alcanzar un tratamiento $100 \%$ efectivo se debe a la baja efectividad del tratamiento en el área de solapamiento perpendicular, circunstancia que se entiende porque el diámetro mayor de la elipse que forma el campo eléctrico está orientado con la dirección de las fibras, esto hace que un solapamiento de igual magnitud en las áreas paralela y perpendicular sea insuficiente para lograr los mismo resultados. Para solucionar este problema y con el fin de lograr mayor homogeneidad e independencia de la orientación de las fibras, se plantea la construcción de un nuevo prototipo con polarización circular.

También se ha comprobado que este procedimiento es seguro para la madera (tanto en tablas como en listones) y la imprimación. Adicionalmente, se puede inferir que las zonas con nudos no representan ningún riesgo para el tratamiento de la madera. 
La experimentación fue hecha con un aplicador lineal y es posible que la utilización de otro tipo de aplicador u otra potencia lleve a diferentes resultados; de igual manera si se utiliza carcoma en otros estados de desarrollo la tasa de mortalidad puede variar. Esto no afecta al hecho que las microondas sean un medio viable y seguro para la desinsectación de retablos. 


\section{CAPÍTULO 4. DESARROLLO DE UN APLICADOR PARA DESINSECTACION DE MADERA}

\subsection{METODOLOGÍA}

Como continuación del capítulo anterior y con el fin de conseguir una mayor tasa de mortalidad dentro de las maderas, se plantea la necesidad de desarrollar un sistema de desinsectación por microondas con polarización circular; este sistema debe ser altamente versátil para poder tratar tanto obras de arte muy delicadas y de tamaño pequeño o mediano, como grandes estructuras de madera (vigas, artesonados, retablos, etc.). Este proyecto se idea a partir del interés de la empresa Desinsección y secado en 3D S.L. en utilizar la tecnología de microondas en el tratado de madera.

El sistema de desinsectación por microondas (Figura 4-1) consta de:

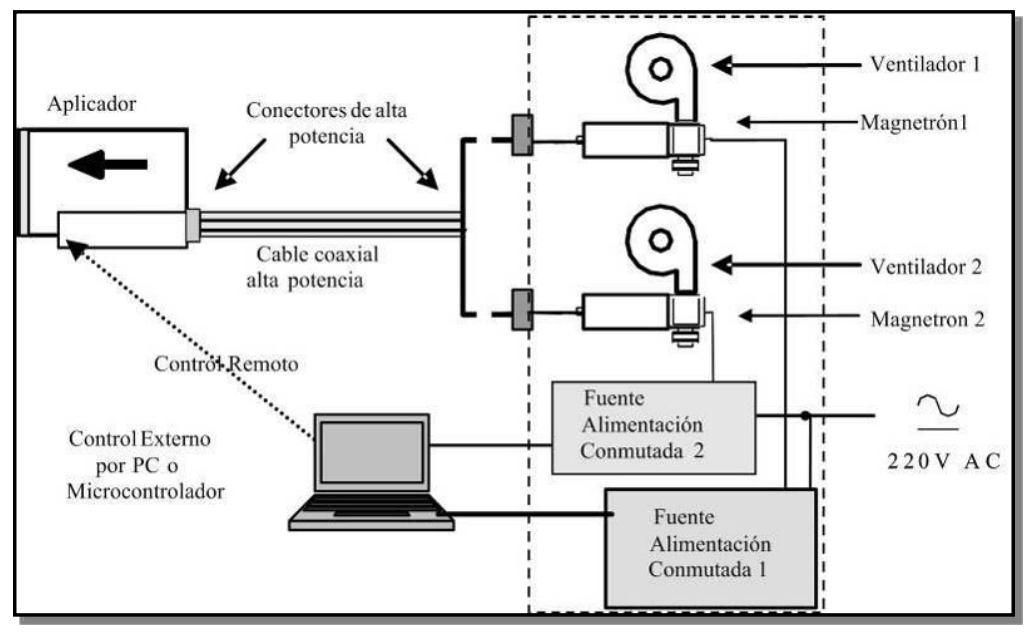

Figura 4-1. Sistema de desinsectación por microondas

- Un generador compuesto por:

- Dos fuentes conmutadas controladas por un micro controlador.

- Dos magnetrones controlados individualmente, que cuentan con ciclos de potencia entre $50 \mathrm{~W}$ y $1,3 \mathrm{~kW}$

- Un aplicador con dos tipos de funcionamiento:

- Modo A: la potencia podrá alcanzar los $2 \mathrm{~kW}$, para estructuras grandes. 
- Modo B: la potencia radiada será menor a $1 \mathrm{~kW}$, para obras delicadas.

El aplicador se conecta al generador mediante un cable coaxial de alta potencia que transporta la potencia necesaria; además cuenta con un brazo articulado cuya función es dar seguridad al operario durante el tratamiento de piezas y accesibilidad a las esquinas, alturas y puntos ocultos.

Teniendo en mente que el sistema debe permitir el tratamiento in situ de estructuras arquitectónicas y obras de arte, que por su tamaño y/o ubicación no se pueden mover para ser tratadas, las características que debe presentar son:

- Seguro: cumpliendo la normativa vigente sobre el límite de la exposición de las personas a campos electromagnéticos y evitando que el operario lo opere manualmente [36].

- Compacto: para facilitar su transporte, las maniobras del operario y su utilización en cualquier ambiente y para cualquier tamaño de elemento. También proporcionando una alta fiabilidad.

- Aplicador manejable: tamaño reducido que permita cierta facilidad en el montaje y desmontaje sobre el brazo articulado.

- Alta automatización: facilita el funcionamiento del sistema, homogeniza el método de aplicación, reduce errores y costos de funcionamiento.

- Uniformidad de campo: con la polarización circular se asegura una intensidad de campo más homogénea en la zona a tratar, permitiendo reducir el tiempo de tratamiento.

El proyecto se plantea en tres grandes etapas (Figura 4-2): Diseño, Construcción del Prototipo y Demostración; cada una de ellas está descompuesta en fases y es llevada a cabo por grupos de trabajo diferentes.

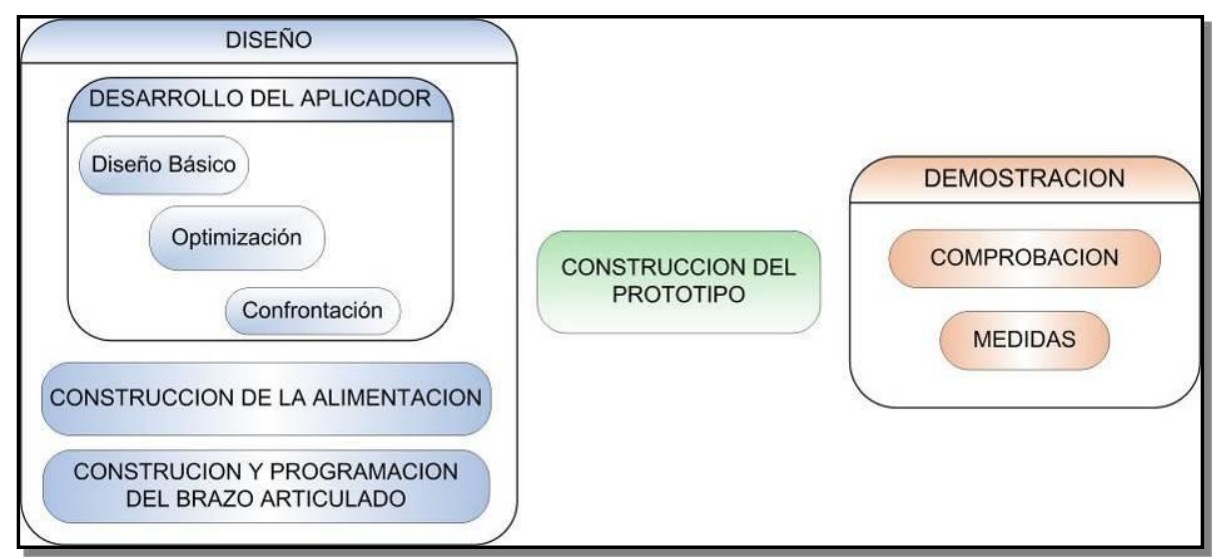

Figura 4-2. Etapas del proyecto

76 Tecnología de microondas en el sector de restauración de bienes culturales 
En este capítulo se tratan el desarrollo del aplicador y la etapa de demostración. Para la primera se tienen en cuenta las condiciones del sistema expuestas anteriormente, esta etapa se divide en tres fases: Diseño básico, Optimización y Confrontación.

En la fase de diseño básico se busca un diseño de aplicador de microondas basado en guía de onda que funcione a $2,45 \mathrm{GHz}$, con el cual se pueda conseguir una polarización circular para los dos modos de funcionamiento del sistema, utilizando uno o los dos magnetrones. Como resultado se tiene un diseño básico que debe ser modificado en la fase de optimización con el fin de obtener las condiciones de polarización, adaptación y aislamiento requeridas.

En la fase de optimización debe lograrse un compromiso entre los parámetros de adaptación y asilamiento con la polarización circular requerida en el plano de apertura. Primero se varían las dimensiones del aplicador con el objetivo de minimizar los parámetros de adaptación y aislamiento máximo. Dichas dimensiones son: la longitud de conector interno del coaxial que penetra en la guía de onda, y la distancia entre el corto y la ubicación de la alimentación. Después para asegurar la polarización circular en el plano de apertura se varía la longitud total del aplicador, manteniendo los valores de adaptación y aislamiento sin variaciones significativas.

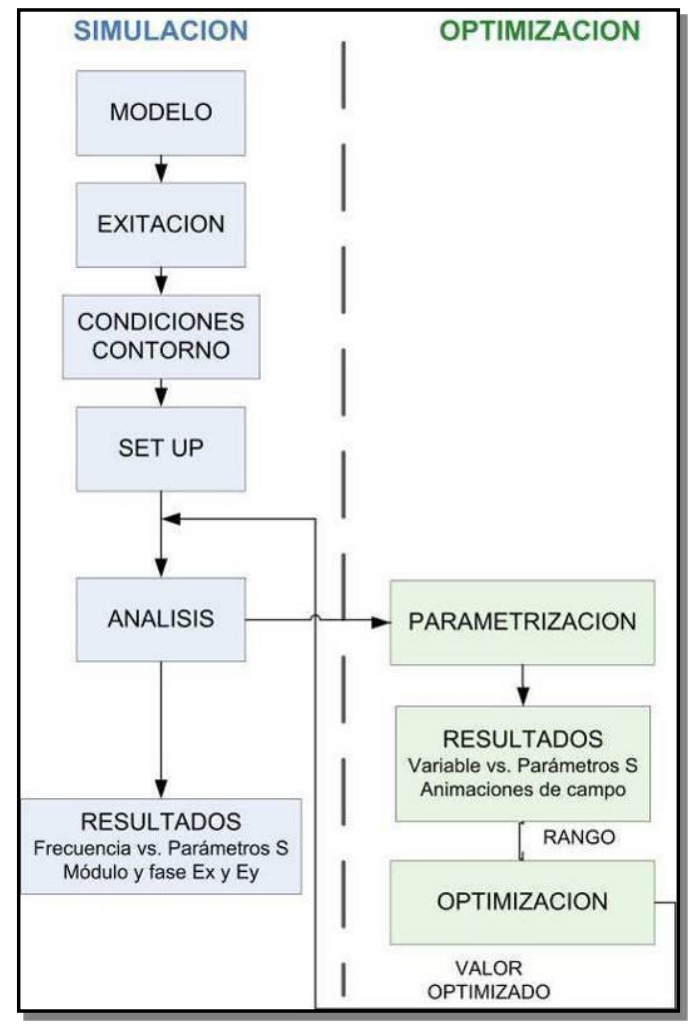

Figura 4-3. Metodología para la optimización 
Los dos puntos anteriores se alcanzan haciendo simulaciones con herramientas que trabajan con elementos finitos (HFSS). En primer lugar se observa la influencia que tiene la longitud del conector sobre los parámetros de scattering, luego se busca la distancia optima a la que se debe colocar la alimentación y por último la longitud más adecuada para garantizar que en el plano de apertura se tiene una polarización circular. El procedimiento seguido en cada simulación de esta fase se muestra en la Figura 4-3.

En el procedimiento, inicialmente se crea el modelo en 3D, se aplican las excitaciones y las condiciones de contorno, se da la frecuencia de funcionamiento y si se desea un barrido de frecuencias, para finalizar con el análisis de la estructura con las dimensiones iníciales. Para continuar con la optimización, se hace el análisis de la estructura variando un parámetro dimensional y observando el comportamiento para diferentes valores; de este modo se tiene el rango alrededor del mínimo de de los parámetros de scattering donde se realiza la optimización. Una vez se tiene el valor óptimo se hace de nuevo el análisis de la estructura y se obtienen los resultados, si estos cumplen con las condiciones planteadas se construye el modelo y si no, se modifican otros parámetros hasta lograr el objetivo.

La polarización circular se confirma obteniendo una relación axial cercana a 1 (en lineal) y un desfase de $0,5 \pi$ entre las componentes del campo Eléctrico en $\mathrm{X}$ y $\mathrm{Y}$; adicionalmente los parámetros de adaptación y aislamiento máximo deben ser mínimos, utilizando para el análisis graficas y animaciones en 2D. Como resultado de esta fase se tiene un modelo inicial que cumpla con las anteriores condiciones.

En la fase de confrontación se confirma que el modelo inicial cumpla con las especificaciones requeridas por la empresa (DS3D S.L.), se adapte a las necesidades del constructor y sea de fácil integración con las demás partes del sistema. En caso contrario se deben hacer las mejoras y ajustes necesarios antes de entrar a la etapa de construcción del prototipo.

En la etapa de construcción del prototipo se construye el diseño definitivo del aplicador (se lleva a cabo en una empresa fuera del grupo GEA) y se integra la parte de la alimentación y control ${ }^{5}$ y el brazo articulado ${ }^{6}$. Con el prototipo construido, se lleva a cabo la etapa de demostración.

En la fase de comprobación lo primero es medir los parámetros de adaptación y comparar los valores con los obtenidos en las simulaciones, para estas medidas se coloca el aplicador sobre una muestra de madera de $10 \mathrm{~cm}$.

En segundo lugar se comprueba que la polarización sea circular en ambos modos de funcionamiento, los materiales usados para este experimento son: un analizador de redes, una bocina con polarización lineal que funciona de 1 hasta $18 \mathrm{GHz}$, el prototipo construido y un spliter tipo $\mathrm{N}$ 1:2 que permite observar el modo de funcionamiento $\mathrm{A}(2 \mathrm{~kW})$.

${ }^{5}$ Desarrollada por otros miembros del grupo GEA

${ }^{6}$ No se ha desarrollado aun, será integrado en etapas posteriores.

$78 \quad$ Tecnología de microondas en el sector de restauración de bienes culturales 


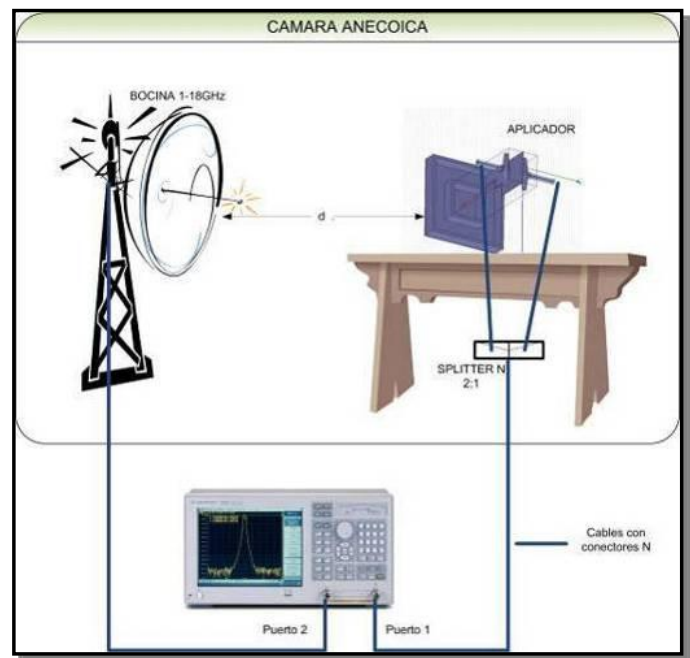

Figura 4-4. Configuración para medidas de polarización

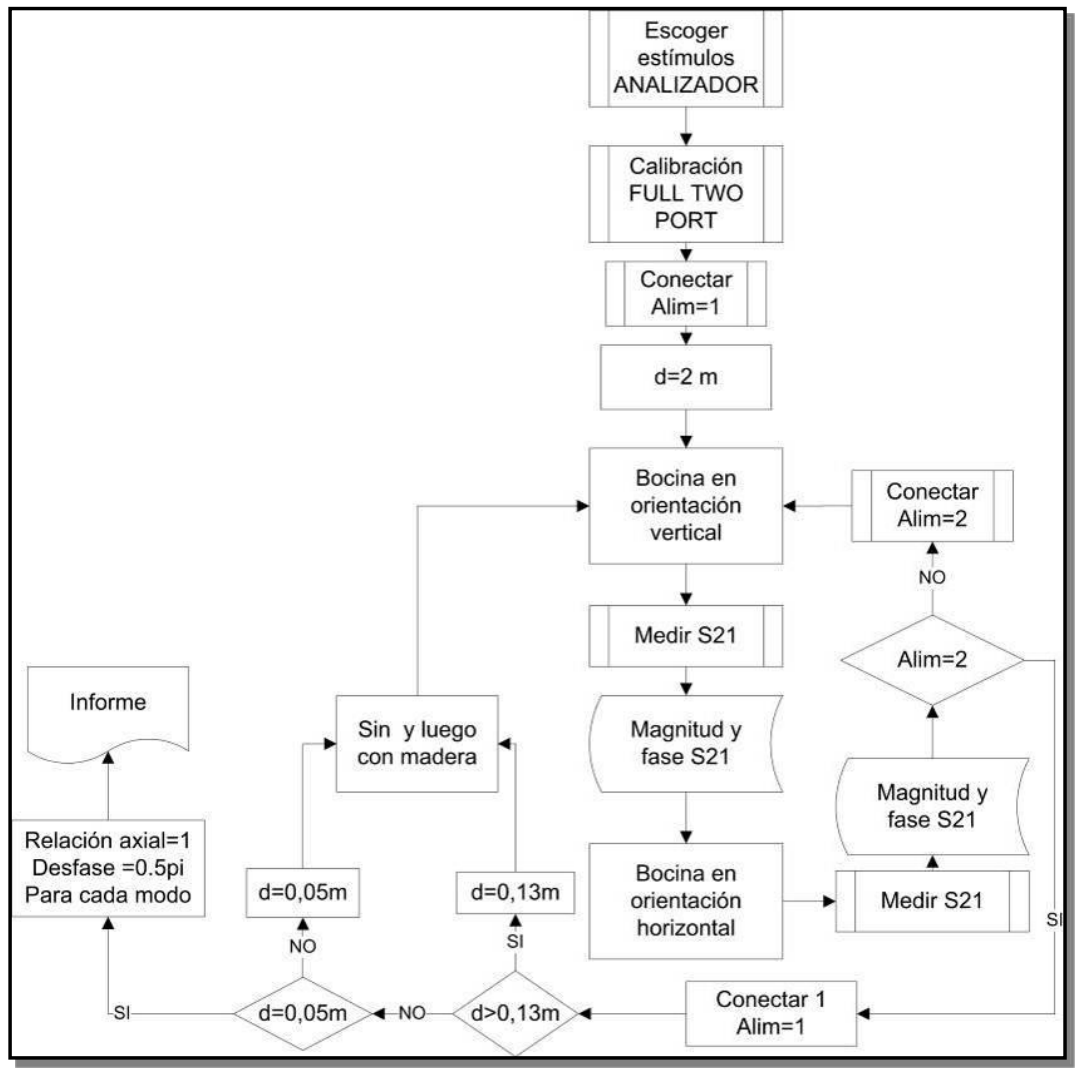

Figura 4-5. Protocolo medida de Polarización 
La configuración del laboratorio se muestra en la Figura 4-4. El objetivo es verificar que la relación axial sea 1 y el desfase de $0,5 \pi$ para cada modo de funcionamiento, para esto se mide el parámetro de transmisión tanto en polarización vertical como horizontal para cada modo y a diferentes distancias del plano de apertura con y sin madera en el trayecto, el procedimiento empleado se muestra en la Figura 4-5.

Por último, se deben conocer los niveles de campo radiado alrededor del aplicador para establecer si representan o no peligro para el ser humano. Adicionalmente se verifica la eficacia del traje apantallado con el que se cuenta.

La Figura 4-6 muestra la configuración del laboratorio, el campo eléctrico es medido mediante un equipo de medida de radiación (EMR) y una sonda de medida de campo eléctrico. Primero se mide la magnitud de campo radiado en el plano paralelo a la superficie irradiada (Figura 4-6 a), localizando el EMR a cuatro alturas diferentes: $0,100,150$ y $175 \mathrm{~cm}$ y a $2,5 \mathrm{~m}$ de distancia desde el centro de la mesa, los datos son tomados para cada altura en saltos $30^{\circ}$ de giro de la mesa.

Luego se mide la magnitud de campo en los cortes principales del diagrama de radiación ${ }^{7}$ (Figura 4-6 b), para esto se localiza el EMR a $2 \mathrm{~m}$ del centro de la mesa en línea de vista con el aplicador, se toman los datos en saltos de $45^{\circ}$ de giro de la mesa, esto se repite para una altura de $50 \mathrm{~cm}$ sobre la línea de vista y $50 \mathrm{~cm}$ debajo de la línea de vista.

El mismo procedimiento se repite para la altura de los genitales, el pecho y la cabeza de un maniquí de $1,85 \mathrm{~cm}$, tanto en el caso que lleve traje como si no lo lleva, con el fin de observar la eficacia del traje apantallado. Para terminar, acercamos el maniquí $50 \mathrm{~cm}$ hacia el aplicador y medimos el campo con traje a la altura de la cadera, con el objetivo de verificar el aumento de potencia en función de la distancia.

En la fase de medidas se mide la temperatura sobre muestras de madera con la configuración del laboratorio de la Figura 3-1. Se hacen dos experimentos: medida de temperatura con aplicación central y solapada para 5 tablas radiales, 5 tangenciales y 5 tangenciales jóvenes. La ubicación de las sondas es la mostrada en la Figura 4-7, el procedimiento se resume en la Figura 4-8 y el tratamiento se hace en el modo de funcionamiento B $(1 \mathrm{~kW})$.

${ }^{7}$ En la práctica se utiliza un solo corte porque se tiene polarización circular.

80 Tecnología de microondas en el sector de restauración de bienes culturales 


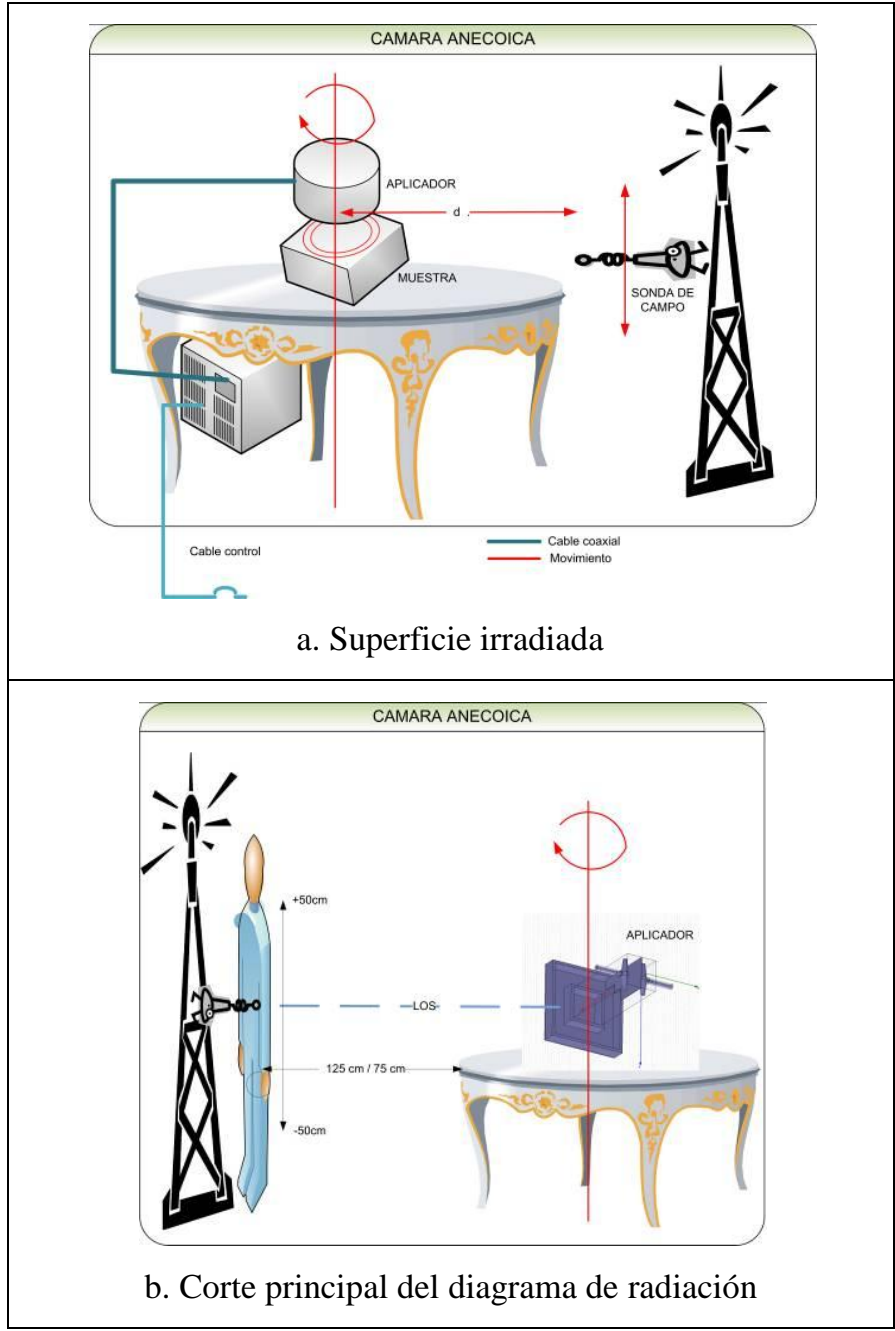

Figura 4-6. Configuración medida de campo

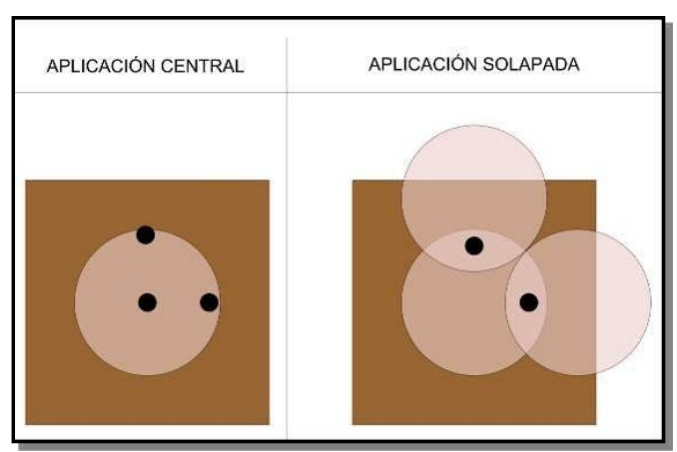

Figura 4-7. Ubicación sondas de temperatura 


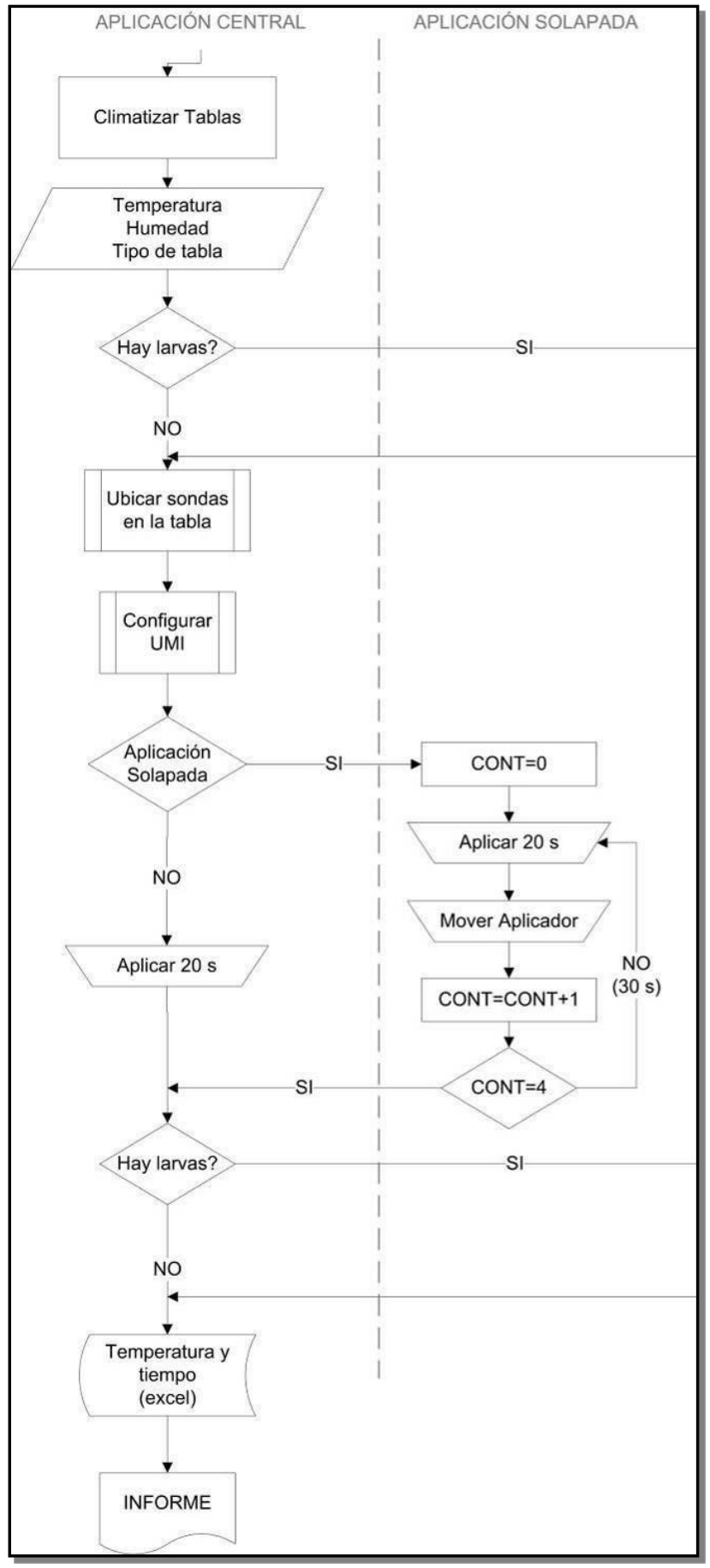

Figura 4-8.Procedimiento experimental medidas de temperatura 


\subsection{DISEÑO BÁSICO}

El punto de partida es una guía de onda cuadrada de $75 \times 75 \mathrm{~mm}$. Esta guía se alimenta con dos coaxiales, cada uno de los cuales adquiere la potencia de microondas de un magnetrón.

La polarización circular se consigue ubicando un septum en la mitad de la guía de onda cuadrada, formando dos guías de onda rectangulares. Para el modo de funcionamiento A se activan los dos magnetrones formando una red de alimentación que combina sus potencias; y para el modo de funcionamiento B se activa uno de los dos magnetrones, de esta manera el septum funciona como un conversor de polarización

Adicionalmente, se cuenta con una trampa de ondas en el plano de la apertura del aplicador para que la potencia radiada hacia atrás sea mínima y hacer más directivo el haz de microondas entregado. También se prevé la necesidad de un radomo de teflón. Las características iniciales de este diseño son:

- Longitud total de 264,5 mm.

- Distancia desde el corto hasta la alimentación de $37 \mathrm{~mm}$.

- Longitud del conector interior dentro de la guía de $25 \mathrm{~mm}$

- Distancia desde el final del septum al plano de apertura de $106 \mathrm{~mm}$.

- Coaxial RG58, diámetro interno de $0,8 \mathrm{~mm}$ y diámetro externo de $3 \mathrm{~mm}$.

La longitud de onda para cada una de las guías de onda rectangulares es:

$$
\begin{aligned}
& \lambda_{0}=\frac{3 \times 10^{8}}{2,45 \times 10^{9}}=122 \mathrm{~mm} \\
& \lambda_{0}=2 \times 75 \mathrm{~mm}=150 \mathrm{~mm} \\
& \lambda_{g}=\frac{\lambda_{0}}{\sqrt{1-\left(\frac{\lambda_{0}}{\lambda_{c}}\right)^{2}}}=209,7 \mathrm{~mm}
\end{aligned}
$$

Se hace una simulación para cada uno de los modos de funcionamiento, con los resultados se realiza la optimización del siguiente apartado para el modo de funcionamiento A, luego se hacen las modificaciones en el modo de funcionamiento $\mathrm{B}$, para confirmar que también se tiene polarización circular. El diseño básico se muestra en la Figura 4-9. 


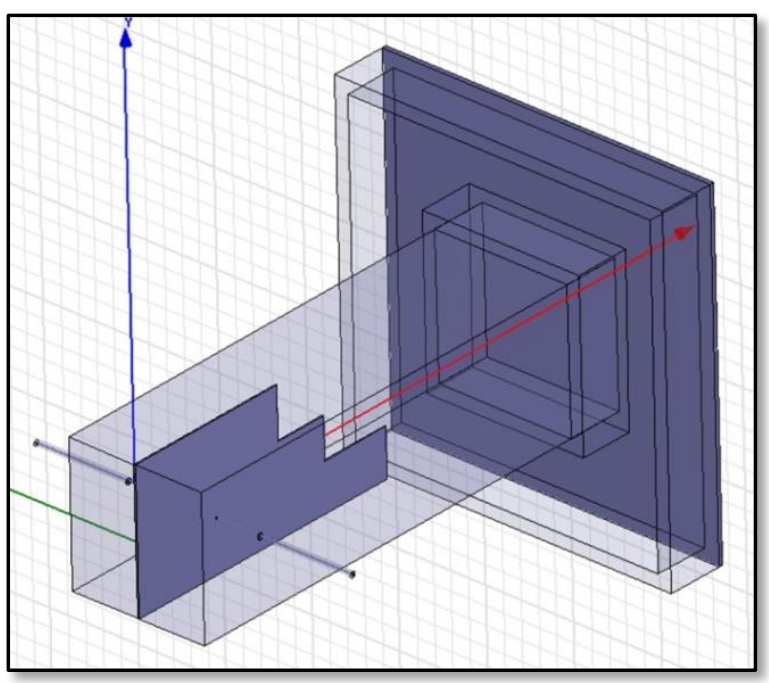

Figura 4-9. Diseño Básico

\subsubsection{Modo de funcionamiento $A$}

En la Figura 4-10 se sintetizan los parámetros a evaluar para la polarización circular, estos se han calculado a partir del valor de magnitud y fase del campo eléctrico tanto en el eje X como en él Y. Los datos representados son los del eje central del lóbulo de radiación; en la gráfica el cero representa la cara de radiación y los demás puntos son cinco planos ubicados a $2 \mathrm{~cm}$ del plano de apertura y distanciados $\lambda \mathrm{o} / 10$ entre sí. Las graficas de campo se pueden consultar en el Apéndice IV.

Los parámetros de Scattering se muestran en la Figura 4-11. En (a) el valor de los parámetros de reflexión a $2,45 \mathrm{GHz}$ es $-8,81 \mathrm{~dB}$, tanto $\mathrm{S}_{11}$ (en azul) como el $\mathrm{S}_{22}$ (en rojo); en (b) el valor de los parámetros de aislamiento es -16,62 dB, iguales en todo el rango por ser una red recíproca.

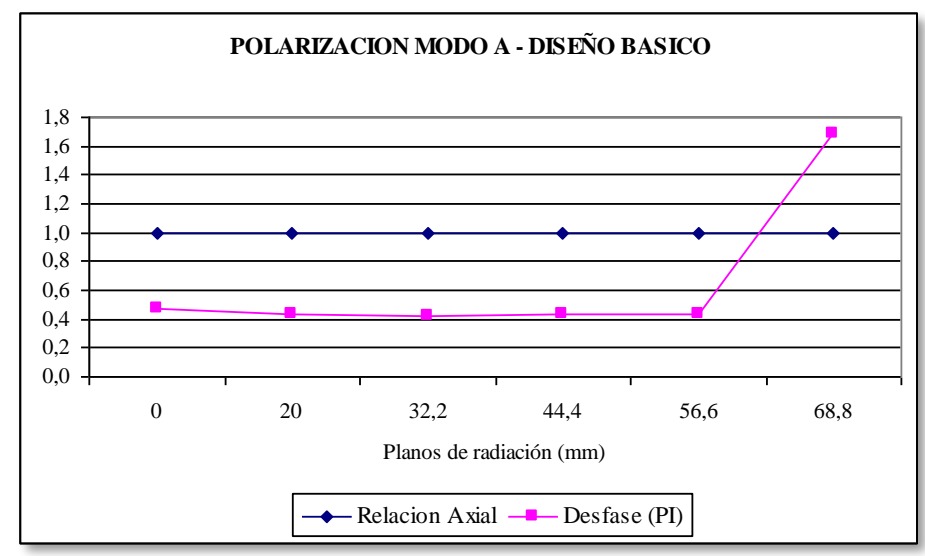

Figura 4-10. Parámetros Polarización modo A - Diseño Básico

$84 \quad$ Tecnología de microondas en el sector de restauración de bienes culturales 


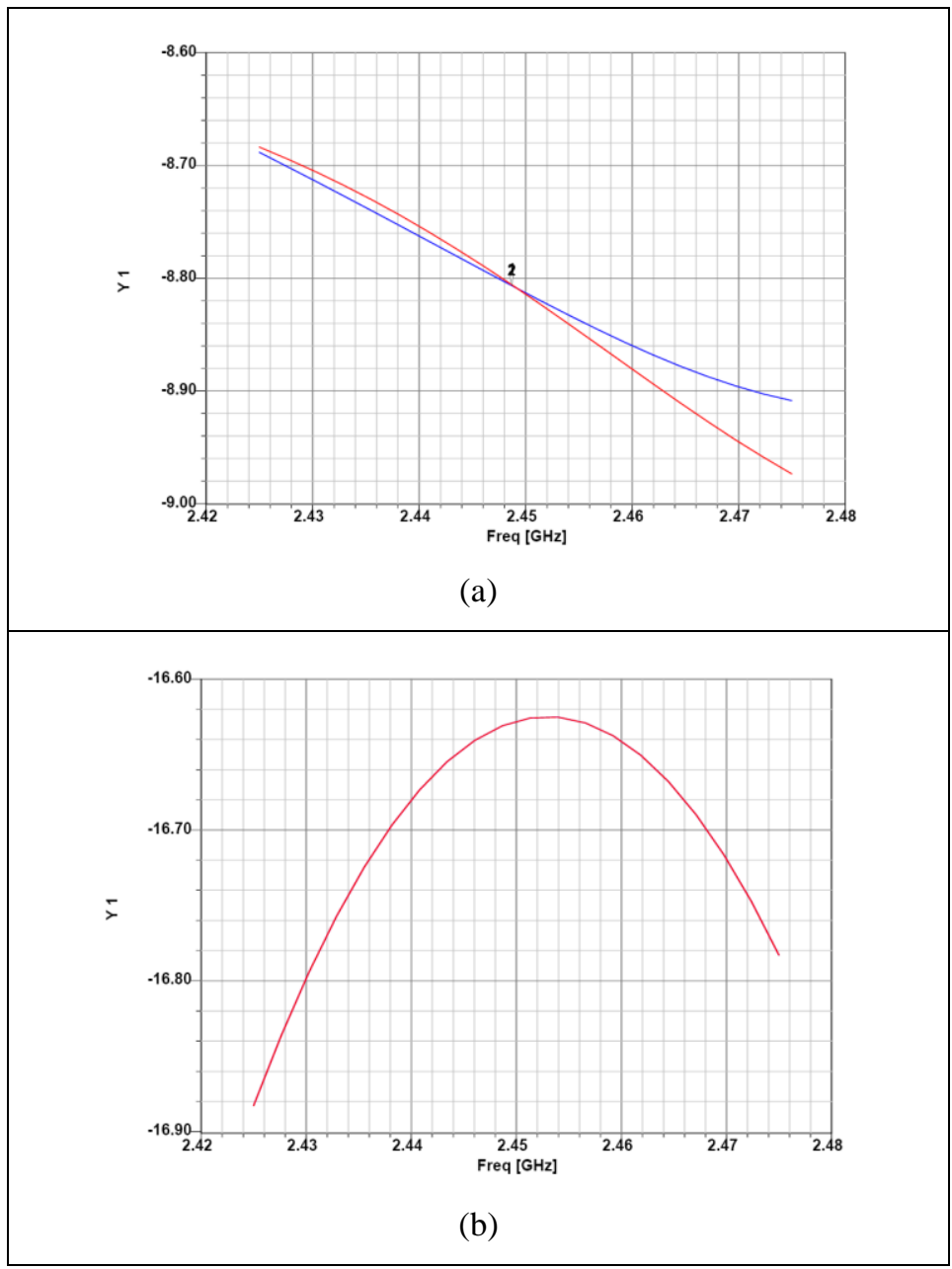

Figura 4-11. Parámetros S modo A- Diseño Básico

En conclusión, el diseño básico para el modo A presenta un excelente comportamiento en polarización hasta a $5,5 \mathrm{~cm}$ del plano de apertura en espacio abierto, en cuanto a los parámetros $\mathrm{S}$ los valores son buenos pero se pueden mejorar en el apartado de optimización.

\subsubsection{Modo de funcionamiento $B$}

En la Figura 4-12 se muestran los parámetros para la polarización circular, calculados bajo los mismos criterios que en el modo de funcionamiento A. Las graficas de campo se pueden consultar en el Apéndice IV. 


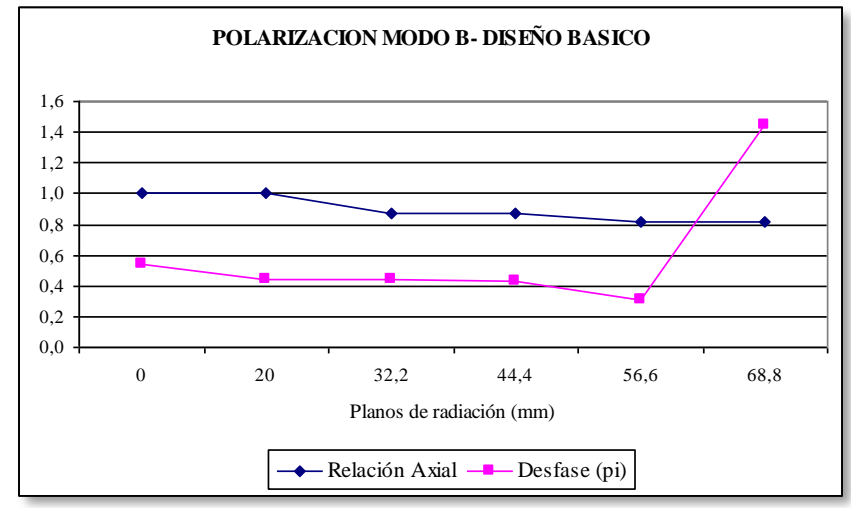

Figura 4-12. Parámetros polarización modo B - Diseño Básico

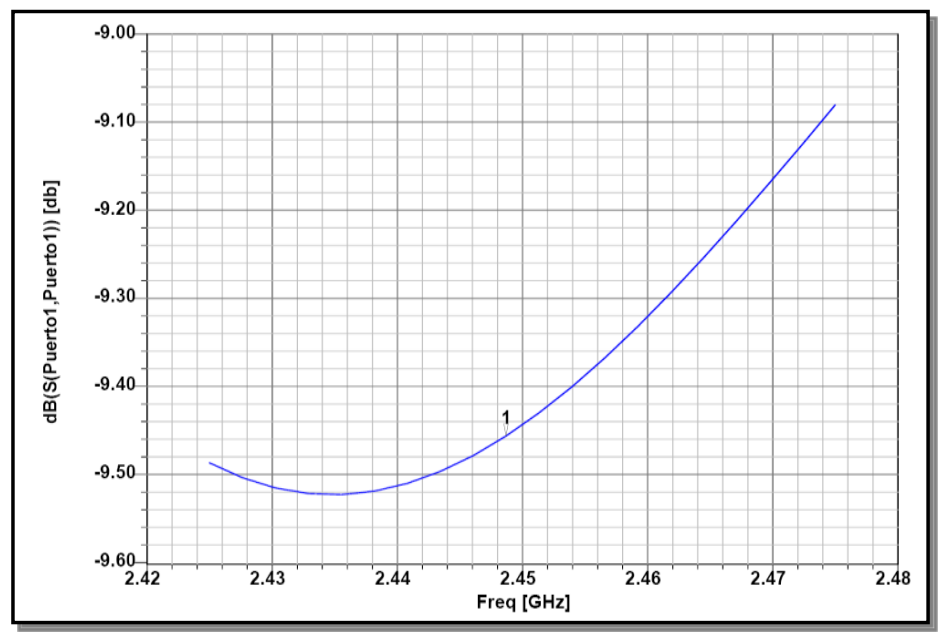

Figura 4-13. Parámetros S modo B- Diseño Básico

El parámetro $S_{11}$ se muestra en la Figura 4-13, cuyo valor es $-9,45 \mathrm{~dB}$. En conclusión, el diseño básico para el modo B presenta un buen comportamiento en polarización para el plano de apertura, estas condiciones se degradan a medida que nos alejamos de dicha cara, en cuanto al $S_{11}$ su valor es bueno pero se puede mejorar en el siguiente apartado.

\subsection{OPTIMIZACIÓN}

El objetivo de este apartado es mejorar los parámetros $\mathrm{S}$ del diseño básico, mientras se mantiene la polarización circular en el plano de apertura. Para ello se tienen en cuenta dos aspectos: primero la longitud del conector interno que entra en la guía y segundo la distancia entre la ubicación de la alimentación y el corto circuito que hay al final de la guía.

Una vez estos parámetros son optimizados se varía la longitud de la guía con el fin de asegurar las mejores características posibles de polarización circular en el plano 
de apertura. Por último, es importante tener en cuenta aquellos detalles que puedan ayudar al buen funcionamiento del aplicador una vez construido.

La metodología en los siguientes sub-apartados es hacer la optimización para el modo de funcionamiento A y verificar que se cumplan las condiciones deseadas en el modo de funcionamiento B.

\subsubsection{Longitud del conector interno}

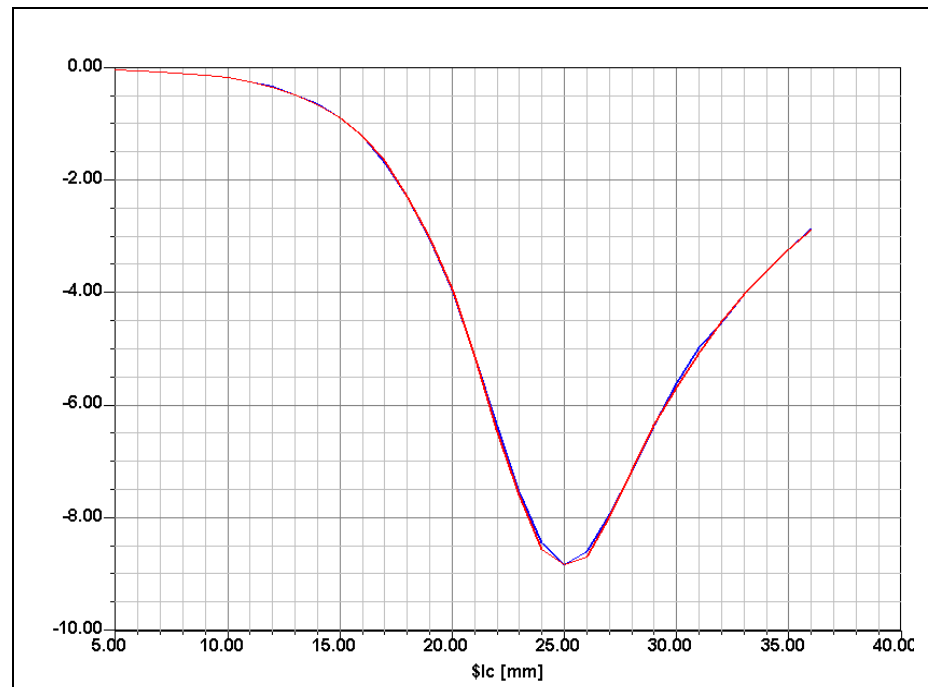

(a)

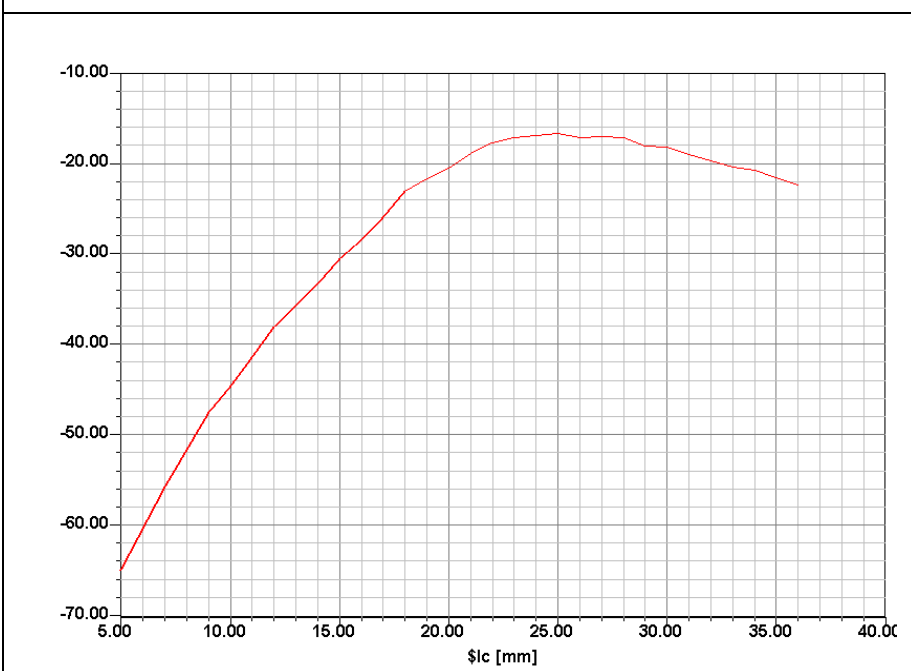

(b)

Figura 4-14. Parametrización longitud del conector interno 
Con las dimensiones descritas en el diseño básico se varía la longitud del conector interno desde 5 hasta $36 \mathrm{~mm}$ en saltos de un milímetro, con el fin de encontrar la longitud en la que los valores de los parámetros $\mathrm{S}$ sean mínimos.

Los resultados de esta parametrización para el modo de funcionamiento A se pueden observar en la Figura 4-14, donde en (a) el valor mínimo de $\mathrm{S}_{11}$ es -8,86 dB para una profundidad de $25 \mathrm{~mm}$, en (b) a $25 \mathrm{~mm}$ el valor del aislamiento es -16,77 dB. Aunque en este último caso no es el valor mínimo, el valor es aceptable ya que mantiene un compromiso con los resultados de los parámetros de reflexión.

En conclusión, se considera que la longitud del conector interno más apropiada para las restantes simulaciones es $25 \mathrm{~mm}$, tanto para el modo de funcionamiento A como para el B, aunque no se hagan simulaciones para el segundo caso.

\subsubsection{Ubicación de la alimentación}

La distancia teórica a la que se debe ubicar este tipo de alimentación es a $\lambda \mathrm{g} / 4$ [45] desde la pared eléctrica de la guía, así que se hace una parametrización inicial entre $\lambda \mathrm{g} / 4 \pm \lambda \mathrm{g} / 8$. Dicha parametrización tiene 18 puntos de evaluación comprendidos entre 28,4 y 76,4 mm, en saltos de $3 \mathrm{~mm}$ (diámetro exterior del RG58).

Los resultados obtenidos para el modo de funcionamiento A pueden verse en la grafica de la Figura 4-15, de donde se puede afirmar que no se ve el mínimo del parámetro $S_{11}$ (azul) ni del $S_{22}$ (magenta), por esta razón se decide ampliar el rango de parametrización para observar el comportamiento a menores distancias desde la pared eléctrica. Por otro lado, en las animaciones 1 y 2 los valores de la relación axial y el desfase en el plano de apertura son correctos y homogéneos hasta una distancia de la alimentación desde la pared de corto de 52,4 mm.

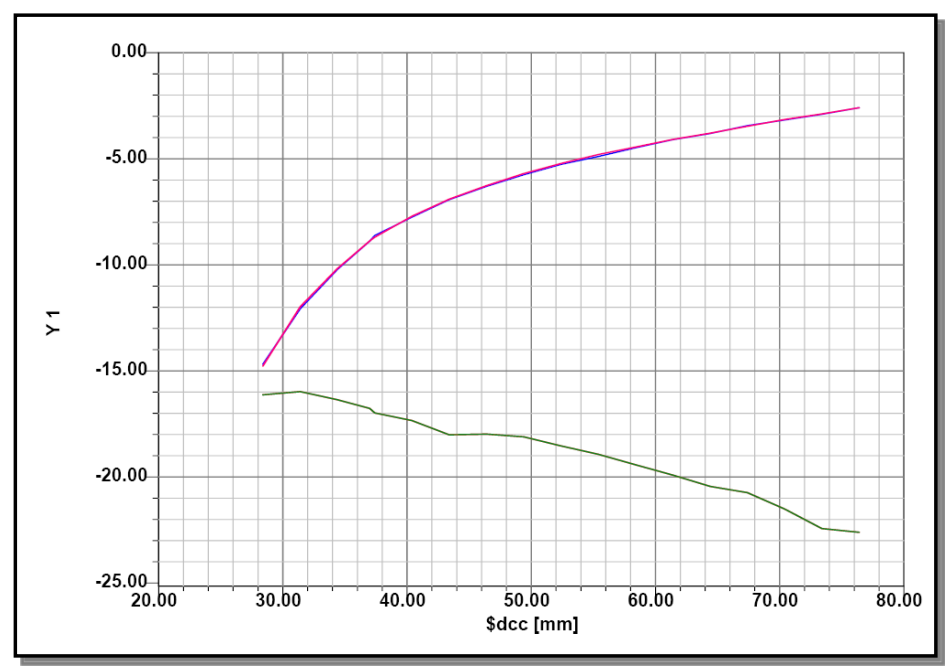

Figura 4-15. Parametrización Inicial - ubicación alimentación 
La nueva parametrización está hecha desde 13,4 hasta 52,4 mm en saltos de $3 \mathrm{~mm}$. La Figura 4-16 muestra los resultados, el mínimo para los parámetros de aislamiento se sitúa alrededor de $24 \mathrm{~mm}$, por lo que la optimización se plantea para el rango en el que el valor es inferior a $-10 \mathrm{~dB}$, es decir, entre 16 y $40 \mathrm{~mm}$. En cuanto a la polarización circular las características en el plano de apertura siguen siendo las deseadas.

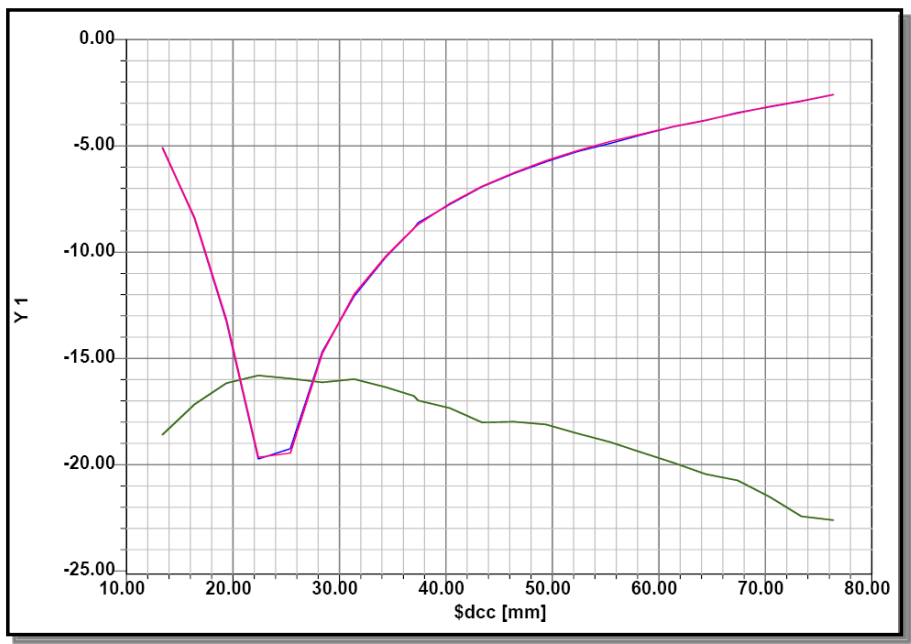

Figura 4-16. Parametrización - ubicación alimentación

En la Figura 4-17 se muestran parámetros $\mathrm{S}$ obtenidos después de la optimización (entre 16 y $40 \mathrm{~mm}$ ), cuya función de coste está definida para encontrar sus valores mínimos. El resultado es ubicar la alimentación a 23,82 $\mathrm{mm}$ de la pared eléctrica de la guía, donde el $S_{11}$ (azul) y $S_{22}$ (magenta) son $-20,70 \mathrm{~dB}$ y el $S_{12}$ (rojo) y $S_{21}$ (verde) son $-15,56 \mathrm{~dB}$.

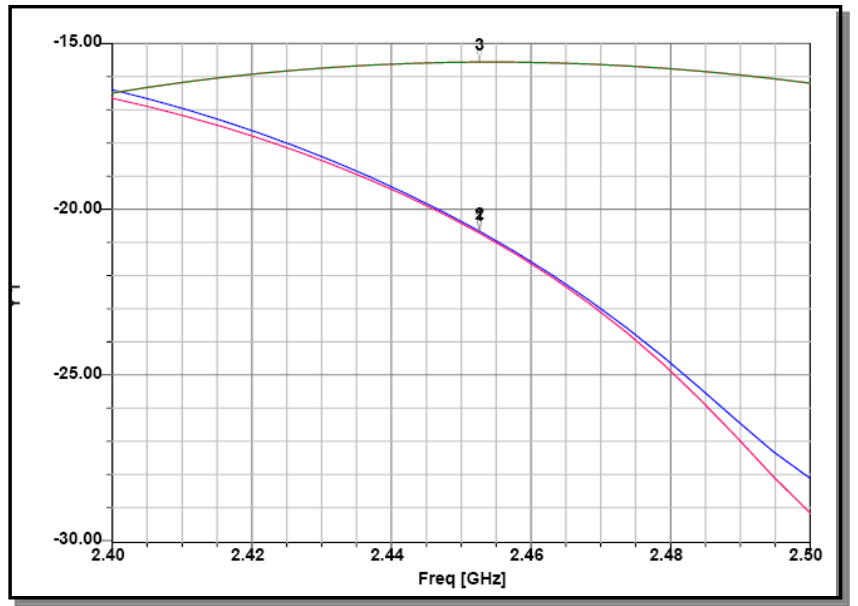

Figura 4-17. Optimización ubicación alimentación - Modo A 
En relación con la polarización circular en el plano de apertura, se puede ver que la relación axial y el desfase tienen los valores deseados, 1 y $0,5 \pi$ respectivamente, en las Figura 4-18 y Figura 4-19.

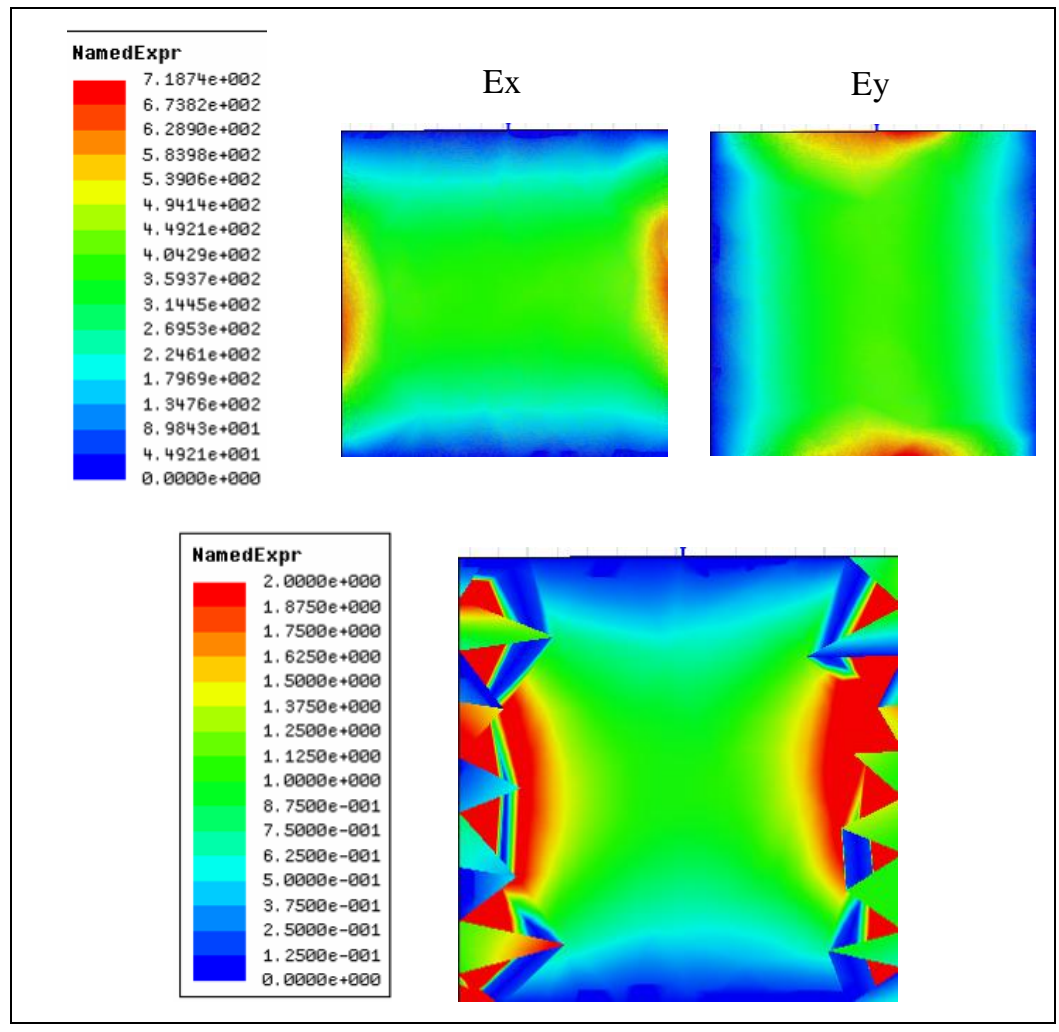

Figura 4-18. Relación Axial Ubicación alimentación-Modo A 


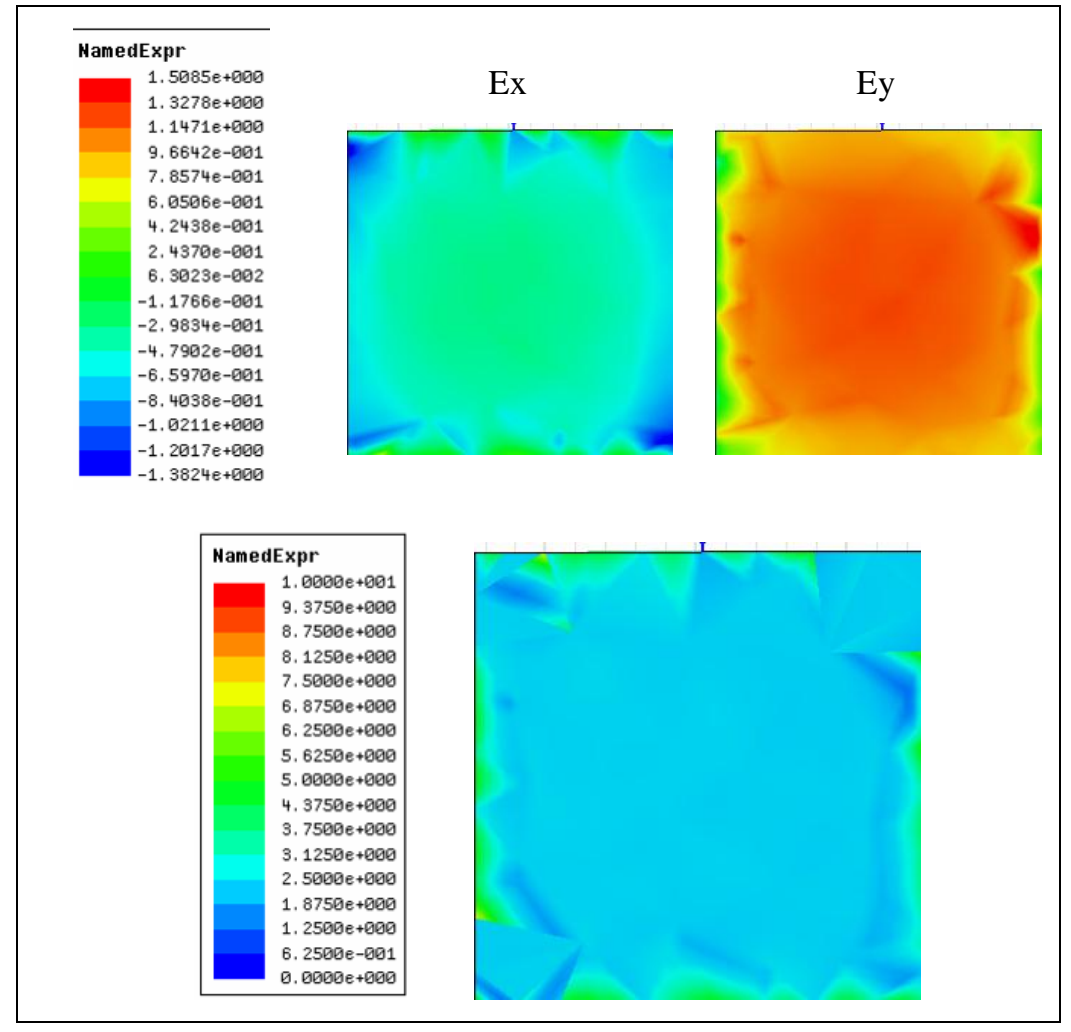

Figura 4-19. Desfase Ubicación alimentación - Modo A

Siguiendo la metodología planteada, el resultado obtenido se aplica al modo de funcionamiento $\mathrm{B}$; de este modo ubicando la alimentación a 23,82 $\mathrm{mm}$ desde la pared eléctrica, el parámetro $\mathrm{S}_{11}$ a $2,45 \mathrm{GHz}$ se reduce menos de $1 \mathrm{~dB}$ pasando a tener un valor de $-19,96 \mathrm{~dB}$.

La relación axial y el desfase en el plano de apertura después de la modificación se muestran en las Figura 4-20 y Figura 4-21 y se mantienen las condiciones de polarización circular planteadas.

En resumen, los resultados obtenidos con la alimentación ubicada a 23,82 mm del corto para ambos tipos de funcionamiento son los correctos tanto en adaptación donde los parámetros $\mathrm{S}$ están por debajo de los $-15 \mathrm{~dB}$, como en polarización donde se cumplen las dos condiciones evaluadas de relación axial y desfase. 


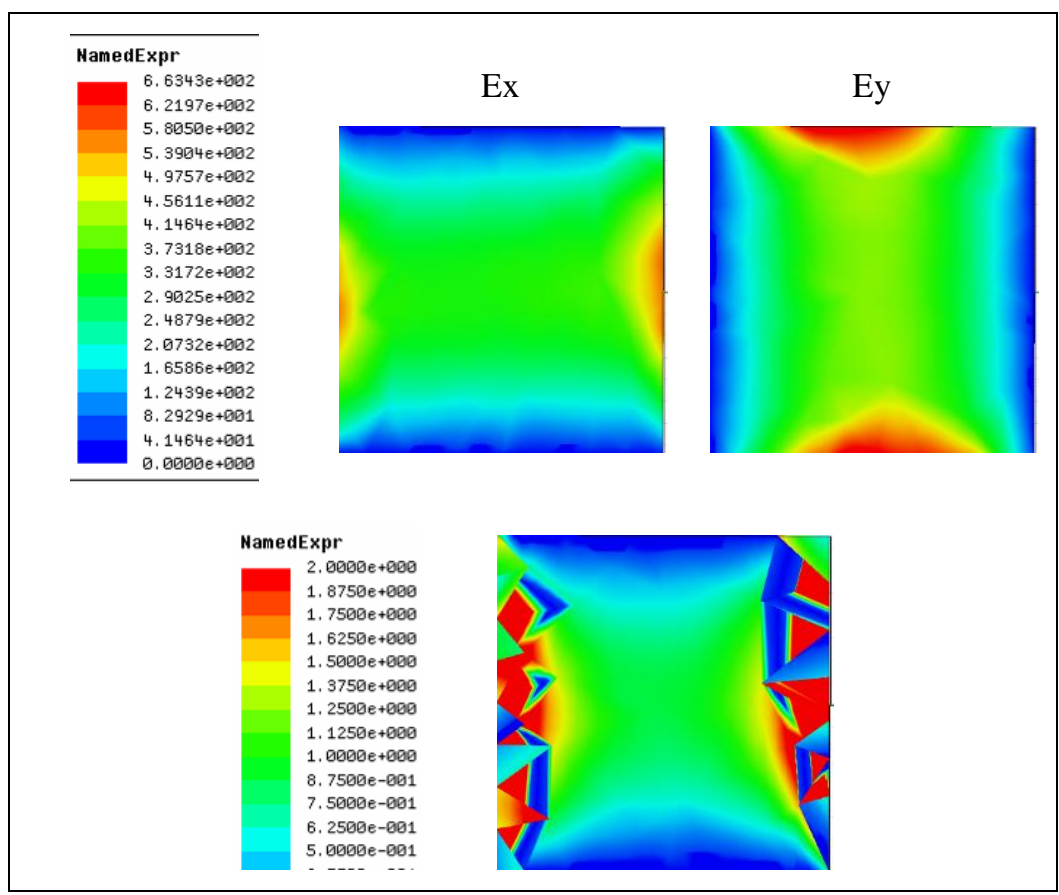

Figura 4-20. Relación Axial ubicación alimentación - Modo B

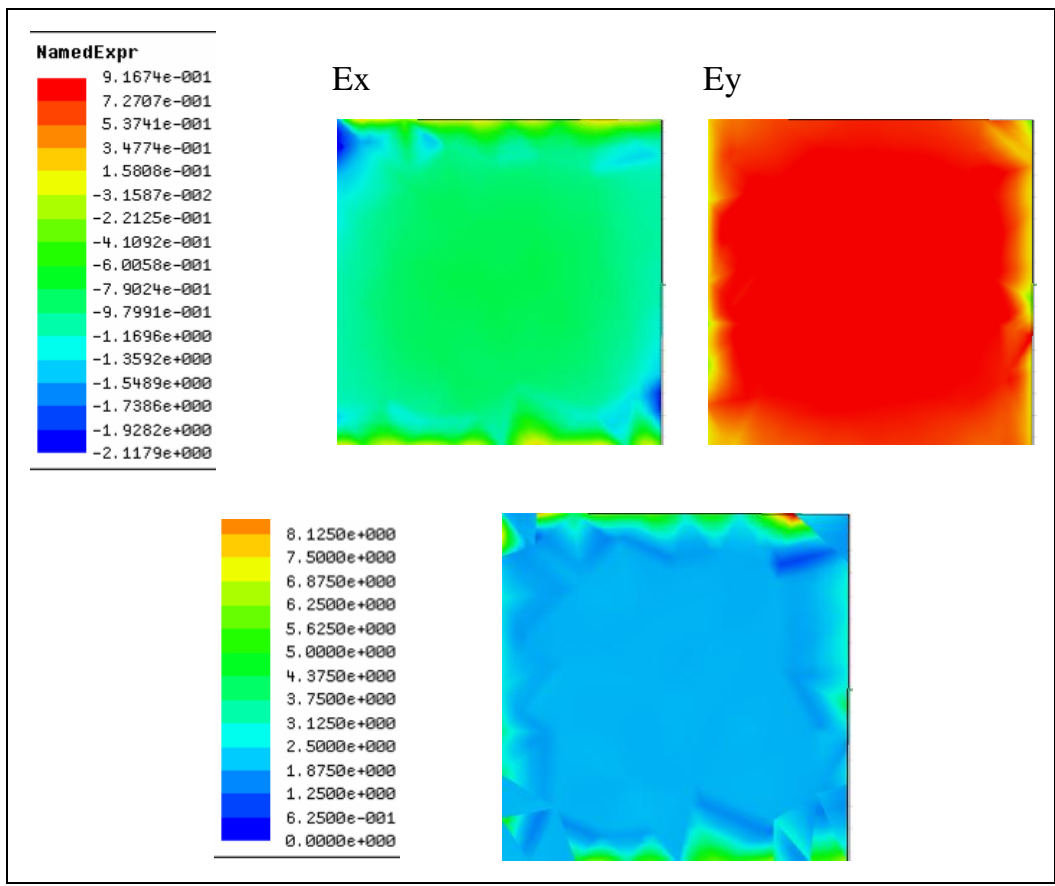

Figura 4-21. Desfase ubicación alimentación - Modo B 


\subsubsection{Ajustes para la construcción}

Debido a la gran magnitud de campo eléctrico que se observa sobre la punta del coaxial en las simulaciones del apartado 0 ., se conecta el conector interno a las paredes de la guía mediante una estructura metálica (Figura 4-22), con el fin de evitar el efecto de puntas. Para lograr esto es necesario cambiar los diámetros del coaxial debido al mecanizado y los tornillos, el diámetro interno es de $7 \mathrm{~mm}$ y el externo de $16 \mathrm{~mm}$.

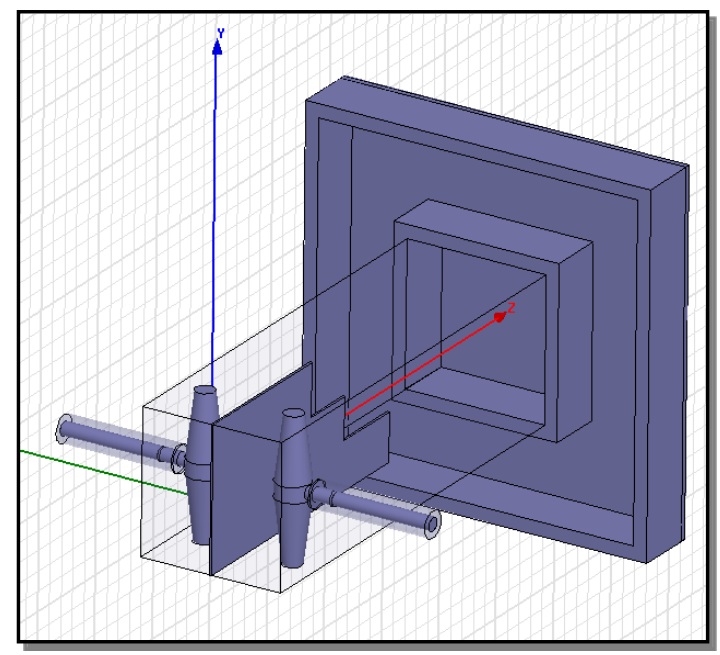

Figura 4-22. Diseño Optimizado con transición

Teniendo en cuenta la ubicación de dicha estructura metálica o transición en una guía estándar WR340, se ubica en una guía de $75 \times 37,5 \mathrm{~mm}$, la distancia a corto inicial es $32,12 \mathrm{~mm}$, la longitud del conector interno se mantiene en $25 \mathrm{~mm}$ y se mide desde la pared de la cavidad hasta el centro de la transición.

Los resultados de los parámetros $\mathrm{S}$ con la introducción de la transición se muestran en la Figura 4-23, dichos resultados para una frecuencia de 2,45 GHz son: $\mathrm{S}_{11}$ (azul) $-6,85 \mathrm{~dB}, \mathrm{~S}_{22}$ (rojo) -6,92 dB y $\mathrm{S}_{12 / 21}-15,56 \mathrm{~dB}$, resultados que en adaptación es necesario optimizar.

En las Figura 4-24 y Figura 4-25 se muestran la relación axial y el desfase obtenidos en la simulación, observando que la polarización circular en el plano de apertura sigue estando presente, aunque puede buscarse una mayor homogeneidad. 


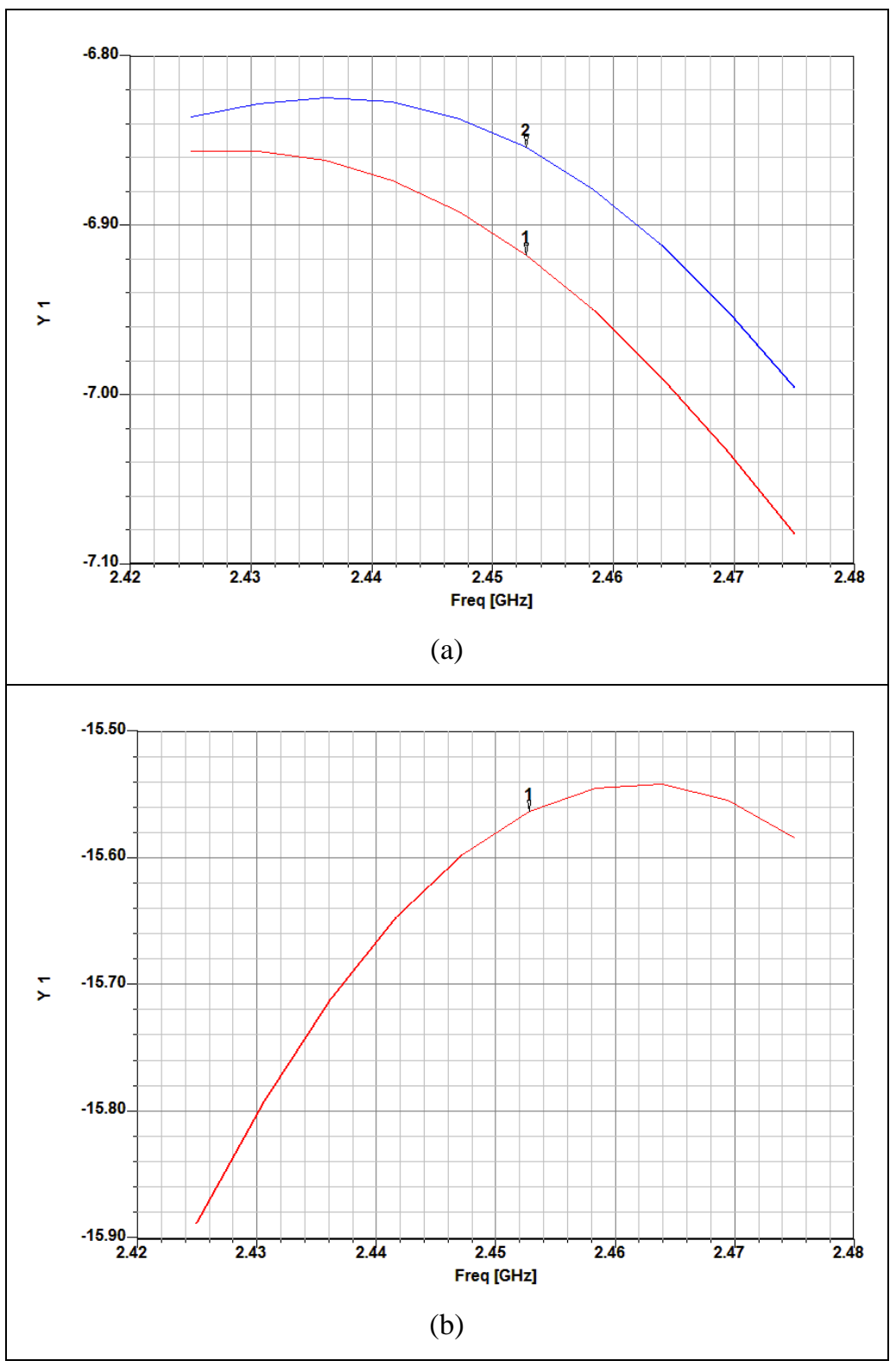

Figura 4-23. Parámetros $S$ con transición - Modo A 
NamedExpr

\begin{tabular}{|c|}
\hline $5.5282 e+002$ \\
\hline 5. $1827 e+002$ \\
\hline $4.8371 e+002$ \\
\hline 4. $4916 e+002$ \\
\hline $4.1461 e+002$ \\
\hline 3. $8006 e+002$ \\
\hline 3. $4551 e+002$ \\
\hline $3.1096 e+002$ \\
\hline $2.7641 e+002$ \\
\hline 2. $4186 e+002$ \\
\hline $2.0731 e+002$ \\
\hline 1. $7276 e+002$ \\
\hline 1. $3820 e+002$ \\
\hline 1. $0365 e+002$ \\
\hline 6. $9102 e+001$ \\
\hline 3. $4551 e+001$ \\
\hline $0.0000 e+000$ \\
\hline
\end{tabular}

Ex

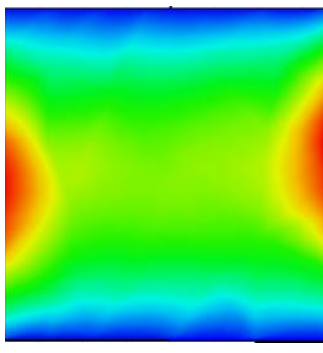

Ey

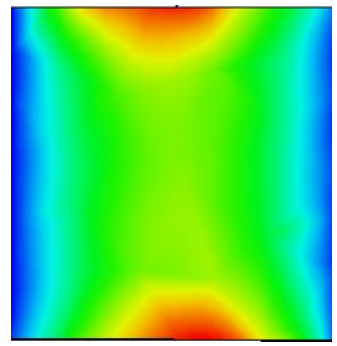

\section{NamedExpr}

$2.0000 e+000$

1. $8750 e+000$

1. $7590 e+000$

1. $6250 e+000$

$1.5000 e+000$

1. $3750 e+000$

1. $2500 e+000$

1. $1250 e+000$

1. $0000 \mathrm{e}+000$

8. $7500 e-001$

7. $5000 e-001$

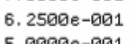

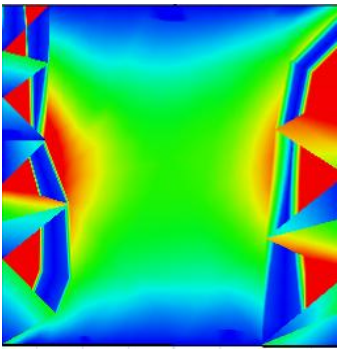

Figura 4-24. Relación axial con transición- Modo A

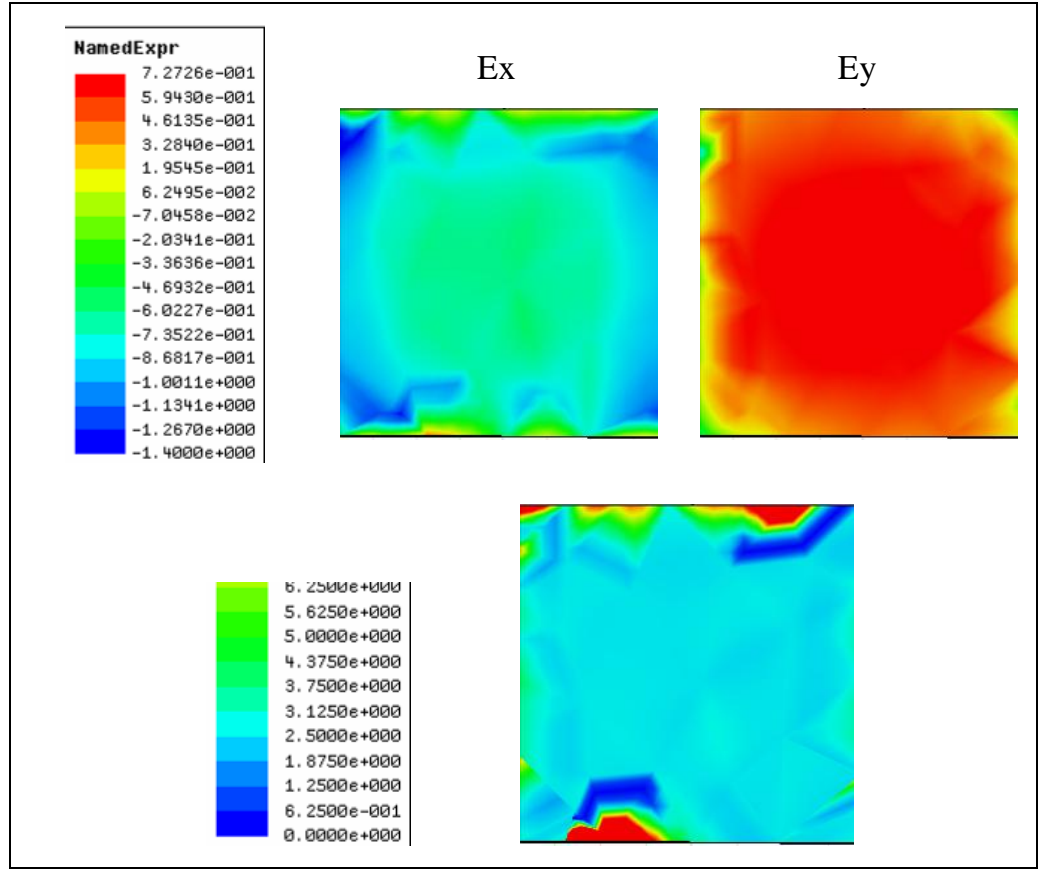

Figura 4-25. Desfase con transición-Modo A 


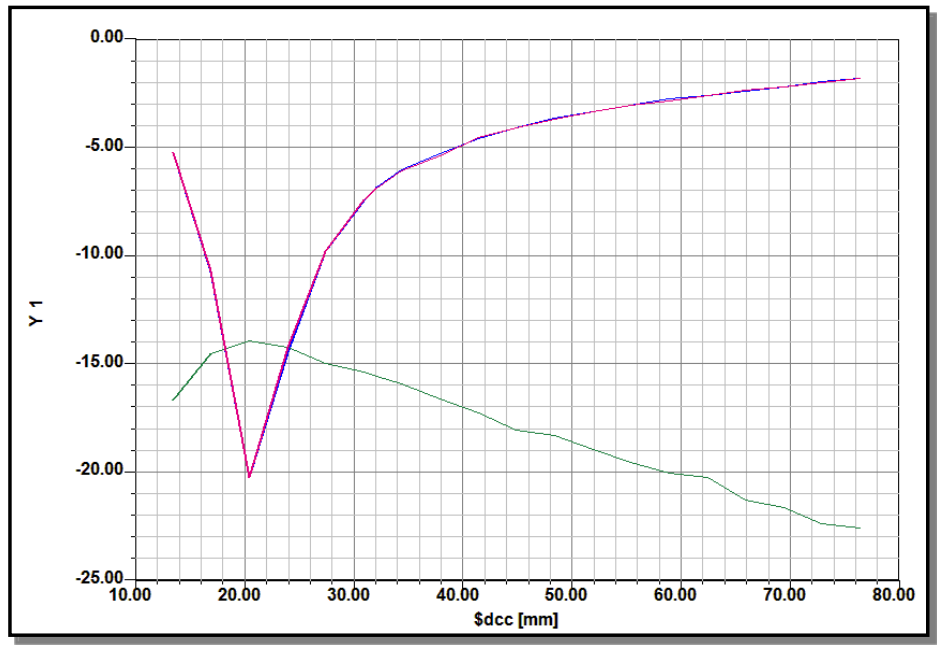

Figura 4-26. Parametrización alimentación con transición-Modo A

En apartados anteriores se ha demostrado que al cambiar la ubicación de la alimentación se logra una mejora sustancial en los parámetros de adaptación, así que se hace una parametrización de esta distancia entre 13,4 y 76,4 $\mathrm{mm}$ en saltos de 3,5 mm (radio interior), los resultados se muestran en la Figura 4-26, de donde se obtiene el rango de optimización entre 15 y $25 \mathrm{~mm}$.

Una vez hecha la optimización en el rango propuesto, el valor de la distancia entre la alimentación y la pared eléctrica es de $20,49 \mathrm{~mm}$, los parámetros de adaptación (Figura 4-27) para esta distancia son: $\mathrm{S}_{11}$ (azul) -20,49 dB, $\mathrm{S}_{22}$ (magenta) -21,16 dB y $\mathrm{S}_{12 / 21}$ (rojo/verde) $-13,82 \mathrm{~dB}$.

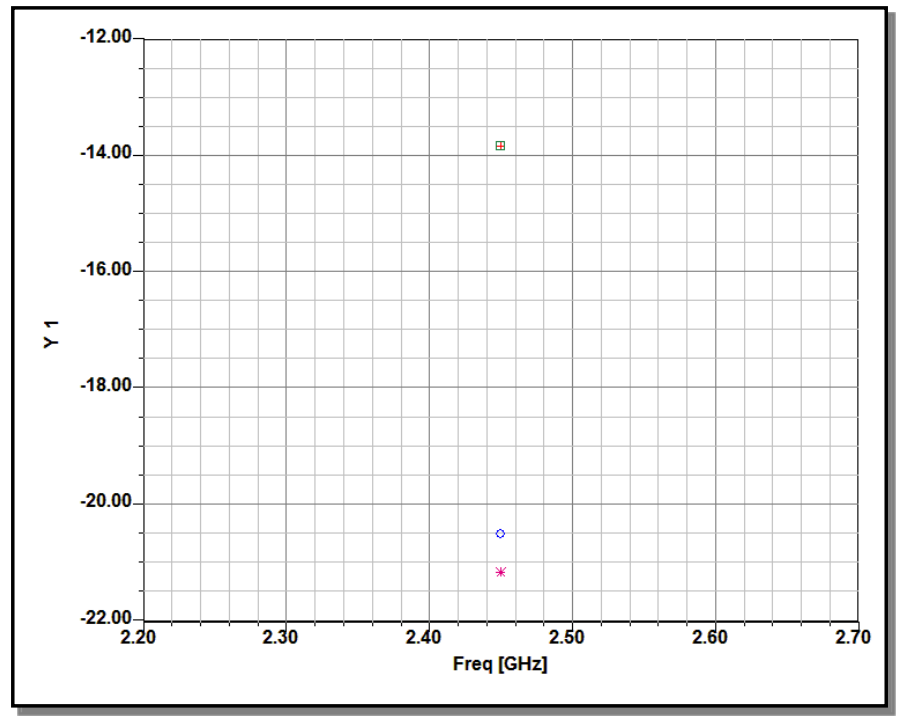

Figura 4-27. Parámetros $S$ con transición optimizada -Modo A 
Ahora solo falta asegurar la polarización circular en el plano de apertura del aplicador, para esto se hace una parametrización de la longitud entre el final del septum y dicho plano comprendida entre 52 y $157 \mathrm{~mm}$ en saltos de $5 \mathrm{~mm}$. Los resultados mostrados en la Figura 4-28 confirman que esta distancia no produce cambios superiores a $\pm 1 \mathrm{~dB}$ en los parámetros $\mathrm{S}$, por lo que no es necesaria una optimización conjunta con los parámetros antes estudiados o una variación de los mismos.

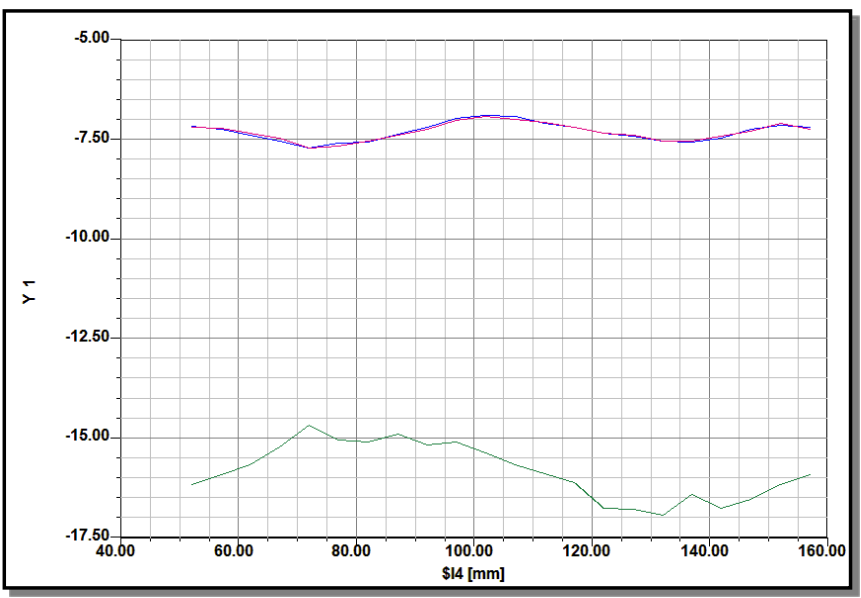

Figura 4-28. Parametrización de la longitud del aplicador - Modo A

En relación con la polarización circular, en las animaciones 3 y 4 puede verse que la relación axial es más homogénea entre 92 y $112 \mathrm{~mm}^{8}$, mientras que el desfase lo es entre 82 y $102 \mathrm{~mm}^{9}$, por lo que la distancia entre el final del septum y el plano de apertura del aplicador debe estar entre 92 y $102 \mathrm{~mm}$. Se considera en base a estos resultados, que una distancia igual a $97 \mathrm{~mm}$ es adecuada para obtener la mejor polarización circular en el plano de apertura.

Una vez obtenidos los valores definitivos de ubicación de alimentación (20,68 mm) y la longitud del aplicador después del septum $(97 \mathrm{~mm})$, se hace una simulación cuyos resultados se resumen en las Figura 4-29, Figura 4-30 y Figura 4-31. Los parámetros de adaptación a $2,45 \mathrm{GHz}$ son: $\mathrm{S}_{11}$ (azul) $-21,24 \mathrm{~dB}, \mathrm{~S}_{22}$ (rojo) -20,9 dB y $\mathrm{S}_{12 / 21}$ (verde) $-13,16 \mathrm{~dB}$.

\footnotetext{
${ }^{8}$ puntos $9-13$.

${ }^{9}$ puntos 7-11.
} 


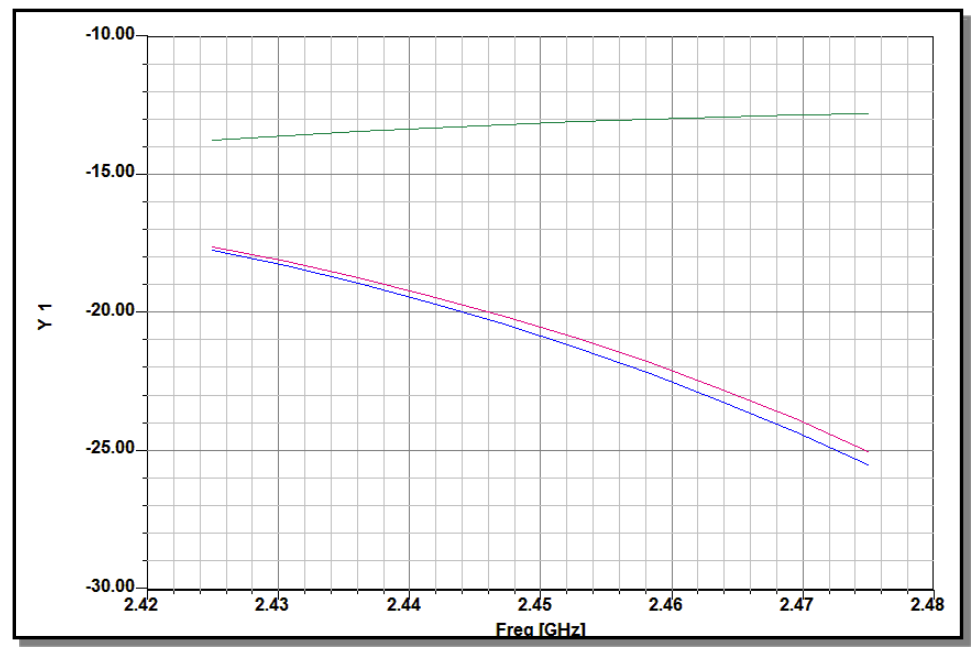

Figura 4-29. Parámetros S diseño ajustado - Modo A

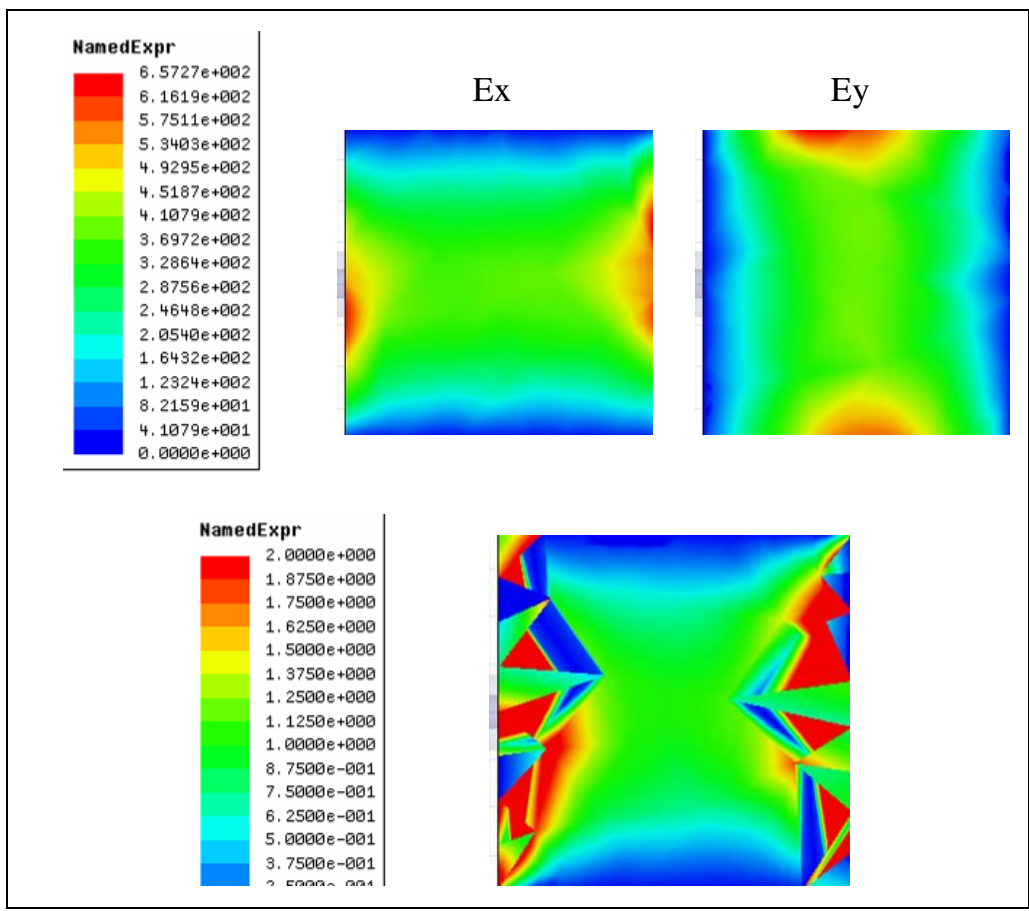

Figura 4-30. Relación axial diseño ajustado -Modo A 


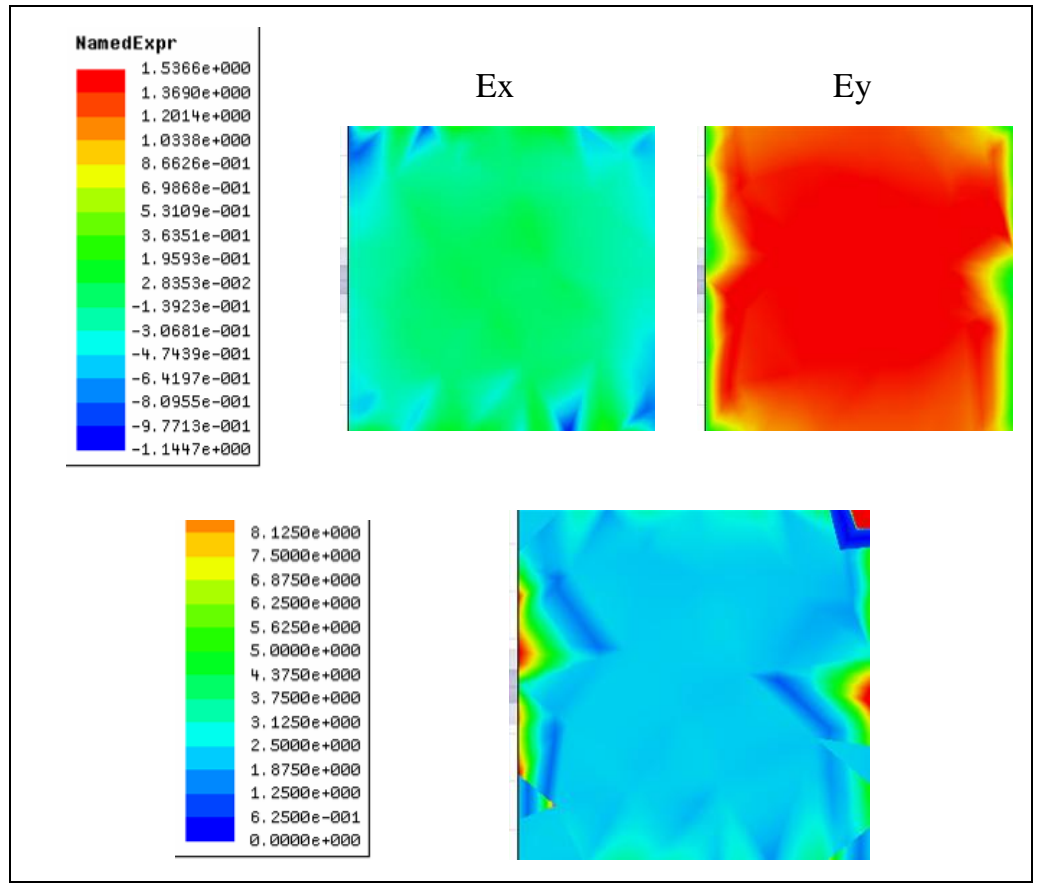

Figura 4-31. Desfase diseño ajustado -Modo A

Las simulaciones para el modo de funcionamiento $\mathrm{B}$, no son necesarias ya que anteriormente se demostró que los resultados en adaptación y polarización para el modo A se pueden extrapolar al modo B.

En conclusión, al introducir la transición el campo eléctrico en el conector no representa peligro para el aplicador. La mejora en adaptación es muy buena cuando se reduce la distancia de la alimentación al corto de 32,12 a 20,68 $\mathrm{mm}$ y las magnitudes son similares a las obtenidas sin transición. Por otro lado, la diferencia en adaptación y polarización cambiando la distancia entre el final del septum y el plano de apertura de aplicador de 106 a $97 \mathrm{~mm}$ no es muy significativa, aunque mejora un poco el campo de acción de la polarización circular en el plano de apertura.

\subsection{CONFRONTACIÓN}

Debido a la gran variedad de elementos a tratar es importante que la cobertura mínima del aplicador se amplíe con el fin de poder disminuir costos en un futuro. De esta manera, se aumenta el tamaño de la boca de radiación mediante un ensanchamiento tipo RIDGE de tres escalones, cuyas dimensiones son $5 \mathrm{~mm}$, teniendo una superficie de radiación de 105×105 mm (Figura 4-32). 


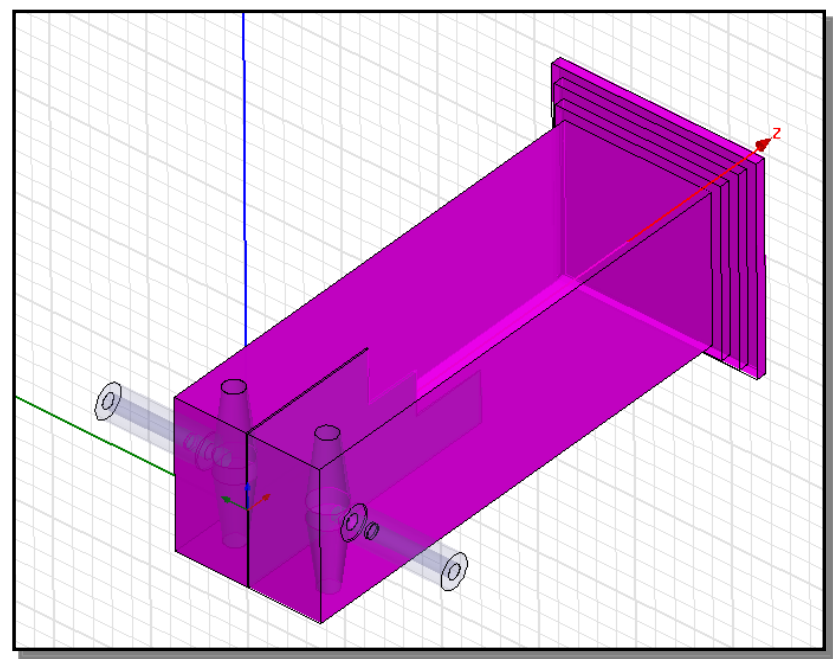

Figura 4-32. Ampliación Ridge de la cobertura

En las Figura 4-33 se muestra la magnitud de campo eléctrico y el desfase en el plano de apertura para los eje X y Y, se puede observar que el patrón sigue siendo el mismo de los apartados anteriores.

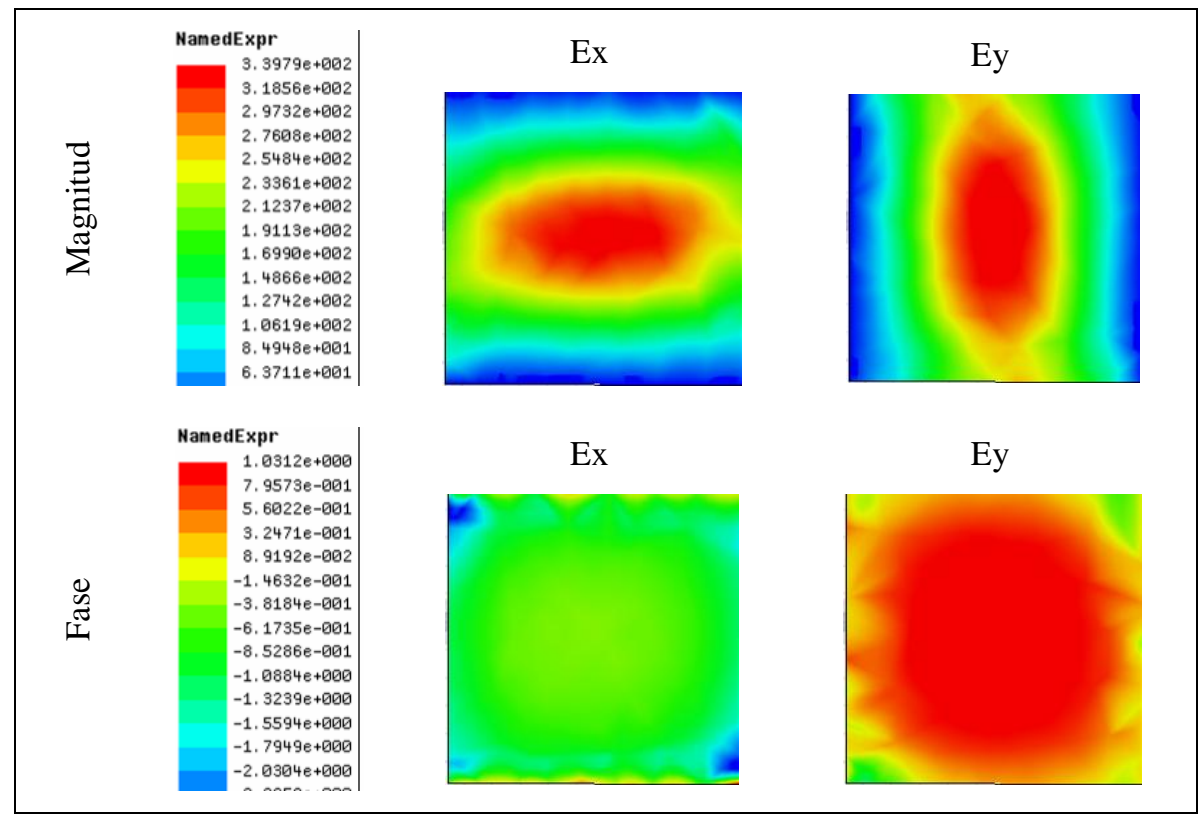

Figura 4-33. Campo eléctrico ampliación Ridge

En la Tabla 4-1 se muestra una comparación entre el aplicador sin ampliación y el aplicador con ampliación. Los parámetros $S_{11}$ y $S_{22}$ empeoran cerca de 1,2 dB estos valores se encuentran entre los encontrados en las diferentes simulaciones, por el contrario, el parámetro $S_{21}$ mejora a $-16,46 \mathrm{~dB}$ superando los valores alcanzados en 
las precedentes simulaciones. En relación con la polarización, esta se sigue manteniendo y la homogeneidad mejora en el plano de radiación.

Los resultados obtenidos sin variar las características encontradas en la parte de optimización y ampliando la apertura se aproximan mucho a los resultados originales. Sin embargo, es importante verificar que las distancias encontradas en la optimización sigan proporcionando el funcionamiento deseado con esta nueva configuración.

Tabla 4-1. Comparación ampliación con y sin Ridge

\begin{tabular}{|c|c|c|}
\hline & $\begin{array}{c}\text { APLICADOR SIN } \\
\text { RIDGE }\end{array}$ & APLICADOR CON RIDGE \\
\hline Cobertura & $75 \times 75 \mathrm{~mm}$ & $105 \times 105 \mathrm{~mm}$ \\
\hline $\mathrm{S}_{11}$ & $-21,24 \mathrm{~dB}$ & $-19,98 \mathrm{~dB}$ \\
\hline $\mathrm{S}_{22}$ & $-20,9 \mathrm{~dB}$ & $-19,74 \mathrm{~dB}$ \\
\hline $\mathrm{S}_{21} / \mathrm{S}_{12}$ & $-13,16 \mathrm{~dB}$ & $-16,46 \mathrm{~dB}$ \\
\hline 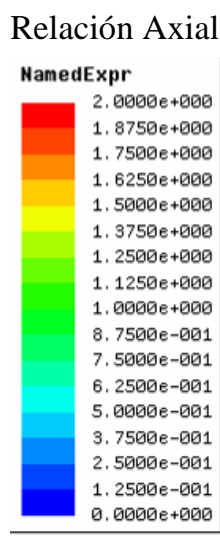 & & 1 \\
\hline 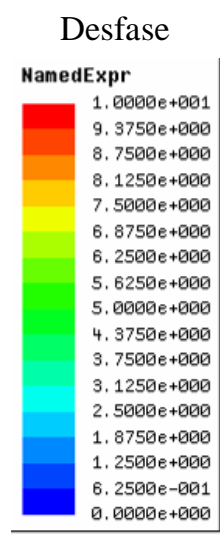 & 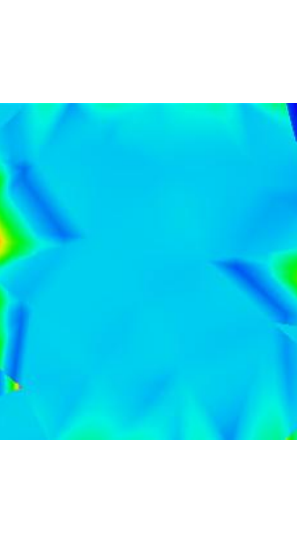 & 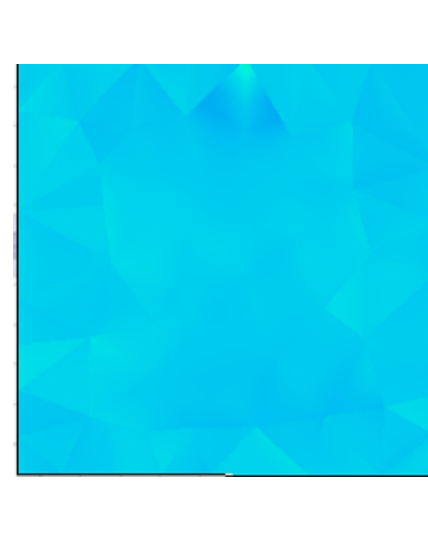 \\
\hline
\end{tabular}


Debido a que pico de la parametrización está en 20,48 mm (muy cerca de 20,68 mm anterior), no es necesario hacer una optimización o cambiar la distancia a corto (Figura 4-34).

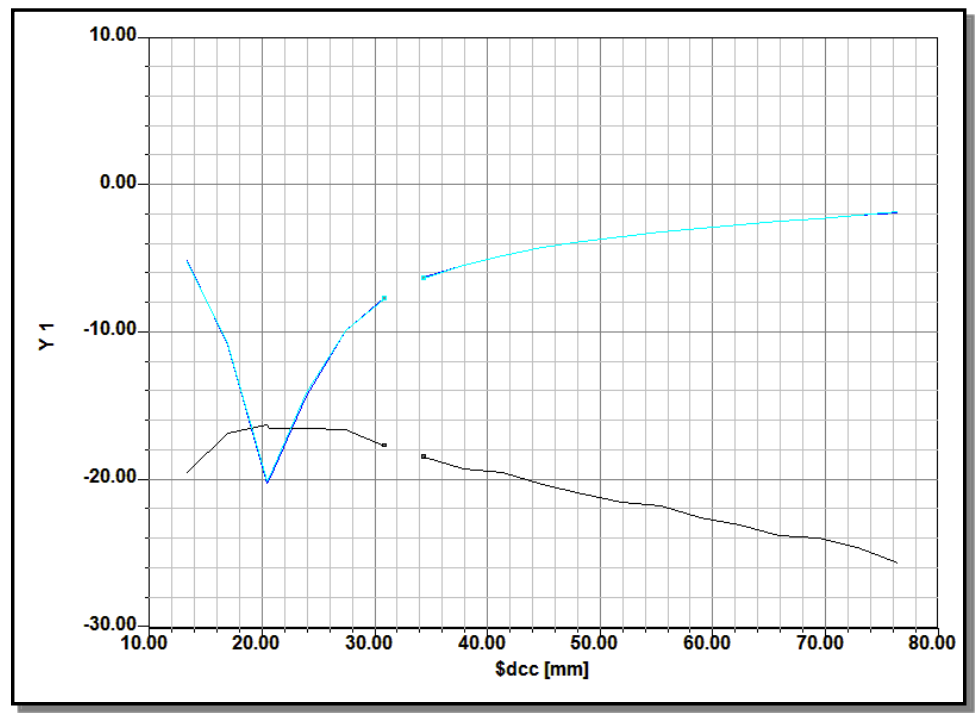

Figura 4-34. Parametrización alimentación ampliación Ridge

Adicionalmente, variando la longitud del aplicador se ve en las animaciones 5 y 6 que la relación axial es igual para todos los puntos mientras que el desfase solo es diferente de 2,5 $\pi$ para las distancias desde el septum de 87, 92, 122, 142 y $157 \mathrm{~mm}$, por lo tanto la distancia de $97 \mathrm{~mm}$ que se tenía anteriormente sigue siendo válida.

La ampliación mediante Ridge presenta algunos problemas para el equipo que construye el aplicador, por lo que se cambia esta ampliación por una bocina que se sobrepone a los escalones por la parte interior de los mismos (Figura 4-35).

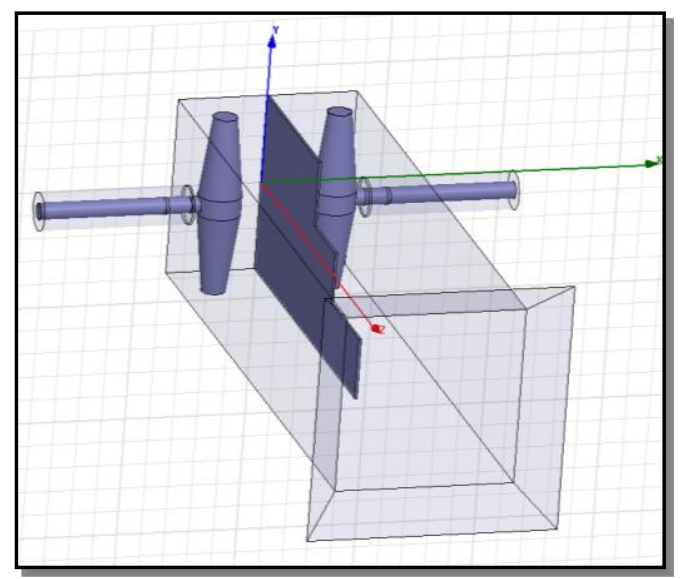

Figura 4-35. Ampliación bocina de la cobertura 
En la Figura 4-36 se observa que el patrón de campo eléctrico sigue siendo el mismo aunque las magnitudes de campo y las fases han variado con respecto al diseño de ampliación con Ridge.

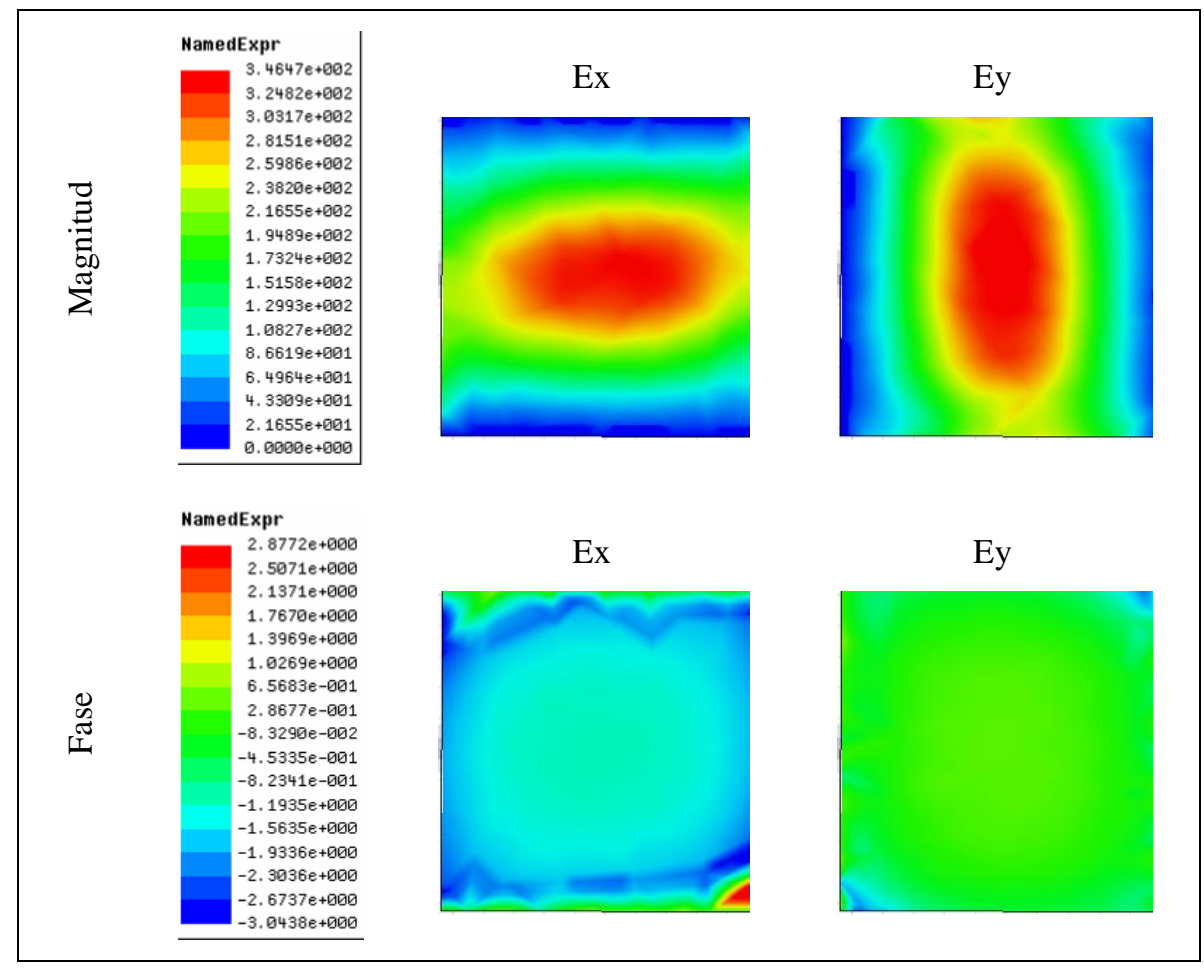

Figura 4-36.Campo eléctrico ampliación Bocina

En la Tabla 4-2 se hace una comparación entre la ampliación con Ridge y con bocina, los resultados de los parámetros de scattering para la bocina son similares a los obtenidos con Ridge y más cercanos a los resultados óptimos de cada uno encontrados a través de las diferentes simulaciones de este capítulo. 
Tabla 4-2. Comparación ampliación con Ridge y con Bocina

\begin{tabular}{|c|c|c|}
\hline & APLICADOR RIDGE & APLICADOR BOCINA \\
\hline $\mathrm{S}_{11}$ & $-19,98 \mathrm{~dB}$ & $-20,67 \mathrm{~dB}$ \\
\hline $\mathrm{S}_{22}$ & $-19,74 \mathrm{~dB}$ & $-21,23 \mathrm{~dB}$ \\
\hline $\mathrm{S}_{21} / \mathrm{S}_{12}$ & $-16,46 \mathrm{~dB}$ & $-15,8 \mathrm{~dB}$ \\
\hline 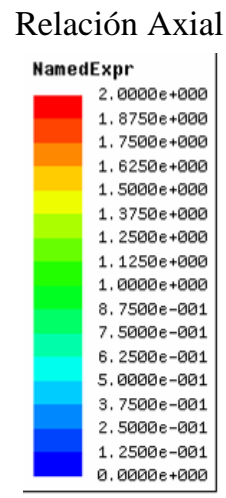 & & 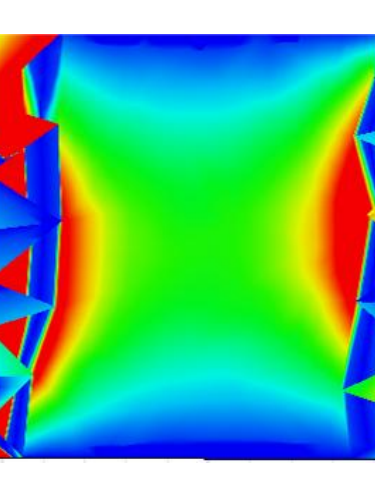 \\
\hline 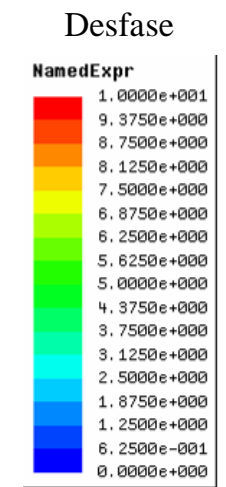 & & $\sqrt{-1}$ \\
\hline
\end{tabular}

Debido a que los resultados se acercan bastante entre sí, tanto en adaptación como en aislamiento y polarización, no se hacen más simulaciones. Así la distancia de la alimentación desde el corto es $20,68 \mathrm{~mm}$ y la longitud desde el septum hasta el plano de radiación es $97 \mathrm{~mm}$. El aplicador construido y la transición de microondas pueden verse en la Figura 4-37.

104 Tecnología de microondas en el sector de restauración de bienes culturales 

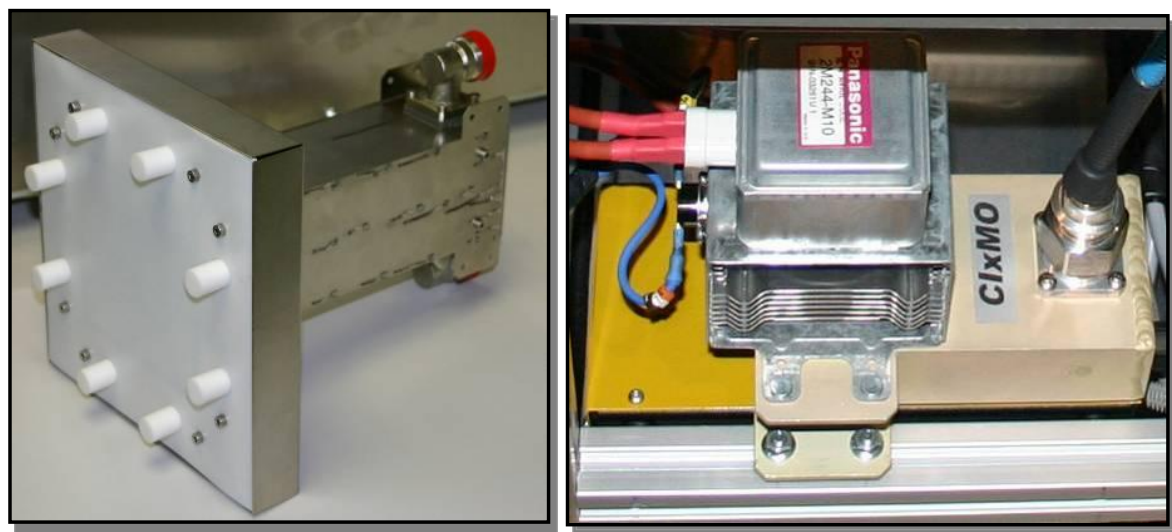

Figura 4-37. Aplicador y Magnetrón

\subsection{COMPROBACIÓN}

En este apartado se comprueba la adaptación del aplicador y su polarización, también se hacen pruebas para determinar zonas de seguridad alrededor del aplicador y la capacidad de atenuación del traje.

\subsubsection{Adaptación}

En la Tabla 4-3 se muestran las magnitudes medidas para los parámetros de adaptación de cada puerto y de aislamiento. Comparando estos resultados con los encontrados en las simulaciones, se mejora el parámetros de aislamiento en la frecuencia central, mientras que la adaptación se reduce en $6 \mathrm{~dB}$ manteniendo la diferencia entre un puerto y el otro en aproximadamente $0,5 \mathrm{~dB}$.

Tabla 4-3. Magnitud en dB de los parámetros $S$

\begin{tabular}{|c|c|c|c||}
\hline $\begin{array}{c}\text { FRECUENCIA } \\
\text { GHZ }\end{array}$ & $\begin{array}{c}\mathbf{S}_{\mathbf{1 1}} \\
\mathrm{dB}\end{array}$ & $\begin{array}{c}\mathbf{S}_{\mathbf{2 2}} \\
\mathrm{dB}\end{array}$ & $\begin{array}{c}\mathbf{S}_{\mathbf{2 1}} \\
\mathrm{dB}\end{array}$ \\
\hline 2,45 & $-14,5$ & $-13,84$ & $-26,72$ \\
\hline 2,4 & $-12,01$ & $-15,92$ & $-23,32$ \\
\hline 2,5 & $-16,2$ & $-11,46$ & $-19,53$ \\
\hline
\end{tabular}

\subsubsection{Polarización}

En las Tabla 4-4 y Tabla 4-5 puede verse que en ningún caso se tiene polarización circular a $2 \mathrm{~m}$ del plano de apertura, mientras a 6,5 y $14 \mathrm{~cm}$ sí se tiene una polarización circular. Por lo tanto los tratamientos tendrán que hacerse a distancias cortas desde el plano de apertura, como se ha planteado hasta el momento.

Por otro lado, tanto en el caso de maderas tangenciales como radiales, la polarización obtenida no se ve afectada después de atravesar una muestra de grosor 
menor a 2,5 cm. En cuanto a grosores mayores (como es el caso de la viga de $10 \mathrm{~cm}$ de grosor) se comprueba que la polarización se ve afectada, ya que las componentes de campo son atenuadas de diferente manera a lo largo de la muestra debido a su anisotropía.

Tabla 4-4. Polarización circular 1 alimentador

\begin{tabular}{|c|c|c|c|c|c|c|c|}
\hline \multirow{2}{*}{\multicolumn{2}{|c|}{1 Alimentador }} & \multicolumn{2}{|c|}{ Vertical } & \multicolumn{2}{|c|}{ Horizontal } & \multirow{3}{*}{$\begin{array}{c}\text { Rel. } \\
\text { Axial } \\
1,04\end{array}$} & \multirow{3}{*}{$\begin{array}{c}\text { Desfase } \\
\text { rad/pi }\end{array}$} \\
\hline & & \multirow{2}{*}{$\begin{array}{c}\text { Mag } \\
\text { S21 dB } \\
-34,1\end{array}$} & \multirow{2}{*}{$\begin{array}{c}\text { Fase } \\
\text { S21 }^{\circ} \\
280,98\end{array}$} & \multirow{2}{*}{$\begin{array}{c}\text { Mag } \\
21 \mathbf{d B} \\
-32,71\end{array}$} & \multirow{2}{*}{$\begin{array}{c}\text { fase } \\
\mathbf{S 2 1}^{\circ}\end{array}$} & & \\
\hline $2 \mathrm{~m}$ & Aire & & & & & & \\
\hline \multirow{3}{*}{$6,5 \mathrm{~cm}$} & Aire & $-9,95$ & 81,77 & $-9,23$ & 187,5 & 1,08 & 0,59 \\
\hline & $\begin{array}{c}\text { Madera } \\
\text { Tangencial }\end{array}$ & $-11,4$ & 45,37 & $-12,27$ & 143,6 & 0,93 & 0,55 \\
\hline & $\begin{array}{c}\text { Madera } \\
\text { Radial }\end{array}$ & $-11,5$ & 45,56 & $-12,38$ & 144 & 0,93 & 0,55 \\
\hline \multirow[b]{2}{*}{$14 \mathrm{~cm}$} & Aire & $-13,5$ & 234,81 & $-12,27$ & 312 & 1,10 & 0,43 \\
\hline & $\begin{array}{c}\text { Madera } \\
\text { Viga }\end{array}$ & $-17,7$ & 86,79 & $-18,99$ & 115,2 & 0,93 & 0,16 \\
\hline
\end{tabular}

Tabla 4-5. Polarización circular 2 alimentadores

\begin{tabular}{|c|c|c|c|c|c|c|c|}
\hline \multirow{2}{*}{\multicolumn{2}{|c|}{2 Alimentadores }} & \multicolumn{2}{|c|}{ Vertical } & \multicolumn{2}{|c|}{ Horizontal } & \multirow{3}{*}{$\begin{array}{c}\text { Rel. } \\
\text { Axial } \\
0,86\end{array}$} & \multirow{3}{*}{$\begin{array}{c}\begin{array}{c}\text { Desfase } \\
\text { rad/pi }\end{array} \\
0,03\end{array}$} \\
\hline & & \multirow{2}{*}{$\begin{array}{c}\text { Mag } \\
\text { S21 dB } \\
-32,2\end{array}$} & \multirow{2}{*}{$\begin{array}{l}\text { Fase } \\
\mathbf{S 2 1}^{\circ}\end{array}$} & \multirow{2}{*}{$\begin{array}{c}\text { Mag } \\
\text { S21 dB } \\
-37,49\end{array}$} & \multirow{2}{*}{$\begin{array}{l}\text { Fase } \\
\mathbf{S 2 1}^{\circ} \\
272,5\end{array}$} & & \\
\hline $2 \mathrm{~m}$ & Aire & & & & & & \\
\hline \multirow{3}{*}{$6,5 \mathrm{~cm}$} & Aire & $-8,56$ & 89,98 & $-14,96$ & 21,8 & 0,57 & 0,38 \\
\hline & $\begin{array}{c}\text { Madera } \\
\text { Tangencial }\end{array}$ & $-12,1$ & 37,63 & $-14,75$ & 341,6 & 0,82 & 1,69 \\
\hline & $\begin{array}{c}\text { Madera } \\
\text { Radial }\end{array}$ & $-12,3$ & 37,75 & $-14,65$ & 337,9 & 0,84 & 1,67 \\
\hline \multirow[b]{2}{*}{$14 \mathrm{~cm}$} & Aire & $-12,5$ & 245,57 & $-16,92$ & 194,8 & 0,74 & 0,28 \\
\hline & $\begin{array}{c}\text { Madera } \\
\text { Viga }\end{array}$ & $-19,6$ & 49,95 & $-21,9$ & 48,89 & 0,90 & 0,01 \\
\hline
\end{tabular}

En el caso de la Tabla 4-5 las condiciones se acercan a las de polarización circular. La diferencia entre los resultados del diseño y las medidas, se deben a que en el 
diseño los dos puertos están en fase mientras en la realidad los magnetrones son distintos, por lo tanto oscilan con fases distintas y aleatorias, lo que afecta las componentes de campo de manera diferente.

En resumen, los tratamientos deben aplicarse cerca al plano de apertura del aplicador y el grosor de las maderas a tratar no debe superar los $10 \mathrm{~cm}$. También se puede asegurar una polarización circular con un tratamiento de $1 \mathrm{~kW}$, mientras para un tratamiento de $2 \mathrm{~kW}$ ésta se consigue sólo de manera aproximada. En el Apéndice V. i se encuentran los parámetros de scattering.

\subsubsection{Medidas de nivel de Campo}

\subsubsection{Campo en la superficie irradiada}

Debido a que la alta directividad del aplicador puede poner en peligro la electrónica de la caja de control, estas pruebas se hacen con una muestra de madera de 20x20x5 cm que absorba parte de la energía en la dirección de máxima radiación.

Las medidas deben hacerse en campo lejano, es decir con el equipo de medida de radiación ubicado a más de $37,7 \mathrm{~cm}$ de la apertura del aplicador.

$$
\begin{gathered}
E_{\text {lejano }}=\frac{2 \times D^{2}}{\lambda}=\frac{2 \times(15,2 \mathrm{~cm})^{2}}{12,24 \mathrm{~cm}}=37,7 \mathrm{~cm} \\
D=\frac{\sqrt{(21,5 \mathrm{~cm})^{2}+(21,5 \mathrm{~cm})^{2}}}{2}=15,3 \mathrm{~cm}
\end{gathered}
$$

Como resultado se obtiene el campo producido alrededor del aplicador sobre planos paralelos a la superficie irradiada. El experimento establece el patrón de campo a 2,5 $\mathrm{m}$ desde el cetro del aplicador en diferentes alturas. La muestra está a $80 \mathrm{~cm}$ de altura sobre una mesa redonda con un radio de $75 \mathrm{~cm}$.

Debido a que el magnetrón tarda un tiempo en estabilizarse, antes de representar los datos es necesario retirar de la población las dos primeras medidas de cada punto. La Figura 4-38 muestra la mediana de campo obtenida para una potencia de $1 \mathrm{~kW}$ y la Figura 4-39 para una potencia de $2 \mathrm{~kW}$ (Datos medidos en el Apéndice V. ii). Ambas figuras tienen gráficos polares, en los que la intensidad de campo en $\mathrm{V} / \mathrm{m}$ esta en el eje y el círculo verde identifica el límite de exposición en áreas públicas a $2,45 \mathrm{GHz}(61,4 \mathrm{~V} / \mathrm{m})[36,46]$.

En la Figura 4-38 se observa que los máximos son mayores que las medianas en reducidos casos, por ejemplo a una altura de $1,75 \mathrm{~m}$ en la dirección $0^{\circ}$, donde la intensidad de campo es 46,82 V/m. Este valor de máximo se tiene en cuenta como el caso más pesimista a pesar de durar tan sólo un segundo; además se verifica que el valor del máximo corresponde sólo a la primera medida del conjunto tenido en cuenta y el resto de medidas se acercan a la mediana (índice de dispersión Apéndice V. ii), dando mucha más fiabilidad sobre las zonas de seguridad a establecer.

En el caso de un tratamiento de $2 \mathrm{~kW}$ (Figura 4-39), los máximos y las medianas son similares en los cuatro casos. El caso peor se encuentra a $1 \mathrm{~m}$ de altura en la 
dirección $210^{\circ}$, donde la intensidad de campo es $86,21 \mathrm{~V} / \mathrm{m}$ a 2,5 $\mathrm{m}$ del centro del aplicador.

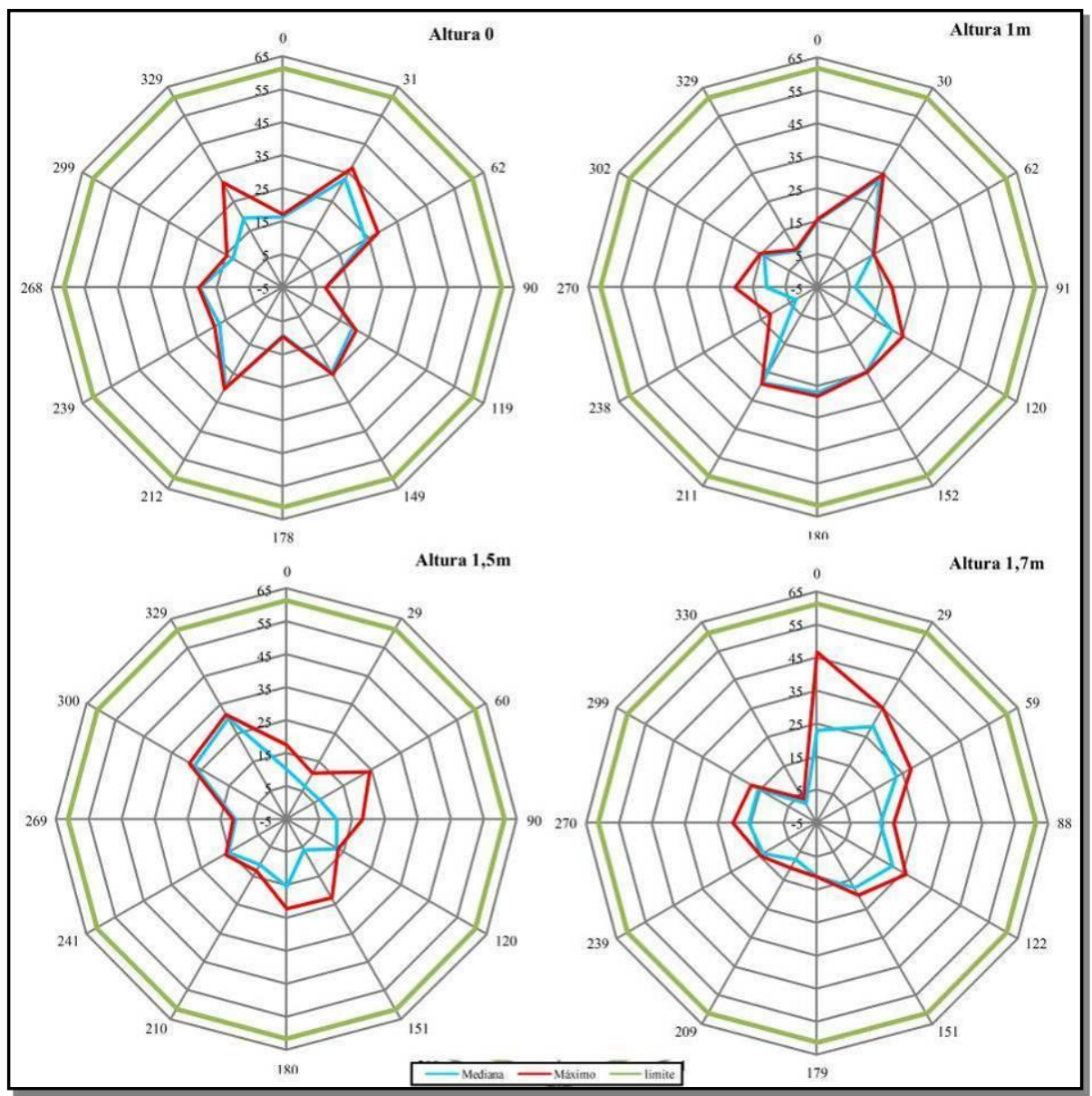

Figura 4-38.Intensidad de Campo Eléctrico en la superficie irradiada -1 kW 


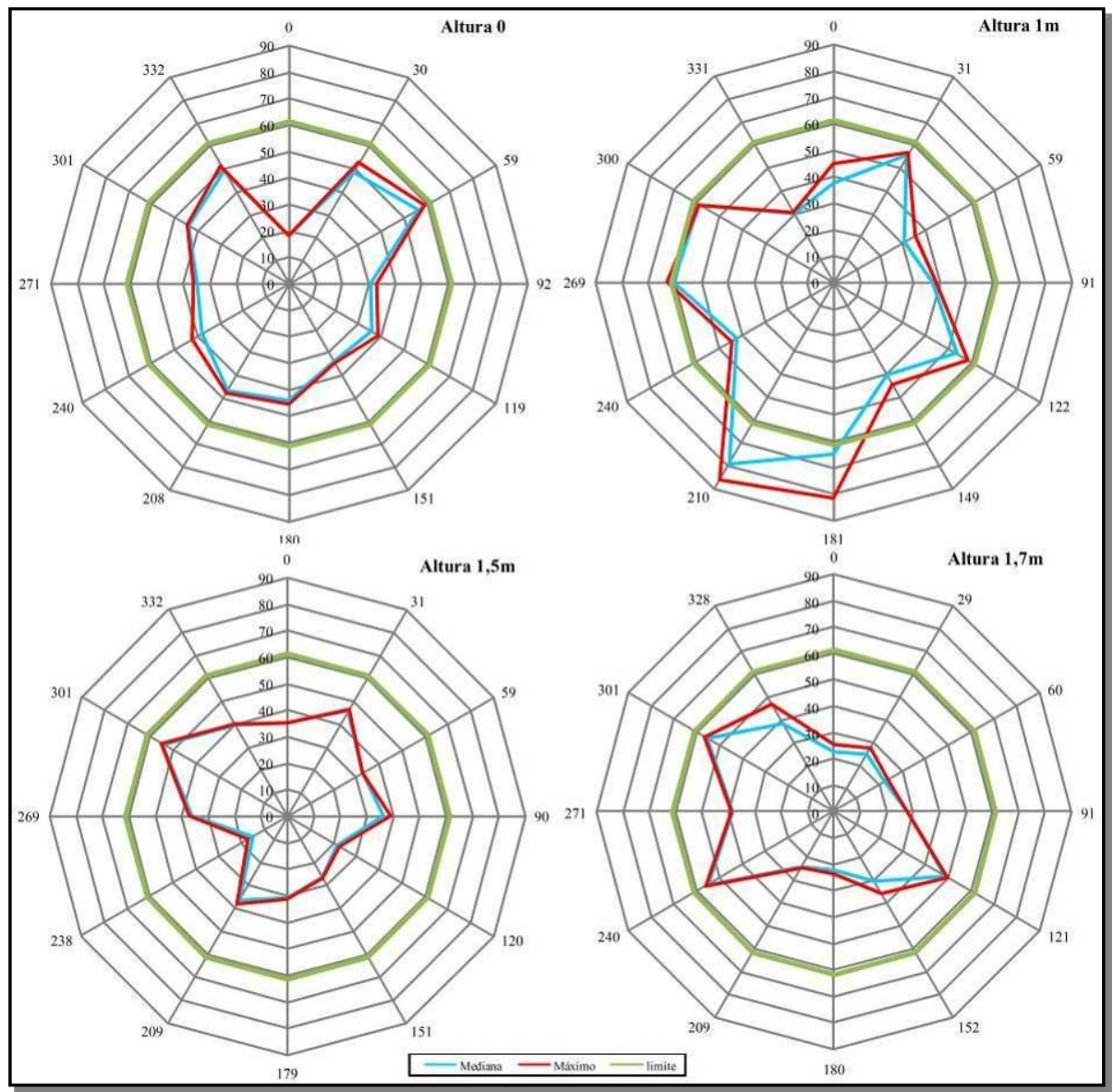

Figura 4-39. Campo Eléctrico en la superficie irradiada- $2 \mathrm{~kW}$

Con los datos anteriores se pueden establecer las zonas de seguridad. Sabiendo que en campo lejano:

$$
\frac{\left|E_{1}\right|^{2}}{\left|E_{2}\right|^{2}}=\frac{r_{2}^{2}}{r_{1}^{2}}
$$

y conociendo la intensidad de campo a una distancia del aplicador y el límite establecido por la normativa, se pueden calcular los radios de seguridad como:

$$
R=\sqrt{\frac{(\text { Distancia } m)^{2} \times(\text { Máximo } V / m)^{2}}{(\text { Límite } V / m)^{2}}}
$$

de esta manera se calculan dos radios de seguridad:

R1 se calcula a partir del límite de área pública $61,4 \mathrm{~V} / \mathrm{m}$. 
R2 se calcula a partir del límite de áreas de trabajo de 137 V/m [46].

El exterior del R1 se establece como área pública, el área comprendida entre el R1 y R2 como zona de trabajo donde el operario puede circular y el interior de R2 se establece como área restringida donde no se puede circular durante un tratamiento. En la Tabla 4-6 se muestran los radios de seguridad y en la Figura 4-40 se representan las zonas de seguridad alrededor del aplicador.

Tabla 4-6.Radios de seguridad- superficie irradiada

\begin{tabular}{||c|c|c|c||}
\hline RADIO & Limite & $\mathbf{1 k W}$ & $\mathbf{2 k W}$ \\
\cline { 3 - 4 } $\mathbf{2 , 5 m}$ & $\mathbf{V} / \mathbf{m}$ & $\begin{array}{c}\mathbf{4 6 , 8 2} \\
\mathbf{V} / \mathbf{m}\end{array}$ & $\begin{array}{c}\mathbf{8 6 , 2 1} \\
\mathbf{V} / \mathbf{m}\end{array}$ \\
\hline $\mathbf{R 1}(\mathbf{m})$ & 61,4 & 1,90 & 3,51 \\
\hline R2 (m) & 137 & 0,85 & 1,57 \\
\hline
\end{tabular}

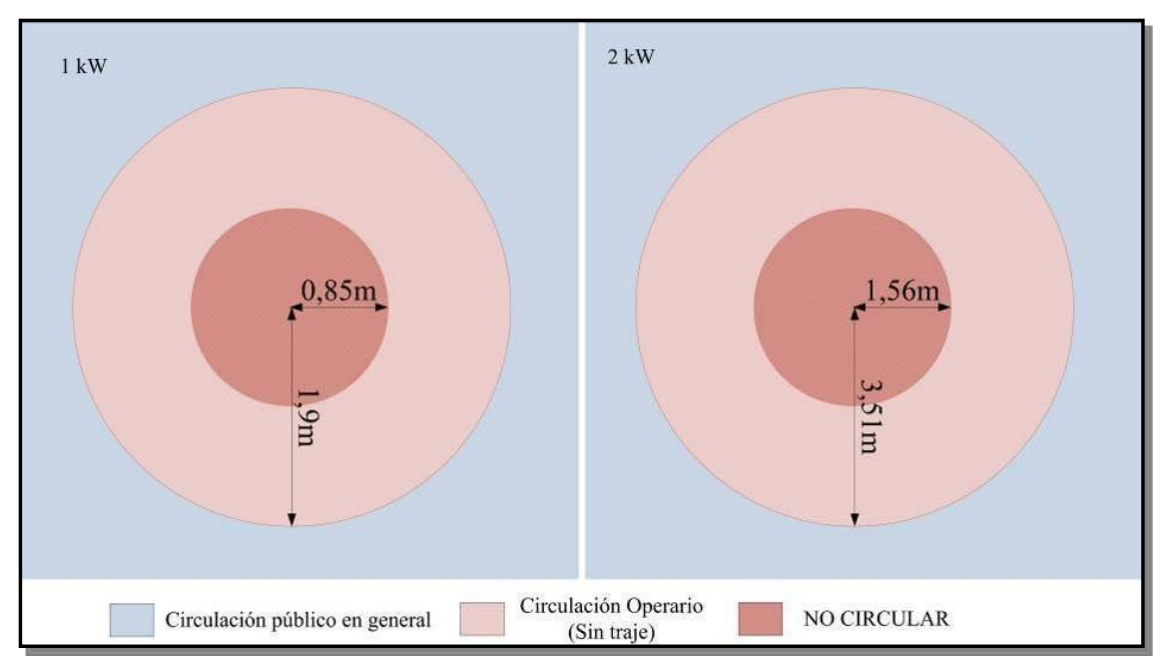

Figura 4-40. Zonas de seguridad - superficie irradiada

La pieza tratada para este experimento tiene una superficie irradiada de $20 \times 20 \mathrm{~cm}$, dimensiones similares a las de la apertura del aplicador, por lo que se puede sugerir que para el tratamiento de piezas de mayor superficie los radios establecidos deben marcarse cuando se tratan las esquinas de la piezas, ya que en los puntos interiores las microondas son absorbidas en su mayor parte por la pieza de tratada.

\subsubsection{Campo en el corte principal del diagrama de radiación}

Con el objetivo de considerar el peor de los casos estas pruebas se hacen sin muestra a tratar y con un tratamiento de $2 \mathrm{~kW}$ durante 10 segundos, se establece el patrón de campo a $2 \mathrm{~m}$ desde el plano de apertura del aplicador (Figura 4-41). 


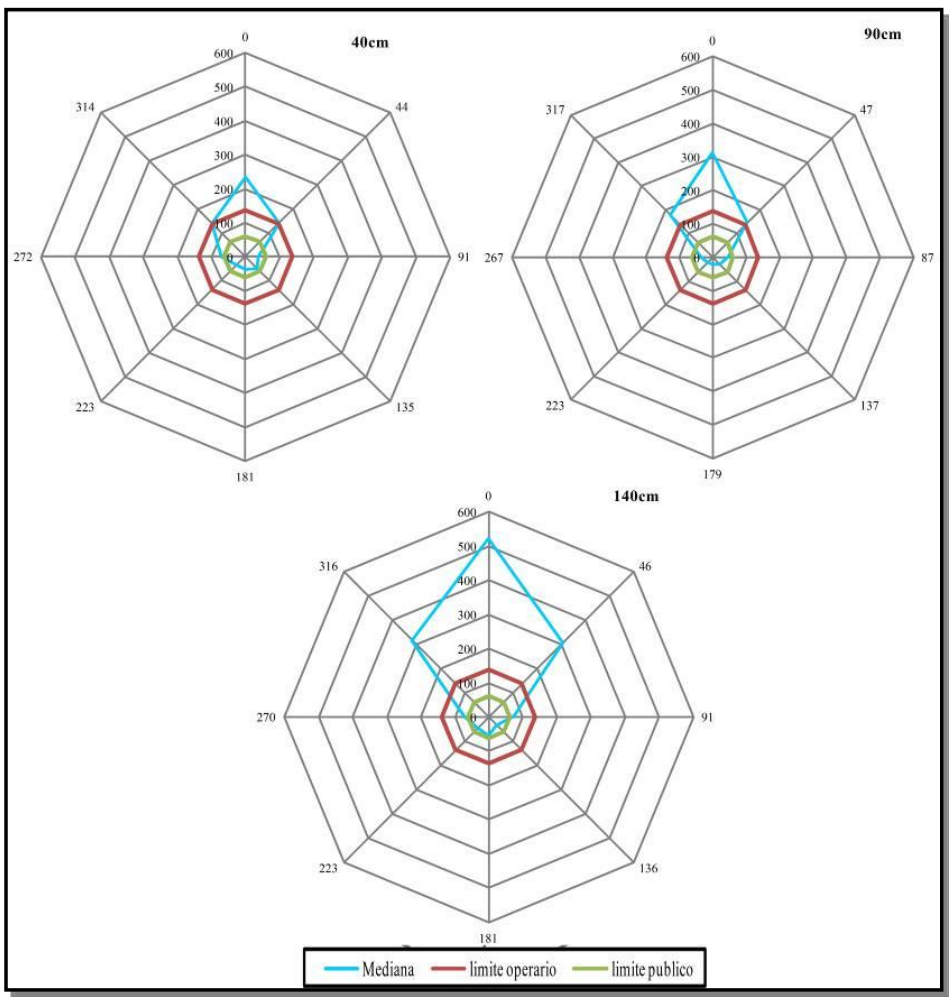

Figura 4-41. Campo corte principal.

Aunque la máxima intensidad de campo debería obtenerse en la línea de vista con el aplicador $(90 \mathrm{~cm})$, ésta se encuentra a la altura de $140 \mathrm{~cm}$; esto puede deberse a las reflexiones sobre la mesa en la que está colocado el aplicador.

Con los resultados, se puede afirmar que detrás del aplicador (ángulos entre $90^{\circ} \mathrm{y}$ $270^{\circ}$ ) no se sobrepasan los límites para zonas de trabajo o áreas públicas más allá de una distancia de $2 \mathrm{~m}$. Con el fin de establecer estos radios de manera más exacta se toma la máxima intensidad encontrada en dichos ángulos $(53,09 \mathrm{~V} / \mathrm{m})$, sabiendo que esta intensidad corresponde a una distancia de $2 \mathrm{~m}$ desde el plano de apertura y conociendo los límites de la normativa, se tiene que:

$$
\begin{aligned}
& R 1=\sqrt{\frac{(2 \mathrm{~m})^{2} \times(53,09 \mathrm{~V} / \mathrm{m})^{2}}{(61,4 \mathrm{~V} / \mathrm{m})^{2}}}=1,73 \mathrm{~m} \\
& R 2=\sqrt{\frac{(2 \mathrm{~m})^{2} \times(53,09 \mathrm{~V} / \mathrm{m})^{2}}{(137 \mathrm{~V} / \mathrm{m})^{2}}}=0,77 \mathrm{~m}
\end{aligned}
$$

Como es lógico, la máxima intensidad de campo se encuentra en la dirección de máxima radiación $\left(0^{\circ}\right)$. En los tres gráficos puede verse que el límite de trabajo es 
superado para los ángulos comprendidos entre $315^{\circ}$ y $45^{\circ}$ (la máxima intensidad en estos ángulos es 520,13 V/m). En el caso del límite de áreas públicas el rango de ángulos está comprendido entre $285^{\circ}$ y $75^{\circ}$, siguiendo la formulación y aprovechando la simetría del lóbulo de radiación, se muestran los radios para los diferentes rangos de ángulos en la Tabla 4-7 y Tabla 4-8.

Tabla 4-8. R2 en el corte principal

Tabla 4-7. R1 en el corte principal

\begin{tabular}{||c|c|c||}
\hline Ángulos & $\begin{array}{c}\text { MAX } \\
(\mathbf{V} / \mathbf{m})\end{array}$ & $\begin{array}{c}\text { R1 } \\
(\mathbf{m})\end{array}$ \\
\hline$[285-75]$ & 520,13 & 16,94 \\
\hline $\begin{array}{c}{[75-90]} \\
{[270-285]}\end{array}$ & 100 & 3,26 \\
\hline$[90-270]$ & 53,09 & 1,73 \\
\hline
\end{tabular}

\begin{tabular}{|c|c|c||}
\hline Ángulos & $\begin{array}{c}\text { MAX } \\
(\mathbf{V} / \mathbf{m})\end{array}$ & $\begin{array}{c}\text { R2 } \\
(\mathbf{m})\end{array}$ \\
\hline$[315-45]$ & 520,13 & 7,59 \\
\hline $\begin{array}{c}{[45-70]} \\
{[290-315]}\end{array}$ & 316,85 & 4,62 \\
\hline $\begin{array}{c}{[70-90]} \\
{[270-209]}\end{array}$ & 137 & 2 \\
\hline$[90-270]$ & 53,09 & 0,77 \\
\hline
\end{tabular}

De la misma manera que en el apartado 4.5.3.1 la zona exterior al radio R1 se considera área pública y la zona de trabajo es el área comprendida entre R1 y R2, en la cual el operario puede circular sin traje apantallado.

Para completar el estudio es necesario definir dos zonas más de seguridad que vienen dadas por el radio R3, que tiene en cuenta el límite en áreas de trabajo que es $137 \mathrm{~V} / \mathrm{m}$. La primera zona es en la que el operario debe vestir obligatoriamente el traje y otra es en la que bajo ninguna circunstancia deberá circular con un tratamiento en marcha. Se hacen las mismas medidas pero con el traje apantallado (Figura 4-42), los resultados se muestran en la Figura 4-43 (Apéndice V. iii).
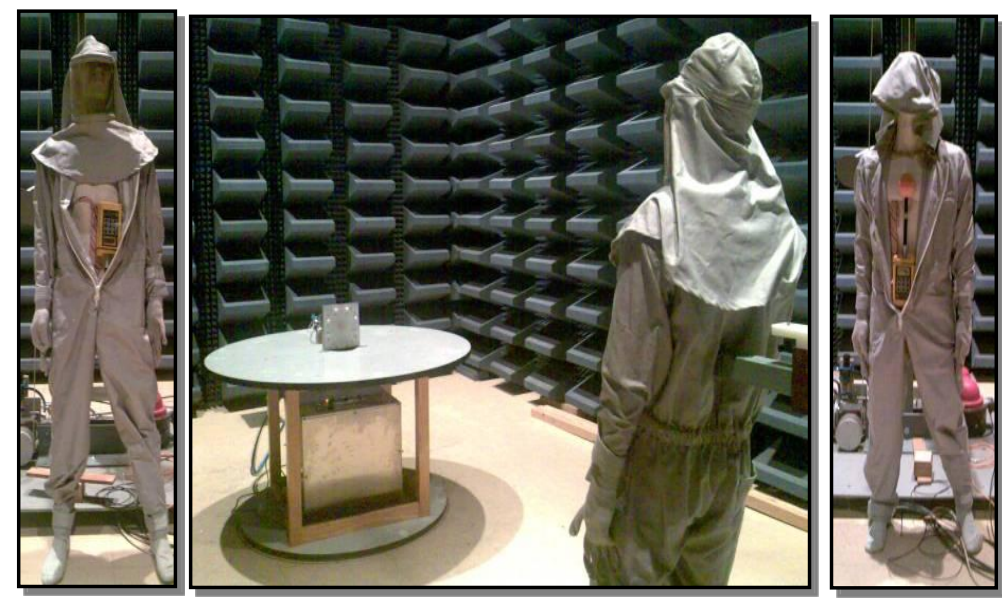

Figura 4-42. Medidas con el traje apantallado.

112 Tecnología de microondas en el sector de restauración de bienes culturales 


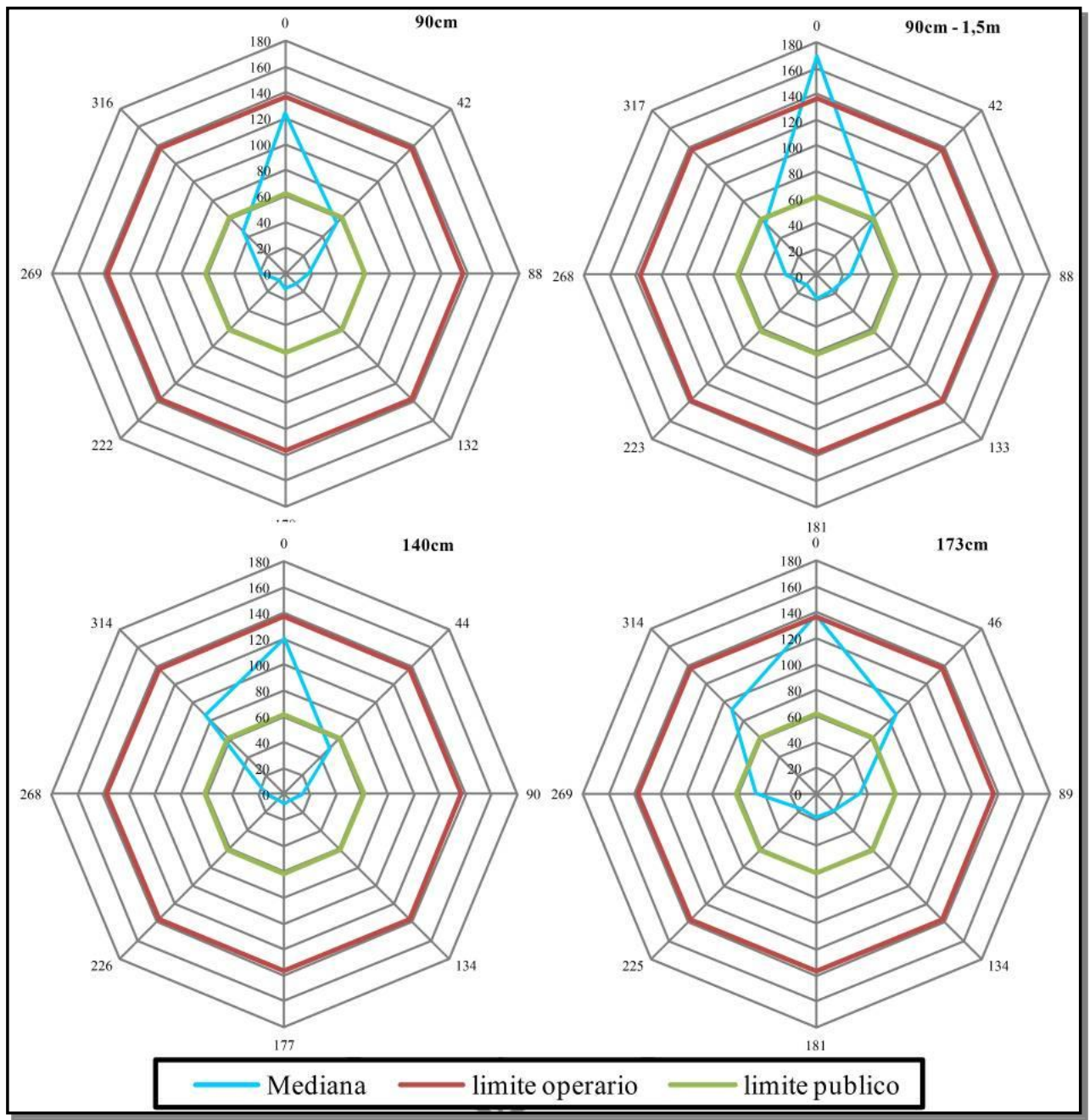

Figura 4-43. Campo corte principal- con traje.

El radio R3 se calcula de la misma manera como se ha calculado el radio R2, pero los datos de intensidad de campo son los de las medidas con el traje. El peor de los tres casos a $2 \mathrm{~m}$ del aplicador ${ }^{10}$ se encuentra a $1,73 \mathrm{~m}$ de altura ${ }^{11}$, basándose en los rangos de ángulos establecidos para el radio R2 y su máxima intensidad de campo se calcula el R3 que se muestra en la Tabla 4-9.

${ }^{10}$ Figura 4-43 menos el grafico superior derecho, titulado $90 \mathrm{~cm}-1,5 \mathrm{~m}$. En la página siguiente se justifica el porqué de esta diferencia.

${ }^{11}$ Corresponde a la altura de la cabeza, donde el traje tiene rejilla en lugar de tela. 
En el interior del radio R3 el operario no podrá circular bajo ninguna circunstancia y en el área comprendida entre R2 y R3 el operario deberá vestir obligatoriamente el traje apantallado si desea circular.

Tabla 4-9. R3 en el corte principal

\begin{tabular}{||l|c|c||}
\hline \multicolumn{1}{|c|}{ Ángulos } & MAX (V/m) & R3 (m) \\
\hline$[315-45]$ & 137 & 2 \\
\hline$[45-70][290-315]$ & 97,36 & 1,42 \\
\hline$[70-90][270-209]$ & 60 & 0,87 \\
\hline$[90-270]$ & 45,97 & 0,67 \\
\hline
\end{tabular}

En la Figura 4-44 se muestra el diagrama de las zonas de seguridad para un tratamiento de $2 \mathrm{~kW}$. Hay que tener en cuenta que el valor exacto de los distintos radios depende del tipo y grosor de la pieza a tratar, y por supuesto del tratamiento aplicado. Ahora bien, los radios de la Tabla 4-7, Tabla 4-8 y Tabla 4-9 se han estimado para el peor de los casos, por lo que se consideran validos para cualquier tipo de tratamiento.

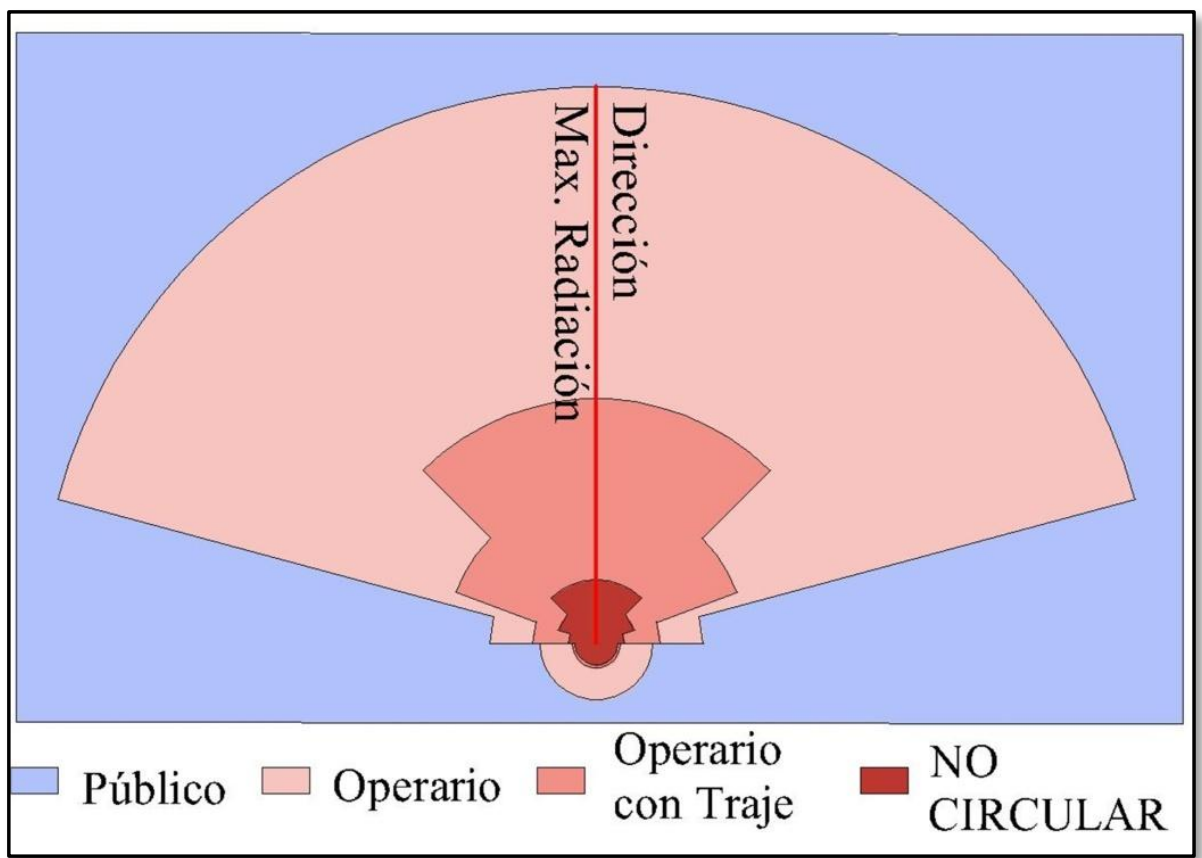

Figura 4-44. Zonas de seguridad - corte principal

En los gráficos superiores de la Figura 4-43 se tiene la intensidad de campo a una altura de las caderas $(90 \mathrm{~cm})$ dentro del traje apantallado a dos distancias del plano

114 Tecnología de microondas en el sector de restauración de bienes culturales 
de apertura del aplicador: 1,5 y $2 \mathrm{~m}$. Estos valores se han medido para estimar el error relativo de la formula en el caso del traje apantallado:

$$
\begin{gathered}
\frac{\left|E_{1}\right|^{2}}{\left|E_{2}\right|^{2}}=\frac{r_{2}{ }^{2}}{r_{1}{ }^{2}} \Rightarrow \frac{(124,1 \mathrm{~V} / \mathrm{m})^{2}}{(170,25 \mathrm{~V} / \mathrm{m})^{2}}=\frac{(1,5 \mathrm{~m})^{2}}{(2 \mathrm{~m})^{2}} \Rightarrow 0,5313=0,5625 \\
\text { Error Relativo }=\frac{|0,5313-0,5625|}{0,56525} \times 100=5,5 \%
\end{gathered}
$$

El error de 5,5\% en la dirección de máxima radiación (ángulos entre $315^{\circ}$ y $45^{\circ}$ ) significa aumentar R3 un máximo de $11 \mathrm{~cm}$ y en relación con la intensidad de campo representa $7,53 \mathrm{~V} / \mathrm{m}$. Sin embargo se recomienda al operario no circular frente al aplicador durante un tratamiento aunque se establezcan los radios de seguridad y vista el traje.

Volviendo al caso del apartado 4.5.3.1 (dirección de máxima radiación al piso), no se repiten las medidas, sino que el radio R3 se obtiene a partir de la atenuación que presenta el traje. En la Tabla 4-10 se calcula la atenuación que presenta el traje a diferentes alturas y distancias en la dirección de máxima radiación.

Tabla 4-10. Atenuación del traje

\begin{tabular}{||c|c|c|c||}
\hline $\begin{array}{c}\text { Altura } \\
(\mathbf{c m})\end{array}$ & $\begin{array}{c}|\mathbf{E}| \text { Sin traje } \\
(\mathbf{V} / \mathbf{m})\end{array}$ & $\begin{array}{c}|\mathbf{E}| \text { Con traje } \\
(\mathbf{V} / \mathbf{m})\end{array}$ & $\begin{array}{c}\text { Atenuación } \\
(\mathbf{d B})\end{array}$ \\
\hline 140 a $2 \mathrm{~m}$ & 520,13 & 120,22 & 12,72 \\
\hline 90 a 2 m & 313,33 & 124,1 & 8,04 \\
\hline 90 a $1,5 \mathrm{~m}$ & $417,76 *$ & 170,25 & 7,79 \\
\hline
\end{tabular}

*Calculada

La menor de las atenuaciones presentadas por el traje está a la altura de $90 \mathrm{~cm}$, teniendo en cuenta el error de 5,5\% el peor caso será 7,4 dB. En base a esto, teniendo en cuenta que la máxima intensidad de campo es $86,21 \mathrm{~V} / \mathrm{m}$ a $2,5 \mathrm{~m}$ para el tratamiento de $2 \mathrm{~kW}^{12}$ y modificando el límite de la formula al multiplicarlo por la atenuación en lineal $(2,34)$, se calcula el radio $\mathrm{R} 3$ como:

$$
R 3=\sqrt{\frac{(2,5 \mathrm{~m})^{2} \times(86,21 \mathrm{~V} / \mathrm{m})^{2}}{(137 \mathrm{~V} / \mathrm{m})^{2}}}=0,67 \mathrm{~m}
$$

La Figura 4-45 muestras las zonas de seguridad completas en el caso del apartado 4.5.3.1 para un tratamiento de $2 \mathrm{~kW}$.

${ }^{12}$ Para conocer el radio para un tratamiento de $1 \mathrm{~kW}$ se debe dividir por 2. 


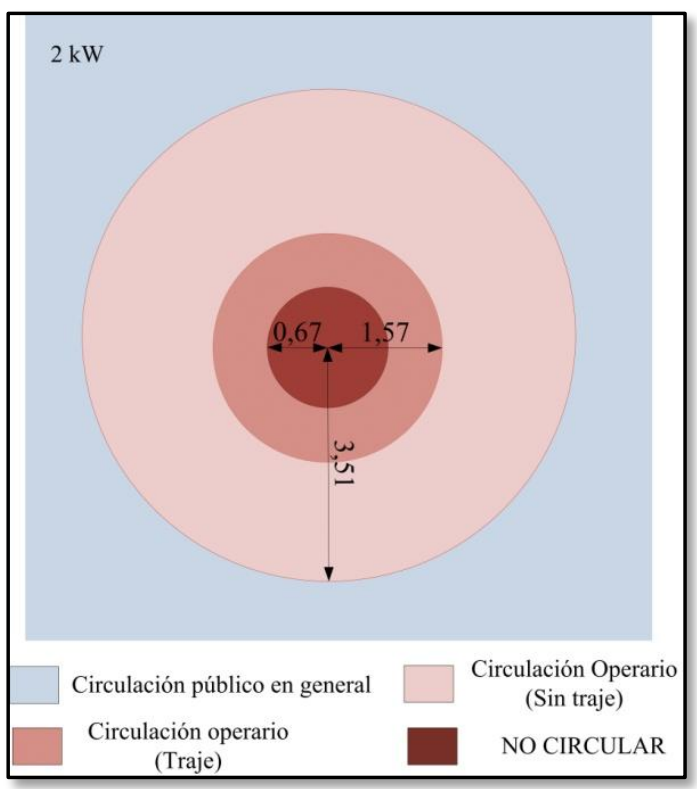

Figura 4-45. Zona de seguridad completas en superficie irradiada

\subsection{MEDIDAS DE TEMPERATURA}

\subsubsection{Temperatura en aplicación central}

Las muestras tangenciales de madera joven de 20x20x2,5 cm están compuestas por dos tablas pegadas, para evitar que esto afecte los resultados al radiar sobre la juntura el centro de la radiación y los puntos de control de temperatura se mueven hacia el lado más amplio como se muestra en la Figura 4-46.

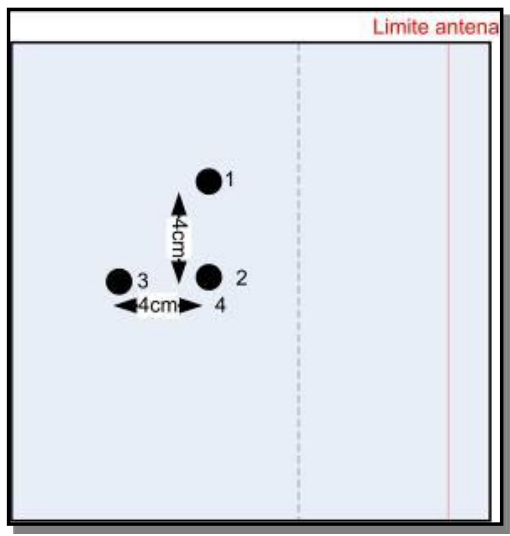

Figura 4-46. Ubicación de sondas muestras tangenciales jóvenes

Se utiliza una cámara de video para verificar el correcto funcionamiento de los magnetrones desde fuera de la cámara, el horno microondas se utiliza con el fin de

$\overline{116 \text { Tecnología de microondas en el sector de restauración de bienes culturales }}$ 
proteger el equipo eléctrico de las sondas ópticas de un posible fallo a causa de la radiación (Figura 4-47).
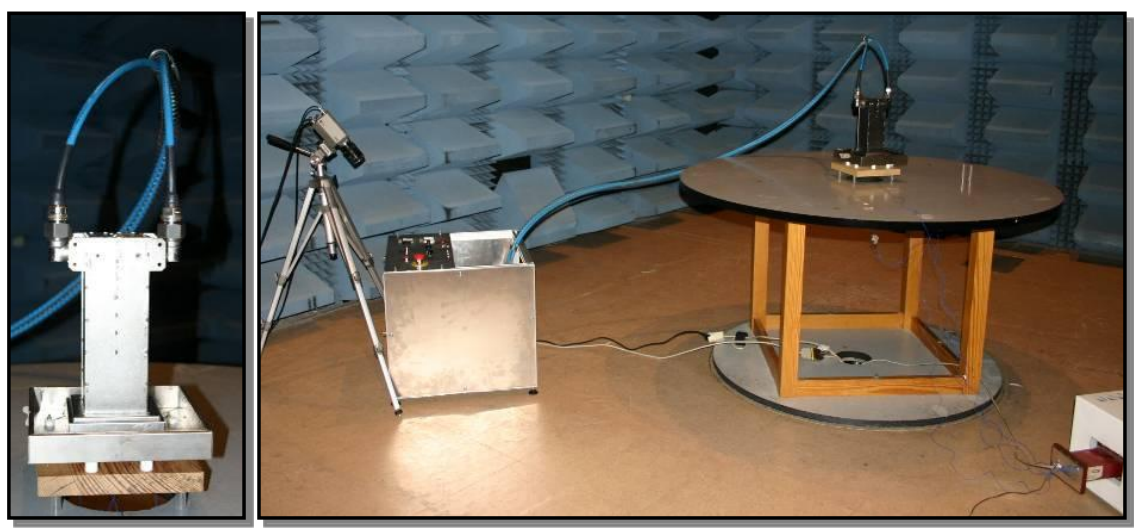

Figura 4-47. Medidas Temperatura aplicación central

Los valores de la mediana de temperatura correspondientes a las cinco muestras medidas se representan en la Figura 4-48. En el caso de las muestras radiales sólo son validas tres, porque el movimiento del centro de la aplicación para una de las muestras genera un mayor calentamiento en el punto paralelo debido a la cercanía con el límite de la muestra y otra fue radiada accidentalmente antes de tomar los datos de temperatura por lo que su temperatura inicial es superior a la de las demás.

Se puede afirmar al igual que en el CAPÍTULO 3, que las tablas tangenciales se calientan más que las radiales, al igual que las muestras de madera joven más que las de antigua debido a su mayor contenido de agua. Por otro lado la alta temperatura alcanzada en las muestras jóvenes puede también deberse a la cercanía del centro de la aplicación con los límites de la tabla generando un mayor calentamiento en los tres puntos.

La tendencia de calentamiento en los puntos controlados es la esperada y es similar para todas las muestras medidas. En cuanto a la validez de los datos, el índice de dispersión está por debajo de 10\% (Apéndice VI) para los tres tipos de muestras, siendo menor y más uniforme que los datos obtenidos en las pruebas hechas con el aplicador lineal.

Finalmente, la temperatura máxima alcanzada en el interior de las maderas antiguas no supera los $40{ }^{\circ} \mathrm{C}$, valor muy por debajo del límite propuesto de $60{ }^{\circ} \mathrm{C}$. La temperatura alcanzada en los puntos periféricos ronda los $30^{\circ} \mathrm{C}$ y es inferior al valor máximo de temperatura en superficie $\left(35^{\circ} \mathrm{C}\right)$.

La diferencia de las muestras antiguas y las jóvenes es sólo una cuestión de magnitud, ya que el comportamiento es el mismo. 


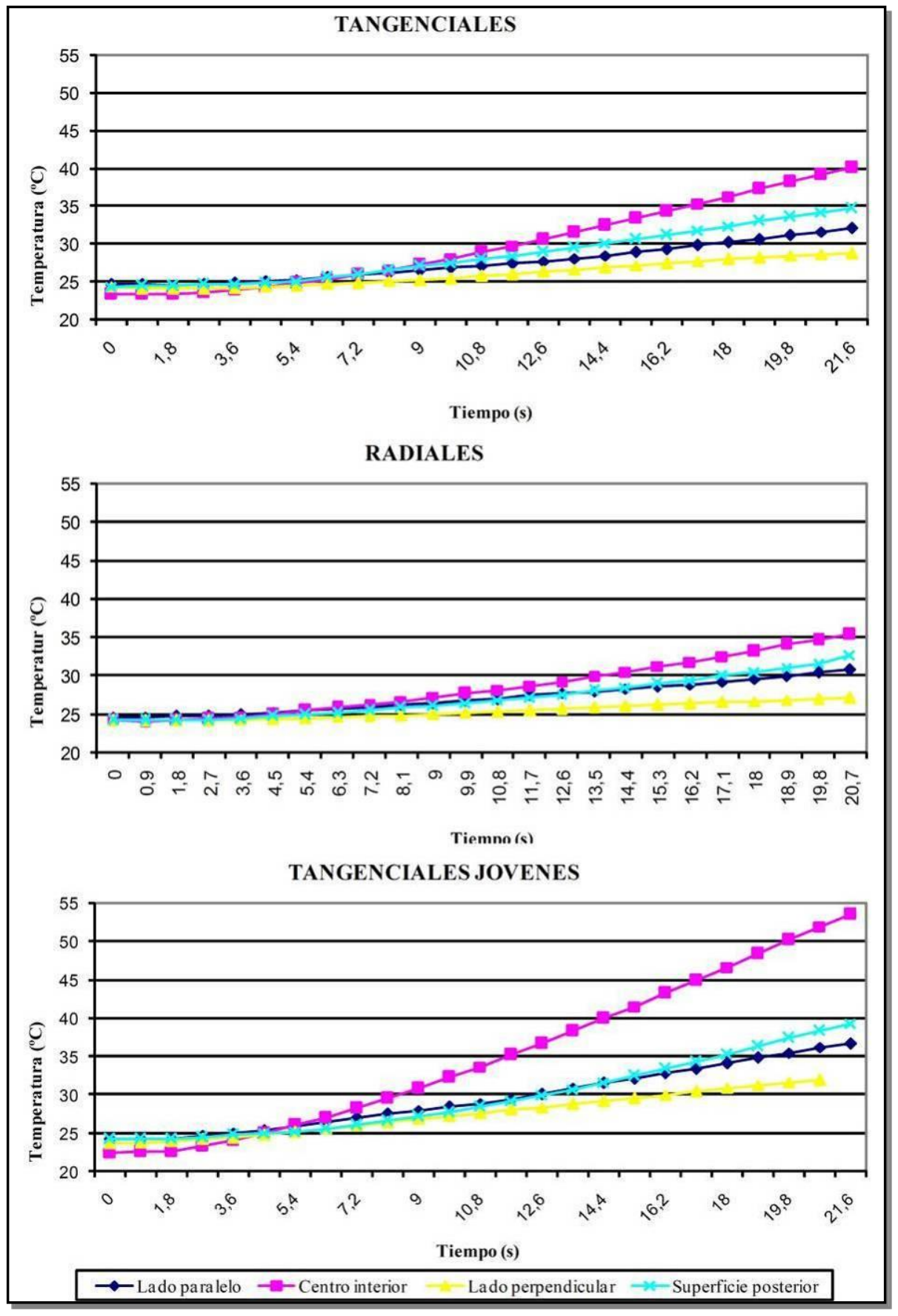

Figura 4-48. Medianas de temperatura en aplicación central

118 Tecnología de microondas en el sector de restauración de bienes culturales 
La Tabla 4-11 muestra la comparación entre las temperaturas máximas alcanzadas por el mismo tratamiento, pero con diferente tipo de polarización en el aplicador.

Tabla 4-11. Temperaturas Máximas Aplicación central

\begin{tabular}{||c|c|c|c|c|c||}
\hline \multirow{2}{*}{ MUESTRA } & \multirow{2}{*}{ ANTENA } & \multicolumn{4}{|c||}{ TEMPERATURA } \\
\cline { 3 - 6 } & & $\begin{array}{c}\text { Interior } \\
{ }^{\mathbf{o}} \mathbf{C}\end{array}$ & $\begin{array}{c}\text { Superficie } \\
{ }^{\mathbf{o}} \mathbf{C}\end{array}$ & $\begin{array}{c}\text { Paralelo } \\
{ }^{\mathbf{o}} \mathbf{C}\end{array}$ & $\begin{array}{c}\text { Perpendicular } \\
{ }^{\mathbf{0}} \mathbf{C}\end{array}$ \\
\hline \multirow{2}{*}{ Tangencial } & Lineal & 50,32 & 36,78 & 31,64 & - \\
\cline { 3 - 7 } & Circular & 38,10 & 33,5 & 31,1 & 28,4 \\
\hline \multirow{2}{*}{ Radial } & Lineal & 47,23 & 37,98 & 31,96 & - \\
\cline { 2 - 7 } & Circular & 34,55 & 31,55 & 30,35 & 29,95 \\
\hline $\begin{array}{c}\text { Tangencial } \\
\text { Joven }\end{array}$ & Circular & 50,15 & 37,3 & 35,45 & 31,25 \\
\hline
\end{tabular}

La temperatura de los puntos centrales disminuye con el aplicador circular y la temperatura en el lado paralelo es casi igual para los dos tipos de aplicador. La temperatura en el lado paralelo también es más cercana al valor de la superficie y al del lado perpendicular, pudiéndose afirmar que el calentamiento logrado en la madera es mucho más homogéneo con la antena de polarización circular que el conseguido con la antena de polarización lineal. La similitud en los valores de temperatura de la superficie y los lados es válida para todos los tipos de muestra.

Por otro lado, el hecho de que exista un diferencia de las temperaturas del lado paralelo y perpendicular muy pequeña indica que la orientación del campo eléctrico respecto a las fibras tiene menos incidencia sobre el calentamiento de la madera. Sin embargo, que el calentamiento percibido no sea totalmente circular es coherente debido a la no homogeneidad de la madera en relación con la orientación de las fibras.

\subsubsection{Temperatura en aplicación solapada}

Los valores de la mediana de temperatura se muestran en la Figura 4-49 . En el caso de las tablas radiales son válidas cuatro muestras, debido al movimiento del centro de la aplicación en una de las muestras; en la primera muestra de las jóvenes la sonda del punto central no funciona, por lo que se cuenta con cuatro muestras también para este punto.

La tendencia de calentamiento es igual para todos los tipos de tablas, aunque nuevamente las tablas tangenciales se calientan más que las radiales y las jóvenes más que las antiguas.

En cuanto a la validez de los datos, el índice de dispersión está por debajo de 9\% para los tres tipos de muestra (Apéndice VI); a excepción del punto perpendicular en tablas jóvenes. Dicho hecho se puede explicar por la cercanía del punto 
perpendicular con el límite de la muestra (vea Figura 4-46), pudiendo afirmar que las microondas interactúan con la misma por la cara de radiación y por el lateral, y que siendo el aire un mal conductor de calor este se acumula en los límites de la muestra.

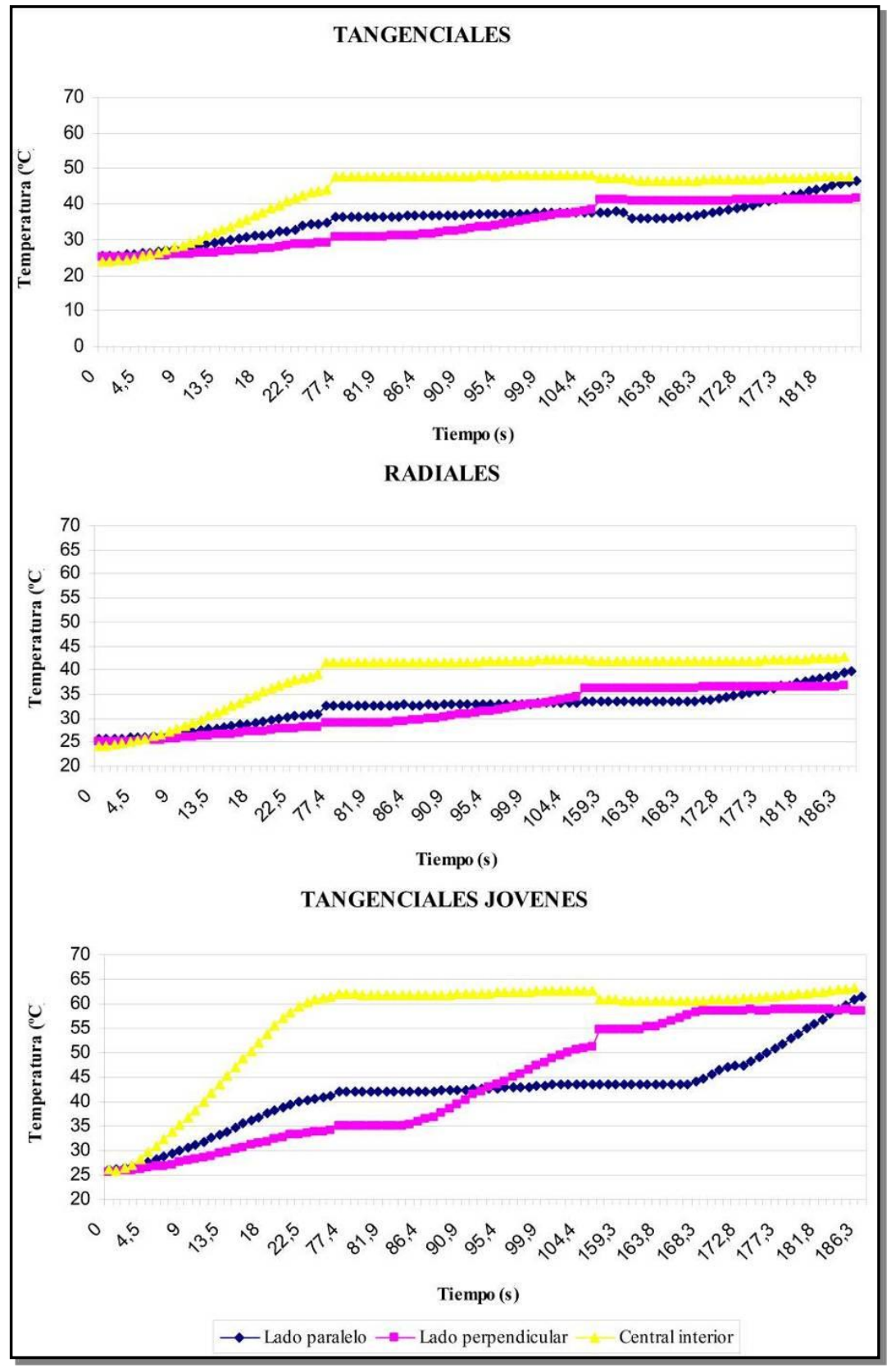

Figura 4-49. Mediana de temperatura aplicación solapada

$\overline{120}$ Tecnología de microondas en el sector de restauración de bienes culturales 
La cercanía del punto perpendicular con el límite de la muestra afecta también el calentamiento en el punto central durante la segunda radiación, lo que no pasa con el punto paralelo en la tercera radiación. En conclusión, los extremos de las piezas a tratar se calientan más que el resto de la pieza si ambos puntos (central y periférico) son expuestos al mismo tratamiento.

En general, la temperatura máxima en el interior de las maderas antiguas no supera los $50{ }^{\circ} \mathrm{C}$ (por debajo del límite de $60^{\circ} \mathrm{C}$ ) y la temperatura alcanzada en los puntos periféricos (o solapados) supera $\operatorname{los} 40^{\circ} \mathrm{C}$ sin alcanzar la temperatura central.

En la Tabla 4-12 se compraran los valores máximos de temperatura alcanzados en diferentes puntos de la muestra en función del tipo de aplicación. La temperatura en el centro de la muestra es la misma para las tablas antiguas, pero para las jóvenes su valor aumenta debido a la influencia que ejerce la segunda radiación sobre el punto central.

En relación con los puntos periféricos, la temperatura aumenta notablemente de la aplicación central a la solapada, haciendo el calentamiento en el interior de la madera más homogéneo, lo que se confirma viendo la cercanía de sus valores de temperatura con los del punto central.

Tabla 4-12.Comparación temperatura entre tipo de aplicación

\begin{tabular}{|c|c|c|c|}
\hline TIPO TABLA & PUNTO & $\begin{array}{c}\text { APLICACIÓN } \\
\text { CENTRAL } \\
\left({ }^{\circ} \mathrm{C}\right)\end{array}$ & $\begin{array}{c}\text { APLICACIÓN } \\
\text { SOLAPADA } \\
\left({ }^{\circ} \mathrm{C}\right)\end{array}$ \\
\hline \multirow{4}{*}{ TANGENCIAL } & Centro Interior & 40 & 40,8 \\
\hline & Superficie posterior & 34 & - \\
\hline & Paralelo Interior & 31,95 & 46,6 \\
\hline & Perpendicular Interior & 28 & 41,87 \\
\hline \multirow{4}{*}{ RADIAL } & Centro Interior & 35,3 & 36,82 \\
\hline & Superficie posterior & 32,65 & - \\
\hline & Paralelo Interior & 30,7 & 39,72 \\
\hline & Perpendicular Interior & 27,1 & 36,7 \\
\hline \multirow{4}{*}{$\begin{array}{c}\text { TANGENCIAL } \\
\text { JOVEN }\end{array}$} & Centro Interior & 53,4 & 58,37 \\
\hline & Superficie posterior & 39,3 & - \\
\hline & Paralelo Interior & 36,65 & 61,6 \\
\hline & Perpendicular Interior & 31,9 & 58,65 \\
\hline
\end{tabular}

En comparación con la antena de polarización lineal (Tabla 4-13), las temperaturas en el centro de la muestra son inferiores con la polarización circular, pero cabe 
resaltar que la diferencia de temperatura entre los dos puntos ubicados en el centro de la muestra es mucho menor, lo que indica una mayor homogeneidad en el calentamiento de la madera.

Esta mayor homogeneidad en el calentamiento también se observa en los puntos periféricos cuando se hace una aplicación solapada, ya que la diferencia de temperatura entre ambos puntos es menor con la polarización circular. El hecho de que las temperaturas alcanzadas en los puntos periféricos para los dos casos sean parecidas, se debe a que estos puntos en los experimentos de polarización lineal están $5 \mathrm{~mm}$ más cerca del centro de la muestra en comparación con los de polarización circular donde están ubicados a $4 \mathrm{~cm}$.

Tabla 4-13. Comparación con polarización lineal

\begin{tabular}{||c|l|c|c||}
\hline \multirow{4}{*}{ TIPO TABLA } & \multicolumn{1}{|c|}{ PUNTO } & $\begin{array}{c}\text { POLARIZACIÓN } \\
\text { LINEAL } \\
\left({ }^{\mathbf{0}} \mathbf{C}\right)\end{array}$ & $\begin{array}{c}\text { POLARIZACIÓN } \\
\text { CIRCULAR } \\
\left({ }^{\mathbf{0}} \mathbf{C}\right)\end{array}$ \\
\hline \multirow{3}{*}{ TANGENCIAL } & Centro Interior & 52,32 & 40 \\
\cline { 2 - 5 } & Sup. Posterior & 36,78 & 34 \\
\cline { 2 - 5 } & Paralelo Interior & 47,20 & 46,6 \\
\cline { 2 - 5 } & Perpendicular & 39,78 & 41,87 \\
\hline \multirow{2}{*}{ RADIAL } & Centro Interior & 47,23 & 35,3 \\
\cline { 2 - 5 } & Sup. Posterior & 37,98 & 32,65 \\
\hline \hline
\end{tabular}

\subsubsection{Efecto del calentamiento}

En la Figura 4-50 se puede observar el efecto del sobrecalentamiento en una muestra irradiada 12 veces con un tratamiento de $1 \mathrm{~kW}$ durante 20 segundos, es decir cerca de 4 minutos de exposición a microondas. Dicho efecto consiste en una exudación de resina sobre las caras de la madera, que aunque a efectos estructurales no reporta ningún daño en la muestra tratada, es un efecto no deseado.

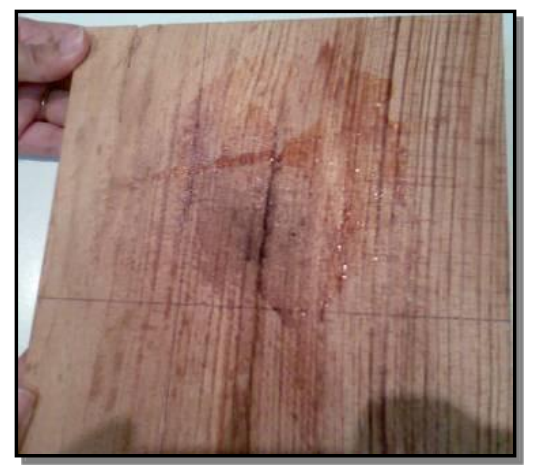

Figura 4-50. Efecto de un tratamiento excesivo sobre la madera

122 Tecnología de microondas en el sector de restauración de bienes culturales 


\subsection{CONCLUSIÓN}

El diseño de un aplicador de microondas que funciona a $2,45 \mathrm{GHz}$ se ha optimizado mediante simulaciones para que tenga polarización circular, parámetros de adaptación alrededor de $-20 \mathrm{~dB}$ y de aislamiento cercanos a $-15 \mathrm{~dB}$. Esto se logra ubicando la alimentación a 20,68 mm del corto con una transición que evita el efecto de puntas, ubicando el plano de apertura del aplicador a $97 \mathrm{~mm}$ del final del septum y colocando una bocina para tener una cobertura de 105 x $105 \mathrm{~mm}$. El diseño final cumple con los requerimientos tanto de la empresa como de los constructores, y es de fácil integración con las demás partes del sistema.

Una vez construido el aplicador se ha verificado su correcto funcionamiento, la polarización circular, los parámetros de adaptación ahora son inferiores a $-13 \mathrm{~dB}$ y el asilamiento superior a $25 \mathrm{~dB}$. Además se han establecidos zonas de seguridad alrededor del aplicador asegurando que los valores de intensidad de campo en dichas zonas no superen los 61,4 V/m para áreas públicas y los $137 \mathrm{~V} / \mathrm{m}$ para áreas de trabajo. Esta última está compuesta por un área en la que el operario puede circular sin traje apantallado y otra en la que debe llevarlo obligatoriamente. En todos los cálculos se tiene en cuenta el peor de los casos posibles, por lo cual los límites son válidos para cualquier tipo de tratamiento con este aplicador.

Se ha comprobado que las temperaturas alcanzadas con el mismo tratamiento del capítulo anterior no representan riesgo para la madera, tanto en el interior como en la superficie. En cuanto al calentamiento, se ha comprobado que con el aplicador de polarización circular éste es más homogéneo y amplio que el alcanzado con el aplicador lineal. También se puede afirmar que el calentamiento con una aplicación solapada es mucho más homogéneo que con aplicación central.

Las zonas de seguridad para piezas grandes deben establecerse desde el centro del aplicador cuando se tratan los bordes de la pieza, y debe analizarse cuidadosamente el tratamiento de dichos bordes ya que se calientan más que el resto de la pieza si son expuestos al mismo tratamiento. Por otro lado aunque las zonas de seguridad estén establecidas en la dirección de máxima radiación se recomienda no situarse enfrente del aplicador durante un tratamiento. 


\section{CONCLUSIONES}

Se ha demostrado de manera experimental el efecto calorífico diferenciado de las microondas en dos casos prácticos:

$>$ En el sector cárnico, demostrando a nivel de laboratorio la viabilidad de erradicar los ácaros de los jamones curados, manteniendo los últimos a temperaturas inferiores a $40{ }^{\circ} \mathrm{C}$.

$>$ En el sector de restauración de bienes culturales, consiguiendo un procedimiento de aplicación inicial para la eliminación de la carcoma en tablas de madera mientras se respetan los límites de temperatura seguros para obras de arte.

El buen funcionamiento de los grupos multidisciplinares formados ha permitido el éxito de los objetivos planteados en cada proyecto, dando una solución no destructiva, higiénica, saludable y respetuosa con el medio ambiente para la problemática planteada en cada sector.

Los resultados encontrados en esta investigación sientan la base para presentar una solución innovadora que haga frente a los problemas de plagas en el sector cárnico y de restauración de bienes culturales dentro del contexto español, acercando estos resultados a la industria y trabajando con ella para que la innovación llegue al mercado.

En relación con los objetivos específicos planteados se tiene que:

1. Las altas perdidas del jamón curado y el hecho que éstas sean mayores en la superficie debido al proceso de absorción de sal garantizan el tratamiento superficial cuando se aplican microondas.

2. Se encontró que con exposiciones prolongadas a bajas potencias de microondas se salvaguarda el jamón mientras se erradican los ácaros

3. Se ha encontrado un procedimiento inicial con una efectividad del $72 \%$. Este procedimiento consta de un tratamiento solapado de 20 segundos y una potencia de microondas de $900 \mathrm{~W}$.

4. Se ha diseñado un aplicador de microondas a $2,45 \mathrm{GHz}$ con polarización circular, los parámetros de adaptación inferior a $-13 \mathrm{~dB}$ y el asilamiento superior a $25 \mathrm{~dB}$. Además se han establecidos las zonas de seguridad alrededor del aplicador. Adicionalmente, se ha comprobado que el calentamiento con el aplicador de polarización circular es más homogéneo y amplio. 


\section{LÍNEAS FUTURAS}

La aplicación de la tecnología de microondas en los sectores planteados está en fases diferentes, debido a la complejidad de los materiales y a la disponibilidad de los estudios previos realizados tanto dentro como fuera del grupo GEA.

\section{EN EL SECTOR CÁRNICO}

El trabajo en ésta área continuará en:

$>$ Realizar experimentos que permitan llevar los resultados obtenidos en el laboratorio a una escala industrial. Actualmente la asociación cuenta con una Ayuda Torres Quevedo para el personal que lleva a cabo esta investigación.

$>$ Estudiar la viabilidad técnica para el diseño de un aplicador prototipo.

$>$ Hacer el desarrollo industrial del prototipo y verificar su correcto funcionamiento. Para esto será necesario buscar apoyo económico en empresas del sector.

$>$ Establecer protocolos y procedimientos de aplicación.

> Implementación del la solución final propuesta, revisiones y soporte.

Adicionalmente, en base a los resultados obtenidos en el CAPÍTULO 1 se podría abrir una investigación que permita utilizar una técnica reflectométrica para la medida del grosor de la capa de grasa sobre jamones frescos y así facilitar su clasificación antes del proceso de cura.

\section{EN EL SECTOR DE RESTAURACIÓN DE BIENES CULTURALES}

La aplicación de la tecnología de microondas en este sector está en un estadio mucho más avanzado, por lo que el trabajo restante es:

Establecer procedimientos y protocolos de aplicación con el aplicador de polarización circular para una alta efectividad, tanto en tablas como en vigas. Teniendo en cuenta la pieza a tratar, los modos de funcionamiento disponibles y el máximo grosor tratable con el sistema.

$>$ Reducir los límites en la dirección de máxima radiación, haciendo un estudio de la atenuación producida por diferentes tipos y grosores de muestras.

$>$ Completar la investigación con aspectos relevantes para las obras de arte, por ejemplo: diferentes tipos de pintura o la existencia de elementos metálicos. 


\section{LOGROS CIENTÍFICOS}

\section{PATENTE}

Procedimientos para el control de ácaros en un producto alimenticio de origen animal mediante aplicación de microondas.

Universidad Politécnica de Valencia.

Concesión: 28 de Octubre de 2005.

\section{REVISTAS}

Microwave disinfestation of bulk timber.

Journal of Microwave Power and Electromagnetic Energy.

International Microwave Power Institute.

Establecimiento de áreas de seguridad para el tratamiento de madera con microondas.

Revista Itekne.

Universidad Santo Tomás.

\section{APORTES EN CONGRESOS}

Initial procedure to exterminate woodworms in panel paintings by microwavepower application.

International Microwave Power Institute's 40th annual Symposium.

August 9-11, 2006. Boston, USA.

Microwave treatment for woodworm disinfection in large-format works of art.

International Conference on Heritage, Weathering and Conservation (HWC-2006). June 21-24, 2006. Madrid, Spain.

Abstract: Microwave treatment for woodworm disinfection in large-format works of art.

Book of abstracts, HWC-2006.

June 21-24, 2006. Madrid, Spain. 


\section{REFERENCIAS}

[1] R. V. Decareau, Microwaves in the food processing industry. Orlando, FL: Academic Press, Inc., 1985.

[2] C. Vicent, B. Panneton, and F. Fleurat-Lessard, Physical control methods in plant protection. Paris: Springer / INRA, 2001.

[3] S. O. Nelson, J. P. G. Bartley, and K. C. Lawrence, "RF and Microwave dielectric properties of stored-grain insects and their implications for potential insect control.," Transactions of the ASAE., vol. 41 (3), pp. 685692, 1998

[4] S. Wang and J. Tang, "Radio frequncy and microwavealternative treatments for insects control in nuts: a review," Agricultural Enguneering Journal, pp. 105-120, 2001.

[5] A. K. Datta and R. C. Ananheswaran, Handbook of microwave technology for food applications. New York: Marcel Dekker, Inc., 2001.

[6] M. V. Hage-Hamsten and S. G. Johansson, "Storage mites," Experimental and Applied Acarology, vol. 16 (1,2), pp. 117-128, 1992.

[7] J. Aranu, M. Hugas, and J. M. Monfort, El jamón curado: aspectos técnicos. Barcelona: Generalitat de Cataluña e Institut de Recerca i Tecnologia Agroalimentaries. , 1988.

[8] J. Arnau and L. Guerrero, "Physical methods of controlling mites in dry cured ham," Fleischwirtschaft, vol. 74 (12), pp. 1311-1313, 1994.

[9] J. Sanchez, "Control de acaros contamienantes del jamón ibérico " in Facultad de Veterinaria. vol. Doctor Extremadura: Universidad de Extramadura, 2002.

[10] M. Escudero and A. López, Etología aplicada al control de las plagas de ácaros del jamón. Sevilla Consejería de Agricultura y Pesca, 2001

[11] M. Calderon and R. Barkai-Golan, Food preservation by modified atmospheres. Boca Raton: CRC Press, 1990.

[12] N. Mori, Y. Kuwahara, and K. Kurosa, "Chemical ecology of astigmatid mites -XLV. (2R,3R)- Epoxyneral: Sex pheromone of the acarid mite Caloglyphus sp. (acarina: acaridae)," Bioorganic \& Medicinal Chemistry, vol. 4, pp. 289-295, 1996.

[13] J. Jorrin, M. Magallanes, and P. Vargas, "Etiología de la peste por ácaros del jamón curado," Eurocarne, vol. 44, pp. 39-44, 2001. 
[14] ASTM, "Standard test methods for complex permitivity of solid electrical insulating materials at microwave frequencies and temperatures to $1650^{\circ} \mathrm{C}$." vol. D2520D, 2001.

[15] A. C. Metaxas and R. J. Meredith, Industrial Microwave Heating. London, United Kingdom: Peter Peregrinus Ltd., 1993.

[16] J. M. Catalá, "Estudio de estructuras guiadas monomodo para aplicaciones de caracterización dieléctrica de materiales y curado de compuestos elastoméricos a frecuencias de microondas," in Departamento de Comunicaciones. vol. Doctor en Telecomunicaciones Valencia: Universidad Politécnica de Valencia, 1999.

[17] A. J. Canos, J. M. Catala-Civera, F. L. Peñaranda-Foix, J. Monzo-Cabrera, and E. De los Reyes, "A new empirical method for extracting unloaded resonant frequencies from microwave resonant cavities," in Microwave Symposium Digest, 2003 IEEE MTT-S International. vol. 3, 8-13 June 2003, pp. 1823-1825.

[18] A. J. Estin and H. E. Bussey, "Errors in dielectric measurements due to a samples insertion hole in a cavity," IEEE Trans. Microwave Theory and Techniques, vol. MTT-8, pp. 650-653, 1960.

[19] H. Kawabata, H. Tanpo, and Y. Kobayashi, "An Improved perturbation method using TM010 mode cylindrical cavity," in Microwave Conference, Asia-Pacific, 2002, pp. 1007-1010.

[20] H. Kawabata, H. Tanpo, and Y. Kobayashi, "A rigorous analysis of a TM010 mode cylindrical cavity tomeasure accurate complez permitivity of liquid," in 33rd European Microwave Conference Munich, 2003.

[21] A. M. Nicholson and G. Ross, "Measurement of intrinsic properties of materials by time domain techniques," IEEE Transactions on Instrumentation and Measurement, vol. 19, pp. 377-382, 1970.

[22] D. Sanchez, J. V. Balbastre, and J. M. Osca, "Microwave Energy as a Viable Alternative to Methyl Bromide and other Pesticides for Rice Disinfection," in Intenational Conference on Alternatives to Methyl Bromide, 2002.

[23] V. R. Lewis and M. I. Haverty, "Evaluation of six techniques for control of the Western drywood termite (Isoptera: Kalotermitidae) in structures," Journal of economic Entonology, vol. 89, pp. 922-934, 1996.

[24] N. Knut, Manual de restauración de cuadros. Barcelona: Könemann, 1999.

[25] H. H. Webber, "High frequency electric fields as lethal agents for insects," Journal of Economic Entomology, pp. 487-498, 1946.

[26] S. O. Nelson, " Review and assessment of radiofrequency and microwave energy for stored-grain insect control," Transactions of the ASAE., vol. 39(4), pp. 1475-1484, 1996. 
[27] S. L. Halverson, "High-power microwave radiation as an alternativa insect control method for store product.," Journal of Economic Entomology, pp. 1638-1648, 1996.

[28] A. Díaz-Morcillo, P. J. Plaza-González, J. V. Balbastre, J. M. Osca, and D. Sánchez-Hernández, "Commercial Rice Disinfection Unit Using Microwave Energy," in Proceedings of IX International Conference on Microwave and high frequency heating Loughborough (England), 2003.

[29] D. Andreuccetti, M. Bini, and A. Ignesti, "Microwave destruction of woodworms," Journal Microwave Power and Electromagnetic Energy, vol. 29 (3), 1994.

[30] D. Andreuccetti, M. Bini, A. Ignesti, A. Gambetta, and R. Olmi, "Feasibility of Microwave Disinfestation of Wood," in Proceedings of 26th Annual Meeting IRG/WP, Helsingor (Denmark), 1995.

[31] D. Andreuccetti, M. Bini, A. Ignesti, R. Olmi, S. Priori, A. Gambetta, and R. Vanni, "A microwave device for woodworm disinfestations," in Proceedings of the International Conference on Microwave and High Frequency Heating, ST John's College, Cambridge(UK), 1995.

[32] M. Bini and D. Andreuccetti, "A portable microwave system for woodworm disinfestations of artistic painted boards," Journal Microwave Power and Electromagnetic Energy, vol. 32(3), pp. 180-187, 1997.

[33] M. Bini, D. Andreuccetti, and A. Ignesti, "Treatment planning in microwave heating of painted wooden boards," in Proceedings of VI International Conference on Microwave and High Frequency Heating, Fermo 1997, pp. 361-364.

[34] ICNRP, "Guidelines for limiting exposure to time varying electric, magnetic and elecnafetic fields (as high as $300 \mathrm{GHz}$ )." I. C. o. N. R. Protection, Ed., 1998

[35] CE, "Directiva 2004/04/CE," P. Europeo, Ed., 2004.

[36] M. Presidencia, "REAL DECRETO 1066/2001 de 28 de septiembre. Reglamento que establece condiciones de protección del dominio público radioeléctrico, restricciones a las emisiones radioeléctricas y medidas de protección sanitaria frente a emisiones radioeléctricas.," BOE núm. 234 de 29 de septiembre, 2001, pp. 36217-36227.

[37] M. Norimoto, "Dielectric properties of wood," Wood research, vol. 59, pp. 106-152, 1976.

[38] G. I. Torgovnikov, Dielectric properties of wood and wood-based materials. New York: Springler-Verlag, 1993.

[39] P. J. Plaza, A. T. Zona, R. Sanchís, J. V. Balbastre, A. Fernández, E. M. Muñoz, J. Gordillo, and E. d. 1. Reyes, "Microwave Disinfestation of Bulk Timber," Journal of Microwave Power and Electromagnetic Energy, 2007. 
[40] CEN, "Wood Preservatives - Determination of the toxic values against larvae of Hylotrupes bajulus (Linnaeus) - (Laboratory method)." vol. prEN 47:2003 E: European Committee for Standardization, 2003.

[41] V. H. Heywood, Flowering Plants of the World. London: B.T. Batsford, 1996.

[42] J. M. Romani, Apuntes sobre la madera. Barcelona: Escuela Técnica Superior de Arquitectura, 1974.

[43] C. P. López, Estadistica. Problemas resueltos y aplicaciones. Madrid: Pearson educación, S.A., 2003.

[44] L. Nuño, J. Balbastre, L. Llácer, and H. González, Electródinamica para ingenieros -teoría y problemas-. Valencia: Ediciones VJ, 2005.

[45] C. A. Balanis, Advanced engineering electromagnetics. New York: John Wiley \& Sons, 1989.

[46] CENELEC, "Exposición humana a campos eléctromagneticos." vol. 501662: Norma Europea, 1994. 


\section{ÍNDICE DE TABLAS}

Tabla 1-1. Permitividad de grasa fresca .................................................................. 15

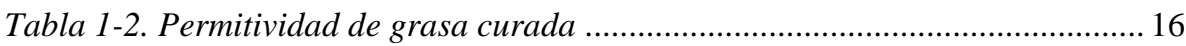

Tabla 1-3. Medidas de permitividad en guía ......................................................... 23

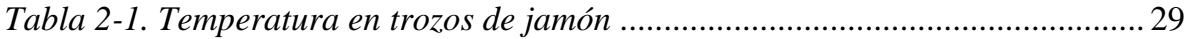

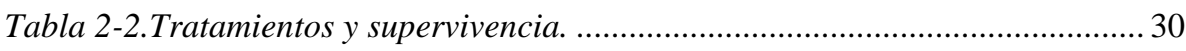

Tabla 2-3. Verificación de tratamientos .............................................................. 31

Tabla 2-4. Temperatura de jamones en valoración sensorial. ................................. 32

Tabla 2-5. Tratamiento piezas enteras .................................................................... 33

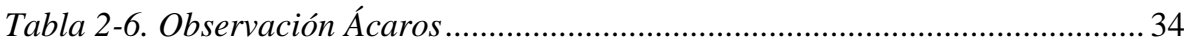

Tabla 2-7. Segundo tratamiento piezas enteras ................................................... 35

Tabla 3-1. Materiales empleados y sus características .......................................... 41

Tabla 3-2. Consideraciones para la selección de los tratamientos .......................... 45

Tabla 3-3. Tablas preparadas con imprimación .................................................... 47

Tabla 3-4. Tiempos para tratamiento inicial....................................................... 50

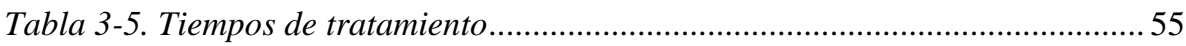

Tabla 3-6. Tasa de mortalidad con aplicación central ..........................................5 56

Tabla 3-7. Tiempos empleados en modelos con solape .......................................... 59

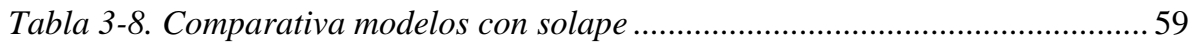

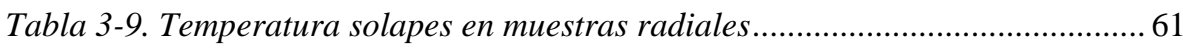

Tabla 3-10. Temperatura solapes en muestras tangenciales................................. 61

Tabla 3-11. Tasa de mortalidad con radiación solapada ........................................ 64

Tabla 3-12. Temperatura pruebas de estructura ................................................... 65

Tabla 3-13. Variaciones pruebas de estructura .................................................... 65

Tabla 3-14. Temperaturas máximas pruebas imprimación ...................................... 65

Tabla 3-15. Comparación de temperaturas pruebas imprimación ........................... 68

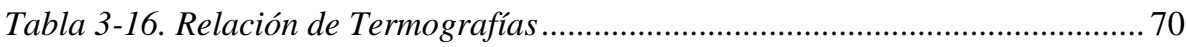


Tabla 4-1. Comparación ampliación con y sin Ridge ………………………........ 101

Tabla 4-2. Comparación ampliación con Ridge y con Bocina ................................. 104

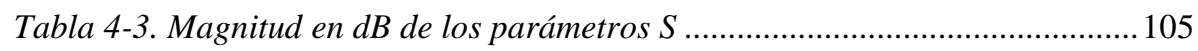

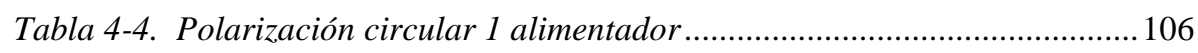

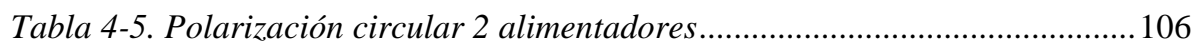

Tabla 4-6.Radios de seguridad- superficie irradiada .............................................110

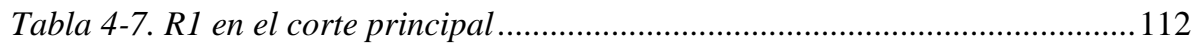

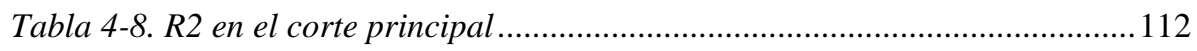

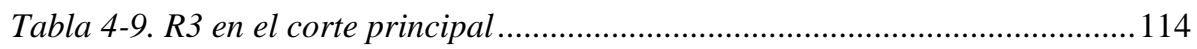

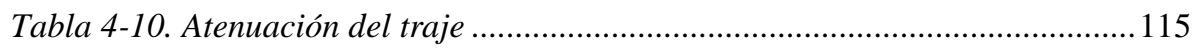

Tabla 4-11. Temperaturas Máximas Aplicación central ..........................................119

Tabla 4-12.Comparación temperatura entre tipo de aplicación ..............................121

Tabla 4-13. Comparación con polarización lineal ................................................. 122 


\section{ÍNDICE DE FIGURAS}

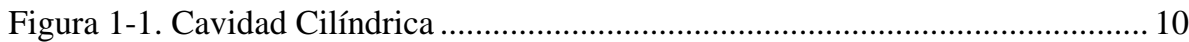

Figura 1-2. Software para el cálculo de la permitividad.......................................... 11

Figura 1-3. Configuración para la medida de permitividad......................................... 11

Figura 1-4. Permitividad - Frecuencia y Q de origen diferente ................................. 12

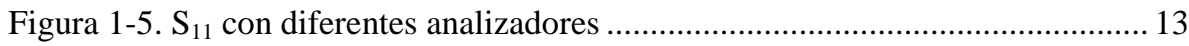

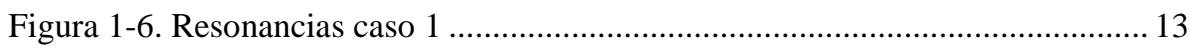

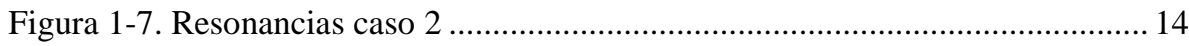

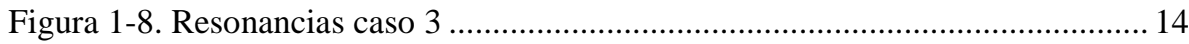

Figura 1-9. Medidas de permitividad - grasa fresca ……………………............... 15

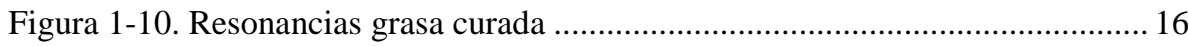

Figura 1-11. Medidas de permitividad - grasa curada ............................................. 16

Figura 1-12. Perturbación magro fresco ………………………………………...... 17

Figura 1-13. Frecuencia a 100\% de profundidad................................................... 18

Figura 1-14. Configuración de medida de permitividad en guía. .............................. 22

Figura 1-15. Muestras para medidas en guía ......................................................... 22

Figura 1-16. Permitividad vs. Frecuencia ............................................................ 23

Figura 2-1. Configuración - experimentos en trozos de jamón ................................ 25

Figura 2-2.Muestras verificación del tratamiento ...................................................... 27

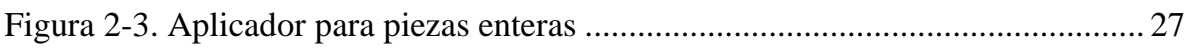

Figura 2-4. Partes de la pieza de jamón .............................................................. 28

Figura 2-5. Ubicación de la sonda en el magro ....................................................... 29

Figura 3-1. Configuración para la experimentación .................................................. 43

Figura 3-2. Fases de la Experimentación................................................................ 43

Figura 3-3. Ubicación de la sondas de temperatura (1 ra fase)................................... 44

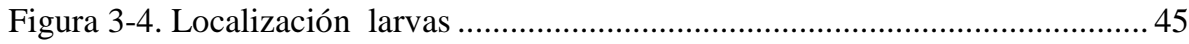

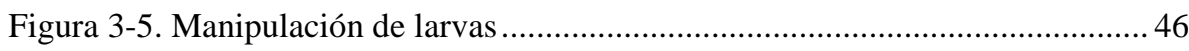


Figura 3-6. Matriz para la medida de movimientos. ................................................. 46

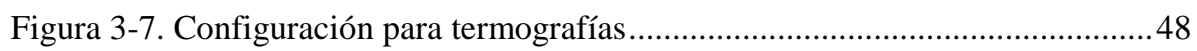

Figura 3-8.Configuración para temperatura interior en listones ................................48

Figura 3-9.Configuración para temperatura superficial en listones ..........................49

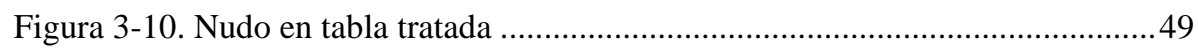

Figura 3-11. Medidas de Temperatura inicial .......................................................51

Figura 3-12. Factor de forma del campo eléctrico del aplicador ...........................52

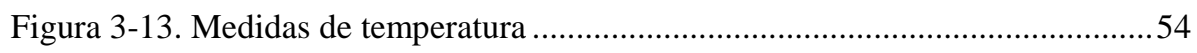

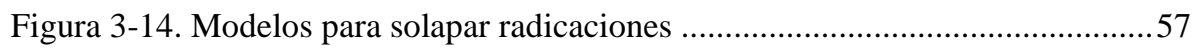

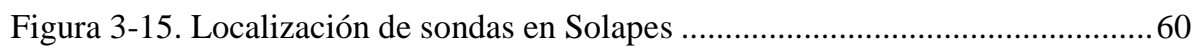

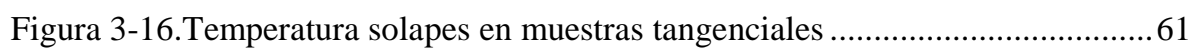

Figura 3-17. Índice de dispersión solapes muestras tangenciales .............................62 62

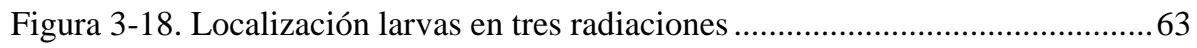

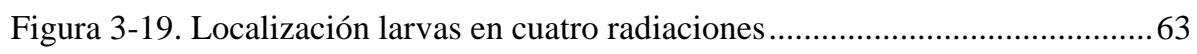

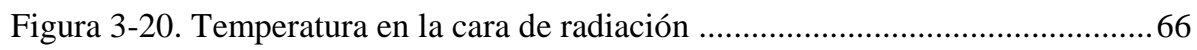

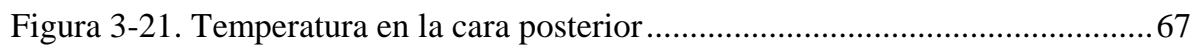

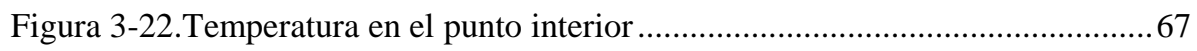

Figura 3-23. Termografías internas muestras con imprimación .............................69

Figura 3-24.Termografías muestras sin imprimación ............................................70

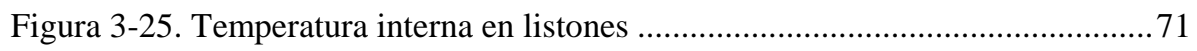

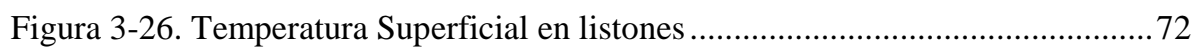

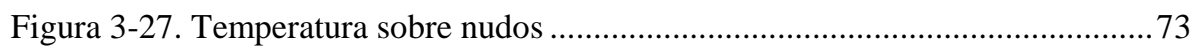

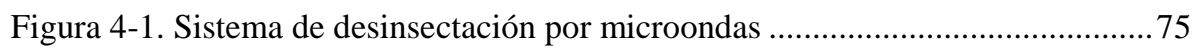

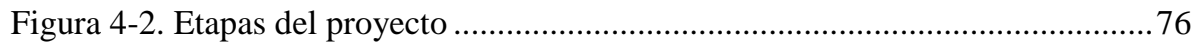

Figura 4-3. Metodología para la optimización ..................................................... 77

Figura 4-4. Configuración para medidas de polarización ........................................79

Figura 4-5. Protocolo medida de Polarización....................................................... 79

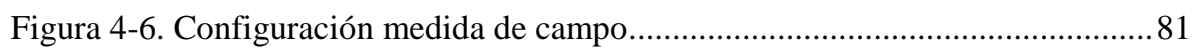

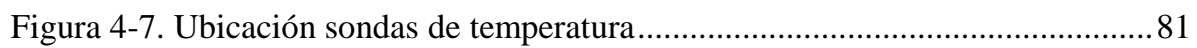

Figura 4-8.Procedimiento experimental medidas de temperatura ........................... 82

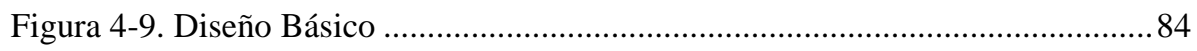


Figura 4-10. Parámetros Polarización modo A - Diseño Básico ............................. 84

Figura 4-11. Parámetros S modo A- Diseño Básico ................................................. 85

Figura 4-12. Parámetros polarización modo B - Diseño Básico.................................. 86

Figura 4-13. Parámetros S modo B- Diseño Básico .............................................. 86

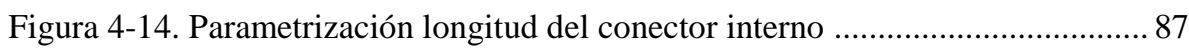

Figura 4-15. Parametrización Inicial - ubicación alimentación ................................. 88

Figura 4-16. Parametrización - ubicación alimentación .......................................... 89

Figura 4-17. Optimización ubicación alimentación - Modo A ................................ 89

Figura 4-18. Relación Axial Ubicación alimentación - Modo A ............................. 90

Figura 4-19. Desfase Ubicación alimentación - Modo A....................................... 91

Figura 4-20. Relación Axial ubicación alimentación - Modo B ............................... 92

Figura 4-21. Desfase ubicación alimentación - Modo B ........................................ 92

Figura 4-22. Diseño Optimizado con transición .................................................... 93

Figura 4-23. Parámetros S con transición - Modo A ............................................. 94

Figura 4-24. Relación axial con transición- Modo A ............................................. 95

Figura 4-25. Desfase con transición- Modo A....................................................... 95

Figura 4-26. Parametrización alimentación con transición- Modo A........................ 96

Figura 4-27. Parámetros S con transición optimizada -Modo A ............................. 96

Figura 4-28. Parametrización de la longitud del aplicador - Modo A ...................... 97

Figura 4-29. Parámetros S diseño ajustado - Modo A............................................... 98

Figura 4-30. Relación axial diseño ajustado -Modo A .......................................... 98

Figura 4-31. Desfase diseño ajustado -Modo A ................................................... 99

Figura 4-32. Ampliación Ridge de la cobertura ..................................................... 100

Figura 4-33. Campo eléctrico ampliación Ridge ................................................ 100

Figura 4-34. Parametrización alimentación ampliación Ridge .............................. 102

Figura 4-35. Ampliación bocina de la cobertura ................................................. 102

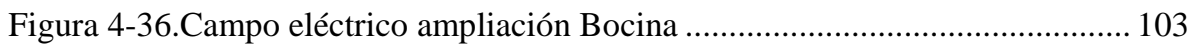

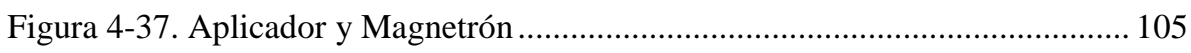

Figura 4-38.Intensidad de Campo Eléctrico en la superficie irradiada -1 kW ........ 108

Figura 4-39. Campo Eléctrico en la superficie irradiada- 2 kW ............................. 109

Figura 4-40. Zonas de seguridad - superficie irradiada .................................... 110 
Figura 4-41. Campo corte principal. .................................................................... 111

Figura 4-42. Medidas con el traje apantallado..................................................... 112

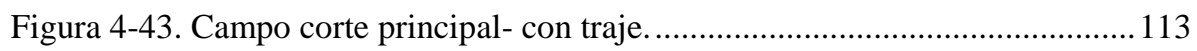

Figura 4-44. Zonas de seguridad - corte principal ............................................... 114

Figura 4-45 . Zona de seguridad completas en superficie irradiada........................ 116

Figura 4-46. Ubicación de sondas muestras tangenciales jóvenes ............................ 116

Figura 4-47. Medidas Temperatura aplicación central ......................................... 117

Figura 4-48. Medianas de temperatura en aplicación central.................................. 118

Figura 4-49 . Mediana de temperatura aplicación solapada...................................... 120

Figura 4-50. Efecto de un tratamiento excesivo sobre la madera ............................ 122 


\section{APÉNDICES}




\section{APÉNDICE I. PERMITIVIDAD DEL JAMÓN}

\section{i. Influencia del tubo sobre la medida}

En la siguiente tabla se muestra la diferencia entre las medidas realizadas con la cavidad vacía y con la cavidad con un tubo introducido al $50 \%$ de la altura de cavidad. La diferencia entre los resultados obtenidos para la frecuencia y el factor de calidad es despreciable.

Tabla 14. Resultados medidas con tubo

\begin{tabular}{|c|c|c|c|}
\hline Medida & Muestra & Frecuencia & Factor Q \\
\hline \hline \multirow{2}{*}{1} & vacio & 2,34 & 167,81 \\
\cline { 2 - 4 } & tubo50\% & 2,34 & 170,51 \\
\hline \multirow{2}{*}{2} & vacía & 2,34 & 153,96 \\
\cline { 2 - 4 } & tubo50\% & 2,34 & 155,04 \\
\hline
\end{tabular}

\section{ii. Medidas de grasa fresca}

La siguiente tabla muestra los resultados obtenidos del programa para cada una de las muestras de grasa fresca medidas con el método de cavidad resonante.

Tabla 15. Muestras de grasa fresca

\begin{tabular}{|c|c|c|}
\hline MUESTRA & $\varepsilon ’$ & $\varepsilon ”$ \\
\hline \multicolumn{3}{|c|}{ Caso 1} \\
\hline grasa_3 & 3,7563 & 1,1318 \\
\hline grasa_4 & 3,6191 & 0,7048 \\
\hline grasa_5 & 3,7151 & 0,5738 \\
\hline grasa_6 & 3,5756 & 0,6067 \\
\hline grasa_7 & 3,2497 & 0,4687 \\
\hline \multicolumn{3}{|c|}{ Caso 2} \\
\hline grasa_1 & 3,5290 & 0,4047 \\
\hline grasa_2 & 3,7149 & 0,4814 \\
\hline grasa_3 & 3,9448 & 0,5902 \\
\hline grasa_4 & 3,8291 & 0,4398 \\
\hline grasa_5 & 3,8348 & 0,5463 \\
\hline grasa_6 & 3,7690 & 0,5613 \\
\hline grasa_7 & 3,7963 & 0,5318 \\
\hline
\end{tabular}




\begin{tabular}{|l|c|c|}
\hline \multicolumn{3}{|c|}{ Caso 3 } \\
\hline grasa_1 & 3,2952 & 0,3845 \\
\hline grasa_2 & 3,5568 & 0,5816 \\
\hline grasa_3 & 3,4665 & 0,5703 \\
\hline grasa_4 & 3,5613 & 0,6008 \\
\hline grasa_5 & 3,5582 & 0,6559 \\
\hline grasa_6 & 3,5555 & 0,6570 \\
\hline grasa_7 & 3,4696 & 0,5428 \\
\hline
\end{tabular}

\section{iii. Medidas de grasa curada}

La siguiente tabla muestra los resultados obtenidos del programa para cada una de las muestras de grasa curada medidas con el método de cavidad resonante.

Tabla 16. Muestras de grasa curada

\begin{tabular}{|c|c|c|}
\hline MUESTRA & $\boldsymbol{\varepsilon}$ & $\boldsymbol{\varepsilon}{ }^{\prime}$ \\
\hline \hline grasa_1 & 2,6871 & 0,2113 \\
\hline grasa_12 & 2,7148 & 0,2735 \\
\hline grasa_4 & 2,7492 & 0,3015 \\
\hline
\end{tabular}




\section{APÉNDICE II. DESISECTACIÓN DE RETABLOS.}

\section{i. Tiempos de duración del tratamiento}

Los tiempos de tratamiento para todos los casos estudiados en las medidas con el punto interior paralelo a $8 \mathrm{~cm}$ se muestran en la Tabla 17, estos tiempos se seleccionan de acuerdo a las restricciones dadas en la Tabla 3-2. Como muestran los datos el caso más restrictivo es con muestras tangenciales cuando el campo esta paralelo a las fibras, ya que la temperatura límite en el punto interior centro es alcanzada más rápidamente, mientras la temperatura en el punto cara posterior se mantiene por debajo del límite fijado para la cara pintada.

Tabla 17. Tiempo de tratamientos inicial - todos los casos

\begin{tabular}{|c|c|c|c|c|c|c|c|c|c|c|}
\hline & \multicolumn{10}{|c|}{ Radiación paralela a las fibras } \\
\hline & \multicolumn{5}{|c|}{ Tangencial } & \multicolumn{5}{|c|}{ Radial } \\
\hline & \multicolumn{3}{|c|}{ Interior centro } & \multicolumn{2}{|c|}{ Cara posterior } & \multicolumn{3}{|c|}{ Interior centro } & \multicolumn{2}{|c|}{ Cara posterior } \\
\hline & \begin{tabular}{c|}
$\mathbf{t}$ \\
$(\mathbf{s})$
\end{tabular} & $\begin{array}{c}\text { Med. } \\
{ }^{\circ} \mathrm{C}\end{array}$ & $\begin{array}{l}\mathbf{T} . \\
{ }^{\mathbf{o}} \mathbf{C}\end{array}$ & $\begin{array}{c}\text { Med. } \\
{ }^{\circ} \mathrm{C}\end{array}$ & $\begin{array}{c}\mathbf{T}+\mathbf{2} \\
{ }^{\circ} \mathrm{C}\end{array}$ & $\mathbf{t}(\mathbf{s})$ & $\begin{array}{l}\text { Med. } \\
{ }^{\circ} \mathrm{C}\end{array}$ & $\begin{array}{l}\mathbf{T} . \\
{ }^{\mathbf{o}} \mathbf{C}\end{array}$ & $\begin{array}{c}\text { Med. } \\
{ }^{\circ} \mathrm{C}\end{array}$ & $\begin{array}{c}\mathbf{T}+\mathbf{2} \\
{ }^{\circ} \mathrm{C}\end{array}$ \\
\hline $1 \sigma$ & 14,4 & 49,3 & 56,71 & 34,8 & 48,03 & 19,2 & 56,37 & 60,91 & 33,65 & 43,80 \\
\hline $2 \sigma$ & 12 & 44,24 & 57,40 & 32,08 & 43,05 & 16,8 & 52,04 & 59,89 & 31,7 & 41,11 \\
\hline \multirow[t]{5}{*}{$3 \sigma$} & 9,6 & 39,1 & 55,85 & 29,02 & 37,52 & 14,4 & 47,77 & 58,13 & 29,76 & 37,90 \\
\hline & \multirow{2}{*}{\multicolumn{10}{|c|}{ Radiación p }} \\
\hline & \multicolumn{2}{|c|}{ Tangencial } & & & & & \multicolumn{4}{|c|}{ Radial } \\
\hline & \multicolumn{3}{|c|}{ Interior centro } & \multicolumn{2}{|c|}{ Cara posterior } & \multicolumn{3}{|c|}{ Interior centro } & \multicolumn{2}{|c|}{ Cara posterior } \\
\hline & $\begin{array}{c}\mathbf{t} \\
(\mathrm{s})\end{array}$ & $\begin{array}{c}\text { Med. } \\
{ }^{\circ} \mathrm{C} \\
\end{array}$ & $\begin{array}{l}\mathbf{T} . \\
{ }^{\mathbf{0}} \mathbf{C}\end{array}$ & $\begin{array}{c}\text { Med. } \\
{ }^{\circ} \mathrm{C} \\
\end{array}$ & $\begin{array}{c}\mathbf{T}+2 \sigma \\
{ }^{\circ} \mathrm{C}\end{array}$ & $\mathbf{t}(\mathbf{s})$ & $\begin{array}{c}\text { Med. } \\
{ }^{\circ} \mathrm{C} \\
\end{array}$ & $\begin{array}{l}\mathrm{T} . \\
{ }^{\mathbf{0}} \mathbf{C}\end{array}$ & $\begin{array}{c}\text { Med. } \\
{ }^{\circ} \mathrm{C}\end{array}$ & $\begin{array}{c}\mathbf{T}+\mathbf{2} \sigma \\
{ }^{\circ} \mathrm{C}\end{array}$ \\
\hline $1 \sigma$ & 21,6 & 52,22 & 55,49 & 38,36 & 41,02 & 24 & 55,1 & 60,99 & 37,46 & 43,28 \\
\hline $2 \sigma$ & 21,6 & 52,22 & 60,77 & 38,36 & 41,02 & 19,2 & 47,56 & 56,92 & 33,9 & 38,58 \\
\hline $3 \sigma$ & 19,2 & 48,04 & 59,62 & 32,24 & 34,81 & 16,8 & 43,6 & 55,77 & 32,08 & 36,3 \\
\hline
\end{tabular}

En la Figura 1 se muestra el índice de dispersión para los cuatro casos. Los valores de temperatura utilizados son la mediana de la muestras y el índice de dispersión es la desviación estándar normalizada con la mediana [43].

Mediana: valor medio de la distribución, suponiendo que esta ordenada de menor a mayor, que deja a su izquierda y a su derecha la misma frecuencia de observaciones. Si el número de muestras es par, se toma como mediana la media aritmética de los dos valores centrales. Es un valor robusto ya que no influyen en ella los valores extremos. Aunque el cálculo se hizo en Excel, su expresión es:

$$
M_{e}=L_{i-1}+\frac{N / 2-N_{i-1}}{n_{i}} c_{i}
$$


Donde: $\mathrm{N}_{\mathrm{i}-1}<\mathrm{N} / 2<\mathrm{N}_{\mathrm{i}},\left[\mathrm{L}_{\mathrm{i}-1}, \mathrm{~L}_{\mathrm{i}}\right]$ es el intervalo siguiente al que contiene a $\mathrm{N} / 2 \mathrm{y}$ $c_{i}$ su amplitud.

Desviación estándar: medida de dispersión absoluta referida a promedios. Es la raíz cuadrada con signo positivo de la varianza, por lo que viene expresada en las mismas unidades que la distribución, lo que la hace más apta como medida de dispersión. Siendo $x_{i}$ muestra número i, $\mathrm{N}$ número total de muestras y $M_{e}$ mediana, su expresión es:

$$
D_{M e}=\sqrt{\frac{1}{N} \sum_{i=1}^{N}\left(x_{i}-M_{e}\right)^{2}}
$$

Índice de dispersión: se usa para resolver el problema de comparación de medianas de varias distribuciones. Se define como la relación por cociente entre la desviación media respecto de la mediana. Evidentemente, cuanto menor sea el índice de dispersión más representativa es la mediana.

$$
V_{M e}=\frac{D_{M e}}{M_{e}}
$$

Los tiempos de tratamiento para los cuatro casos estudiados en las medidas con el punto interior paralelo a $4 \mathrm{~cm}$ se muestran en la Tabla 18. El caso más restrictivo es de nuevo con muestras tangenciales y el campo orientado en dirección paralela a las fibras. La desviación estándar de estas medidas se muestra en la Figura 2.

\begin{tabular}{|c|c|c|c|c|c|c|c|c|c|c|}
\hline & \multicolumn{10}{|c|}{ Radiación paralela a las fibras } \\
\hline & \multicolumn{5}{|c|}{ Tangencial } & \multicolumn{5}{|c|}{ Radial } \\
\hline & \multicolumn{3}{|c|}{ Centro Interna } & \multicolumn{2}{|c|}{ Centro posterior } & \multicolumn{3}{|c|}{ Centro Interna } & \multicolumn{2}{|c|}{ Centro posterior } \\
\hline & \begin{tabular}{|c|}
$\mathbf{t}$ \\
$(\mathrm{s})$
\end{tabular} & \begin{tabular}{|c|} 
Med. \\
${ }^{\circ} \mathrm{C}$
\end{tabular} & $\begin{array}{l}\mathbf{T} . \\
{ }^{\circ} \mathbf{C} \\
\end{array}$ & \begin{tabular}{c|}
$\mathrm{T}$ \\
${ }^{\circ} \mathrm{C}$ \\
\end{tabular} & $\begin{array}{c}\mathrm{T}+2 \sigma \\
{ }^{\circ} \mathrm{C} \\
\end{array}$ & \begin{tabular}{|c|}
$\mathbf{t}$ \\
$(\mathrm{s})$
\end{tabular} & $\begin{array}{c}\text { Med. } \\
{ }^{\circ} \mathrm{C} \\
\end{array}$ & $\begin{array}{l}\mathbf{T} . \\
{ }^{\mathbf{0}} \mathbf{C}\end{array}$ & \begin{tabular}{c|}
$\mathrm{T}$ \\
${ }^{\circ} \mathrm{C}$ \\
\end{tabular} & $\begin{array}{c}\mathbf{T}+2 \sigma \\
{ }^{\circ} \mathrm{C}\end{array}$ \\
\hline $1 \sigma$ & 19,2 & 52,32 & 56,52 & 36,78 & 48,42 & 24 & 55,31 & 59,28 & 43,45 & 53,10 \\
\hline $2 \sigma$ & 16,8 & 45,96 & 56,93 & 34,08 & 44,48 & 21,6 & 51,27 & 58,11 & 40,64 & 49,28 \\
\hline \multirow[t]{5}{*}{$3 \sigma$} & 14,4 & 42,00 & 56,52 & 31,48 & 40,57 & 19,2 & 47,23 & 56,21 & 37,98 & 45,77 \\
\hline & \multicolumn{10}{|c|}{ Radiación } \\
\hline & \multicolumn{5}{|c|}{ Tangencial } & \multicolumn{5}{|c|}{ Radial } \\
\hline & \multicolumn{3}{|c|}{ Centro Interna } & \multicolumn{2}{|c|}{ Centro posterior } & \multicolumn{3}{|c|}{ Centro Interna } & \multicolumn{2}{|c|}{ Centro posterior } \\
\hline & $\begin{array}{c}\mathbf{t} \\
(\mathbf{s})\end{array}$ & \begin{tabular}{|c|} 
Med. \\
${ }^{\circ} \mathrm{C}$
\end{tabular} & $\begin{array}{l}\mathbf{T} . \\
{ }^{\circ} \mathbf{C}\end{array}$ & \begin{tabular}{|c|}
$\mathrm{T}$ \\
${ }^{\circ} \mathrm{C}$ \\
\end{tabular} & $\begin{array}{c}\mathrm{T}+2 \sigma \\
{ }^{\circ} \mathrm{C}\end{array}$ & \begin{tabular}{|c|}
$\mathbf{t}$ \\
$(\mathbf{s})$
\end{tabular} & \begin{tabular}{|c|} 
Med. \\
${ }^{\circ} \mathrm{C}$
\end{tabular} & $\begin{array}{l}\mathbf{T} . \\
{ }^{\circ} \mathbf{C}\end{array}$ & \begin{tabular}{c|}
$\mathrm{T}$ \\
${ }^{\circ} \mathrm{C}$ \\
\end{tabular} & $\begin{array}{c}\mathrm{T}+2 \sigma \\
{ }^{\circ} \mathrm{C}\end{array}$ \\
\hline $1 \sigma$ & 31,2 & 57,00 & 60,38 & 42,52 & 55,18 & 24 & 50,94 & 58,01 & 38,80 & 47,67 \\
\hline $2 \sigma$ & 28,8 & 53,74 & 59,85 & 40,36 & 51,90 & 21,6 & 47,72 & 60,18 & 36,42 & 44,37 \\
\hline $3 \sigma$ & 26,4 & 50,58 & 58,67 & 38,30 & 48,90 & 19,2 & 44,20 & 60,45 & 33,94 & 40,96 \\
\hline
\end{tabular}

Tabla 18. Tiempos de tratamiento - todos los casos 


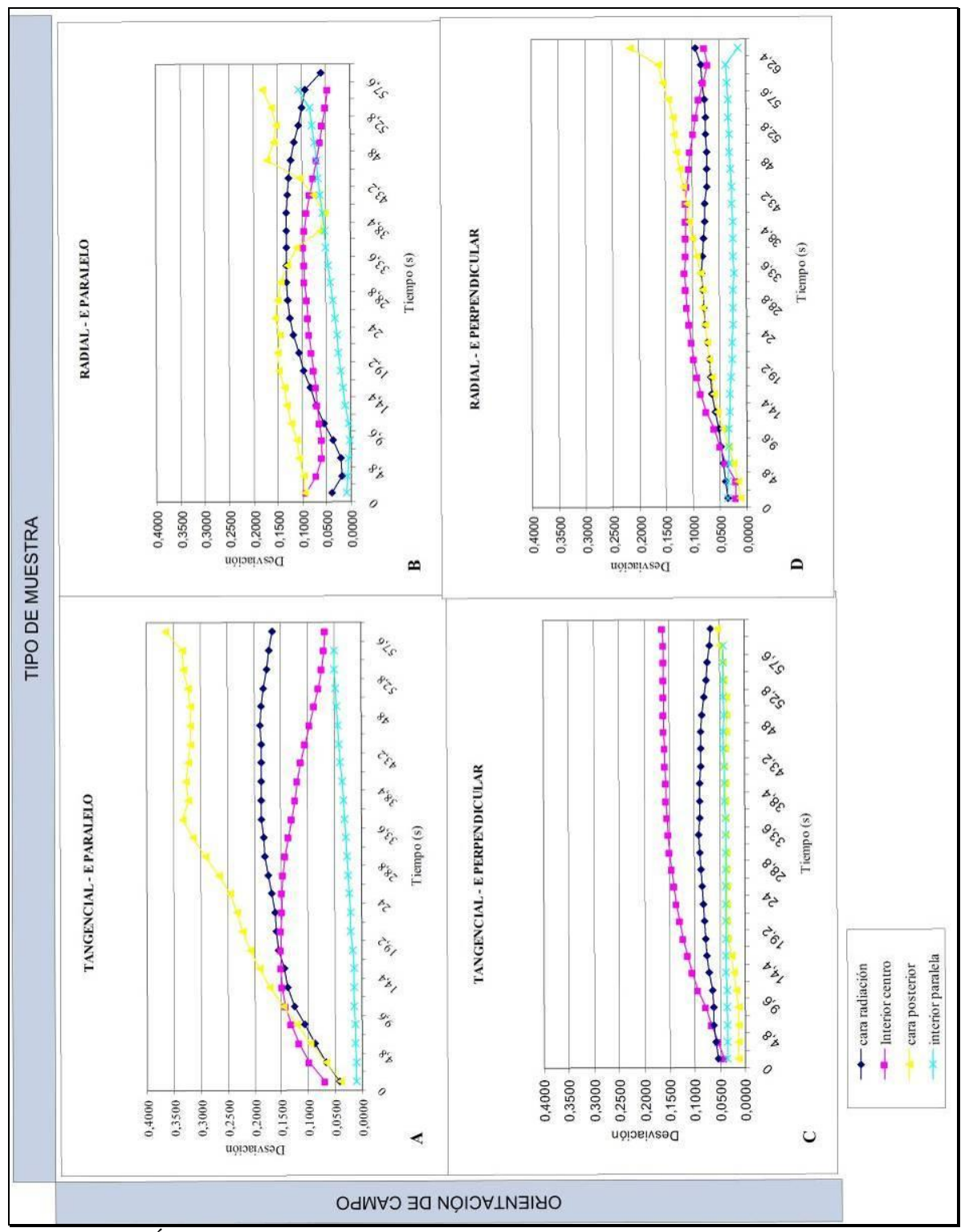

Figura 51. Índice de dispersión respecto a la mediana medidas iniciales de temperatura 


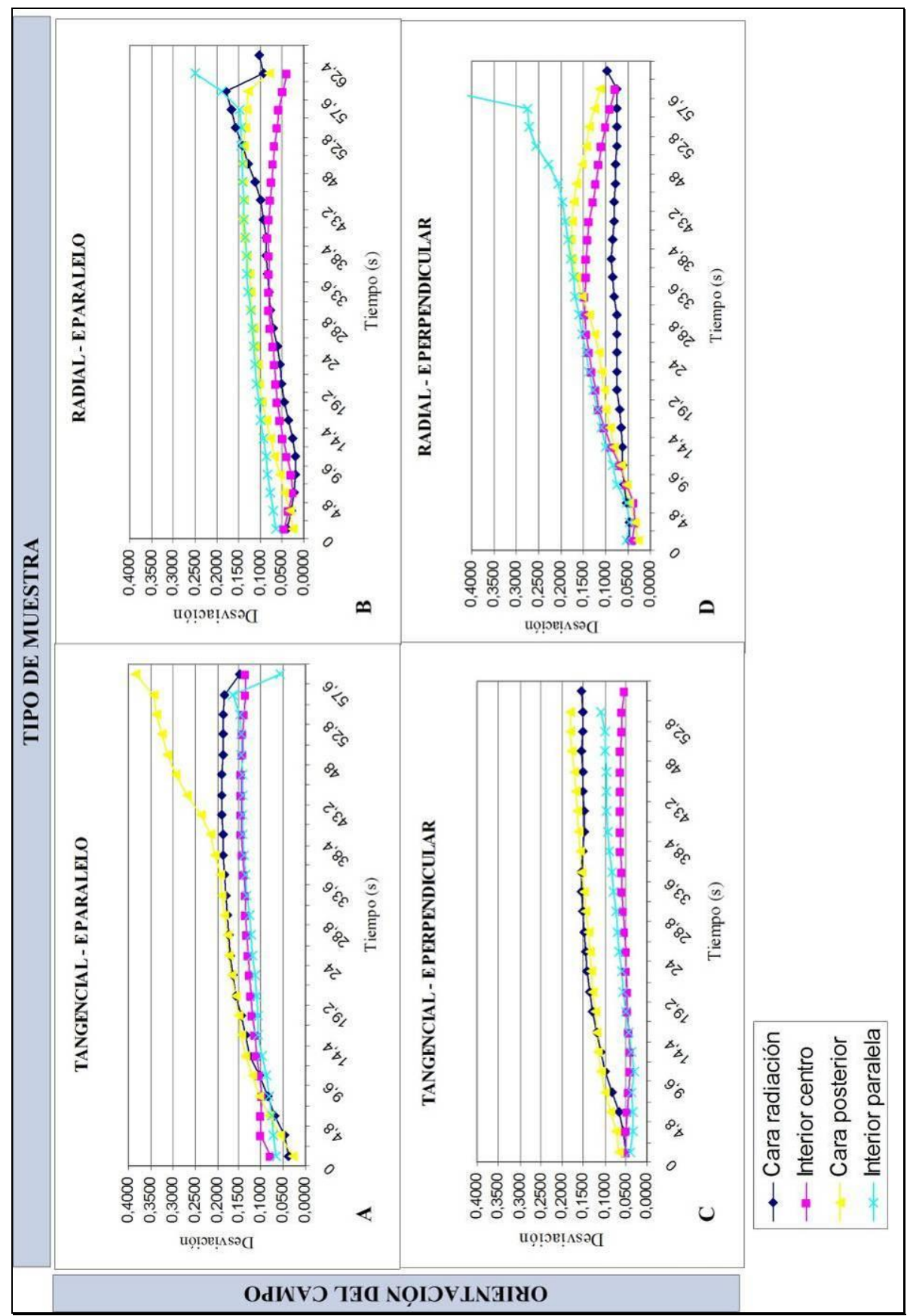

Figura 52. Índice de dispersión respecto a la mediana medidas de temperatura 


\section{ii. Tasa de Mortalidad}

Los datos detallados de la observación para el tratamiento de aplicación central se muestran en las Tablas 6, 7 y 8, clasificadas según el tipo de tratamiento y muestra, La población de control es la misma para todos los tiempos, ya que la experimentación se hizo el mismo día, la observación de esta población se muestra en la Tabla 9. La observación del tratamiento con solapamiento se presenta en las Tablas 10, 12 y 14 y el seguimiento del control para cada experimento en las Tablas 11,13 y 15 .

El código utilizado en las tablas es:

$1=$ MUERTA $/ 0=$ VIVA

* Color negruzco, poco movimiento, mal aspecto en general.

${ }^{1}$ Punto negro.

${ }^{2}$ No se alimenta.

${ }^{3}$ Muda

${ }^{5}$ Aturdida

Ad $=$ Adulto

Tabla 19. Tratamiento Central - 15 segundos

\begin{tabular}{|c|c|c|c|c|c|c|c|c|c|c|c|}
\hline \multirow{2}{*}{ LARVA } & \multirow[b]{2}{*}{$\begin{array}{r}\text { PESO } \\
\text { (mg.) }\end{array}$} & \multicolumn{10}{|c|}{ TIEMPO DE OBSERVACIÓN } \\
\hline & & $\begin{array}{c}\text { 0 } \\
\text { h. }\end{array}$ & $\begin{array}{c}24 \\
h\end{array}$ & $\begin{array}{c}48 \\
h\end{array}$ & $\begin{array}{c}7 \\
\text { días }\end{array}$ & $\begin{array}{c}14 \\
\text { días }\end{array}$ & $\begin{array}{c}21 \\
\text { días }\end{array}$ & $\begin{array}{c}37 \\
\text { días }\end{array}$ & $\begin{array}{c}52 \\
\text { días }\end{array}$ & $\begin{array}{c}\mathbf{6 3} \\
\text { días }\end{array}$ & $\begin{array}{c}70 \\
\text { días }\end{array}$ \\
\hline \multicolumn{12}{|c|}{ Tabla 11 Radial } \\
\hline 1 & 173,6 & 0 & 0 & $0^{1}$ & $0^{1 / 2}$ & $0^{1 / 2}$ & $0^{1 / 2}$ & $0^{1}$ & $0^{3}$ & $0^{3}$ & $0^{2}$ \\
\hline 2 & 173,6 & 0 & 0 & $0^{1}$ & $0^{2}$ & $0^{2}$ & $0^{2}$ & 0 & 0 & 0 & 0 \\
\hline 3 & 186,4 & 1 & - & - & - & - & - & - & - & - & - \\
\hline 4 & 138,1 & 1 & - & - & - & - & - & - & - & - & - \\
\hline 5 & 172,2 & 0 & 0 & $0 *$ & $0 *^{2}$ & $0 *^{2}$ & $0 *^{2}$ & $0 *^{2}$ & $0 * 2 / 3$ & $0^{* 2 / 3}$ & 1 \\
\hline 6 & 172,3 & 0 & 0 & 0 & 0 & 0 & 0 & 0 & 0 & 0 & 0 \\
\hline Total & - & 2 & 2 & 2 & 2 & 2 & 2 & 2 & 2 & 2 & 3 \\
\hline \multicolumn{12}{|c|}{ Tabla 21 Tangencial } \\
\hline 1 & 191,2 & 0 & 0 & 0 & 0 & 0 & 0 & 0 & 0 & 0 & 0 \\
\hline 2 & 195,2 & 0 & 0 & 0 & 0 & 0 & 0 & 0 & 0 & 0 & 0 \\
\hline 3 & 223,2 & 1 & - & - & - & - & - & - & - & - & - \\
\hline 4 & 177,5 & 1 & - & - & - & - & - & - & - & - & - \\
\hline Total & - & 2 & 2 & 2 & 2 & 2 & 2 & 2 & 2 & 2 & 2 \\
\hline
\end{tabular}

Tabla 20. Tratamiento central - 25 segundos

\begin{tabular}{|c|c|c|c|c|c|c|c|c|c|c|c|}
\hline \multirow{2}{*}{ LARVA } & \multirow[b]{2}{*}{$\begin{array}{c}\text { PESO } \\
\text { (mg.) }\end{array}$} & \multicolumn{10}{|c|}{ TIEMPO DE OBSERVACIÓN } \\
\hline & & $\begin{array}{c}\text { 0 } \\
\text { h. }\end{array}$ & $\begin{array}{c}24 \\
\text { h }\end{array}$ & $\begin{array}{c}48 \\
\mathrm{~h}\end{array}$ & $\begin{array}{c}7 \\
\text { días }\end{array}$ & $\begin{array}{c}14 \\
\text { días }\end{array}$ & $\begin{array}{c}21 \\
\text { días }\end{array}$ & $\begin{array}{c}37 \\
\text { días }\end{array}$ & $\begin{array}{c}52 \\
\text { días }\end{array}$ & $\begin{array}{c}63 \\
\text { días }\end{array}$ & $\begin{array}{c}70 \\
\text { días }\end{array}$ \\
\hline \multicolumn{12}{|c|}{ Tabla 32 Tangencial } \\
\hline 1 & 222,8 & $0 *$ & $0^{*}$ & $0^{*}$ & $0^{2}$ & $0 *^{2}$ & $\begin{array}{l}0^{*} \\
2\end{array}$ & $0 *^{2}$ & 1 & - & - \\
\hline 2 & 148,2 & 0 & $0^{*}$ & $0^{*}$ & $0^{2}$ & 0 & 0 & 0 & 0 & 0 & 0 \\
\hline 3 & 207 & 1 & 1 & 1 & 1 & 1 & - & - & - & - & - \\
\hline 4 & 225 & 1 & 1 & 1 & 1 & 1 & - & - & - & - & - \\
\hline 5 & 205 & $0 *$ & $0^{*}$ & $0^{*}$ & $0^{2}$ & $0 *^{2}$ & $\begin{array}{l}0^{*} \\
2\end{array}$ & 1 & - & - & - \\
\hline 6 & 192,7 & 0 & 0 & 0 & $0^{1}$ & 0 & 0 & 0 & 0 & 0 & 0 \\
\hline Total & - & 2 & 2 & 2 & 2 & 2 & 2 & 3 & 4 & 4 & 4 \\
\hline
\end{tabular}


Tabla 21. Tratamiento Central -20 segundos

\begin{tabular}{|c|c|c|c|c|c|c|c|c|c|c|c|}
\hline \multirow[b]{2}{*}{ LARVA } & \multirow[b]{2}{*}{$\begin{array}{c}\text { PESO } \\
\text { (mg.) }\end{array}$} & \multicolumn{10}{|c|}{ TIEMPO DE OBSERVACIÓN } \\
\hline & & $\begin{array}{c}\text { o } \\
\text { h. }\end{array}$ & $\begin{array}{l}24 \\
\text { h }\end{array}$ & $\begin{array}{c}48 \\
\text { h }\end{array}$ & $\begin{array}{c}7 \\
\text { días }\end{array}$ & $\begin{array}{c}14 \\
\text { días }\end{array}$ & $\begin{array}{c}21 \\
\text { días }\end{array}$ & $\begin{array}{c}37 \\
\text { días }\end{array}$ & $\begin{array}{c}52 \\
\text { días }\end{array}$ & $\begin{array}{c}63 \\
\text { días }\end{array}$ & $\begin{array}{c}70 \\
\text { días }\end{array}$ \\
\hline \multicolumn{12}{|c|}{ Tabla 5 Radial } \\
\hline 1 & 188,1 & 0 & 0 & 0 & $0^{2}$ & 0 & 0 & 0 & 0 & 0 & 0 \\
\hline 2 & 186,1 & 0 & $0^{*}$ & $0^{*}$ & $00^{2}$ & 1 & - & - & - & - & - \\
\hline 3 & 191,5 & 1 & - & - & - & - & - & - & - & - & 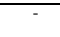 \\
\hline 4 & 185,8 & 1 & - & - & - & - & - & - & - & - & - \\
\hline 5 & 189,5 & $0 *$ & 1 & - & - & - & - & - & - & - & - \\
\hline 6 & 178,2 & 0 & 0 & 0 & 0 & 0 & 0 & 0 & 0 & 0 & 0 \\
\hline Total & - & 2 & 3 & 3 & 3 & 4 & 4 & 4 & 4 & 4 & 4 \\
\hline \multicolumn{12}{|c|}{ Tabla 8 Radial } \\
\hline 1 & 149,3 & 0 & 0 & $0^{1}$ & $0^{2 / 3}$ & 0 & 0 & 0 & 0 & 0 & 0 \\
\hline 2 & 164 & 0 & 0 & $0^{*}$ & 0 & 0 & 0 & 0 & 0 & 0 & 0 \\
\hline 3 & 152,5 & $0 *$ & 0* & $0 *$ & 1 & - & - & - & - & - & - \\
\hline 4 & 170 & 0* & $0 *$ & $0^{*}$ & $0^{*}$ & $0 *^{2}$ & $00^{2}$ & $00^{2}$ & $0 *^{2}$ & 1 & - \\
\hline 5 & 167,7 & 0 & 0 & 0 & $0^{1}$ & 0 & 0 & 0 & 0 & 0 & 0 \\
\hline 6 & 179,7 & 0 & 0 & $0 *$ & $0^{*}$ & $0^{* 2}$ & $0^{*^{2}}$ & $0 *^{2}$ & 1 & - & - \\
\hline Total & - & 0 & 0 & 0 & 1 & 1 & 1 & 1 & 2 & 3 & 3 \\
\hline \multicolumn{12}{|c|}{ Tabla 29 Tangencial } \\
\hline 1 & 189,8 & 0 & 0 & 0 & $0^{2}$ & 0 & 0 & 0 & 0 & $\mathrm{Ad}$ & $\mathrm{Ad}$ \\
\hline 2 & 112,0 & 0 & 0 & $0^{*}$ & $0^{2 / 3}$ & 1 & - & - & - & - & - \\
\hline 3 & 163,5 & 1 & - & - & - & - & - & - & - & - & - \\
\hline 4 & 172,2 & 1 & - & - & - & - & - & - & - & - & - \\
\hline 5 & 197,2 & 0 & 0 & $0^{*}$ & $0^{*}$ & $00^{2}$ & $0 *^{2}$ & $00^{2}$ & 0 *2 $^{2}$ & 1 & - \\
\hline 6 & 157,7 & 0 & 0 & 0 & $0^{2 / 3}$ & 0 & 0 & 0 & 0 & 0 & 0 \\
\hline Total & - & 2 & 2 & 2 & 2 & 3 & 3 & 3 & 3 & 4 & 4 \\
\hline \multicolumn{12}{|c|}{ Tabla 30 Tangencial } \\
\hline 1 & 131,5 & 0 & 0 & 0 & 0 & $0^{2}$ & $0^{2}$ & $0^{2}$ & $0^{3}$ & $0^{3}$ & $0^{3}$ \\
\hline 2 & 132,7 & 0 & 0 & 0 & 0 & 0 & 0 & 0 & 0 & 0 & 0 \\
\hline 3 & 148,1 & 1 & - & - & - & - & - & - & - & - & - \\
\hline 4 & 185,2 & 1 & - & - & - & - & - & - & - & - & - \\
\hline 5 & 153 & 0 & $0^{*}$ & $0^{*}$ & $0^{*}$ & $0^{* 2}$ & $0^{* 2}$ & $0^{* 2}$ & $0^{* 2}$ & $0^{* 2}$ & $0^{* 2}$ \\
\hline 6 & 139,2 & 0 & 0 & 0 & 0 & 0 & 0 & 0 & 0 & 0 & 0 \\
\hline Total & - & 2 & 2 & 2 & 2 & 2 & 2 & 2 & 2 & 2 & 2 \\
\hline
\end{tabular}

Tabla 22. Tratamiento central - Población de control

\begin{tabular}{|c|c|c|c|c|c|c|c|c|c|c|c|}
\hline \multicolumn{10}{|c|}{ Población Control } & \multicolumn{10}{c|}{ TIEMPO DE OBSERVACIÓN } \\
\hline LARVA & $\begin{array}{c}\text { PESO } \\
\text { (mg.) }\end{array}$ & $\begin{array}{c}\mathbf{0} \\
\mathbf{h} .\end{array}$ & $\begin{array}{c}\mathbf{2 4} \\
\mathbf{h}\end{array}$ & $\begin{array}{c}\mathbf{4 8} \\
\mathbf{h}\end{array}$ & $\begin{array}{c}\mathbf{7} \\
\text { días }\end{array}$ & $\begin{array}{c}\mathbf{1 4} \\
\text { días }\end{array}$ & $\begin{array}{c}\mathbf{2 1} \\
\text { días }\end{array}$ & $\begin{array}{c}\mathbf{3 7} \\
\text { días }\end{array}$ & $\begin{array}{c}\mathbf{5 2} \\
\text { días }\end{array}$ & $\begin{array}{c}\mathbf{6 3} \\
\text { días }\end{array}$ & $\begin{array}{c}\mathbf{7 0} \\
\text { días }\end{array}$ \\
\hline 1 & 218 & 0 & 0 & 0 & 0 & 0 & 0 & 0 & 0 & 0 & 0 \\
\hline 2 & 259 & 0 & 0 & 0 & 0 & 0 & 0 & $0^{3}$ & $0^{3}$ & 0 & 0 \\
\hline 3 & 227,9 & 0 & 0 & 0 & 0 & 0 & 0 & 0 & 0 & 0 & 0 \\
\hline 4 & 209,1 & 0 & 0 & 0 & $0^{1}$ & $0^{1}$ & $0^{1}$ & $0^{1}$ & $0^{1}$ & $0^{1 / 2}$ & $0^{1 / 2}$ \\
\hline 5 & 255,9 & 0 & 0 & 0 & 0 & 0 & 0 & 0 & 0 & 0 & 0 \\
\hline 6 & 191,2 & 0 & 0 & 0 & 0 & 0 & 0 & 0 & 0 & 0 & 0 \\
\hline 7 & 220,8 & 0 & 0 & 0 & 0 & 0 & 0 & 0 & 0 & 0 & 0 \\
\hline 8 & 209,8 & 0 & 0 & 0 & 0 & 0 & 0 & 0 & 0 & 0 & 0 \\
\hline 9 & 254 & 0 & 0 & 0 & 0 & 0 & 0 & 0 & 0 & 0 & 0 \\
\hline 10 & 201,3 & 0 & 0 & 0 & 0 & 0 & 0 & 0 & 0 & 0 & 0 \\
\hline 11 & 190,2 & 0 & 0 & 0 & 0 & 0 & 0 & 0 & 0 & 0 & 0 \\
\hline 12 & 203,1 & 0 & 0 & 0 & 0 & 0 & 0 & 0 & 0 & 0 & 0 \\
\hline Total & - & 0 & 0 & 0 & 0 & 0 & 0 & 0 & 0 & 0 & 0 \\
\hline
\end{tabular}


Tabla 23. Tratamiento Solapamiento con tres aplicaciones

\begin{tabular}{|c|c|c|c|c|c|c|c|c|c|c|}
\hline \multirow[b]{2}{*}{ LARVA } & \multirow[b]{2}{*}{$\begin{array}{c}\text { PESO } \\
\text { (mg.) }\end{array}$} & \multicolumn{9}{|c|}{ TIEMPO DE OBSERVACIÓN } \\
\hline & & $0 \mathrm{~h}$. & $24 \mathrm{~h}$ & $\begin{array}{c}7 \\
\text { días }\end{array}$ & $\begin{array}{c}14 \\
\text { días }\end{array}$ & $\begin{array}{c}29 \\
\text { días }\end{array}$ & \begin{tabular}{|c|}
35 \\
días
\end{tabular} & $\begin{array}{c}46 \\
\text { días }\end{array}$ & $\begin{array}{c}53 \\
\text { días }\end{array}$ & $\begin{array}{c}\text { 70 } \\
\text { días }\end{array}$ \\
\hline \multicolumn{11}{|c|}{ Tabla 5 Radial } \\
\hline 1 & 127,73 & 1 & - & - & - & - & - & - & - & - \\
\hline 2 & 170,8 & 1 & - & - & - & - & - & - & - & - \\
\hline 3 & 180,9 & 1 & - & - & - & - & - & - & - & - \\
\hline 4 & 220,3 & 0 & 0 & 0 & 0 & 0 & 0 & 0 & 0 & 0 \\
\hline 5 & 169,8 & 0 & 0 & 0 & 0 & 0 & 0 & 0 & 0 & 0 \\
\hline 6 & 146,1 & 1 & - & - & - & - & - & - & - & - \\
\hline Total & - & 4 & 4 & 4 & 4 & 4 & 4 & 4 & 4 & 4 \\
\hline \multicolumn{11}{|c|}{ Tabla 11 Radial } \\
\hline 1 & 162,8 & 1 & - & - & - & - & - & - & - & - \\
\hline 2 & 8 & 1 & - & - & - & - & - & - & - & - \\
\hline 3 & 207 & 1 & - & - & - & - & - & - & - & - \\
\hline 4 & 238,1 & 0 & $0^{2}$ & $0^{* 2}$ & 0 & 0 & 0 & 0 & 0 & 0 \\
\hline 5 & 137 & 0 & $0^{* 2}$ & $0^{* *^{2}}$ & $0 *^{2}$ & $0^{*^{2}}$ & $0 *^{2}$ & $0^{* 2}$ & $0^{*^{2}}$ & $0^{* 2}$ \\
\hline 6 & 129,8 & 1 & - & - & - & - & - & - & - & - \\
\hline Total & - & 4 & 4 & 4 & 4 & 4 & 4 & 4 & 4 & 4 \\
\hline \multicolumn{11}{|c|}{ Tabla 29 Tangencial } \\
\hline 1 & 95,1 & 1 & - & - & - & - & - & - & - & - \\
\hline 2 & 144 & 1 & - & - & - & - & - & - & - & - \\
\hline 3 & 153,5 & 1 & - & - & - & - & - & - & - & - \\
\hline 4 & 189,1 & 1 & - & - & - & - & - & - & - & - \\
\hline 5 & 151,3 & 1 & - & - & - & - & - & - & - & - \\
\hline 6 & 158,89 & 1 & - & - & - & - & - & - & - & - \\
\hline Total & - & 6 & 6 & 6 & 6 & 6 & 6 & 6 & 6 & 6 \\
\hline \multicolumn{11}{|c|}{ Tabla 30 Tangencial } \\
\hline 1 & 129 & 1 & - & - & - & - & - & - & - & - \\
\hline 2 & 147,9 & 1 & - & - & - & - & - & - & - & - \\
\hline 3 & 125,4 & 1 & - & - & - & - & - & - & - & - \\
\hline 4 & 129,8 & 0 & $0^{2}$ & $0^{2}$ & 0 & 0 & 0 & 0 & 0 & 0 \\
\hline 5 & 133,5 & 1 & - & - & - & - & - & - & - & - \\
\hline 6 & 106,7 & 1 & - & - & - & - & - & - & - & - \\
\hline Total & - & 5 & 5 & 5 & 5 & 5 & 5 & 5 & 5 & 5 \\
\hline
\end{tabular}

Tabla 24. Tratamiento solapamiento con tres aplicaciones- Población de control.

\begin{tabular}{|c|c|c|c|c|c|c|c|c|c|c|}
\hline \multicolumn{1}{|c|}{ Control } & \multicolumn{9}{|c|}{ TIEMPO DE OBSERVACIÓN } \\
\hline LARVA & $\begin{array}{c}\text { PESO } \\
(\mathbf{m g} .)\end{array}$ & $\mathbf{0} \mathbf{~ h .}$ & $\mathbf{2 4} \mathbf{~ h}$ & $\mathbf{7 ~ d}$ & $\mathbf{1 4} \mathbf{d}$ & $\mathbf{2 9} \mathbf{d}$ & $\mathbf{3 5} \mathbf{d}$ & $\mathbf{4 6} \mathbf{d}$ & $\mathbf{5 3 ~ d}$ & $\mathbf{7 0 ~ d}$ \\
\hline 1 & 160,1 & 0 & 0 & 0 & 0 & 0 & 0 & 0 & 0 & 0 \\
\hline 2 & 176,4 & 0 & 0 & 0 & 0 & 0 & 0 & 0 & 0 & 0 \\
\hline 3 & 177,4 & 0 & 0 & 0 & 0 & 0 & 0 & 0 & 0 & 0 \\
\hline 4 & 178,1 & 0 & 0 & 0 & 0 & 0 & 0 & $0^{2}$ & $0^{2}$ & $0^{2}$ \\
\hline 5 & 196,7 & 0 & 0 & 0 & 0 & 0 & 0 & 0 & 0 & 0 \\
\hline 6 & 204,6 & 0 & 0 & 0 & 0 & 0 & 0 & 0 & 0 & 0 \\
\hline 7 & 152,3 & 0 & 0 & 0 & 0 & 0 & 0 & 0 & 0 & 0 \\
\hline 8 & 174,5 & 0 & 0 & $0^{1}$ & $0^{1}$ & $0^{1}$ & 1 & 1 & 1 & 1 \\
\hline 9 & 181,6 & 0 & 0 & 0 & 0 & 0 & 0 & 0 & 0 & 0 \\
\hline 10 & 189,2 & 0 & 0 & 0 & 0 & 0 & 0 & 0 & 0 & 0 \\
\hline 11 & 168,8 & 0 & 0 & 0 & 0 & 0 & 0 & 0 & $0^{*}$ & $0^{*}$ \\
\hline 12 & 186,6 & 0 & 0 & 0 & 0 & 0 & 0 & 0 & 0 & 0 \\
\hline 13 & 170 & 0 & 0 & 0 & 0 & 0 & 0 & 0 & 0 & 0 \\
\hline Total & - & 0 & 0 & 0 & 0 & 0 & 1 & 1 & 1 & 1 \\
\hline
\end{tabular}


Tabla 25. Tratamiento cuatro aplicaciones (50-100mg)

\begin{tabular}{|c|c|c|c|c|c|c|c|}
\hline \multirow{2}{*}{ LARVA } & \multirow{2}{*}{$\begin{array}{c}\text { PESO } \\
(\mathrm{mg})\end{array}$} & \multicolumn{6}{|c|}{ TIEMPO DE OBSERVACIÓN } \\
\hline & & $\mathbf{O} \mathbf{h}$ & $48 \mathrm{~h}$ & 1 sem. & 2 sem. & 3 sem. & 4 sem \\
\hline \multicolumn{8}{|c|}{ Tabla 23 Tangencial } \\
\hline 1 & 92,5 & $0^{5}$ & 0 & 0 & 0 & 0 & 0 \\
\hline 2 & 74,5 & 1 & - & - & - & - & - \\
\hline 3 & 69,9 & 1 & - & - & - & - & - \\
\hline 4 & 72,7 & 1 & - & - & - & - & - \\
\hline 5 & 76,9 & $0^{5}$ & 0 & 0 & 0 & 0 & 0 \\
\hline 6 & 55,2 & 1 & - & - & - & - & - \\
\hline Total & - & 4 & 4 & 4 & 4 & 4 & 4 \\
\hline \multicolumn{8}{|c|}{ Tabla 30 Tangencial } \\
\hline 1 & 84 & $0^{5}$ & 0 & 0 & 0 & 0 & 0 \\
\hline 2 & 77,6 & $0^{5}$ & 0 & 0 & 0 & 0 & 0 \\
\hline 3 & 87,5 & 1 & - & - & - & - & - \\
\hline 4 & 57,2 & 1 & - & - & - & - & - \\
\hline 5 & 60,2 & $0^{5}$ & 0 & 0 & 0 & 0 & 0 \\
\hline 6 & 52 & 1 & - & - & - & - & - \\
\hline Total & - & 3 & 3 & 3 & 3 & 3 & 3 \\
\hline \multicolumn{8}{|c|}{ Tabla 8 Radial } \\
\hline 1 & 81,9 & $0^{5}$ & 0 & 0 & 0 & 0 & 0 \\
\hline 2 & 75,4 & $0^{5}$ & 0 & 0 & 0 & 0 & 0 \\
\hline 3 & 101,3 & 1 & - & - & - & - & - \\
\hline 4 & 72,9 & 1 & - & - & - & - & - \\
\hline 5 & 92,5 & $0^{5}$ & 0 & 0 & 0 & 0 & 0 \\
\hline 6 & 79,4 & 1 & - & - & - & - & - \\
\hline Total & - & 3 & 3 & 3 & 3 & 3 & 3 \\
\hline \multicolumn{8}{|c|}{ Tabla 9 Radial } \\
\hline 1 & 89,9 & 1 & - & - & - & - & - \\
\hline 2 & 84,2 & 1 & - & - & - & - & - \\
\hline 3 & 64,2 & 1 & - & - & - & - & - \\
\hline 4 & 60,1 & 1 & - & - & - & - & - \\
\hline 5 & 74 & $0^{5}$ & 0 & 0 & 0 & 0 & 0 \\
\hline 6 & 96,3 & 1 & - & - & - & - & - \\
\hline Total & - & 5 & 5 & 5 & 5 & 5 & 5 \\
\hline
\end{tabular}

Tabla 26.Tratamiento cuatro aplicaciones (50-100mg)-Población de control

\begin{tabular}{|c|c|c|c|l|l|l|l|}
\hline \multicolumn{2}{|c|}{ Control } & \multicolumn{7}{|c|}{ TIEMPO DE OBSERVACION } \\
\hline LARVA & $\begin{array}{c}\text { PESO } \\
(\mathbf{m g})\end{array}$ & $\mathbf{0} \mathbf{h}$. & $\mathbf{4 8} \mathbf{h}$ & $\mathbf{1}$ sem. & $\mathbf{2}$ sem. & $\mathbf{3}$ sem. & $\mathbf{4}$ sem. \\
\hline 1 & 111,9 & 0 & 0 & 0 & 0 & 0 & 0 \\
\hline 2 & 112,9 & 0 & 0 & 0 & 0 & 0 & 0 \\
\hline 3 & 87,5 & 0 & 0 & 0 & 0 & 0 & 0 \\
\hline 4 & 96 & 0 & 0 & 0 & 0 & 0 & 0 \\
\hline 5 & 82,5 & 0 & 0 & 0 & 0 & 0 & 0 \\
\hline 6 & 103,4 & 0 & 0 & 0 & 0 & 0 & 0 \\
\hline 7 & 90,8 & 0 & 0 & 0 & 0 & 0 & 0 \\
\hline 8 & 104,7 & 0 & 0 & 0 & 0 & 0 & 0 \\
\hline 9 & 83,6 & 0 & 0 & 0 & 0 & 0 & 0 \\
\hline 10 & 94,1 & 0 & 0 & 0 & 0 & 0 & 0 \\
\hline 11 & 106 & 0 & 0 & 0 & 0 & 0 & 0 \\
\hline Total & - & 0 & 0 & 0 & 0 & 0 & 0 \\
\hline
\end{tabular}


Tabla 27. Tratamiento cuatro aplicaciones (100-200mg)

\begin{tabular}{|c|c|c|c|c|c|c|c|}
\hline \multirow{2}{*}{ LARVA } & \multirow{2}{*}{$\begin{array}{c}\text { PESO } \\
(\mathrm{mg})\end{array}$} & \multicolumn{6}{|c|}{ TIEMPO DE OBSERVACIÓN } \\
\hline & & $\mathbf{O} \mathbf{h}$ & $48 \mathrm{~h}$ & 1 sem. & 2 sem. & 3 sem. & 4 sem \\
\hline \multicolumn{8}{|c|}{ Tabla 21 Tangencial } \\
\hline 1 & 116,2 & 1 & - & - & - & - & - \\
\hline 2 & 114,4 & 1 & - & - & - & - & - \\
\hline 3 & 128,8 & 1 & - & - & - & - & - \\
\hline 4 & 150,7 & 1 & - & - & - & - & - \\
\hline 5 & 134,1 & 1 & - & - & - & - & - \\
\hline 6 & 147 & 1 & - & - & - & - & - \\
\hline Total & - & 6 & 6 & 6 & 6 & 6 & 6 \\
\hline \multicolumn{8}{|c|}{ Tabla 29 Tangencial } \\
\hline 1 & 141 & $0^{5}$ & 0 & 0 & 0 & 0 & 0 \\
\hline 2 & 146,1 & $0^{5}$ & 0 & 0 & 0 & 0 & 0 \\
\hline 3 & 119,3 & 1 & - & - & - & - & - \\
\hline 4 & 137,1 & 1 & - & - & - & - & - \\
\hline 5 & 166,3 & 1 & - & - & - & - & - \\
\hline 6 & 202,2 & 1 & - & - & - & - & - \\
\hline Total & - & 4 & 4 & 4 & 4 & 4 & 4 \\
\hline \multicolumn{8}{|c|}{ Tabla 5 Radial } \\
\hline 1 & 155,1 & 0 & 0 & 0 & 0 & 0 & 0 \\
\hline 2 & 112,5 & 0 & 0 & 0 & 0 & 0 & 0 \\
\hline 3 & 167,3 & 1 & - & - & - & - & - \\
\hline 4 & 159,5 & 1 & - & - & - & - & - \\
\hline 5 & 150,4 & 1 & - & - & - & - & - \\
\hline 6 & 159,2 & 1 & - & - & - & - & - \\
\hline Total & - & 4 & 4 & 4 & 4 & 4 & 4 \\
\hline \multicolumn{8}{|c|}{ Tabla 11 Radial } \\
\hline 1 & 159,9 & 0 & 0 & 0 & 0 & 0 & 0 \\
\hline 2 & 127,9 & $0^{5}$ & 0 & 0 & 0 & 0 & 0 \\
\hline 3 & 135 & 1 & - & - & - & - & - \\
\hline 4 & 131,9 & 1 & - & - & - & - & - \\
\hline 5 & 167,4 & 1 & - & - & - & - & - \\
\hline 6 & 150 & 1 & - & - & - & - & - \\
\hline Total & - & 4 & 4 & 4 & 4 & 4 & 4 \\
\hline
\end{tabular}

Tabla 28.Tratamiento cuatro aplicaciones (100-200mg)-Población de control

\begin{tabular}{|c|c|c|c|c|c|c|c|}
\hline \multicolumn{2}{|c|}{ Control } & \multicolumn{6}{|c|}{ TIEMPO DE OBSERVACION } \\
\hline LARVA & $\begin{array}{c}\text { PESO } \\
(\mathbf{m g})\end{array}$ & $\mathbf{0}$ h. & $\mathbf{4 8} \mathbf{h}$ & $\mathbf{1}$ sem. & $\mathbf{2}$ sem. & 3 sem. & 4 sem. \\
\hline 1 & $\mathbf{1 5 4 , 6}$ & 0 & 0 & 0 & 0 & 0 & 0 \\
\hline 2 & 157,9 & 0 & 0 & 0 & 0 & 0 & 0 \\
\hline 3 & 118,2 & 0 & 0 & 0 & 0 & 0 & 0 \\
\hline 4 & 144 & 0 & 0 & 0 & 0 & 0 & 0 \\
\hline 5 & 173,8 & 0 & 0 & 0 & 0 & 0 & 0 \\
\hline 6 & 184,5 & 0 & 0 & 0 & 0 & 0 & 0 \\
\hline 7 & 122,7 & 0 & 0 & 0 & 0 & 0 & 0 \\
\hline 8 & 126,3 & 0 & 0 & 0 & 0 & 0 & 0 \\
\hline 9 & 198,3 & 0 & 0 & 0 & 0 & 0 & 0 \\
\hline 10 & 149,1 & 0 & 0 & 0 & 0 & 0 & 0 \\
\hline Total & - & 0 & 0 & 0 & 0 & 0 & 0 \\
\hline
\end{tabular}

\section{iii. Efecto sobre la estructura y la imprimación}

Las medidas de temperatura tomadas para las muestras tangenciales en los puntos de

la Figura 3-6 se muestran en la Tabla 16. 
Tabla 29. Medidas de Temperatura en prueba de estructura

\begin{tabular}{|c|c|c|c|c|}
\hline \multirow{2}{*}{ Tiempo } & \multicolumn{4}{|c|}{ Temperatura $\left({ }^{\circ} \mathrm{C}\right)$} \\
\hline & Paralelo & Diagonal & Centro & Perpendicular \\
\hline \multicolumn{5}{|c|}{ Tangencial 23} \\
\hline 0 & 12,04 & 11,4 & 13,86 & 13,08 \\
\hline 2,4 & 12,02 & 11,4 & 13,88 & 13,1 \\
\hline 4,8 & 12,04 & 11,4 & 13,98 & 13,16 \\
\hline 7,2 & 12,36 & 11,64 & 15,54 & 13,86 \\
\hline 9,6 & 13,46 & 12,42 & 18,34 & 15,02 \\
\hline 12 & 15,1 & 13,72 & 22,64 & 16,4 \\
\hline 14,4 & 17,04 & 15,02 & 28,08 & 17,7 \\
\hline 16,8 & 19,46 & 16,52 & 34,18 & 19,1 \\
\hline 19,2 & 22,42 & 18,08 & 40,5 & 20,78 \\
\hline 21,6 & 25,36 & 20,02 & 47,08 & 22,6 \\
\hline 24 & 28,36 & 22,06 & 53,56 & - \\
\hline \multicolumn{5}{|c|}{ Tangencial 25} \\
\hline 0 & 12,68 & 11,9 & 14,44 & 13,38 \\
\hline 2,4 & 12,68 & 11,88 & 14,44 & 13,36 \\
\hline 4,8 & 12,68 & 11,88 & 14,5 & 13,46 \\
\hline 7,2 & 12,82 & 12,04 & 14,8 & 13,62 \\
\hline 9,6 & 13 & 12,22 & 15,02 & 13,8 \\
\hline 12 & 13,22 & 12,42 & 15,48 & 14,16 \\
\hline 14,4 & 14 & 13,26 & 16,96 & 15,24 \\
\hline 16,8 & 15,4 & 14,72 & 18,94 & 16,64 \\
\hline 19,2 & 17,16 & 16,38 & 21,68 & 18,14 \\
\hline 21,6 & 19,28 & 18,3 & 25,04 & 19,64 \\
\hline 24 & 21,76 & 20,66 & 28,52 & 21,36 \\
\hline \multicolumn{5}{|c|}{ Tangencial 26} \\
\hline 0 & 14,62 & 13,84 & 16,44 & 15,66 \\
\hline 2,4 & 14,6 & 13,88 & 16,4 & 15,66 \\
\hline 4,8 & 14,6 & 13,92 & 16,68 & 16 \\
\hline 7,2 & 15,08 & 14,52 & 17,78 & 16,82 \\
\hline 9,6 & 16,2 & 15,66 & 19,38 & 17,88 \\
\hline 12 & 17,64 & 17 & 21,6 & 19,18 \\
\hline 14,4 & 19,42 & 18,62 & 24,5 & 20,7 \\
\hline 16,8 & 21,56 & 20,66 & 27,6 & 22,52 \\
\hline 19,2 & 23,8 & 22,92 & 30,92 & 24,38 \\
\hline 21,6 & 26,04 & 25,2 & 34,48 & 26,28 \\
\hline \multicolumn{5}{|c|}{ Tangencial 27} \\
\hline 0 & 14,68 & 13,96 & 16,28 & 15,76 \\
\hline 2,4 & 14,68 & 13,96 & 16,28 & 15,76 \\
\hline 4,8 & 14,68 & 13,94 & 16,46 & 15,92 \\
\hline
\end{tabular}




\begin{tabular}{|c|c|c|c|c|}
\hline 7,2 & 14,92 & 14,32 & 17,76 & 16,58 \\
\hline 9,6 & 15,68 & 15,18 & 19,9 & 17,64 \\
\hline 12 & 16,8 & 16,32 & 23 & 18,9 \\
\hline 14,4 & 18,14 & 17,68 & 26,7 & 20,38 \\
\hline 16,8 & 19,64 & 19,24 & 30,92 & 22,2 \\
\hline 19,2 & 21,26 & 20,92 & 35,46 & 24,02 \\
\hline 21,6 & 22,98 & 22,72 & 40,02 & 25,9 \\
\hline 24 & 24,6 & 24,54 & 44,58 & - \\
\hline \multicolumn{5}{|c|}{ Tangencial 28} \\
\hline 0 & 14,46 & 14 & 16,24 & 15,84 \\
\hline 2,4 & 14,44 & 14,02 & 16,24 & 15,84 \\
\hline 4,8 & 14,44 & 14,04 & 16,24 & 15,84 \\
\hline 7,2 & 14,56 & 14,2 & 17,16 & 16,34 \\
\hline 9,6 & 15,36 & 15,14 & 19,26 & 17,42 \\
\hline 12 & 16,8 & 16,5 & 22,28 & 18,72 \\
\hline 14,4 & 18,56 & 18,16 & 26,44 & 20,38 \\
\hline 16,8 & 20,72 & 20,12 & 31,14 & 22,34 \\
\hline 19,2 & 23,14 & 22,34 & 36,38 & 24,4 \\
\hline 21,6 & 25,5 & 24,58 & 41,6 & 26,58 \\
\hline 24 & 27,9 & 27,02 & 46,94 & 28,8 \\
\hline
\end{tabular}

Las medidas de temperatura sobres las diferentes muestras en la pruebas de imprimación se muestran de la Tabla 17 a la 20 . El tratamiento es de 20 segundos, los datos de tiempo están retrasados aproximadamente 5 segundos, tiempo que requiere el UMI para empezar a transmitir los datos.

Tabla 30. Medidas de temperatura tablas sin imprimación.

\begin{tabular}{|c|c|c|c|c|c|c|}
\hline $\begin{array}{c}\text { TIEMPO } \\
\mathrm{s} \\
\end{array}$ & $\begin{array}{c}\text { Tabla } 1 \\
{ }^{\circ} \mathrm{C} \\
\end{array}$ & $\begin{array}{c}\text { Tabla } 2 \\
{ }^{\circ} \mathrm{C} \\
\end{array}$ & $\begin{array}{c}\text { Tabla } 3 \\
{ }^{\circ} \mathrm{C} \\
\end{array}$ & $\begin{array}{c}\text { Tabla } 4 \\
{ }^{\circ} \mathrm{C} \\
\end{array}$ & $\begin{array}{c}\text { MEDIANA } \\
{ }^{\circ} \mathrm{C} \\
\end{array}$ & DESV. \\
\hline \multicolumn{7}{|c|}{ INTERNA } \\
\hline 0 & 25,46 & 25,22 & 26,1 & 25,18 & 25,34 & 0,3975 \\
\hline 1,8 & 27,56 & 27,36 & 28,76 & 27,56 & 27,56 & 0,6083 \\
\hline 3,6 & 30,22 & 30,22 & 32,06 & 30,58 & 30,4 & 0,8445 \\
\hline 5,4 & 33,26 & 33,6 & 35,92 & 34,12 & 33,86 & 1,0884 \\
\hline 7,2 & 36,54 & 37,34 & 39,96 & 37,98 & 37,66 & 1,2990 \\
\hline 9 & 39,9 & 41,24 & 44,32 & 41,9 & 41,57 & 1,6255 \\
\hline 10,8 & 43,46 & 45,56 & 49,06 & 46,24 & 45,9 & 2,0106 \\
\hline 12,6 & 47,28 & 50,28 & 54,1 & 50,96 & 50,62 & 2,4237 \\
\hline 14,4 & 51,44 & 55,18 & 59 & 55,8 & 55,49 & 2,6886 \\
\hline \multicolumn{7}{|c|}{ RADIACION } \\
\hline 0 & 24,36 & 24,48 & & 24,54 & 24,48 & 0,0775 \\
\hline 1,8 & 25,46 & 25,84 & & 25,96 & 25,84 & 0,2301 \\
\hline
\end{tabular}




\begin{tabular}{|r|r|r|r|r|r|r|}
\hline 3,6 & 26,86 & 27,36 & & 27,7 & 27,36 & 0,3491 \\
\hline 5,4 & 28,42 & 29,18 & & 29,7 & 29,18 & 0,5317 \\
\hline 7,2 & 30,2 & 31,36 & & 31,92 & 31,36 & 0,7437 \\
\hline 9 & 32,1 & 33,54 & & 34,32 & 33,54 & 0,9455 \\
\hline 10,8 & 34,02 & 35,9 & & 36,74 & 35,9 & 1,1888 \\
\hline 12,6 & 36,06 & 38,34 & & 39,22 & 38,34 & 1,4110 \\
\hline 14,4 & 38,12 & 40,78 & & 41,7 & 40,78 & 1,6250 \\
\hline \multicolumn{7}{|c|}{ POSTERIOR } \\
\hline 0 & 23,62 & 23,28 & 25,1 & 24,74 & 24,18 & 0,7556 \\
\hline 1,8 & 24,38 & 23,6 & 26,44 & 25,94 & 25,16 & 1,1499 \\
\hline 3,6 & 25,22 & 24,04 & 27,94 & 27,3 & 26,26 & 1,5743 \\
\hline 5,4 & 26,44 & 24,62 & 29,68 & 28,9 & 27,67 & 2,0229 \\
\hline 7,2 & 27,72 & 25,22 & 31,52 & 30,76 & 29,24 & 2,5485 \\
\hline 9 & 29,02 & 26,04 & 33,56 & 32,88 & 30,95 & 3,0972 \\
\hline 10,8 & 30,34 & 26,92 & 35,78 & 35,36 & 32,85 & 3,7533 \\
\hline 12,6 & 31,46 & 27,76 & 38,18 & 38 & 34,73 & 4,5241 \\
\hline 14,4 & 32,46 & 28,68 & 40,56 & 40,66 & 36,51 & 5,2758 \\
\hline
\end{tabular}

Tabla 31.Medidas de temperatura tablas con imprimación.

\begin{tabular}{|c|c|c|c|}
\hline $\begin{array}{l}\text { TIEMPO } \\
\mathrm{s}\end{array}$ & $\begin{array}{c}\text { INTERIOR } \\
{ }^{\circ} \mathbf{C} \\
\end{array}$ & $\begin{array}{c}\text { RADIACION } \\
{ }^{\circ} \mathrm{C} \\
\end{array}$ & $\begin{array}{c}\text { POSTERIOR } \\
{ }^{\circ} \mathrm{C} \\
\end{array}$ \\
\hline \multicolumn{4}{|c|}{ Tabla 11 - R1 } \\
\hline 0 & 25,44 & 24,38 & 24,42 \\
\hline 1,8 & 27,54 & 25,32 & 25,28 \\
\hline 3,6 & 30,28 & 26,4 & 26,44 \\
\hline 5,4 & 33,52 & 27,72 & 27,76 \\
\hline 7,2 & 37,08 & 29,16 & 29,26 \\
\hline 9 & 40,74 & 30,8 & 30,74 \\
\hline 10,8 & 44,76 & 32,52 & 32,5 \\
\hline 12,6 & 49,1 & 33,98 & 34,54 \\
\hline 14,4 & 53,7 & 35,84 & 36,56 \\
\hline \multicolumn{4}{|c|}{ Tabla 12 - R2 } \\
\hline 0 & 25,32 & 23,54 & 25 \\
\hline 1,8 & 27,56 & 24,24 & 25,88 \\
\hline 3,6 & 30,36 & 25,08 & 26,9 \\
\hline 5,4 & 33,68 & 26,04 & 28,18 \\
\hline 7,2 & 37,34 & 27,12 & 29,56 \\
\hline 9 & 41,1 & 28,42 & 31,14 \\
\hline 10,8 & 45,2 & 29,86 & 32,92 \\
\hline 12,6 & 49,68 & 31,46 & 34,7 \\
\hline 14,4 & 54,46 & 33,04 & 36,56 \\
\hline
\end{tabular}


Tabla 32.Medidas de temperatura tablas con imprimación y tela.

\begin{tabular}{|c|c|c|c|c|c|c|c|c|c|c|}
\hline & \multicolumn{5}{|c|}{ R1 : Aplicación por la madera } & \multicolumn{5}{|c|}{ R2 : Aplicación por la imprimación } \\
\hline & $\begin{array}{l}\mathbf{t} \\
\mathbf{s}\end{array}$ & $\begin{array}{c}\text { Tabla } \\
21 \\
{ }^{\circ} \mathbf{C}\end{array}$ & $\begin{array}{c}\text { Tabla } \\
22 \\
{ }^{\circ} \mathbf{C}\end{array}$ & $\underset{{ }^{\circ} \mathbf{C}}{\text { MED. }}$ & DESV. & $\begin{array}{l}\text { t. } \\
\text { s }\end{array}$ & $\begin{array}{c}\text { Tabla } \\
\mathbf{2 3} \\
{ }^{\circ} \mathbf{C}\end{array}$ & $\begin{array}{c}\text { Tabla } \\
\mathbf{2 4} \\
{ }^{\circ} \mathrm{C}\end{array}$ & $\underset{{ }^{\circ} \mathbf{C}}{\text { MED. }}$ & DESV \\
\hline \multirow{9}{*}{ 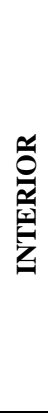 } & 0 & 26,04 & 25 & 25,52 & 0,7354 & 0 & 24,92 & 24,92 & 24,92 & 0,000 \\
\hline & 1,8 & 27,98 & 26,88 &, 43 & 0,7778 & 1,8 & 6,88 & 27,2 & 7,04 & 2263 \\
\hline & 3,6 & & 29,34 & 29,92 & 0,8202 & 3,6 & 29,4 & 30,3 & 29,85 & 0,6364 \\
\hline & 5,4 & 33,38 & 32,28 & 32,83 & 0,7778 & 5,4 & 32,44 & 33,96 & 33,2 & 1,0748 \\
\hline & 7,2 & 36,52 & 35,54 & 36,03 & 0,6930 & 7,2 & 35,72 & 38,04 & 36,88 & 1,6405 \\
\hline & 9 & 39,78 & 38,96 & 39,37 & 0,5798 & 9 & 39,14 & 42,28 & 40,71 & 2,2203 \\
\hline & 10,8 & 43,26 & & 42,88 & 0,5374 & 10,8 & 42,72 & 46,98 & 44,85 & 3,0123 \\
\hline & 12,6 & 46,98 & 46,32 & 46,65 & 0,4667 & 12,6 & 40,34 & 52,18 & 49,36 & 3,9881 \\
\hline & 14,4 & 51,04 & & 50,73 & 0,4384 & 14,4 & & & 54,01 & 4,7093 \\
\hline \multirow{9}{*}{ 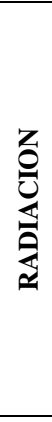 } & & & & & & & & & & \\
\hline & 1,8 & & & & & 1,8 & & & & \\
\hline & 3,6 & & & 7,01 & & 3,6 & & & & 0,7637 \\
\hline & 5,4 & 30,46 & 26,32 & 28,39 & 2,9274 & 5,4 & 2486 & 23,5 & 24,18 & 0,9617 \\
\hline & 7,2 & 32,46 & 27,34 & 29,9 & 3,6204 & 7,2 & 25,3 & 23,68 & 24,49 & 1,1455 \\
\hline & 9 & 34,54 & 28,48 & 31,51 & 4,2851 & 9 & 258 & 2394 & 24,89 & 1,3435 \\
\hline & 10,8 & 36,78 & 29,82 & 33,3 & 4,9215 & 10,8 & 26,38 & 24,22 & 25,3 & 1,5274 \\
\hline & 12,6 & 38,96 & 31 & 5,01 & 5,5861 & 12,6 & 7,08 & 4,4 & 25,74 & 8950 \\
\hline & 14,4 & 41,14 & 32,56 & 6,85 & 0670 & 14,4 & 7,84 & 4,7 & 6,27 & 2,2203 \\
\hline \multirow{9}{*}{ 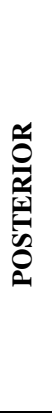 } & 0 & 24,3 & & 24,09 & 0,4101 & $\underline{0}$ & 25,48 & & & 0,4101 \\
\hline & 1,8 & & 24,02 & 24,3 & 0,3960 & 1,8 & & & &, 8344 \\
\hline & 3,6 & 24,74 & & 24,57 & 0,2404 & 3,6 & 27,24 & 25,46 & 26,35 & 1,2587 \\
\hline & 5,4 & 25,02 & 24,84 & 24,93 & 0,1273 & 5,4 & 28,42 & 25,76 & 27,09 & 1,8809 \\
\hline & 7,2 & 25,44 & 25,42 & 25,43 & 0,0141 & 7,2 & 29,04 & 26,14 & 27,89 & 2,4749 \\
\hline & 9 & 25,86 & 26,04 & 25,95 & 0,1273 & 9 & 31,1 & 26,54 & 28,82 & 3,2244 \\
\hline & 10,8 & 26,38 & 26,8 & 26,59 & 0,2970 & 10,8 & 32,62 & 27,06 & 29,84 & 3,9315 \\
\hline & 12,6 & 26,86 & & 27,23 & 0,5233 & 12,6 & 34,42 & 27,7 & 31,06 & 4,7518 \\
\hline & 14,4 & 27,46 & 28,46 & 27,96 & 0,7071 & 14,4 & 36,28 & 28,16 & 32,22 & 5,7417 \\
\hline
\end{tabular}

Tabla 33. Medidas de temperatura tablas con imprimación y estopa.

\begin{tabular}{|r|r|r|r|}
\hline $\begin{array}{c}\text { TIEMPO } \\
\mathbf{S}\end{array}$ & $\begin{array}{c}\text { INTERIOR } \\
{ }^{\mathbf{}} \mathbf{C}\end{array}$ & $\begin{array}{c}\text { RADIACION } \\
{ }^{\mathbf{}} \mathbf{C}\end{array}$ & $\begin{array}{c}\text { OPUESTA } \\
{ }^{\mathbf{}} \mathbf{C}\end{array}$ \\
\hline \multicolumn{4}{|c|}{ Tabla 31 - R1 } \\
\hline 0 & 24,96 & 24,6 & 23,88 \\
\hline 1,8 & 26,74 & 25,66 & 23,94 \\
\hline 3,6 & 29,08 & 26,98 & 24,02 \\
\hline 5,4 & 31,92 & 28,48 & 24,14 \\
\hline 7,2 & 35,08 & 30,24 & 24,24 \\
\hline 9 & 38,48 & 32,16 & 24,44 \\
\hline
\end{tabular}




\begin{tabular}{|r|r|r|r|}
\hline 10,8 & 41,98 & 34,08 & 24,68 \\
\hline 12,6 & 45,82 & 36,22 & 24,94 \\
\hline 14,4 & 49,88 & 38,26 & 25,18 \\
\hline \multicolumn{4}{|c|}{ Tabla 32 - R2 } \\
\hline 0 & 25,04 & 23,5 & 25,62 \\
\hline 1,8 & 27,26 & 23,68 & 27,04 \\
\hline 3,6 & 30,2 & 23,94 & 28,68 \\
\hline 5,4 & 33,64 & 24,26 & 30,56 \\
\hline 7,2 & 37,48 & 24,62 & 32,62 \\
\hline 9 & 41,4 & 25,16 & 34,96 \\
\hline 10,8 & 45,78 & 25,7 & 37,48 \\
\hline 12,6 & 50,48 & 26,4 & 40,14 \\
\hline 14,4 & 55,36 & 27,06 & 42,9 \\
\hline
\end{tabular}

\section{iv. Temperatura adicional}

Las siguientes tablas muestran las medidas de temperatura en listones y nudos.

Tabla 34. Medidas de Temperatura interna en listones

\begin{tabular}{|c|c|c|c|c|c|c|c|c|}
\hline \multirow{2}{*}{$\begin{array}{c}\text { Tiempo } \\
\text { s. }\end{array}$} & \multicolumn{8}{|c|}{ Temperatura $\left({ }^{\circ} \mathrm{C}\right)$} \\
\hline & $\mathbf{L 1}$ & L 2 & L 3 & L4 & $\mathbf{L 5}$ & Mediana & Desviación & Normalizada \\
\hline \multicolumn{9}{|c|}{ Sonda 1 (a $0,5 \mathrm{~cm}$ de la radiación) } \\
\hline 0 & 12,6 & 12,58 & 12,48 & 12,42 & 12,52 & 12,52 & 0,0657 & 0,0052 \\
\hline 2,4 & 13,54 & 13,02 & 13,02 & 12,86 & 14 & 13,02 & 0,5013 & 0,0385 \\
\hline 4,8 & 16,32 & 14,78 & 14,68 & 13,52 & 17,24 & 14,78 & 1,4157 & 0,0958 \\
\hline 7,2 & 21,28 & 17,64 & 17,12 & 14,26 & 22,36 & 17,64 & 3,0732 & 0,1742 \\
\hline 9,6 & 28,68 & 22 & 20,64 & 16,72 & 29,46 & 22 & 5,0991 & 0,2318 \\
\hline 12 & 37,66 & 27,7 & 25,34 & 21,66 & 37,94 & 27,7 & 7,0158 & 0,2533 \\
\hline 14,4 & 48,2 & 34,46 & 30,4 & 29,26 & 47,92 & 34,46 & 9,0938 & 0,2639 \\
\hline 16,8 & 59,06 & 41,9 & 35,96 & 38,48 & 58,26 & 41,9 & 11,0372 & 0,2634 \\
\hline 19,2 & 70,88 & 50,28 & 42,36 & 49,3 & 69,66 & 50,28 & 13,1425 & 0,2614 \\
\hline 21,6 & 83,18 & 58,74 & 49,44 & 60,36 & 81,76 & 60,36 & 14,8363 & 0,2458 \\
\hline 24 & 96,36 & 68,3 & 56,98 & 72,22 & 95,8 & 72,22 & 16,6516 & 0,2306 \\
\hline 26,4 & 99,12 & 80,12 & 75,34 & 83,92 & 98,98 & 83,92 & 10,4489 & 0,1245 \\
\hline 28,8 & 99,68 & 96,62 & 95,96 & 95,82 & 99,5 & 96,62 & 1,9356 & 0,0200 \\
\hline 31,2 & 100,18 & 99,14 & 98,86 & 99,1 & 100,08 & 99,14 & 0,6396 & 0,0065 \\
\hline 33,6 & 101,2 & 99,44 & 99,26 & 99,54 & 101,02 & 99,54 & 1,0034 & 0,0101 \\
\hline 36 & 103,02 & 99,64 & 99,44 & 99,92 & 102,4 & 99,92 & 1,7927 & 0,0179 \\
\hline 38,4 & 106,14 & 99,86 & 99,52 & 100,76 & 104,68 & 100,76 & 3,0548 & 0,0303 \\
\hline 40,8 & 111,14 & 100,24 & 99,68 & 102,24 & 108,94 & 102,24 & 5,1895 & 0,0508 \\
\hline 43,2 & 117,36 & 101,16 & 99,88 & 104,76 & 115,04 & 104,76 & 7,7616 & 0,0741 \\
\hline 45,6 & 123,46 & 103,02 & 100,4 & 109,54 & 121 & 109,54 & 9,4989 & 0,0867 \\
\hline
\end{tabular}




\begin{tabular}{|c|c|c|c|c|c|c|c|c|}
\hline 48 & 128,82 & 106,2 & 101,66 & 115,48 & & 110,84 & 10,6138 & 0,0958 \\
\hline 50,4 & 133,74 & 111,1 & & 120,6 & & 120,6 & 9,3614 & 0,0776 \\
\hline \multicolumn{9}{|c|}{ Sonda 2 (a 1,8 cm de la radiación) } \\
\hline 0 & 11,98 & 11,8 & 11,8 & 11,62 & 11,64 & 11,8 & 0,1345 & 0,0114 \\
\hline 2,4 & 12,62 & 12,48 & 12,72 & 12,08 & 13,1 & 12,62 & 0,3321 & 0,0263 \\
\hline 4,8 & 14,22 & 14,38 & 14,56 & 12,52 & 15,66 & 14,38 & 1,0155 & 0,0706 \\
\hline 7,2 & 16,46 & 16,96 & 16,84 & 13,2 & 18,86 & 16,84 & 1,8702 & 0,1111 \\
\hline 9,6 & 19,38 & 20,56 & 19,9 & 15,18 & 23,56 & 19,9 & 2,6974 & 0,1355 \\
\hline 12 & 23,22 & 25,24 & 23,72 & 18,02 & 29,32 & 23,72 & 3,6445 & 0,1536 \\
\hline 14,4 & 27,68 & 30,56 & 27,98 & 22,34 & 35,56 & 27,98 & 4,3821 & 0,1566 \\
\hline 16,8 & 32,38 & 36,36 & 32,42 & 27,5 & 42,68 & 32,42 & 5,3851 & 0,1661 \\
\hline 19,2 & 37,62 & 42,7 & 37,5 & 33,16 & 50,12 & 37,62 & 6,3555 & 0,1689 \\
\hline 21,6 & 43,2 & 49,2 & 42,88 & 39,68 & 57,88 & 43,2 & 7,2663 & 0,1682 \\
\hline 24 & 48,98 & 55,72 & 48,44 & 46,6 & 66,18 & 48,98 & 8,3333 & 0,1701 \\
\hline 26,4 & 54,82 & 62,74 & 54,06 & 53,38 & 74,54 & 54,82 & 9,5316 & 0,1739 \\
\hline 28,8 & 61,32 & 69,7 & 60,16 & 60,66 & 84,62 & 61,32 & 11,0896 & 0,1808 \\
\hline 31,2 & 68,42 & 76,4 & 66,7 & 67,98 & 95,64 & 68,42 & 12,7103 & 0,1858 \\
\hline 33,6 & 79,14 & 83,4 & 74,88 & 74,98 & 98,58 & 79,14 & 9,2899 & 0,1174 \\
\hline 36 & 94,48 & 91,2 & 89,72 & 82,24 & 99,22 & 91,2 & 5,6134 & 0,0616 \\
\hline 38,4 & 98,18 & 97,3 & 97,08 & 90,5 & 99,62 & 97,3 & 3,2387 & 0,0333 \\
\hline 40,8 & 98,84 & 98,9 & 98,5 & 96,26 & 99,9 & 98,84 & 1,2569 & 0,0127 \\
\hline 43,2 & 99,02 & 99,46 & 98,9 & 98,42 & 100,38 & 99,02 & 0,6954 & 0,0070 \\
\hline 45,6 & 99,2 & 99,88 & 99,12 & 99,24 & 101,26 & 99,24 & 0,9493 & 0,0096 \\
\hline 48 & 99,44 & 100,5 & 99,3 & 99,82 & & 99,63 & 0,4843 & 0,0049 \\
\hline 50,4 & 99,78 & 101,48 & & 100,46 & & 100,46 & 0,7078 & 0,0070 \\
\hline \multicolumn{9}{|c|}{ Sonda 3 (a 3,2 cm de la radiación) } \\
\hline 0 & 13,64 & 13,42 & 13,54 & 13,3 & 13,38 & 13,42 & 0,1255 & 0,0094 \\
\hline 2,4 & 14,84 & 14,38 & 14,84 & 13,74 & 14,9 & 14,84 & 0,5339 & 0,0360 \\
\hline 4,8 & 17,18 & 16,5 & 17,18 & 14,06 & 17,24 & 17,18 & 1,4283 & 0,0831 \\
\hline 7,2 & 20,3 & 19,08 & 20,12 & 14,94 & 19,94 & 19,94 & 2,2760 & 0,1141 \\
\hline 9,6 & 25,2 & 23,16 & 24,62 & 16,88 & 24,2 & 24,2 & 3,3419 & 0,1381 \\
\hline 12 & 30,62 & 27,96 & 29,48 & 19,42 & 28,92 & 28,92 & 4,3445 & 0,1502 \\
\hline 14,4 & 36,64 & 33,24 & 34,6 & 23,48 & 33,96 & 33,96 & 4,8568 & 0,1430 \\
\hline 16,8 & 42,88 & 38,9 & 40,06 & 28,1 & 39,22 & 39,22 & 5,2509 & 0,1339 \\
\hline 19,2 & 49,44 & 44,82 & 45,94 & 33,08 & 44,76 & 44,82 & 5,6645 & 0,1264 \\
\hline 21,6 & 56,04 & 50,84 & 51,94 & 38,44 & 50,34 & 50,84 & 6,0375 & 0,1188 \\
\hline 24 & 62,46 & 56,86 & 57,92 & 43,92 & 56,02 & 56,86 & 6,3346 & 0,1114 \\
\hline 26,4 & 69,02 & 62,62 & 63,76 & 49,56 & 61,54 & 62,62 & 6,5420 & 0,1045 \\
\hline 28,8 & 75,4 & 68,42 & 69,8 & 55,22 & 67,14 & 68,42 & 6,7306 & 0,0984 \\
\hline 31,2 & 81,48 & 74,1 & 75,66 & 60,66 & 72,74 & 74,1 & 6,9193 & 0,0934 \\
\hline 33,6 & 87,54 & 79,54 & 81,38 & 66,04 & 78,42 & 79,54 & 7,0836 & 0,0891 \\
\hline 36 & 94,12 & 84,8 & 87,52 & 71,44 & 84,36 & 84,8 & 7,3884 & 0,0871 \\
\hline
\end{tabular}




\begin{tabular}{|c|c|c|c|c|c|c|c|c|}
\hline 38,4 & 99,1 & 89,96 & 94,5 & 76,58 & 92,1 & 92,1 & 7,7487 & 0,0841 \\
\hline 40,8 & 100,38 & 94,98 & 98,6 & 81,32 & 98,68 & 98,6 & 7,9357 & 0,0805 \\
\hline 43,2 & 100,78 & 98,2 & 100,2 & 86,02 & 100,2 & 100,2 & 6,4095 & 0,0640 \\
\hline 45,6 & 101,24 & 99,72 & 100,96 & 90,58 & & 100,34 & 4,9203 & 0,0490 \\
\hline 48 & 101,88 & 100,62 & 101,64 & 94,9 & & 101,13 & 3,1581 & 0,0312 \\
\hline 50,4 & 102,82 & 101,42 & & 98,1 & & 101,42 & 2,0803 & 0,0205 \\
\hline \multicolumn{9}{|c|}{ Sonda 4 (a 4,2 cm de la radiación) } \\
\hline 0 & 14,36 & 14,08 & 13,68 & 13,96 & 14,44 & 14,08 & 0,2766 & 0,0196 \\
\hline 2,4 & 15,94 & 15,58 & 14,06 & 14,46 & 15,2 & 15,2 & 0,7126 & 0,0469 \\
\hline 4,8 & 18,3 & 18,02 & 15,66 & 14,84 & 17,12 & 17,12 & 1,3808 & 0,0807 \\
\hline 7,2 & 21,46 & 20,98 & 18,08 & 16,04 & 19,46 & 19,46 & 1,9956 & 0,1025 \\
\hline 9,6 & 26,14 & 25,56 & 21,16 & 18,18 & 22,8 & 22,8 & 2,9260 & 0,1283 \\
\hline 12 & 31,62 & 30,98 & 25,98 & 20,86 & 27,3 & 27,3 & 3,8838 & 0,1423 \\
\hline 14,4 & 37,72 & 37,08 & 31,36 & 25,04 & 32,44 & 32,44 & 4,5899 & 0,1415 \\
\hline 16,8 & 43,9 & 43,16 & 37,46 & 29,86 & 37,94 & 37,94 & 5,0653 & 0,1335 \\
\hline 19,2 & 50,62 & 49,72 & 43,66 & 35,26 & 43,52 & 43,66 & 5,5811 & 0,1278 \\
\hline 21,6 & 57,46 & 56,34 & 50,44 & 40,62 & 49,48 & 50,44 & 6,0240 & 0,1194 \\
\hline 24 & 64,42 & 62,8 & 57,4 & 46,2 & 55,74 & 57,4 & 6,4286 & 0,1120 \\
\hline 26,4 & 71,38 & 69,16 & 64,44 & 52,12 & 61,92 & 64,44 & 6,7613 & 0,1049 \\
\hline 28,8 & 78,64 & 75,26 & 71,4 & 57,76 & 68,22 & 71,4 & 7,2592 & 0,1017 \\
\hline 31,2 & 86,94 & 81,6 & 78,48 & 63,46 & 74,5 & 78,48 & 8,0343 & 0,1024 \\
\hline 33,6 & 95,82 & 87,28 & 85,58 & 69,06 & 81,5 & 85,58 & 8,9141 & 0,1042 \\
\hline 36 & 99,4 & 92,68 & 92,28 & 74,5 & 90,2 & 92,28 & 8,6175 & 0,0934 \\
\hline 38,4 & 100,46 & 96,58 & 98,22 & 80,28 & 98,36 & 98,22 & 8,1187 & 0,0827 \\
\hline 40,8 & 101,4 & 98,66 & 99,8 & 86,46 & 99,88 & 99,8 & 6,0303 & 0,0604 \\
\hline 43,2 & 102,52 & 100,36 & 100,36 & 93,48 & 100,2 & 100,36 & 3,2257 & 0,0321 \\
\hline 45,6 & 103,9 & 103,44 & 100,98 & 97,78 & 100,38 & 100,98 & 2,2440 & 0,0222 \\
\hline 48 & 105,74 & 107,28 & 102,24 & 99,1 & & 103,99 & 3,1961 & 0,0307 \\
\hline 50,4 & 108,5 & & 104,66 & 99,78 & & 104,66 & 3,5852 & 0,0343 \\
\hline
\end{tabular}

Tabla 35. Medidas de temperatura superficial en listones

\begin{tabular}{|r|r|r|r|r|r|r|r|r|}
\hline \multirow{2}{*}{$\begin{array}{c}\text { Tiempo } \\
\text { s }\end{array}$} & $\mathbf{1 0}$ Temperatura $\left({ }^{\circ} \mathbf{C}\right)$ \\
\cline { 2 - 9 } & $\mathbf{~ L 7 ~}$ & L8 & L9 & L 10 & Mediana & Desviación & Normalizada \\
\hline \hline \multicolumn{8}{|c|}{ Sonda 1: Lado Superior } \\
\hline 0 & 13,96 & 14,1 & 14,6 & 14,66 & 14,44 & 14,44 & 0,2898 & 0,0201 \\
\hline 2,4 & 14,12 & 14,44 & 14,82 & 14,82 & 14,94 & 14,82 & 0,3602 & 0,0243 \\
\hline 4,8 & 14,26 & 15,8 & 15,9 & 16,02 & 17,16 & 15,9 & 0,9275 & 0,0583 \\
\hline 7,2 & 14,46 & 18,04 & 17,94 & 18,3 & 21,08 & 18,04 & 2,1041 & 0,1166 \\
\hline 9,6 & 15,3 & 22 & 21,12 & 21,7 & 26,84 & 21,7 & 3,6826 & 0,1697 \\
\hline 12 & 17,44 & 25,76 & 24,7 & 25,74 & 32,9 & 25,74 & 4,9242 & 0,1913 \\
\hline 14,4 & 20,98 & 30,74 & 28,9 & 30,98 & 38,8 & 30,74 & 5,7213 & 0,1861 \\
\hline 16,8 & 25,16 & 35,28 & 32,98 & 36,16 & 44,96 & 35,28 & 6,3589 & 0,1802 \\
\hline
\end{tabular}




\begin{tabular}{|c|c|c|c|c|c|c|c|c|}
\hline 19,2 & 30,12 & 39,52 & 37,48 & 40,02 & 51,58 & 39,52 & 6,9024 & 0,1747 \\
\hline 21,6 & 35,58 & 43,54 & 41,94 & 44,84 & 58,56 & 43,54 & 7,6578 & 0,1759 \\
\hline 24 & 40,36 & 46,38 & 47,24 & 50,4 & 65,94 & 47,24 & 9,0305 & 0,1912 \\
\hline 26,4 & 45,86 & 51,04 & 52,86 & 55,84 & 72,8 & 52,86 & 9,5791 & 0,1812 \\
\hline 28,8 & 50 & 55,04 & 59,22 & 62,02 & 78,36 & 59,22 & 9,7638 & 0,1649 \\
\hline 31,2 & 54,76 & 59,16 & 65,06 & 67,52 & 83,76 & 65,06 & 9,9664 & 0,1532 \\
\hline 33,6 & 61,6 & 64,12 & 69,76 & 72,34 & 88,36 & 69,76 & 9,4975 & 0,1361 \\
\hline 36 & 67,88 & 67,96 & 74,12 & 77,04 & 92,78 & 74,12 & 9,3124 & 0,1256 \\
\hline 38,4 & 73,74 & 71,08 & 78,46 & 80,7 & 96,38 & 78,46 & 8,9765 & 0,1144 \\
\hline 40,8 & 78,6 & 74,74 & 82,54 & 83,04 & 99,06 & 82,54 & 8,3609 & 0,1013 \\
\hline 43,2 & 83,6 & 78,12 & 86,58 & 85,58 & 101,76 & 85,58 & 8,0295 & 0,0938 \\
\hline 45,6 & 87,84 & 81 & 90,46 & 87,96 & 104,14 & 87,96 & 7,9561 & 0,0905 \\
\hline 48 & 91,96 & 83,4 & 93,62 & 91,42 & 106,44 & 91,96 & 7,5630 & 0,0822 \\
\hline 50,4 & 95,48 & & & & 107,96 & & & \\
\hline \multicolumn{9}{|c|}{ Sonda 2: Lado izquierdo } \\
\hline 0 & 12,78 & 12,92 & 13,52 & 13,82 & 13,32 & 13,32 & 0,3851 & 0,0289 \\
\hline 2,4 & 12,86 & 13,76 & 13,8 & 14,18 & 13,5 & 13,76 & 0,4595 & 0,0334 \\
\hline 4,8 & 12,96 & 15,24 & 14,36 & 14,84 & 13,98 & 14,36 & 0,7886 & 0,0549 \\
\hline 7,2 & 13,62 & 17,18 & 15,32 & 15,72 & 14,74 & 15,32 & 1,1701 & 0,0764 \\
\hline 9,6 & 14,6 & 19,5 & 16,52 & 16,7 & 15,6 & 16,52 & 1,6399 & 0,0993 \\
\hline 12 & 15,76 & 22,38 & 17,98 & 17,86 & 16,5 & 17,86 & 2,3110 & 0,1294 \\
\hline 14,4 & 17,18 & 25,68 & 19,82 & 19,08 & 17,7 & 19,08 & 3,1503 & 0,1651 \\
\hline 16,8 & 18,72 & 29,1 & 22,04 & 20,42 & 18,82 & 20,42 & 4,0845 & 0,2000 \\
\hline 19,2 & 20,48 & 32,24 & 24,16 & 21,72 & 20,1 & 21,72 & 4,9150 & 0,2263 \\
\hline 21,6 & 22,26 & 35,7 & 26,58 & 23,3 & 21,3 & 23,3 & 5,8241 & 0,2500 \\
\hline 24 & 24,28 & 39,06 & 29,02 & 24,88 & 22,76 & 24,88 & 6,6793 & 0,2685 \\
\hline 26,4 & 26,52 & 42,48 & 31,32 & 26,54 & 23,96 & 26,54 & 7,5311 & 0,2838 \\
\hline 28,8 & 28,72 & 45,94 & 33,78 & 28,06 & 25,36 & 28,72 & 8,1714 & 0,2845 \\
\hline 31,2 & 30,92 & 49,22 & 36,2 & 29,48 & 26,78 & 30,92 & 8,7405 & 0,2827 \\
\hline 33,6 & 33,06 & 52,48 & 38,74 & 31,06 & 28,08 & 33,06 & 9,3616 & 0,2832 \\
\hline 36 & 35,18 & 55,64 & 41,1 & 32,24 & 29,16 & 35,18 & 9,9854 & 0,2838 \\
\hline 38,4 & 37,28 & 58,6 & 43,44 & 33,36 & 30,14 & 37,28 & 10,5720 & 0,2836 \\
\hline 40,8 & 39,18 & 61,7 & 45,42 & 34,64 & 31,1 & 39,18 & 11,2427 & 0,2869 \\
\hline 43,2 & 41,18 & 64,8 & 47,34 & 36,08 & 32,24 & 41,18 & 11,8472 & 0,2877 \\
\hline 45,6 & 43,2 & 67,92 & 48,94 & 37,56 & 33,16 & 43,2 & 12,4630 & 0,2885 \\
\hline 48 & & 70,98 & 50,24 & & 33,82 & 50,24 & 15,2727 & 0,3040 \\
\hline \multicolumn{9}{|c|}{ Sonda 3: Lado Posterior } \\
\hline 0 & 18,58 & 19,46 & 20,92 & 23,04 & 19,88 & 19,88 & 1,6083 & 0,0809 \\
\hline 2,4 & 18,74 & 20 & 21,22 & 23,48 & 20,36 & 20,36 & 1,6265 & 0,0799 \\
\hline 4,8 & 18,92 & 21,08 & 22,06 & 24,3 & 21,52 & 21,52 & 1,7305 & 0,0804 \\
\hline 7,2 & 19,22 & 22,72 & 23,1 & 25,46 & 22,72 & 22,72 & 1,9951 & 0,0878 \\
\hline 9,6 & 19,9 & 24,74 & 24,52 & 27 & 24,16 & 24,52 & 2,3526 & 0,0959 \\
\hline
\end{tabular}




\begin{tabular}{|c|c|c|c|c|c|c|c|c|}
\hline 12 & 21,24 & 27,2 & 26,32 & 28,74 & 25,84 & 26,32 & 2,5561 & 0,0971 \\
\hline 14,4 & 22,94 & 29,76 & 28,18 & 30,7 & 27,64 & 28,18 & 2,7054 & 0,0960 \\
\hline 16,8 & 24,84 & 32,42 & 30,4 & 32,88 & 29,58 & 30,4 & 2,8919 & 0,0951 \\
\hline 19,2 & 27,1 & 35,58 & 32,76 & 35,34 & 31,8 & 32,76 & 3,0843 & 0,0941 \\
\hline 21,6 & 29,4 & 38,74 & 35,42 & 37,84 & 34,32 & 35,42 & 3,2963 & 0,0931 \\
\hline 24 & 31,64 & 42,08 & 38 & 40,38 & 36,7 & 38 & 3,5903 & 0,0945 \\
\hline 26,4 & 34,32 & 45,58 & 40,74 & 43,22 & 39,16 & 40,74 & 3,8285 & 0,0940 \\
\hline 28,8 & 37,04 & 49,26 & 43,76 & 46,06 & 41,82 & 43,76 & 4,1100 & 0,0939 \\
\hline 31,2 & 40,24 & 53,06 & 46,7 & 48,9 & 44,46 & 46,7 & 4,2904 & 0,0919 \\
\hline 33,6 & 43,96 & 56,4 & 49,74 & 51,98 & 47,3 & 49,74 & 4,2127 & 0,0847 \\
\hline 36 & 47,52 & 60,06 & 52,72 & 55,2 & 50,06 & 52,72 & 4,3392 & 0,0823 \\
\hline 38,4 & 50,38 & 63,78 & 55,82 & 57,92 & 52,78 & 55,82 & 4,6175 & 0,0827 \\
\hline 40,8 & 52,98 & 67,52 & 58,68 & 60,64 & 55,58 & 58,68 & 4,9817 & 0,0849 \\
\hline 43,2 & 55,98 & 71,28 & 61,72 & 63,62 & 58,3 & 61,72 & 5,2848 & 0,0856 \\
\hline 45,6 & 58,5 & 74,98 & 64,56 & 66,9 & 61,34 & 64,56 & 5,6770 & 0,0879 \\
\hline 48 & 60,94 & 78,42 & 67,9 & 70,38 & 64,44 & 67,9 & 5,9537 & 0,0877 \\
\hline \multicolumn{9}{|c|}{ Sonda 4: Lado Derecho } \\
\hline 0 & 14,96 & 14,56 & 15,26 & 15,54 & 15,94 & 15,26 & 0,4735 & 0,0310 \\
\hline 2,4 & 15,04 & 14,88 & 15,6 & 15,86 & 16,2 & 15,6 & 0,5019 & 0,0322 \\
\hline 4,8 & 15,26 & 15,4 & 16,18 & 16,52 & 16,66 & 16,18 & 0,6001 & 0,0371 \\
\hline 7,2 & 15,76 & 16,02 & 16,96 & 17,38 & 17,28 & 16,96 & 0,7214 & 0,0425 \\
\hline 9,6 & 16,58 & 16,66 & 17,72 & 18,34 & 18,04 & 17,72 & 0,7629 & 0,0431 \\
\hline 12 & 17,48 & 17,4 & 18,56 & 19,36 & 18,9 & 18,56 & 0,8084 & 0,0436 \\
\hline 14,4 & 18,44 & 18,14 & 19,48 & 20,4 & 19,76 & 19,48 & 0,8720 & 0,0448 \\
\hline 16,8 & 19,36 & 18,94 & 20,32 & 21,66 & 20,66 & 20,32 & 0,9734 & 0,0479 \\
\hline 19,2 & 20,36 & 19,68 & 21,28 & 23 & 21,56 & 21,28 & 1,1352 & 0,0533 \\
\hline 21,6 & 21,6 & 20,44 & 22,44 & 24,48 & 22,5 & 22,44 & 1,3320 & 0,0594 \\
\hline 24 & 23,06 & 21,3 & 23,58 & 26,02 & 23,5 & 23,5 & 1,5093 & 0,0642 \\
\hline 26,4 & 24,64 & 22,3 & 24,9 & 27,38 & 24,68 & 24,68 & 1,6127 & 0,0653 \\
\hline 28,8 & 26,12 & 23,46 & 26,18 & 28,78 & 26,06 & 26,12 & 1,6828 & 0,0644 \\
\hline 31,2 & 27,54 & 24,64 & 27,62 & 30,34 & 27,42 & 27,54 & 1,8039 & 0,0655 \\
\hline 33,6 & 29,12 & 25,6 & 29,04 & 32,06 & 28,56 & 29,04 & 2,0587 & 0,0709 \\
\hline 36 & 30,52 & 26,6 & 30,44 & 33,68 & 29,88 & 30,44 & 2,2611 & 0,0743 \\
\hline 38,4 & 32,06 & 27,72 & 31,68 & 35,28 & 31,14 & 31,68 & 2,4115 & 0,0761 \\
\hline 40,8 & 33,64 & 28,88 & 33,14 & 36,72 & 32,56 & 33,14 & 2,5120 & 0,0758 \\
\hline 43,2 & 35,18 & 30,26 & 34,62 & 38,14 & 33,88 & 34,62 & 2,5401 & 0,0734 \\
\hline 45,6 & 36,66 & 31,54 & 35,96 & 39,68 & 35,32 & 35,96 & 2,6182 & 0,0728 \\
\hline 48 & & 32,9 & 37,36 & 41,24 & 36,7 & 37,03 & 2,9580 & 0,0799 \\
\hline
\end{tabular}


Tabla 36. Medidas de temperatura en nudos

\begin{tabular}{|c|c|c|c|c|c|}
\hline \multirow{2}{*}{$\begin{array}{c}\text { Tiempo } \\
\text { s }\end{array}$} & \multicolumn{5}{|c|}{ Temperatura $\left({ }^{\circ} \mathrm{C}\right)$} \\
\hline & T 12 & T 13 & Mediana & Desviación & Normalizada \\
\hline \multicolumn{6}{|c|}{ Sonda 1: Nudo debajo } \\
\hline 0 & 17,34 & 15,5 & 16,42 & 0,9200 & 0,056029233 \\
\hline 1,2 & 17,36 & 15,5 & 16,43 & 0,9300 & 0,056603774 \\
\hline 2,4 & 17,34 & 15,5 & 16,42 & 0,9200 & 0,056029233 \\
\hline 3,6 & 17,34 & 15,48 & 16,41 & 0,9300 & 0,056672761 \\
\hline 4,8 & 17,34 & 15,5 & 16,42 & 0,9200 & 0,056029233 \\
\hline 6 & 17,34 & 15,52 & 16,43 & 0,9100 & 0,055386488 \\
\hline 7,2 & 17,4 & 15,6 & 16,5 & 0,9000 & 0,054545455 \\
\hline 8,4 & 17,56 & 15,7 & 16,63 & 0,9300 & 0,055923031 \\
\hline 9,6 & 17,84 & 15,9 & 16,87 & 0,9700 & 0,057498518 \\
\hline 10,8 & 18,2 & 16,14 & 17,17 & 1,0300 & 0,059988352 \\
\hline 12 & 18,74 & 16,48 & 17,61 & 1,1300 & 0,064168086 \\
\hline 13,2 & 19,3 & 16,86 & 18,08 & 1,2200 & 0,067477876 \\
\hline 14,4 & 19,92 & 17,32 & 18,62 & 1,3000 & 0,069817401 \\
\hline 15,6 & 20,64 & 17,84 & 19,24 & 1,4000 & 0,072765073 \\
\hline 16,8 & 21,38 & 18,36 & 19,87 & 1,5100 & 0,075993961 \\
\hline 18 & 22,18 & 18,88 & 20,53 & 1,6500 & 0,08037019 \\
\hline 19,2 & 22,86 & 19,38 & 21,12 & 1,7400 & 0,082386364 \\
\hline 20,4 & 23,46 & 19,86 & 21,66 & 1,8000 & 0,083102493 \\
\hline 21,6 & 24,1 & 20,34 & 22,22 & 1,8800 & 0,084608461 \\
\hline 22,8 & 24,52 & 20,74 & 22,63 & 1,8900 & 0,083517455 \\
\hline 24 & 25,02 & 21,16 & 23,09 & 1,9300 & 0,083585968 \\
\hline 25,2 & 25,36 & & 25,36 & & \\
\hline \multicolumn{6}{|c|}{ Sonda 2: Superior centro } \\
\hline 0 & 16,36 & 15,5 & 15,93 & 0,4300 & 0,026993095 \\
\hline 1,2 & 16,38 & 15,5 & 15,94 & 0,4400 & 0,027603513 \\
\hline 2,4 & 16,38 & 15,5 & 15,94 & 0,4400 & 0,027603513 \\
\hline 3,6 & 16,38 & 15,48 & 15,93 & 0,4500 & 0,028248588 \\
\hline 4,8 & 16,34 & 15,5 & 15,92 & 0,4200 & 0,02638191 \\
\hline 6 & 16,4 & 15,52 & 15,96 & 0,4400 & 0,027568922 \\
\hline 7,2 & 16,56 & 15,6 & 16,08 & 0,4800 & 0,029850746 \\
\hline 8,4 & 16,88 & 15,7 & 16,29 & 0,5900 & 0,036218539 \\
\hline 9,6 & 17,3 & 15,9 & 16,6 & 0,7000 & 0,042168675 \\
\hline 10,8 & 17,8 & 16,14 & 16,97 & 0,8300 & 0,048909841 \\
\hline 12 & 18,4 & 16,48 & 17,44 & 0,9600 & 0,055045872 \\
\hline 13,2 & 19,14 & 16,86 & 18 & 1,1400 & 0,063333333 \\
\hline 14,4 & 20 & 17,32 & 18,66 & 1,3400 & 0,071811361 \\
\hline 15,6 & 20,84 & 17,84 & 19,34 & 1,5000 & 0,077559462 \\
\hline
\end{tabular}




\begin{tabular}{|r|r|r|r|r|r|}
16,8 & 21,9 & 18,36 & 20,13 & 1,7700 & 0,087928465 \\
\hline 18 & 22,92 & 18,88 & 20,9 & 2,0200 & 0,096650718 \\
\hline 19,2 & 23,94 & 19,38 & 21,66 & 2,2800 & 0,105263158 \\
\hline 20,4 & 25,1 & 19,86 & 22,48 & 2,6200 & 0,116548043 \\
\hline 21,6 & 26,32 & 20,34 & 23,33 & 2,9900 & 0,128161166 \\
\hline 22,8 & 27,5 & 20,74 & 24,12 & 3,3800 & 0,14013267 \\
\hline 24 & 28,6 & 21,16 & 24,88 & 3,7200 & 0,149517685 \\
\hline
\end{tabular}




\section{APÉNDICE III. PLANOS}

Plano 1. Modelo (a) - Detalle del modelo cuadrado utilizado en el método de desinsectación de madera para radiaciones solapadas.
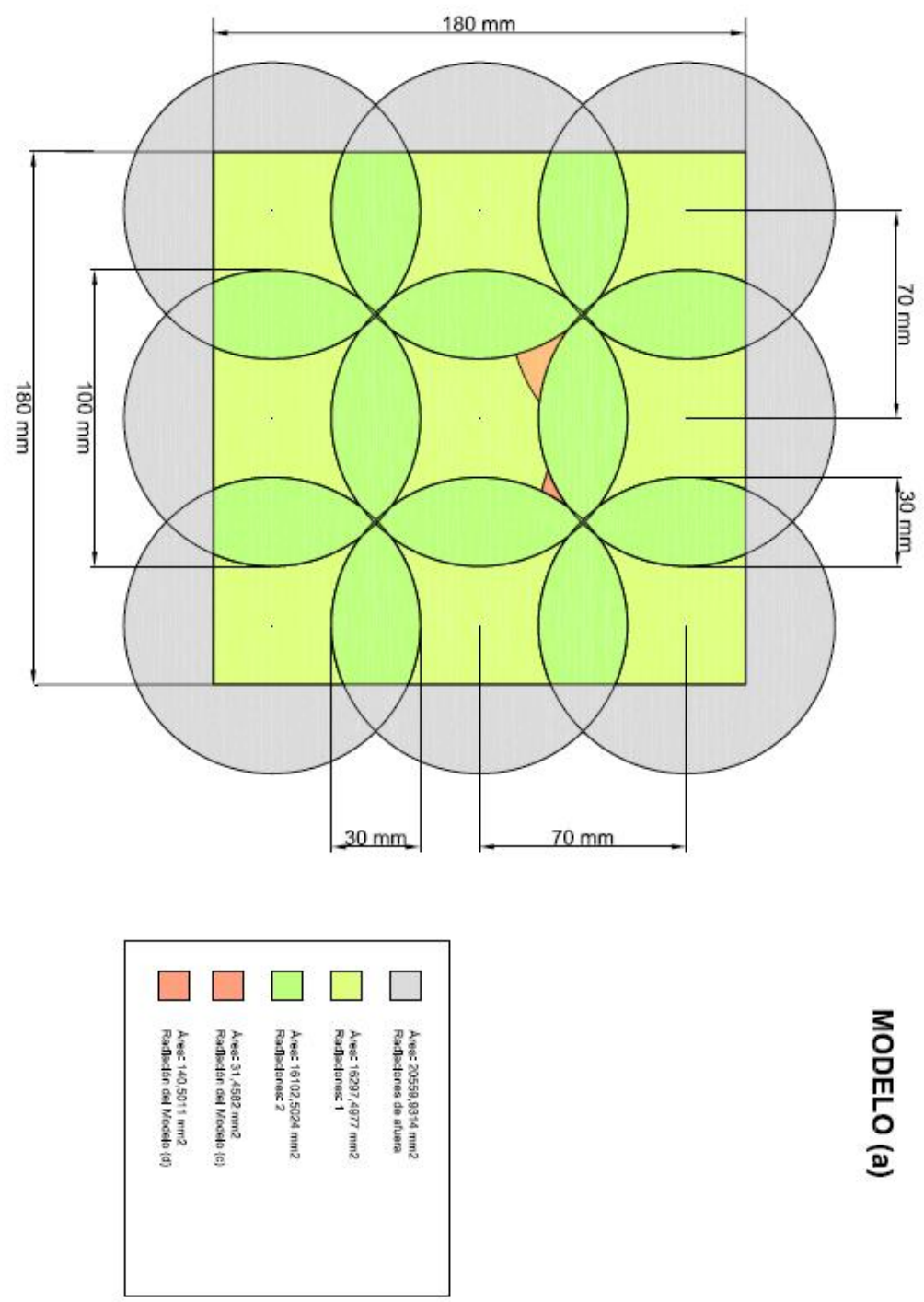
Plano 2. Modelo (b) - Detalle del segundo modelo cuadrado propuesto en el método de desinsectación de madera para radicaciones solapadas.
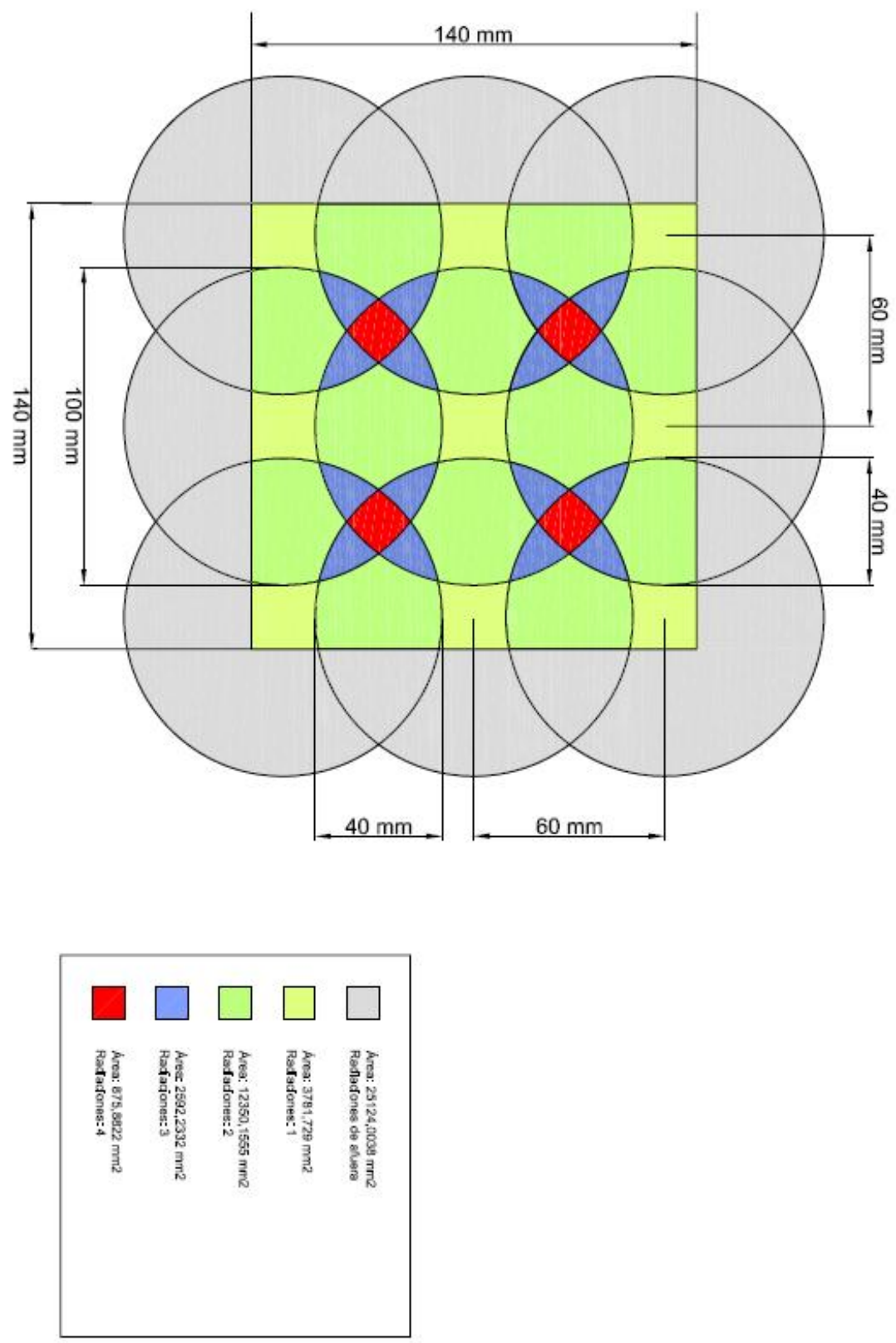

3
음
뜨
옹 
Plano 3. Modelo (c) - Detalle del modelo triangular propuesto en el método de desinsectación de madera para radiaciones solapadas.
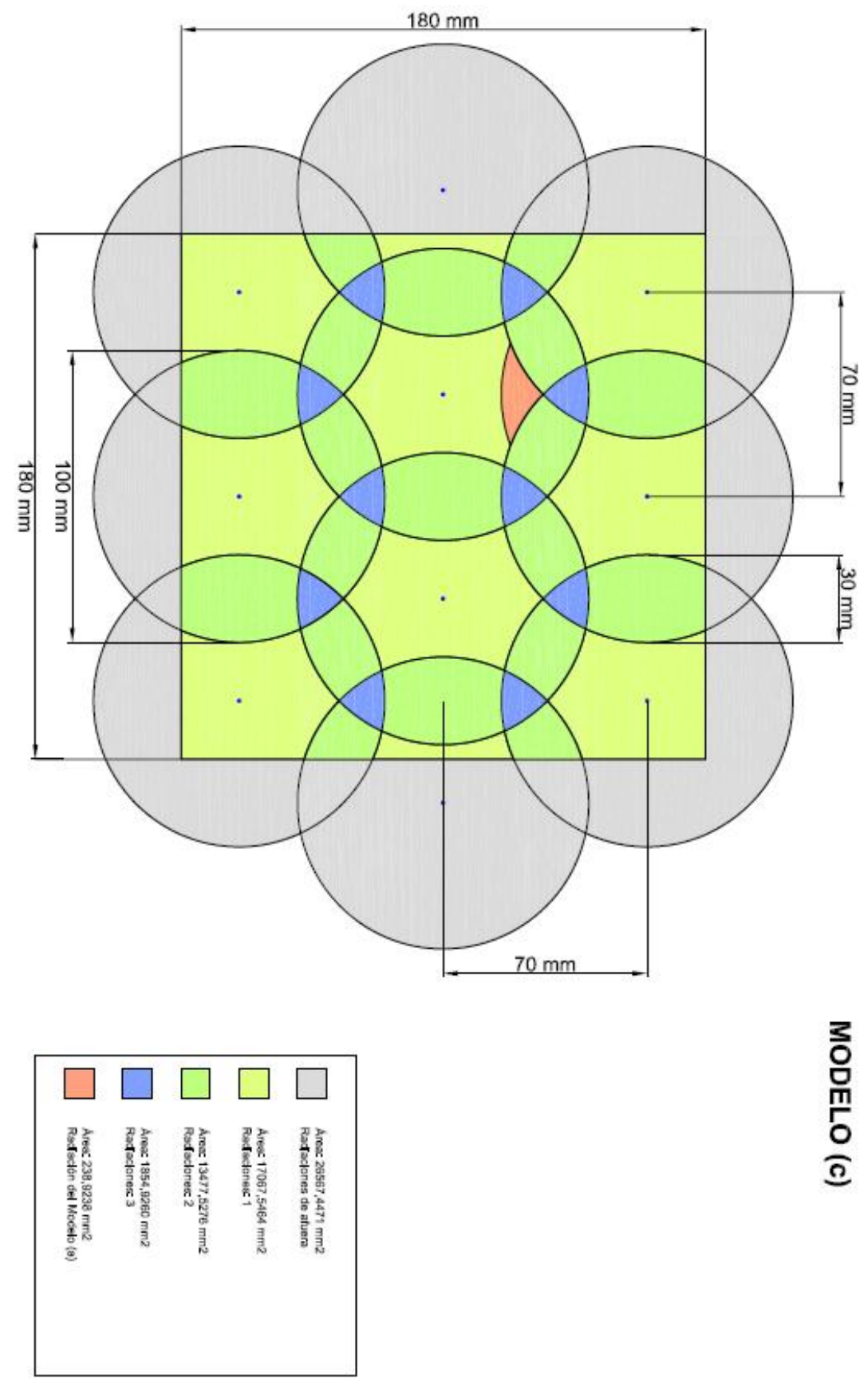

3
음
m
을 
Plano 4. Modelo (d) - Detalle del modelo triangular equilátero propuesto en el método de desinsectación de madera para radiaciones solapadas.
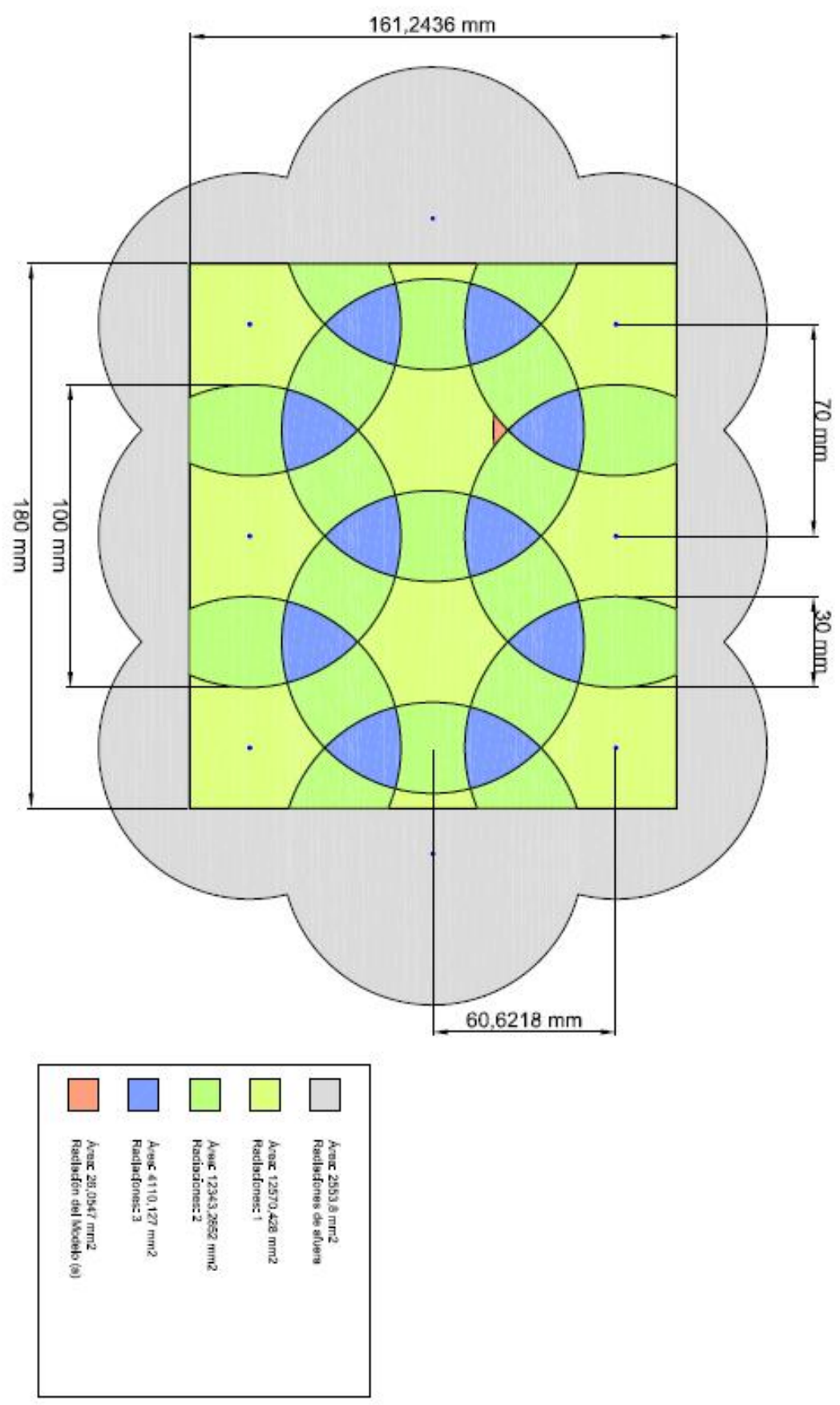

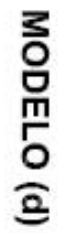




\section{APÉNDICE IV. CAMPO ELÉCTRICO DEL DISEÑO BÁSICO}

En las siguientes tablas se encuentran la magnitud y fase del campo eléctrico del diseño básico utilizado.

Estas grafías están organizadas por modo de funcionamiento, primero el modo A (2 $\mathrm{kW})$ y luego el B $(1 \mathrm{~kW})$. Dentro de ésta división se tienen diferentes planos de observación el primero es el plano de apertura del aplicador, el segundo esta $2 \mathrm{~cm}$ de dicho plano y los cuatro siguientes distanciados $\lambda \mathrm{o} / 10$. 


\begin{tabular}{|c|c|c|c|c|}
\hline Modo A & Apertura & $\mathbf{E x}$ & Ey & Relación Axial (lineal) y Desfase \\
\hline Mag & 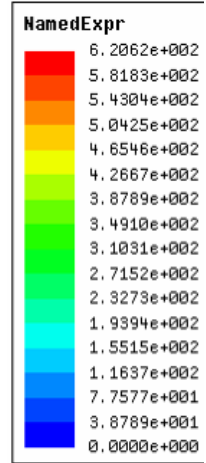 & & & 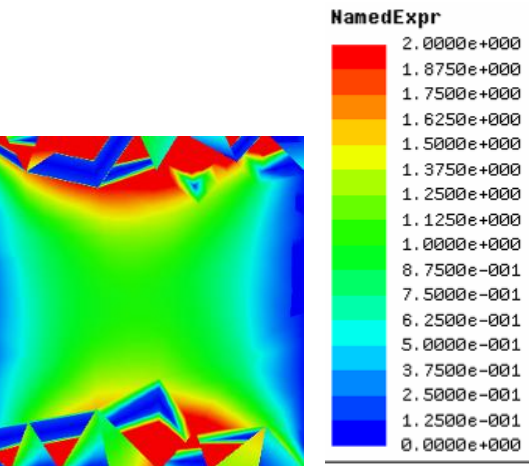 \\
\hline Fase & 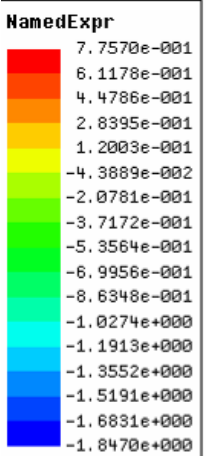 & & & 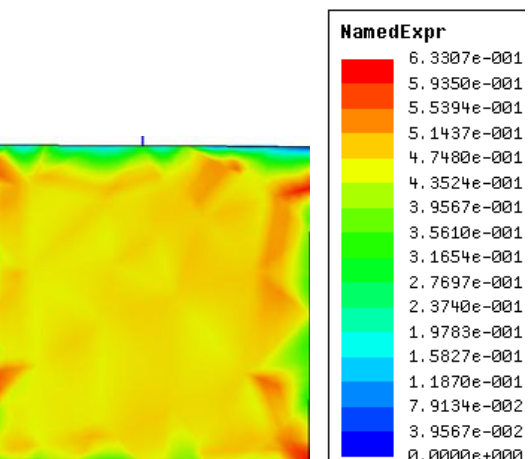 \\
\hline
\end{tabular}




\begin{tabular}{|c|c|c|c|c|}
\hline Modo A & Plano 1 - 2 cm & $\mathbf{E x}$ & Ey & Relación Axial (lineal) y Desfase \\
\hline Mag & 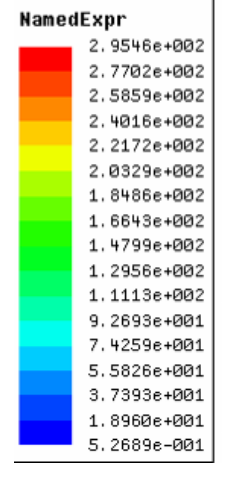 & & & 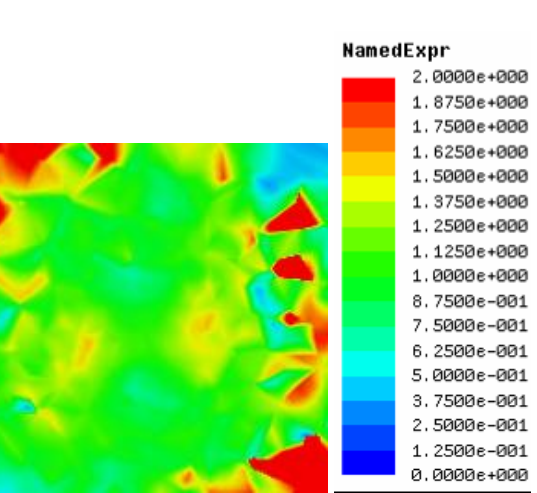 \\
\hline Fase & 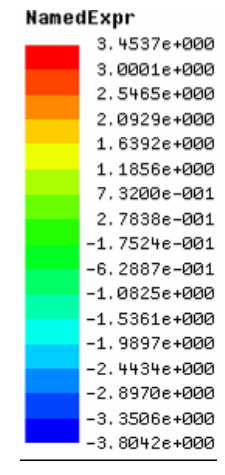 & & & 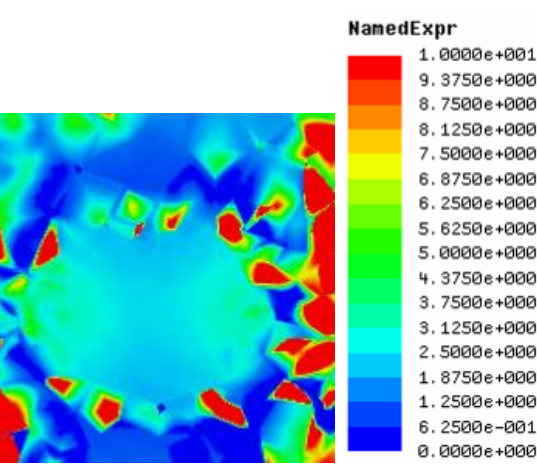 \\
\hline
\end{tabular}




\begin{tabular}{|c|c|c|c|c|}
\hline Modo A & Plano 2 & $\mathbf{E x}$ & Ey & Relación Axial (lineal) y Desfase \\
\hline Mag & 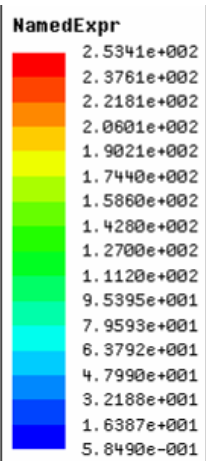 & & & 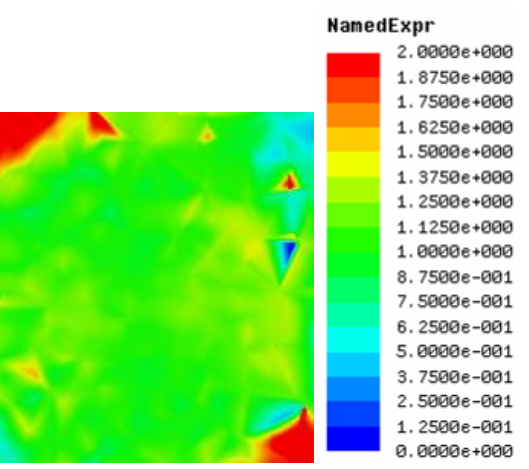 \\
\hline Fase & $\begin{array}{l}\text { NamedExpr } \\
\begin{array}{|l|}3.5387 e+000 \\
3.0902 e+000 \\
2.6417 e+000 \\
2.1932 e+000 \\
1.7447 e+000 \\
1.2962 e+000 \\
8.4776 e-001 \\
3.9926 e-001 \\
-4.9227 e-002 \\
-4.9772 e-001 \\
-9.4621 e-001 \\
-1.3947 e+000 \\
-1.8432 e+000 \\
-2.2917 e+000 \\
-2.7402 e+000 \\
-3.1887 e+000 \\
-3.6372 e+000\end{array}\end{array}$ & & & \begin{tabular}{|c|} 
NamedExpr \\
$1.0000 e+001$ \\
$9.3750 e+000$ \\
$8.7500 e+000$ \\
$8.1250 e+000$ \\
$7.5000 e+000$ \\
$6.8750 e+000$ \\
$6.2500 e+000$ \\
$5.6250 e+000$ \\
$5.0000 e+000$ \\
$4.3750 e+000$ \\
$3.7500 e+000$ \\
$3.1250 e+000$ \\
$2.5000 e+000$ \\
$1.8750 e+000$ \\
$1.2500 e+000$ \\
$6.2500 e-001$ \\
$0.0000 e+000$
\end{tabular} \\
\hline
\end{tabular}




\begin{tabular}{|c|c|c|c|c|}
\hline Modo A & Plano 3 & $\mathbf{E x}$ & Ey & Relación Axial (lineal) y Desfase \\
\hline Mag & 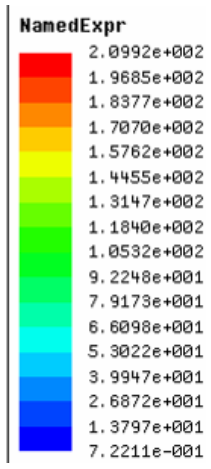 & & & \begin{tabular}{|c|c} 
NamedExpr \\
$2.0000 \mathrm{e}+000$ \\
$1.8750 \mathrm{e}+000$ \\
$1.7500 \mathrm{e}+000$ \\
$1.6250 \mathrm{e}+000$ \\
$1.5000 \mathrm{e}+000$ \\
$1.3750 \mathrm{e}+000$ \\
$1.2500 \mathrm{e}+000$ \\
$1.1250 \mathrm{e}+000$ \\
$1.0000 \mathrm{e}+000$ \\
$8.7500 \mathrm{e}-001$ \\
$7.5000 \mathrm{e}-001$ \\
$6.2500 \mathrm{e}-001$ \\
$5.0000 \mathrm{e}-001$ \\
$3.7500 \mathrm{e}-001$ \\
$2.5000 \mathrm{e}-001$ \\
$1.2500 \mathrm{e}-001$ \\
$0.0000 \mathrm{e}+000$
\end{tabular} \\
\hline Fase & \begin{tabular}{|} 
NamedExpr \\
$3.6543 e+000$ \\
$3.1978 e+000$ \\
$2.7413 e+000$ \\
$2.2849 e+000$ \\
$1.8284 e+000$ \\
$1.3720 e+000$ \\
$9.1550 e-001$ \\
$4.5904 e-001$ \\
$2.5847 e-003$ \\
$-4.5387 e-001$ \\
$-9.1033 e-001$ \\
$-1.3668 e+000$ \\
$-1.8232 e+000$ \\
$-2.2797 e+000$ \\
$-2.7362 e+000$ \\
$-3.1926 e+000$ \\
$-3.6491 e+000$
\end{tabular} & & & \begin{tabular}{|c|} 
NamedExpr \\
$1.0000 e+001$ \\
$9.3750 e+\emptyset 00$ \\
$8.7500 e+000$ \\
$8.1250 e+000$ \\
$7.5000 e+000$ \\
$6.8750 e+000$ \\
$6.2500 e+000$ \\
$5.6250 e+000$ \\
$5.0000 e+000$ \\
$4.3750 e+000$ \\
$3.7500 e+000$ \\
$3.1250 e+000$ \\
$2.5000 e+000$ \\
$1.8750 e+000$ \\
$1.2500 e+000$ \\
$6.2500 e-001$ \\
$0.0000 e+000$
\end{tabular} \\
\hline
\end{tabular}




\begin{tabular}{|c|c|c|c|c|}
\hline Modo A & Plano 4 & $\mathbf{E x}$ & Ey & Relación Axial (lineal) y Desfase \\
\hline Mag & 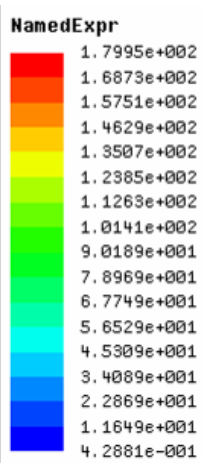 & & & \begin{tabular}{|c|} 
NamedExpr \\
$2.0000 e+000$ \\
$1.8750 e+000$ \\
$1.7500 e+000$ \\
$1.6250 e+000$ \\
$1.5000 e+000$ \\
$1.3750 e+000$ \\
$1.2500 e+000$ \\
$1.1250 e+000$ \\
$1.0000 e+000$ \\
$8.7500 e-001$ \\
$7.5000 e-001$ \\
$6.2500 e-001$ \\
$5.0000 e-001$ \\
$3.7500 e-001$ \\
$2.5000 e-001$ \\
$1.2500 e-001$ \\
$0.0000 e+000$
\end{tabular} \\
\hline Fase & $\begin{array}{l}\text { NamedExpr } \\
\begin{array}{r}3.7127 e+000 \\
3.2527 e+000 \\
2.7927 e+000 \\
2.3326 e+000 \\
1.8726 e+000 \\
1.4126 e+000 \\
9.5253 e-001 \\
4.9250 e-001 \\
3.2462 e-002 \\
-4.2757 e-001 \\
-8.8760 e-001 \\
-1.3476 e+000 \\
-1.8077 e+000 \\
-2.2677 e+000 \\
-2.7277 e+000 \\
-3.1878++000 \\
-3.6478 e+000\end{array}\end{array}$ & & & \begin{tabular}{|c|c} 
NamedExpr \\
$1.0000 e+001$ \\
$9.3750 e+000$ \\
$8.7500 e+000$ \\
$8.1250 e+000$ \\
$7.5000 e+000$ \\
$6.8750 e+000$ \\
$6.2500 e+000$ \\
$5.6250 e+000$ \\
$5.0000 e+000$ \\
$4.3750 e+000$ \\
$3.7500 e+000$ \\
$3.1250 e+000$ \\
$2.5000 e+000$ \\
$1.8750 e+000$ \\
$1.2500 e+000$ \\
$6.2500 e-001$ \\
$0.0000 e+000$
\end{tabular} \\
\hline
\end{tabular}




\begin{tabular}{|c|c|c|c|c|}
\hline Modo A & Plano 5 & $\mathbf{E x}$ & Ey & Relación Axial (lineal) y Desfase \\
\hline Mag & $\begin{array}{l}\text { NamedExpr } \\
\begin{aligned} 1.6061 e+002 \\
1.5061 e+002 \\
1.4061 e+002 \\
1.3061 e+002 \\
1.2060 e+002 \\
1.1060 e+002 \\
1.0060 e+002 \\
9.0597 e+001 \\
8.0594 e+001 \\
7.0592 e+001 \\
6.0590 e+001 \\
5.0587 e+001 \\
4.0585 e+001 \\
3.0583 e+001 \\
2.0581 e+001 \\
1.0578 e+001 \\
5.7596 e-001\end{aligned}\end{array}$ & & & \begin{tabular}{|l|l} 
NamedExpr \\
$2.0000 \mathrm{e}+000$ \\
$1.8750 \mathrm{\theta}+000$ \\
$1.7500 \mathrm{e}+000$ \\
$1.6250 \mathrm{e}+000$ \\
$1.5000 \mathrm{e}+000$ \\
$1.3750 \mathrm{e}+000$ \\
$1.2500 \mathrm{e}+000$ \\
$1.1250 \mathrm{e}+000$ \\
$1.0000 \mathrm{e}+000$ \\
$8.7500 \mathrm{e}-001$ \\
$7.5000 \mathrm{e}-001$ \\
$6.2500 \mathrm{e}-001$ \\
$5.0000 \mathrm{e}-001$ \\
$3.7500 \mathrm{e}-001$ \\
$2.5000 \mathrm{e}-001$ \\
$1.2500 \mathrm{e}-001$ \\
$0.0000 \mathrm{e}+000$
\end{tabular} \\
\hline Fase & $\begin{array}{l}\text { NamedExpr } \\
\begin{array}{r}3.5557 e+000 \\
3.1143 e+000 \\
2.6730 e+000 \\
2.2316 e+000 \\
1.7903 e+000 \\
1.3489 e+000 \\
9.0760 e-001 \\
4.6625 e-001 \\
2.4898 e-002 \\
-4.1645 e-001 \\
-8.5780 e-001 \\
-1.2992 e+000 \\
-1.7405 e+000 \\
-2.1818 e+000 \\
-2.6232 e+000 \\
-3.0645 e+000 \\
-3.5059 e+000\end{array}\end{array}$ & & & 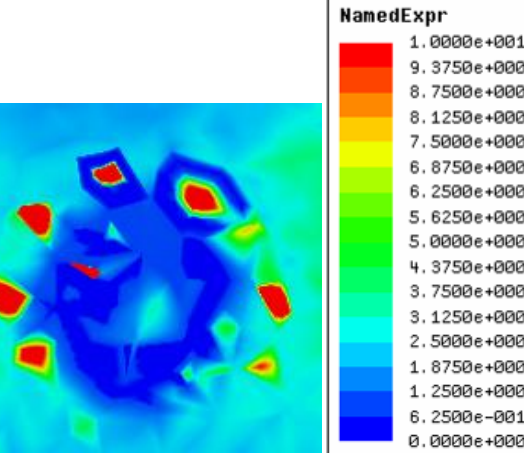 \\
\hline
\end{tabular}




\begin{tabular}{|c|c|c|c|c|}
\hline Modo B & Apertura & $\mathbf{E x}$ & Ey & Relación Axial (lineal) y Desfase \\
\hline Mag & 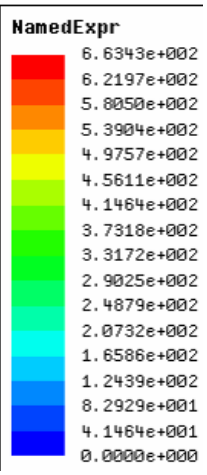 & & & 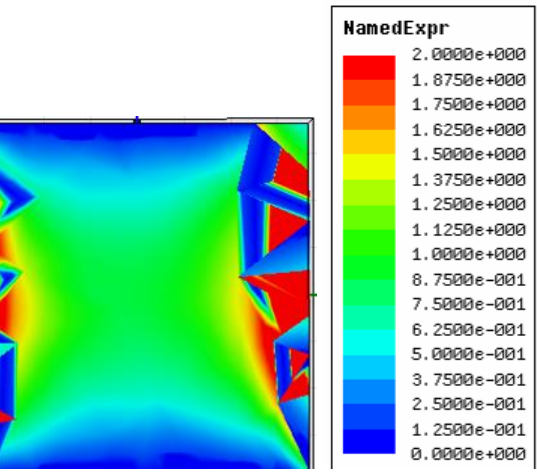 \\
\hline Fase & 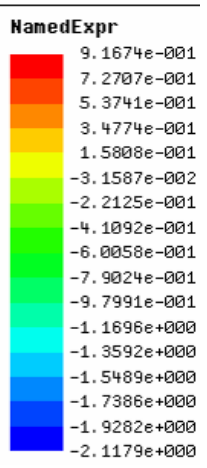 & & & 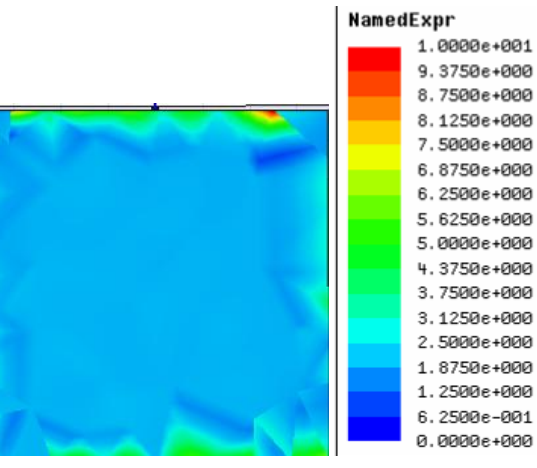 \\
\hline
\end{tabular}




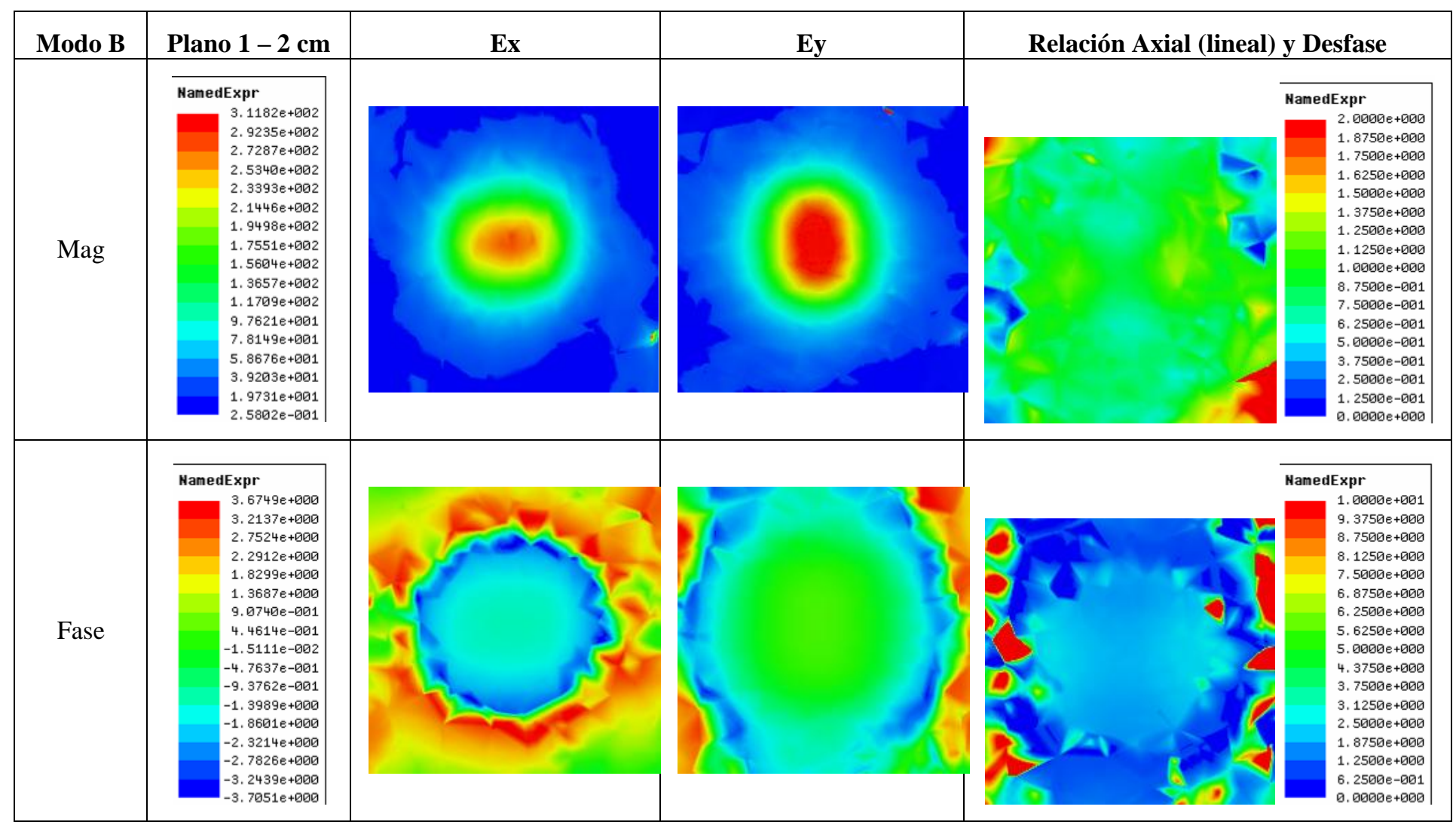




\begin{tabular}{|c|c|c|c|c|}
\hline Modo B & Plano 2 & $\mathbf{E x}$ & Ey & Relación Axial (lineal) y Desfase \\
\hline Mag & 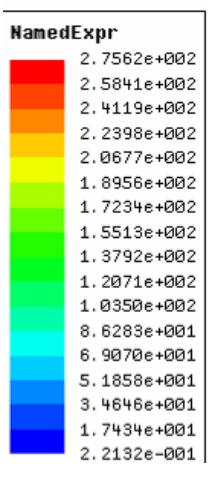 & & & 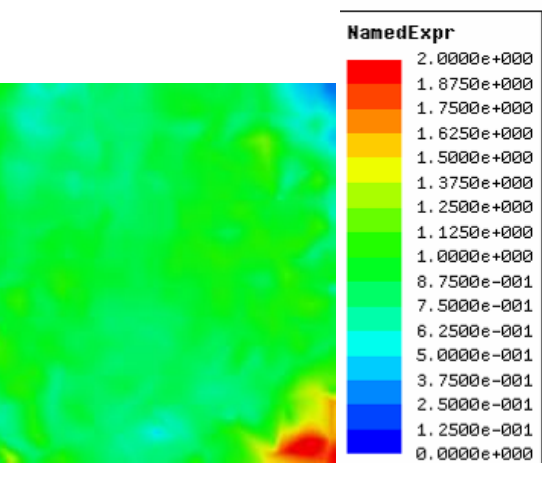 \\
\hline Fase & 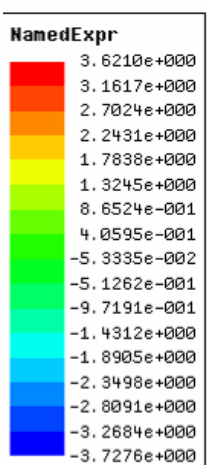 & & & 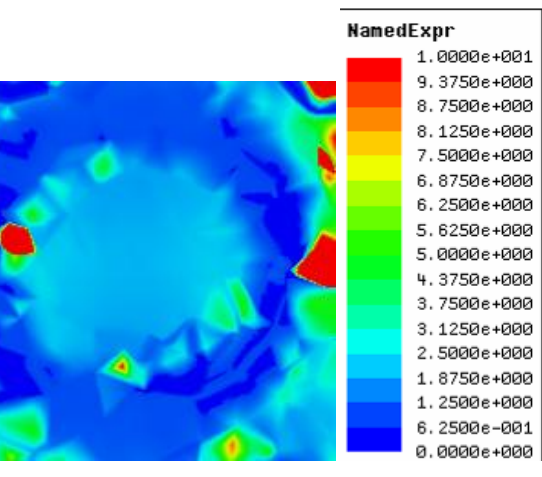 \\
\hline
\end{tabular}




\begin{tabular}{|c|c|c|c|c|}
\hline Modo B & Plano 3 & $\mathbf{E x}$ & Ey & Relación Axial (lineal) y Desfase \\
\hline Mag & 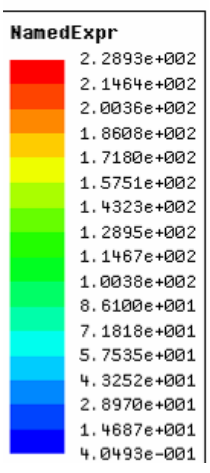 & & & 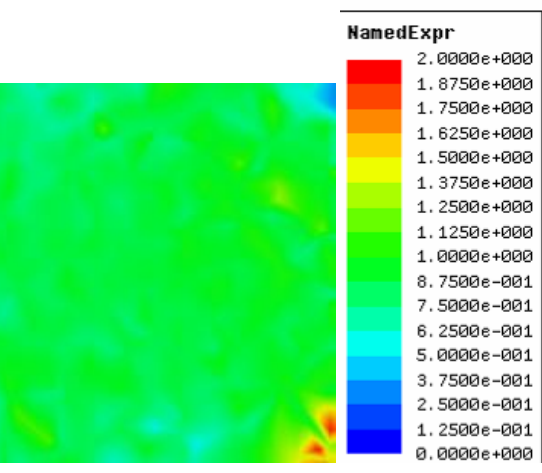 \\
\hline Fase & 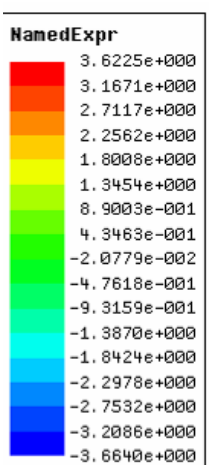 & & & 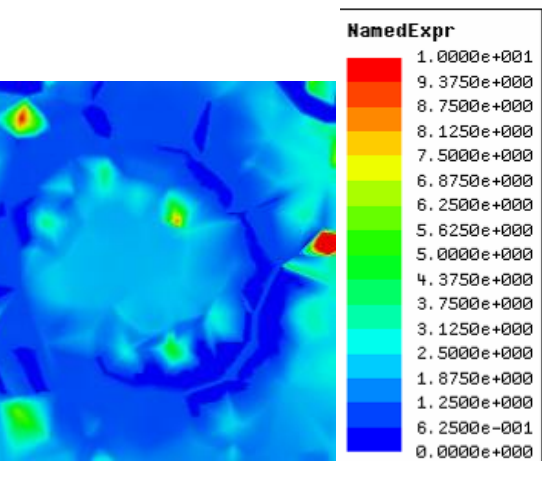 \\
\hline
\end{tabular}




\begin{tabular}{|c|c|c|c|c|}
\hline Modo B & Plano 4 & $\mathbf{E x}$ & Ey & Relación Axial (lineal) y Desfase \\
\hline Mag & 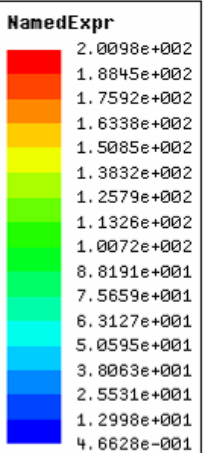 & & & $\begin{array}{l}\text { HamedExpr } \\
\begin{array}{|c|c}2.0000 e+\square 00 \\
1.8750 e+000 \\
1.7500 e+000 \\
1.6250 e+\square 00 \\
1.5000 e+000 \\
1.3750 e+000 \\
1.2500 e+000 \\
1.1250 e+\square 00 \\
1.0000 e+000 \\
8.7500 e-001 \\
7.5000 e-001 \\
6.2500 e-001 \\
5.0000 e-001 \\
3.7500 e-001 \\
2.5000 e-001 \\
1.2500 e-001 \\
0.0000 e+000\end{array}\end{array}$ \\
\hline Fase & 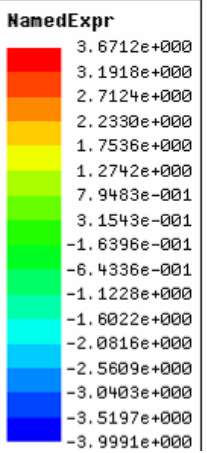 & & & 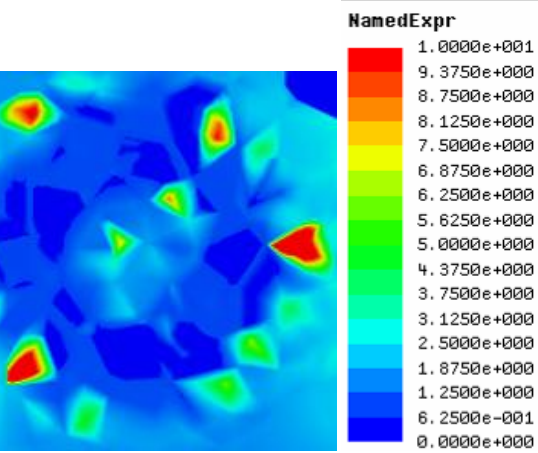 \\
\hline
\end{tabular}




\begin{tabular}{|c|c|c|c|c|}
\hline Modo B & Plano 5 & Ex & Ey & Relación Axial (lineal) y Desfase \\
\hline Mag & 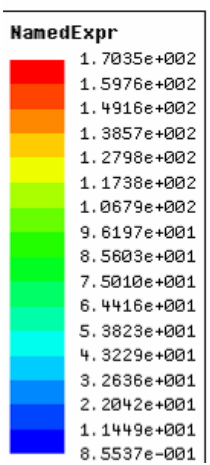 & & & 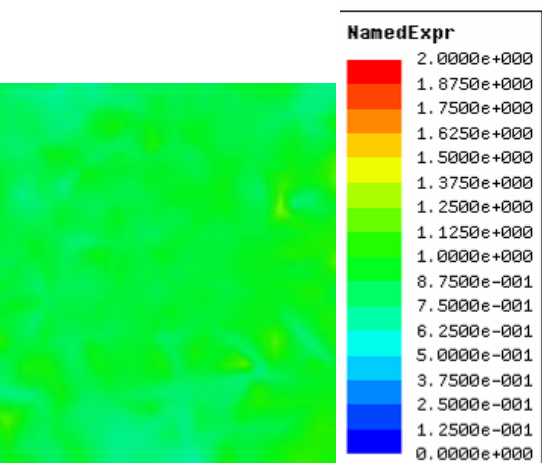 \\
\hline Fase & 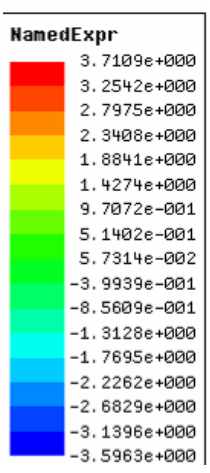 & & & 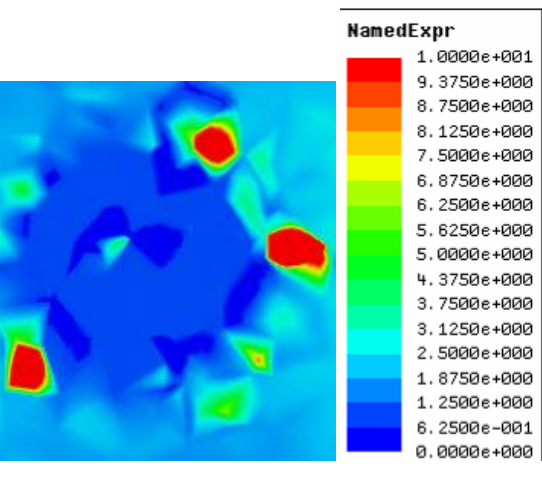 \\
\hline
\end{tabular}




\section{APÉNDICE V. COMPROBACIÓN APLICADOR}

\section{i. Polarización}

En este apartado se muestran los valores de los parámetros de scattering medidos en el experimento de polarización a 2,45 GHz.

Tabla 37. Parámetros scattering - 1 puerto

\begin{tabular}{|c|c|c|c|c|c|c|c|c|c|c|}
\hline & \multicolumn{2}{|c|}{ S11 } & \multicolumn{2}{|c|}{ S21 } & \multicolumn{2}{|c|}{ S12 } & \multicolumn{2}{|c|}{ S22 } \\
\hline & & & $\begin{array}{c}\text { Mag } \\
\text { dB }\end{array}$ & fase $^{\circ}$ & $\begin{array}{c}\text { Mag } \\
\text { dB }\end{array}$ & fase $^{o}$ & $\begin{array}{c}\text { Mag } \\
\text { dB }\end{array}$ & fase $^{o}$ & $\begin{array}{c}\text { Mag } \\
\text { dB }\end{array}$ & fase $^{o}$ \\
\hline \multirow{6}{*}{ V } & $2 \mathrm{~m}$ & Aire & $-13,84$ & 195,99 & $-34,08$ & 280,98 & $-33,54$ & 283,07 & $-14,27$ & 322,38 \\
\hline & \multirow{3}{*}{$6,5 \mathrm{~cm}$} & Aire & $-11,93$ & 223,72 & $-9,95$ & 81,77 & $-9,35$ & 83,03 & $-10,73$ & 308,67 \\
\hline & & $\begin{array}{c}\text { Madera } \\
\text { Tangencial }\end{array}$ & $-9,33$ & 198,28 & $-11,41$ & 45,37 & $-10,59$ & 46,28 & $-11,40$ & 296,07 \\
\hline & & $\begin{array}{c}\text { Madera } \\
\text { Radial } \\
\end{array}$ & $-9,22$ & 199,19 & $-11,52$ & 45,56 & $-10,81$ & 46,32 & $-11,20$ & 296,26 \\
\hline & \multirow[b]{2}{*}{$14 \mathrm{~cm}$} & Aire & $-12,20$ & 201,98 & $-13,46$ & 234,81 & $-12,75$ & 236,41 & $-10,58$ & 308,65 \\
\hline & & $\begin{array}{c}\text { Madera } \\
\text { Viga }\end{array}$ & $-14,30$ & 187,82 & $-17,74$ & 86,79 & $-16,99$ & 88,64 & $-9,86$ & 318,22 \\
\hline \multirow{6}{*}{$\mathrm{H}$} & $2 \mathrm{~m}$ & Aire & $-13,89$ & 195,75 & $-32,71$ & 73,53 & $-32,17$ & 74,44 & $-14,41$ & 320,58 \\
\hline & \multirow{3}{*}{$6,5 \mathrm{~cm}$} & Aire & $-9,78$ & 179,18 & $-9,23$ & 187,48 & $-8,52$ & 189,60 & $-12,82$ & 334,66 \\
\hline & & $\begin{array}{c}\text { Madera } \\
\text { Tangencial }\end{array}$ & $-19,91$ & 183,10 & $-12,27$ & 143,64 & $-11,56$ & 144,01 & $-8,63$ & 311,98 \\
\hline & & $\begin{array}{c}\text { Madera } \\
\text { Radial } \\
\end{array}$ & $-18,12$ & 195,89 & $-12,38$ & 144,03 & $-11,62$ & 144,15 & $-8,79$ & 313,89 \\
\hline & \multirow[b]{2}{*}{$14 \mathrm{~cm}$} & Aire & $-15,61$ & 184,12 & $-12,27$ & 312,01 & $-11,55$ & 311,42 & $-9,23$ & 307,03 \\
\hline & & $\begin{array}{c}\text { Madera } \\
\text { Viga }\end{array}$ & $-14,94$ & 180,62 & $-18,99$ & 115,19 & $-18,39$ & 114,18 & $-10,25$ & 291,75 \\
\hline
\end{tabular}

Tabla 38. Parámetros scattering - 2 puertos

\begin{tabular}{|c|c|c|c|c|c|c|c|c|c|c|}
\hline & \multicolumn{2}{|c|}{ S11 } & \multicolumn{2}{|c|}{ S21 } & \multicolumn{2}{|c|}{ S12 } & \multicolumn{2}{|c|}{ S22 } \\
\hline & & & $\begin{array}{c}\text { Mag } \\
\text { dB }\end{array}$ & fase $^{o}$ & $\begin{array}{c}\text { Mag } \\
\text { dB }\end{array}$ & fase $^{\circ}$ & $\begin{array}{c}\text { Mag } \\
\text { dB }\end{array}$ & fase $^{\circ}$ & $\begin{array}{c}\text { Mag } \\
\text { dB }\end{array}$ & fase $^{o}$ \\
\hline \multirow{4}{*}{ V } & $2 \mathrm{~m}$ & Aire & $-19,88$ & 108,13 & $-32,15$ & 278,16 & $-32,06$ & 279,02 & $-14,28$ & 346,78 \\
\hline & \multirow{3}{*}{$6,5 \mathrm{~cm}$} & Aire & $-26,27$ & 41,26 & $-8,56$ & 89,98 & $-8,09$ & 91,31 & $-12,40$ & 350,01 \\
\hline & & $\begin{array}{c}\text { Madera } \\
\text { Tangencial }\end{array}$ & $-13,03$ & 199,47 & $-12,09$ & 37,63 & $-11,09$ & 40,85 & $-9,12$ & 327,70 \\
\hline & & $\begin{array}{c}\text { Madera } \\
\text { Radial }\end{array}$ & $-12,94$ & 197,45 & $-12,31$ & 37,75 & $-11,29$ & 41,04 & $-8,89$ & 328,12 \\
\hline
\end{tabular}


APÉNDICE V

\begin{tabular}{|c|c|c|c|c|c|c|c|c|c|c|}
\hline \multirow{2}{*}{$14 \mathrm{~cm}$} & Aire & $-24,26$ & 97,91 & $-12,46$ & 245,57 & $-11,70$ & 246,75 & $-9,52$ & 341,91 \\
\cline { 3 - 10 } & $\begin{array}{c}\text { Madera } \\
\text { Viga }\end{array}$ & $-18,23$ & 212,71 & $-19,62$ & 49,95 & $-18,95$ & 51,46 & $-9,65$ & 324,53 \\
\hline \multirow{3}{*}{$\mathrm{H}$} & Aire & $-19,88$ & 107,84 & $-37,49$ & 272,45 & $-37,47$ & 276,30 & $-14,50$ & 344,85 \\
\hline \multirow{3}{*}{$6,5 \mathrm{~cm}$} & Aire & $-21,89$ & 79,53 & $-14,96$ & 21,80 & $-14,51$ & 20,42 & $-15,76$ & 310,41 \\
\cline { 3 - 10 } & $\begin{array}{c}\text { Madera } \\
\text { Tangencial }\end{array}$ & $-13,25$ & 207,57 & $-14,75$ & 341,57 & $-14,66$ & 344,58 & $-21,01$ & 45,54 \\
\cline { 3 - 10 } & $\begin{array}{c}\text { Madera } \\
\text { Radial }\end{array}$ & $-13,04$ & 196,38 & $-14,65$ & 337,91 & $-14,50$ & 340,38 & $-21,51$ & 39,17 \\
\cline { 2 - 10 } & \multirow{3}{*}{$14 \mathrm{~cm}$} & Aire & $-21,92$ & 109,59 & $-16,92$ & 194,83 & $-16,45$ & 195,47 & $-12,89$ & 320,59 \\
\cline { 2 - 9 } & $\begin{array}{c}\text { Madera } \\
\text { Viga }\end{array}$ & $-18,69$ & 210,74 & $-21,90$ & 48,79 & $-21,26$ & 47,83 & $-10,90$ & 322,51 \\
\hline
\end{tabular}

\section{ii. Campo en la superficie irradiada}

Las medidas de Intensidad de campo a 2,5m desde el centro del aplicador y radiando en dirección al piso son mostradas en las siguientes tablas.

Tabla 39. Intensidad de Campo Eléctrico $(\mathrm{V} / \mathrm{m})-1 \mathrm{~kW}$

\begin{tabular}{|c|c|c|c|c|c|c|c|c|c|c|c|c|}
\hline & $0^{\circ}$ & & & & & & $8^{\circ}$ & $210^{\circ}$ & $240^{\circ}$ & $270^{\circ}$ & $300^{\circ}$ & 3 \\
\hline \multicolumn{13}{|c|}{ Itura $0 \mathrm{~cm}$} \\
\hline 1 & 14,2 & 37,0 & 28,0 & 6,3 & 18,0 & 18,9 & 9,1 & 29,3 & 17,6 & 11,5 & 14,5 & 29,7 \\
\hline 2 & & & & 7,5 & 20,6 & 25,4 & & 30,4 & 18,9 & 19,8 & 13,9 & 21 \\
\hline 3 & & & & 7,6 & 20,3 & & & 30,5 & 17,8 & 20,1 & 13,5 & 20,5 \\
\hline 4 & & & & & & & & 30,5 & & 0,0 & & 20,0 \\
\hline 5 & 15,8 & 32,9 & & 7,7 & & & & 30,1 & 17,8 & 20,1 & 13,2 & 20,1 \\
\hline 6 & 15,9 & 33,0 & 24,1 & 7,7 & 19,9 & 24,9 & 9,5 & 30,4 & 17,5 & 20,1 & & 19,8 \\
\hline 7 & 6,1 & 2,1 & & 7,8 & 9,8 & 24,9 & & 30,2 & 17,5 & 20,2 & 3,0 & 19,5 \\
\hline 8 & 162 & 320 & & & 19,7 & 24,9 & & 30,5 & 17,5 & 20,3 & 12,8 & 19,5 \\
\hline 9 & 16,3 & 32,8 & & 7,8 & 9,9 & & 9,6 & 30,5 & 7,2 & 0,3 & 12,6 & 19,2 \\
\hline 10 & 16,4 & 2,9 & 23,6 & 7,8 & 19,3 & 24,8 & & 30,2 & 17,4 & 20,4 & 12,6 & 19,1 \\
\hline 11 & 16,6 & 32,6 & 23,6 & 7,8 & 19,7 & 24,5 & 9,6 & 30,3 & 17,2 & 20,3 & 12,3 & 19,1 \\
\hline 12 & 16,7 & 2,8 & & 7,9 & 19,4 & 24,5 & & 30,1 & 17,0 & 20,4 & 12,5 & 19,0 \\
\hline 13 & 160 & & & & & 24,5 & & & 17,0 & 20,5 & & 18 \\
\hline 14 & 16,9 & 32,5 & 23,1 & 7,9 & 18,8 & 24,5 & 9,7 & 30,1 & 17,1 & 20,5 & 12,2 & 18,8 \\
\hline 15 & 17,0 & 32,8 & & & & & & & 16,9 & 20,5 & 12,2 & 18, \\
\hline 16 & 17,1 & 33,0 & 23,3 & 7,8 & 18,8 & 24,4 & 9,8 & 29,9 & 16,9 & 20,6 & 12,2 & 18,6 \\
\hline 17 & 1 & 2,7 & & & & & & & 16,7 & 20 & & 18 , \\
\hline 18 & 17,3 & 32,9 & 23,2 & 7,9 & 18,9 & 24,5 & & 29,9 & 16,7 & 20,6 & 11,8 & 18,6 \\
\hline 19 & 17,3 & 32,6 & & & 18,7 & 24,4 & 9,8 & 30,1 & 16,5 & 20,6 & 11,7 & 18, \\
\hline
\end{tabular}




\begin{tabular}{|c|c|c|c|c|c|c|c|c|c|c|c|c|}
\hline \multicolumn{13}{|c|}{ Altura $100 \mathrm{~cm}$} \\
\hline 1 & 15,6 & 34,8 & 12,1 & 17,5 & 24,8 & 17,7 & 28,1 & 26,3 & 11,8 & 20,4 & 15,1 & 3,3 \\
\hline 2 & 15,8 & 34,3 & 14,4 & 8,8 & 23,9 & 23,6 & 27,9 & 28,3 & 3,1 & 14,3 & 14,5 & 6,8 \\
\hline 3 & 15,8 & 33,9 & 14,3 & 8,3 & 22,5 & 23,8 & 27,4 & 28,4 & 3,0 & 10,3 & 14,4 & 7,1 \\
\hline 4 & 15,6 & 33,7 & 14,4 & 7,7 & 22,2 & 24,0 & 27,4 & 28,6 & 2,9 & 10,4 & 14,2 & 7,3 \\
\hline 5 & 15,6 & 33,3 & 14,4 & 7,5 & 22,0 & 24,1 & 27,2 & 28,5 & 2,8 & 10,4 & 13,9 & 7,4 \\
\hline 6 & 15,4 & 33,5 & 14,3 & 7,4 & 21,9 & 24,2 & 27,4 & 28,7 & 2,7 & 10,4 & 14,0 & 7,5 \\
\hline 7 & 15,3 & 33,5 & 14,4 & 7,3 & 21,7 & 24,3 & 27,4 & 28,7 & 2,7 & 10,5 & 13,9 & 7,6 \\
\hline 8 & 15,1 & 33,5 & 14,4 & 6,9 & 21,2 & 24,5 & 27,3 & 28,7 & 2,7 & 10,5 & 13,9 & 7,6 \\
\hline 9 & 15,2 & 33,3 & 14,4 & 6,8 & 21,1 & 24,6 & 27,1 & 28,7 & 2,7 & 10,6 & 14,0 & 7,7 \\
\hline 10 & 15,3 & 33,1 & 14,3 & 6,5 & 21,0 & 24,6 & 27,1 & 28,9 & 2,7 & 10,6 & 13,7 & 7,7 \\
\hline 11 & 15,2 & 32,9 & 14,4 & 6,3 & 21,0 & 24,7 & 27,0 & 28,9 & 2,6 & 10,7 & 13,8 & 7,8 \\
\hline 12 & 15,0 & 32,9 & 14,5 & 6,2 & 20,7 & 24,7 & 27,0 & 28,9 & 2,5 & 10,7 & 13,7 & 7,9 \\
\hline 13 & 15,1 & 32,9 & 14,4 & 6,0 & 20,7 & 24,8 & 27,0 & 28,8 & 2,6 & 10,7 & 13,7 & 7,9 \\
\hline 14 & 15,0 & 33,0 & 14,4 & 6,1 & 20,5 & 24,9 & 26,8 & 28,7 & 2,5 & 10,8 & 13,5 & 8,0 \\
\hline 15 & 14,8 & 33,1 & 14,4 & 6,0 & 20,3 & 24,8 & 26,8 & 28,8 & 2,5 & 10,8 & 13,5 & 8,0 \\
\hline 16 & 14,8 & 32,8 & 14,5 & 6,0 & 20,1 & 24,9 & 26,8 & 28,9 & 2,4 & 10,8 & 13,5 & 8,1 \\
\hline 17 & 14,9 & 32,9 & 14,4 & 5,8 & 20,2 & 25,0 & 26,7 & 28,9 & 2,4 & 10,8 & 13,5 & 8,1 \\
\hline 18 & 14,9 & 33,0 & 14,4 & 5,8 & 20,3 & 24,9 & 26,8 & 29,0 & 2,4 & 10,9 & 13,5 & 8,2 \\
\hline 19 & 14,7 & 32,8 & 14,4 & 5,5 & 20,1 & 25,0 & 26,8 & 28,9 & 2,4 & 10,9 & 13,5 & 8,2 \\
\hline \multicolumn{13}{|c|}{ Altura $150 \mathrm{~cm}$} \\
\hline 1 & 17,4 & 11,2 & 24,1 & 18,0 & 13,1 & 22,4 & 21,9 & 13,2 & 16,2 & 11,1 & 29,0 & 22,9 \\
\hline 2 & 10,4 & 8,6 & 7,9 & 13,6 & 12,9 & 5,6 & 19,5 & 11,3 & 15,4 & 10,0 & 27,0 & 31,3 \\
\hline 3 & 10,4 & 7,0 & 7,5 & 10,3 & 12,9 & 5,7 & 15,3 & 11,4 & 15,1 & 10,0 & 27,1 & 31,7 \\
\hline 4 & 10,4 & 7,0 & 7,4 & 10,3 & 12,9 & 5,7 & 15,5 & 11,2 & 15,2 & 10,1 & 27,1 & 30,9 \\
\hline 5 & 10,3 & 6,9 & 7,4 & 10,4 & 12,8 & 5,7 & 15,5 & 11,1 & 15,2 & 10,1 & 27,3 & 30,8 \\
\hline 6 & 10,2 & 6,8 & 7,1 & 10,3 & 12,8 & 5,6 & 15,6 & 11,1 & 15,2 & 10,2 & 27,4 & 31,0 \\
\hline 7 & 10,3 & 6,7 & 7,0 & 10,4 & 12,7 & 5,6 & 15,5 & 10,9 & 15,3 & 10,2 & 27,4 & 30,3 \\
\hline 8 & 10,1 & 6,4 & 6,7 & 10,3 & 12,7 & 5,6 & 15,3 & 10,9 & 15,3 & 10,3 & 27,4 & 30,7 \\
\hline 9 & 10,0 & 6,6 & 6,5 & 10,4 & 12,7 & 5,6 & 15,2 & 10,8 & 15,2 & 10,3 & 27,4 & 30,3 \\
\hline 10 & 10,0 & 6,4 & 6,5 & 10,4 & 12,6 & 5,6 & 15,3 & 10,8 & 15,3 & 10,3 & 27,4 & 30,1 \\
\hline 11 & 9,9 & 6,4 & 6,2 & 10,4 & 12,5 & 5,6 & 15,3 & 10,6 & 15,3 & 10,4 & 27,4 & 30,6 \\
\hline 12 & 9,9 & 6,3 & 6,4 & 10,3 & 12,6 & 5,6 & 15,2 & 10,7 & 15,4 & 10,4 & 27,4 & 30,4 \\
\hline 13 & 9,8 & 6,4 & 6,2 & 10,4 & 12,5 & 5,5 & 15,4 & 10,7 & 15,4 & 10,5 & 27,5 & 30,2 \\
\hline 14 & 9,9 & 6,3 & 5,9 & 10,4 & 12,5 & 5,5 & 15,2 & 10,7 & 15,4 & 10,5 & 27,5 & 30,0 \\
\hline 15 & 9,6 & 6,3 & 5,9 & 10,4 & 12,5 & 5,5 & 15,2 & 10,7 & 15,3 & 10,6 & 27,5 & 29,9 \\
\hline
\end{tabular}




\begin{tabular}{|l|l|l|l|l|l|l|l|l|l|l|l|l|}
16 & 9,5 & 6,2 & 5,9 & 10,4 & 12,5 & 5,5 & 15,2 & 10,5 & 15,4 & 10,6 & 27,5 & 30,2 \\
\hline 17 & 9,5 & 6,1 & 5,8 & 10,4 & 12,6 & 5,5 & 15,0 & 10,5 & 15,4 & 10,6 & 27,6 & 30,2 \\
\hline
\end{tabular}

\section{Altura 175 cm}

\begin{tabular}{|r|r|r|r|r|r|r|r|r|r|r|r|r|}
\hline 1 & 46,8 & 34,9 & 27,7 & 18,3 & 26,1 & 20,3 & 2,3 & 10,8 & 14,9 & 20,6 & 18,2 & 3,4 \\
\hline 2 & 23,5 & 28,4 & 24,0 & 14,9 & 21,1 & 18,5 & 10,9 & 8,9 & 14,4 & 16,8 & 17,2 & 3,2 \\
\hline 3 & 23,6 & 27,4 & 23,7 & 14,6 & 21,3 & 18,3 & 11,0 & 8,1 & 14,2 & 16,5 & 16,5 & 2,6 \\
\hline 4 & 23,3 & 27,1 & 23,4 & 14,5 & 21,5 & 18,0 & 11,1 & 8,0 & 14,2 & 16,4 & 16,4 & 2,3 \\
\hline 5 & 23,3 & 27,8 & 23,3 & 14,5 & 21,6 & 17,8 & 11,1 & 7,9 & 14,1 & 16,1 & 16,0 & 2,2 \\
\hline 6 & 23,2 & 28,2 & 23,4 & 14,3 & 21,2 & 17,8 & 11,2 & 7,8 & 14,1 & 16,0 & 16,0 & 2,1 \\
\hline 7 & 23,2 & 28,2 & 23,0 & 14,2 & 21,3 & 17,7 & 11,2 & 7,7 & 14,1 & 15,8 & 15,5 & 2,0 \\
\hline 8 & 22,8 & 28,5 & 23,1 & 14,2 & 21,4 & 17,5 & 11,2 & 7,7 & 14,0 & 15,9 & 15,6 & 2,0 \\
\hline 9 & 22,9 & 28,1 & 22,7 & 14,2 & 21,4 & 17,5 & 11,2 & 7,7 & 13,8 & 15,5 & 15,3 & 2,0 \\
\hline 10 & 23,0 & 29,2 & 22,7 & 14,0 & 21,3 & 17,4 & 11,2 & 7,6 & 13,8 & 15,8 & 15,4 & 1,9 \\
\hline 11 & 22,9 & 28,5 & 22,6 & 14,1 & 21,5 & 17,4 & 11,3 & 7,5 & 13,7 & 15,4 & 15,2 & 1,9 \\
\hline 12 & 23,0 & 28,6 & 22,5 & 14,1 & 21,2 & 17,4 & 11,3 & 7,4 & 13,6 & 15,6 & 15,0 & 1,9 \\
\hline 13 & 23,0 & 28,9 & 22,4 & 13,9 & 21,1 & 17,3 & 11,3 & 7,4 & 13,9 & 15,5 & 14,8 & 1,8 \\
\hline 14 & 22,7 & 28,6 & 22,6 & 14,0 & 21,0 & 17,3 & 11,3 & 7,3 & 13,7 & 15,4 & 14,8 & 1,8 \\
\hline 15 & 22,6 & 28,5 & 22,3 & 13,9 & 20,8 & 17,4 & 11,3 & 7,3 & 13,6 & 15,3 & 14,7 & 1,8 \\
\hline 16 & 22,5 & 28,3 & 22,3 & 13,9 & 20,6 & 17,3 & 11,3 & 7,2 & 13,6 & 15,3 & 14,7 & 1,8 \\
\hline 17 & 22,4 & 28,4 & 22,3 & 13,7 & 20,4 & 17,2 & 11,3 & 7,2 & 13,5 & 15,2 & 14,6 & 1,8 \\
\hline 18 & 22,2 & 28,6 & 22,0 & 13,7 & 20,1 & 17,2 & 11,2 & 7,2 & 13,6 & 15,1 & 14,7 & 1,8 \\
\hline
\end{tabular}

Tabla 40. Intensidad de Campo Eléctrico (V/m)- $2 \mathrm{~kW}$

\begin{tabular}{|c|c|c|c|c|c|c|c|c|c|c|c|c|}
\hline & $\mathbf{0}^{\mathbf{o}}$ & & $60^{\circ}$ & $90^{\circ}$ & $120^{\circ}$ & $150^{\circ}$ & $180^{\circ}$ & $210^{\circ}$ & $240^{\circ}$ & $270^{\circ}$ & $300^{\circ}$ & $330^{\circ}$ \\
\hline \multicolumn{13}{|c|}{ Altura $0 \mathrm{~cm}$} \\
\hline 1 & 18,3 & 529 & 59,0 & 30,3 & 39,0 & 34,5 & 42,2 & 47,5 & 42,1 & 36,2 & 43,6 & 51,5 \\
\hline 2 & & & & & 37,5 & 34,4 & & 46,7 & & 34,6 & 44,3 & 50,5 \\
\hline 3 & 17,9 & & 572 & 204 & 36,7 & & & 46,9 & 37,8 & 34,2 & 43,8 & 50,0 \\
\hline 4 & 18,3 & 0,7 & 50,1 & $3 \mathrm{~J}, 4$ & 35,9 & 32,4 & 44,3 & 46,5 & 31,9 & 33,9 & 43,7 & 49,3 \\
\hline 5 & 18,2 & 49,0 & 56,6 & 32,8 & 35,9 & 33,2 & 44,1 & 46,6 & 37,6 & 34,5 & 43,6 & 49,5 \\
\hline 6 & 181 & & 558 & & & & & 46,6 & & & & 49,0 \\
\hline 7 & 18,1 & 7,8 & 55,4 & & 35,9 & 33,8 & 45,7 & 46,4 & 37,6 & 4,6 & 43,2 & 48,9 \\
\hline 8 & 18,0 & 47,5 & 540 & 30, & 35,9 & & 45,2 & 46,4 & & & 43,4 & 48,7 \\
\hline 9 & 18,0 & 47,3 & 54,8 & & & & & & & & 43,6 & 48,6 \\
\hline \multicolumn{13}{|c|}{ Altura $100 \mathrm{~cm}$} \\
\hline 1 & 44,8 & 56,7 & 35,7 & 38,6 & 58,8 & & 81,7 & 86,2 & 44,0 & 63,1 & 58,9 & 30,5 \\
\hline 2 & 44,3 & 55,0 & 33,5 & 38,1 & 56,3 & 44,6 & 69,5 & 81,1 & 43,0 & 61,4 & 58,9 & 30,2 \\
\hline
\end{tabular}




\begin{tabular}{|c|c|c|c|c|c|c|c|c|c|c|c|c|}
\hline & 38,0 & 55,3 & 2,7 & 37,4 & 56,0 & 40,5 & 66,2 & 80,4 & 42,3 & 61,1 & 58,6 & 30 , \\
\hline & 37,6 & 55,6 & 30,9 & 370 & 54,6 & 40,5 & 65,3 & 80,4 & 42,1 & 61,1 & 58,6 & 200 \\
\hline & 7,6 & & & & & & & & & & & \\
\hline & 7,2 & $v_{1}$ & 0,1 & & & 39,8 & 64,8 & 78,9 & & 0,5 & 58,4 & 29,6 \\
\hline & 36,8 & & 9,7 & & 52,2 & 39,4 & 64,2 & 78,9 & & 60,4 & 58,4 & 29,4 \\
\hline & 36,7 & & 9,0 & & 2 & & & 78,2 & & 0,6 & 3,3 & 29,3 \\
\hline & 36,4 & 5 & 8,7 & 36,8 & 49,7 & 36,0 & 35,8 & 78,3 & 1,2 & 0,2 & 8,3 & 29,2 \\
\hline \multicolumn{13}{|c|}{ Altura $150 \mathrm{~cm}$} \\
\hline 1 & 34,4 & 44,6 & 31,6 & 39,3 & 21,2 & 25,5 & 30,3 & 37,0 & & 34,6 & 53,9 & 40,4 \\
\hline 2 & 35,2 & 157 & 32,2 & 37,3 & 21,8 & 267 & 30,3 & 356 & 15,2 & 35,1 & 54,3 & J7, \\
\hline 3 & 35,0 & 45,9 & 2,5 & 37,6 & 21,8 & 26,8 & 30,6 & 36,3 & & 5,6 & 54,8 & 40,0 \\
\hline 4 & 35,3 & 46,1 & 32,7 & 36,7 & 21,5 & 26,8 & 31,0 & 36,4 & 4,9 & 36,0 & 54,7 & 39,1 \\
\hline$J$ & 35,3 & 46,0 & 32,6 & 50,2 & 21,7 & 20,0 & 31,3 & 36,8 & 15,0 & 36,1 & 54,6 & 39,0 \\
\hline 6 & 35,3 & 46 , & 2,8 & 36,3 & 22,2 & 26,6 & 31,2 & 36,2 & & 36,4 & 4,4 & 39,6 \\
\hline 7 & 35,4 & 46,5 & 32,7 & 254 & 226 & 263 & 310 & & & 36,7 & 54,2 & 307 \\
\hline 8 & 5,4 & 46,5 & 3,0 & 0,2 & 22,5 & 0,1 & 30,7 & 36,6 & 4,6 & 6,9 & 4,0 & 40,1 \\
\hline 9 & 35,2 & 46,7 & 32,8 & 36,0 & 22,9 & 26,1 & 24,6 & 38,4 & & 37,1 & 3,8 & 39,9 \\
\hline \multicolumn{13}{|c|}{ Altura $175 \mathrm{~cm}$} \\
\hline 1 & 25,6 & 27,8 & 3,7 & 26,7 & 48,7 & 35,5 & 23,6 & 24,3 & 4,9 & 7,7 & 56,4 & 47,2 \\
\hline$?$ & 23,2 & 26,4 & 23,2 & 27,2 & 46,8 & 31,5 & 23,1 & 24,5 & 55,3 & 38,2 & 55,5 & 41 \\
\hline 3 & 22,8 & 24,9 & 22,9 & 26,7 & 47,6 & & 22,4 & 24,0 & & 8,2 & & 38,6 \\
\hline 4 & 22,9 & 24,8 & 22,5 & 27,7 & 47,6 & 29,8 & 22,3 & 23,7 & 54,2 & 38,4 & 55,6 & 38,4 \\
\hline$J$ & 23,0 & 24,9 & 22,6 & 27,9 & 48,3 & 29,8 & 22,1 & 23,5 & JJ,1 & 38,4 & 55,6 & 38,9 \\
\hline 6 & 23,1 & 25,1 & 2,6 & 27,6 & 47,7 & 30,0 & 22,0 & 23,6 & & 8,6 & 5,8 & 38,7 \\
\hline 7 & 23,0 & 25,3 & 22,6 & 27,7 & 48,8 & 30,0 & 21,8 & 24,1 & 55,5 & 38,9 & 55,9 & 201 \\
\hline 8 & 23,4 & 25,5 & 22,6 & 21,5 & 48,5 & 29,9 & 21,5 & 24,0 & 55,1 & 39,0 & 56,4 & 39,3 \\
\hline 9 & 12,0 & 25,6 & 22,6 & 27,4 & 49,3 & 30,1 & 21,7 & 23,7 & 56,2 & 38,9 & 56,6 & 39,0 \\
\hline
\end{tabular}

Tabla 41. Índices de dispersión Intensidad de campo

\begin{tabular}{|c|r|r|r|r|r|r|r|r|}
\hline \multirow{2}{*}{ Angulo } & \multicolumn{4}{|c|}{$\mathbf{~ k W}$} & \multicolumn{4}{|c|}{$\mathbf{2 ~ k W}$} \\
\cline { 2 - 9 } & $\mathbf{0 ~ m}$ & $\mathbf{1 ~ m}$ & $\mathbf{1 , 5} \mathbf{~ m}$ & $\mathbf{1 , 7} \mathbf{~ m}$ & $\mathbf{0 ~ m}$ & $\mathbf{1 ~ m}$ & $\mathbf{1 , 5} \mathbf{~ m}$ & $\mathbf{1 , 7} \mathbf{~ m}$ \\
\hline \hline $\mathbf{0}^{\mathbf{o}}$ & 0,06 & 0,02 & 0,18 & 0,25 & 0,01 & 0,09 & 0,01 & 0,16 \\
\hline $\mathbf{3 0}^{\mathbf{o}}$ & 0,04 & 0,02 & 0,20 & 0,06 & 0,04 & 0,01 & 0,01 & 0,04 \\
\hline $\mathbf{6 0}^{\mathbf{0}}$ & 0,06 & 0,04 & 0,66 & 0,06 & 0,02 & 0,07 & 0,01 & 0,02 \\
\hline $\mathbf{9 0}^{\circ}$ & 0,05 & 0,41 & 0,19 & 0,07 & 0,05 & 0,02 & 0,03 & 0,02 \\
\hline $\mathbf{1 2 0}^{\mathbf{o}}$ & 0,03 & 0,06 & 0,02 & 0,06 & 0,03 & 0,05 & 0,03 & 0,02 \\
\hline $\mathbf{1 5 0}^{\mathbf{o}}$ & 0,05 & 0,07 & 0,73 & 0,04 & 0,02 & 0,06 & 0,02 & 0,06 \\
\hline
\end{tabular}




\begin{tabular}{|l|l|l|l|l|l|l|l|l|}
$\mathbf{1 8 0}^{\mathbf{}}$ & 0,02 & 0,01 & 0,12 & 0,19 & 0,03 & 0,17 & 0,07 & 0,03 \\
\hline $\mathbf{2 1 0}^{\mathbf{o}}$ & 0,01 & 0,02 & 0,06 & 0,11 & 0,01 & 0,03 & 0,02 & 0,01 \\
\hline $\mathbf{2 4 0}^{\mathbf{o}}$ & 0,03 & 0,80 & 0,01 & 0,03 & 0,04 & 0,02 & 0,06 & 0,01 \\
\hline $\mathbf{2 7 0}^{\circ}$ & 0,10 & 0,22 & 0,03 & 0,08 & 0,02 & 0,01 & 0,02 & 0,01 \\
\hline $\mathbf{3 0 0}^{\mathbf{o}}$ & 0,06 & 0,03 & 0,02 & 0,06 & 0,01 & 0,00 & 0,01 & 0,01 \\
\hline $\mathbf{3 3 0}^{\mathbf{o}}$ & 0,20 & 0,14 & 0,06 & 0,25 & 0,02 & 0,01 & 0,01 & 0,07 \\
\hline
\end{tabular}

\section{iii. Campo en el corte principal del diagrama de radiación}

Las medidas de Intensidad de campo a $2 \mathrm{~m}$ del plano de apertura en dirección de máxima radiación son mostradas en las siguientes tablas.

Tabla 42. Intensidad de Campo (V/m) - sin traje

\begin{tabular}{|c|c|c|c|c|c|c|c|c|}
\hline & $0^{\circ}$ & $45^{\circ}$ & $90^{\circ}$ & $135^{\circ}$ & $180^{\circ}$ & $225^{\circ}$ & $270^{\circ}$ & $315^{\circ}$ \\
\hline \multicolumn{9}{|c|}{ Altura $90 \mathrm{~cm}$} \\
\hline 1 & 2,73 & 146,46 & 50,45 & 24,21 & 24,62 & 23,08 & 34,42 & 176,87 \\
\hline 2 & 210,29 & 146,36 & 48,60 & 26,77 & 22,09 & 22,95 & 34,67 & 178,27 \\
\hline 3 & 312,43 & 146,97 & 47,58 & 28,28 & 22,13 & 22,79 & 34,10 & 179,30 \\
\hline 4 & 314,67 & 147,70 & 47,74 & 28,72 & 21,99 & 22,46 & 34,12 & 179,20 \\
\hline 5 & 316,11 & 147,75 & 48,00 & 28,45 & 22,08 & 22,54 & 34,20 & 179,08 \\
\hline 6 & 312,31 & 147,58 & 47,84 & 28,53 & 21,91 & 22,37 & 34,42 & 179,61 \\
\hline 7 & 314,25 & 147,60 & 48,23 & 28,55 & 21,95 & 22,36 & 34,37 & 179,48 \\
\hline 8 & 314,22 & 147,63 & 47,66 & 28,52 & 21,87 & 22,48 & 34,55 & 179,24 \\
\hline \multicolumn{9}{|c|}{ Altura $140 \mathrm{~cm}$} \\
\hline 1 & 520,13 & 307,35 & 67,28 & 30,62 & 64,76 & 52,64 & 70,12 & 318,4 \\
\hline 2 & 520,13 & 307,61 & 69,73 & 31,36 & 63,48 & 52,74 & 70,32 & 320,62 \\
\hline 3 & 520,13 & 306,19 & 72,20 & 31,97 & 57,43 & 51,25 & 71,61 & 315,61 \\
\hline 4 & 520,13 & 306,84 & 73,25 & 33,31 & 52,92 & 45,97 & 70,99 & 316,8 \\
\hline 5 & 520,13 & 307,85 & 73,67 & 33,14 & 53,25 & 45,22 & 71,13 & 316,03 \\
\hline 6 & 520,13 & 308,02 & 73,79 & 33,21 & 52,70 & 45,06 & 71,41 & 316,9 \\
\hline 7 & 520,13 & 308,15 & 74,18 & 33,11 & 52,66 & 45,15 & 71,57 & 316,61 \\
\hline 8 & 520,13 & 151,52 & 74,31 & 33,12 & 52,32 & 44,98 & 71,65 & 317,08 \\
\hline \multicolumn{9}{|c|}{ Altura $40 \mathrm{~cm}$} \\
\hline 1 & 243,18 & 127,86 & 38,66 & 55,86 & 32,07 & 31,88 & 71,75 & 140,38 \\
\hline 2 & 232,31 & 129,15 & 39,57 & 54,03 & 33,14 & 32,75 & 71,74 & 140,64 \\
\hline 3 & 231,14 & 133,37 & 39,51 & 50,54 & 35,87 & 33,22 & 71,20 & 139,09 \\
\hline 4 & 233,44 & 138,15 & 40,82 & 50,28 & 36,25 & 33,90 & 70,99 & 138,80 \\
\hline 5 & 233,76 & 140,85 & 38,69 & 47,74 & 36,12 & 33,76 & 70,72 & 138,59 \\
\hline
\end{tabular}




\begin{tabular}{|l|l|l|l|l|l|l|l|l|}
\hline 6 & 234,93 & 141,31 & 38,84 & 47,72 & 36,02 & 33,43 & 70,57 & 139,00 \\
\hline 7 & 235,19 & 141,66 & 38,93 & 47,21 & 35,95 & 33,43 & 70,65 & 139,20 \\
\hline 8 & 234,37 & 141,81 & 39,16 & 47,15 & 35,95 & 33,54 & 70,71 & 138,99 \\
\hline
\end{tabular}

Tabla 43. Intensidad de Campo (V/m) - con traje

\begin{tabular}{|c|c|c|c|c|c|c|c|c|}
\hline & $\mathbf{0}^{\mathbf{o}}$ & $45^{\circ}$ & $90^{\circ}$ & $135^{\circ}$ & $180^{\circ}$ & $225^{\circ}$ & $270^{\circ}$ & $315^{\circ}$ \\
\hline \multicolumn{9}{|c|}{ Altura $90 \mathrm{~cm}$} \\
\hline 1 & 41,47 & 66,94 & 21,60 & 14,50 & 13,71 & 8,88 & 21,58 & 56,87 \\
\hline 2 & 161,77 & 64,76 & 19,70 & 13,36 & 13,62 & 8,02 & 20,78 & 50,63 \\
\hline 3 & 139,81 & 62,10 & 19,29 & 12,91 & 11,69 & 7,50 & 20,48 & 46,42 \\
\hline 4 & 134,69 & 56,78 & 18,25 & 11,81 & 11,43 & 6,92 & 18,96 & 46,47 \\
\hline 5 & 128,58 & 54,07 & 17,63 & 11,13 & 11,12 & 6,51 & 19,05 & 46,11 \\
\hline 6 & 119,44 & 53,80 & 17,71 & 11,07 & 10,42 & 6,44 & 19,00 & 46,29 \\
\hline 7 & 108,96 & 53,48 & 17,77 & 11,00 & 10,32 & 6,42 & 18,87 & 46,06 \\
\hline 8 & 109,05 & 53,28 & 17,77 & 10,94 & 10,23 & 6,40 & 18,95 & 46,09 \\
\hline
\end{tabular}

Altura $140 \mathrm{~cm}$

\begin{tabular}{|r|r|r|r|r|r|r|r|r|}
\hline 1 & 123,14 & 59,34 & 16,46 & 5,53 & 9,33 & 7,6 & 15,95 & 85,56 \\
\hline 2 & 121,79 & 58,02 & 15,29 & 6,37 & 8,28 & 7,28 & 14,8 & 86,61 \\
\hline 3 & 121,38 & 56,12 & 13,86 & 6,51 & 7,87 & 6,99 & 14,35 & 87,93 \\
\hline 4 & 124,84 & 52,17 & 13,8 & 6,56 & 7,03 & 6,75 & 13,61 & 86,53 \\
\hline 5 & 119,06 & 47,24 & 13,73 & 6,57 & 7,04 & 6,72 & 12,98 & 86,19 \\
\hline 6 & 115,03 & 47,41 & 13,74 & 6,58 & 7,01 & 6,66 & 13,02 & 85,61 \\
\hline 7 & 109,69 & 47,87 & 13,67 & 6,63 & 7,04 & 6,61 & 12,95 & 84,34 \\
\hline 8 & 83,36 & 47,73 & 13,71 & 6,59 & 7,04 & 6,6 & 12,97 & 81,08 \\
\hline
\end{tabular}

Altura $173 \mathrm{~cm}$

\begin{tabular}{|r|r|r|r|r|r|r|r|r|}
\hline 1 & 80,58 & 96,01 & 39,43 & 17,08 & 18,27 & 17,45 & 51,55 & 111,07 \\
\hline 2 & 153,34 & 94,29 & 38,61 & 18,51 & 18,17 & 17,12 & 49,23 & 100,56 \\
\hline 3 & 148,32 & 91,91 & 36,94 & 18,66 & 18,40 & 16,74 & 45,83 & 92,42 \\
\hline 4 & 142,45 & 87,29 & 34,08 & 18,63 & 18,54 & 16,31 & 45,74 & 92,31 \\
\hline 5 & 144,47 & 87,43 & 33,94 & 18,53 & 18,44 & 16,18 & 45,98 & 91,75 \\
\hline 6 & 134,32 & 86,88 & 33,63 & 18,51 & 18,54 & 16,24 & 45,95 & 92,02 \\
\hline 7 & 133,41 & 86,66 & 33,42 & 18,50 & 18,45 & 16,21 & 45,91 & 91,56 \\
\hline 8 & 133,35 & 86,42 & 33,24 & 18,39 & 18,56 & 16,15 & 45,99 & 91,84 \\
\hline
\end{tabular}


Tabla 44. Intensidad de Campo (V/m) - con traje a 1,5m

\begin{tabular}{|c|c|c|c|c|c|c|c|c|}
\hline & $0^{\mathbf{o}}$ & $45^{\circ}$ & $90^{\circ}$ & $135^{\circ}$ & $180^{\circ}$ & $225^{\circ}$ & $270^{\circ}$ & $315^{\circ}$ \\
\hline \multicolumn{9}{|c|}{ Altura $90 \mathrm{~cm}$} \\
\hline 1 & 88,15 & 67,12 & 28,81 & 16,31 & 16,31 & 12,17 & 27,1 & 60,8 \\
\hline 2 & 176,17 & 66,63 & 27,77 & 16,43 & 17,94 & 11,45 & 25,66 & 59,17 \\
\hline 3 & 170,64 & 66,29 & 25,25 & 16,39 & 18,29 & 11,41 & 24,03 & 57,39 \\
\hline 4 & 171,37 & 63,57 & 25,32 & 16,58 & 18,61 & 10,86 & 24,02 & 56,57 \\
\hline 5 & 170 & 61,2 & 25,32 & 16,48 & 18,49 & 10,44 & 23,9 & 56,48 \\
\hline 6 & 170,09 & 61,46 & 25,3 & 16,55 & 18,38 & 10,41 & 23,91 & 56,58 \\
\hline 7 & 170,4 & 61,44 & 25,23 & 16,52 & 18,34 & 10,29 & 23,65 & 56,7 \\
\hline 8 & 169,4 & 61,44 & 25,19 & 16,49 & 18,25 & 10,24 & 23,88 & 56,94 \\
\hline
\end{tabular}

Tabla 45. Índice de dispersión corte principal

\begin{tabular}{|c|c|c|c|c|c|c|c|c|c|}
\hline \multicolumn{2}{|c|}{ Altura (cm) } & $0^{\circ}$ & $45^{\circ}$ & $90^{\circ}$ & $135^{\circ}$ & $180^{\circ}$ & $225^{\circ}$ & $270^{\circ}$ & $315^{\circ}$ \\
\hline \multirow{3}{*}{ Sin Traje } & 90 & 0,37 & 0,00 & 0,02 & 0,06 & 0,04 & 0,01 & 0,01 & 0,01 \\
\hline & 140 & 0,00 & 0,18 & 0,04 & 0,03 & 0,11 & 0,09 & 0,01 & 0,00 \\
\hline & 40 & 0,01 & 0,04 & 0,02 & 0,07 & 0,05 & 0,02 & 0,01 & 0,01 \\
\hline \multirow{4}{*}{ Con traje } & 90 & 0,27 & 0,11 & 0,08 & 0,12 & 0,12 & 0,14 & 0,06 & 0,09 \\
\hline & 140 & 0,12 & 0,10 & 0,08 & 0,06 & 0,14 & 0,06 & 0,09 & 0,02 \\
\hline & 173 & 0,16 & 0,05 & 0,08 & 0,03 & 0,01 & 0,03 & 0,05 & 0,08 \\
\hline & $90(1,5 \mathrm{~m})$ & 0,17 & 0,04 & 0,06 & 0,01 & 0,04 & 0,07 & 0,05 & 0,03 \\
\hline
\end{tabular}




\section{APÉNDICE VI. TEMPERATURA APLICADOR}

En la Figura 3 se muestra el índice de dispersión de las medidas de temperatura para una aplicación central hechas con el aplicador diseñado y para los tres tipos de muestras tratados.
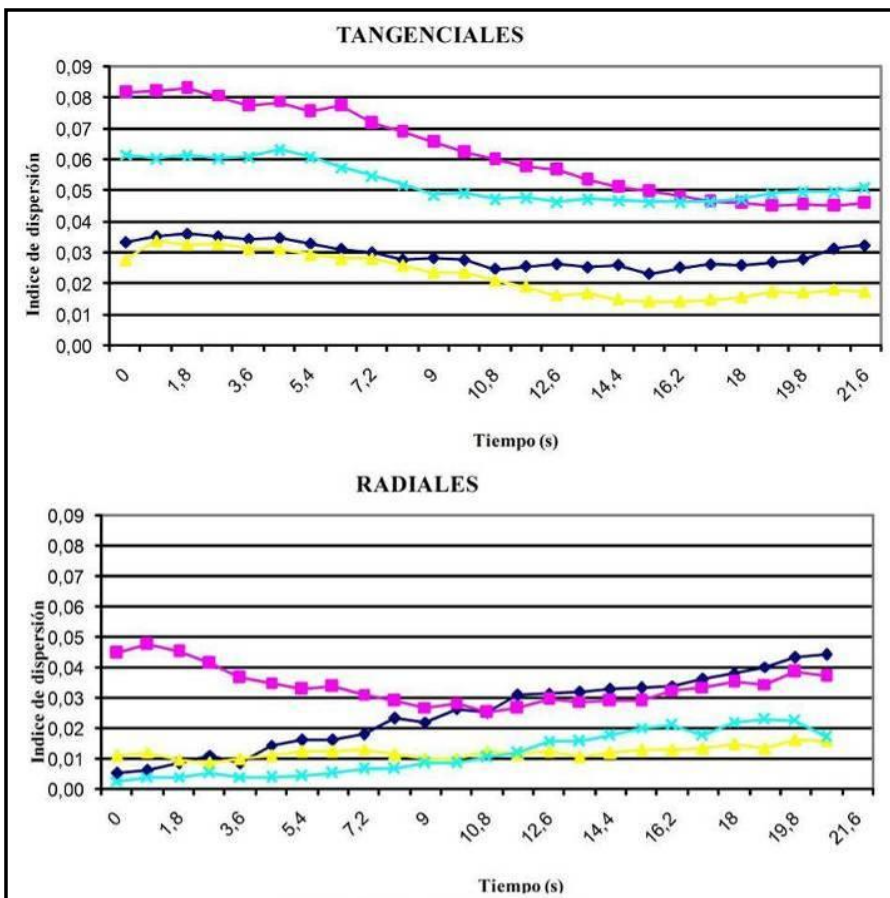

TANGENCIALES JOVENES

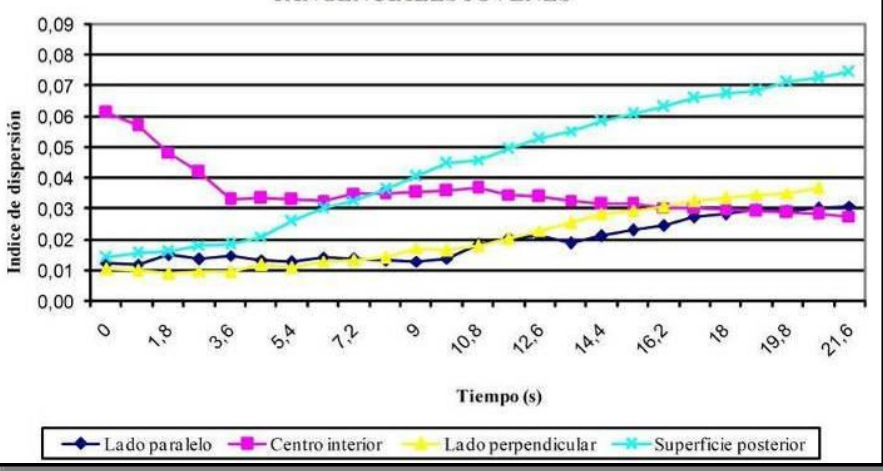

Figura 53. Índice de Dispersión - Aplicación central 
En la Figura 4 se muestra el índice de dispersión de las medidas de temperatura para la aplicación solapada hechas con el aplicador diseñado y para los tres tipos de muestras tratados.

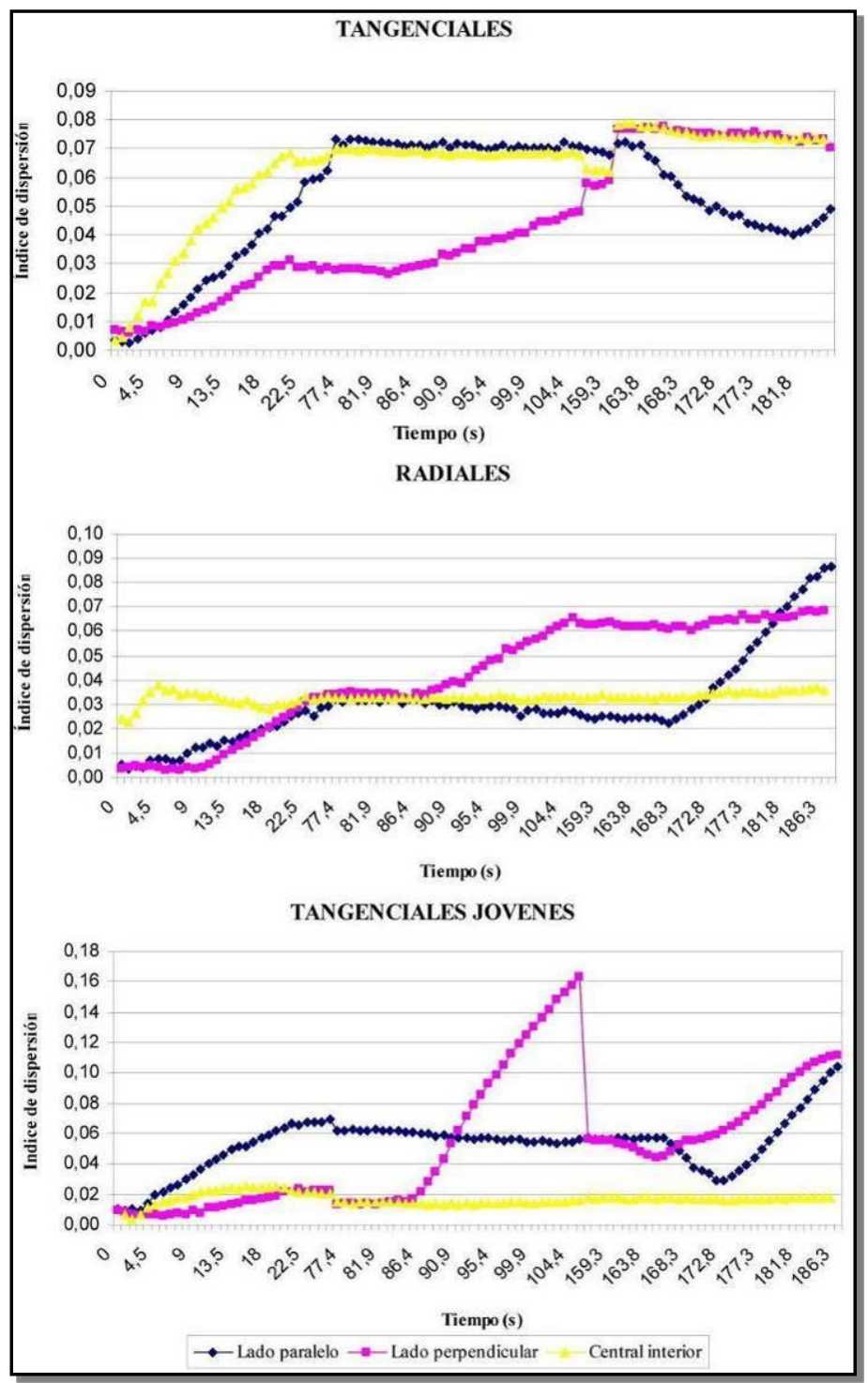

Figura 54. Índice de dispersión - Aplicación solapada 